THE ENIGMA OF QUALITY IN GREEK HIGHER EDUCATION 



\title{
THE ENIGMA OF QUALITY IN GREEK HIGHER EDUCATION
}

\author{
A MIXED METHODS STUDY OF INTRODUCING QUALITY \\ MANAGEMENT INTO GREEK HIGHER EDUCATION
}

\section{DISSERTATION}

\author{
to obtain \\ the degree of doctor at the University of Twente, \\ on the authority of the rector magnificus, \\ prof. dr. H. Brinksma,
}

on account of the decision of the graduation committee,

to be publicly defended

on Friday $4^{\text {th }}$ of March 2011 at 16.45

by

Antigoni Papadimitriou

born on 5 May 1962

in Thessaloniki 
This dissertation has been approved by the promoters:

Prof. dr. J. Enders

Dr. D.F. Westerheijden

ISBN 978-90-365-3155-9

DOI: $10.3990 . / 1.9789036531559$

(c) Antigoni Papadimitriou, 2011

All rights reserved. No part of this publication may be reproduced, stored in a retrieval system of any nature, or transmitted in any form or by any means, electronic, mechanical, now known or hereafter invented, including photocopying or recording, without prior written permission of the author.

Printed by M.I.B. PRINT, the Czech Republic

Published by CHEPSNT, P.O. Box 217, NL-7500 AE Enschede, cheps@mb.utwente.nl

Cover design by WeCre8, Enschede, The Netherlands

Copyrighted tables and figures reproduced with written permission of the owners for both print and electronic re-use.

Figure 2-1, copyright NIST, 2005

Table 2-3, copyright Csizmadia, 2006

Figure 2-2, copyright Trudy Banta, 2006

Figure 3-1, copyright Harrison, 1994, Sage Publication

Figure 4-2, copyright Teddlie \& Tashakkori, 2009

Figure 5-1, copyright DG Research, 2003

Line drawing on back cover, copyright Vera R. Westerheijden, 2010 
To my mother Soultana Kafafian, who taught me that all things are possible with faith and perseverance 
Members of the graduation committee:

Prof. dr. ir. P.C. de Weerd-Nederhof

Prof. dr. ir. O.A.M. Fisscher

Prof. dr. J. Välimaa

Prof. dr. G. Tsiotras 


\section{Preface}

When one writes about organizational change and processes, one discovers that top managers and leaders are often preoccupied with several specific types of changes. They find themselves reacting to new and important external environmental conditions as well as "windows of opportunity", while at the same time, they attempt to monitor and asseses various changes they initiated, and which resulted from their individual beliefs and aspirations.

Many elements combine to forge one's values and belief systems and from my earliest memories, my mother taught me to follow my passion, to seize opportunities in the apparent midst of what others regarded as disasters, and to pursue my dreams. Thus my journey for this book began and without that foundational preparation, I might not have had the tenacity to press forward with the study of organizational change. Additional elements that propelled me into this study were my previous jobs as a university administrator and as a basketball coach. As a basketball coach during summers I travelled and worked in several famous campuses in the US, where I became impressed not only with their athletic facilities or libraries but also by the broader organization and management of those universities. The sudden loss of both my mother and my grandmother in a rather short time led me into thinking about change, which at the time was an emotional crisis that became a "window of opportunity" for me to initiate change in my life's direction. Thus, I decided to travel abroad, to change career objectives, and learn more about management in higher education.

The journey began in New York, were I earned a Masters degree in higher education administration at Baruch College. Because of $9 / 11$ reduced opportunities for scholarships and jobs, I decided to return to Greece, working as teaching research staff at the Department of Economics, while I began a search for a doctoral program in higher education in business schools, because doctorates in higher education studies do not exist in Greece. From those visits, I am grateful to have met one individual, professor Tsiotras, Rector (at that time) of the University of Macedonia, an expert in quality management who offered me the opportunity to begin my doctoral studies on quality management in Greek universities. Since Greece had limited access to books and journals about higher education, I visited several U.S. libraries, and several friends, mostly professors in higher education provided much appreciated help and support. However, professor Tsiotras' appointment as a General Secretary for the Central Macedonia and the difficult 
circumstances on doing research about evaluation and quality management that period (2005) in Greece necessitated my journey to change route and to look for a safe port to complete my PhD.

During my studies in Greece, free on-line publications offered by the Center for Higher Education Studies (CHEPS) at the University of Twente, were a gift from heaven and its work already guided and inspired me, thus, I would never thought of a better place than CHEPS to continue and complete my dissertation.

Greece is well known for hospitality; however, only scholars studying and visiting CHEPS are able to value and appreciate the unique, unforgettable experiences, friendship, and generous hospitality offered by CHEPSonians. In CHEPS' multi-cultural environment I learned that I did not act in a vacuum. Instead, I was surrounded by active researchers whose work interacted with mine and this interaction had an important effect on my thinking and actions. Surroundings like these are especially critical for higher education scholars who can learning by doing, work in a multi-cultural entrepreneurial and visionary environment, learn to listen and to share research products and ideas, mimic the best in the field, contribute to the body of knowledge in this particular area and not only raise professionalization but also cultivate the character of future scholarship in the higher education field.

Now that the journey of writing my $\mathrm{PhD}$ dissertation reaches the final stage, I acknowledge the importance of the many people along the way whom I have been blessed to know, and by those who have inspired me in significant ways. To all of you, I want to thank you for the special contributions that you have made to my experience- a journey that I will never forget, because of all the wonderful people who have helped me to learn and grow.

First and foremost, my sincere gratitude goes to my promoters, Professor Jürgen Enders and Dr. Don F. Westerheijden. I would like to thank Professor Enders' willingness to be the chairperson of my committee, his countless suggestions, time, interest, dedication, and standards of excellence. I would like to thank Professor Enders for his effort to make me to listen. If my mother were alive she would also like to thank him for that. I would like to extend a deep sincere gratitude and very special thanks to Don Westerheijden for being such a wonderful teacher and advisor, for his comprehensive support and constructive guidance during the completion of this study. All these years, his willingness and effort to reply to all of my messages from all over the word, to discuss my ideas in an intelligent and critical way by using all kinds of technologies - Skype, telephon, fax, and regular mail - were the motivation to work even harder for my 
dissertation when the challenges seemed insurmountable. Don's green Skype signal became my Pharos- lighthouse by which I navigated my rough journey. I will never forget that both of my promoters reviewed my work and spent hours not only at CHEPS but during their professional trips worldwide, a lesson of professionalism highly appreciated and I promise to do the same with my future students.

What appears in this book is in reality a tapestry woven to include insights that come from an extended network and I could not have done it without warps and weaves that were instrumental in the completion of my dissertation; people who shared their practices, who commented, and who volunteered their time to be interviewed. Many helping hands and patient hearts are involved in the completion of anyone's dissertation, and this effort is no exception. I would like to thank Dr. Madeline Wing Adler, President at West Chester University, PA, Professor Trudy W. Banta Senior Advisor to the Chancellor for Academic Planning and Evaluation at IUPUI, and Claus Nygaard, associate professor at Copenhagen Business School for sharing their quality practices. I would like to thank Professor Abbas Tashakkori for his generosity in sharing his work and the time spent on discussing my design. I also thank professors John Creswell and Tony Onwuegbuzie for the suggestions and insights graciously provided to improve my knowledge in Mixed Methods.

I thank Professor Brent Ruben from Rutgers University, for his excellent direction, advice, and expertise in Malcolm Baldrige Award for which his recommendations and suggestions greatly improved my work. I also want to thank Dr. Jani Ursin from University of Jyväskylä for sharing his questionnaire. I would like to thank professors in statistics George Marcoulides from California and Thodoros Chatzipantelis from Greece who provided me with consistently good advice in statistical matters. I wanted Professor Thodoros Oikonomou in Sociology, to be alive and I wanted to thank him and my friend Dr. Fotoula Karalidou, Philologist, for their help during the translation of my questionnaires from English to Greek. Of course I would like to thank my U.S. friends Dr. Diane R. Dean, Bill Higgins, and Anthony Petrokonis who offered me a wide array of support and my former students Pantelis Logginides and George Kampitsis.

My gratitude is extended to other members of CHEPS: Dr. Liudvika Leisyte for her friendship, support, generous hospitality and help me with my problems as external PhD, Dr. Grid Laudel for her generous hospitality and also Dr. Paul Benneworth and Drs. Frans Kaiser for their help. 
The Greek frame-law gives the chance to its university staff for lifelong learning via sabbaticals and leaves of absence with the department's approval. I am thankful to the colleagues of the Economic Department at Aristotle University who approved and supported my application to study abroad.

A word of gratitude also must go to all to the willingness of rectors vice rectors, department heads, and administrators who agreed to participate in the study while several were against its topic in that particular period.

Last but not least, a special word of thanks and appreciation goes to my editor, Bill Strickland, for always being there for me with his outstanding editorial comments, and for helping me to make this dissertation more readable.

Finally, I especially want to applaud the endurance of two people from New York who remain like family to me in this rough journey: Fred Lane, Professor Emeritus at Baruch College and my best friend, Dr. Aspasia Papadakis, for their help, patience, and encouragement. Professor Lane was always supportive by sending me articles and provided me useful information and sources. Professor Lane and Aspasia encouraged me, while at the same time they were constructively critical when needed. Professor Lane and Aspasia gave me the best gift because they believed in my abilities and me, they reassured me that I could finish my studies when difficulties made me want to abandon on my journey. I cannot thank you enough for your patience and never-ending support.

Antigoni Papadimitriou

Thessaloniki, December 2010 


\section{Contents}

List of Tables $\quad 15$

$\begin{array}{ll}\text { List of Figures } & 17\end{array}$

$\begin{array}{ll}\text { Abbreviations } & 18\end{array}$

1 Introduction $\quad 19$

1.1 The Rise of Quality Management in Higher Education 21

1.2 Studies about Quality Management in Greek Higher Education 25

1.3 Problem Statement and Research Questions 27

$\begin{array}{ll}1.4 & \text { Neo-institutional Perspectives } \\ 1.5 & 29\end{array}$

$\begin{array}{ll}1.5 & \text { Mixed Method Strategy } \\ 1.6 & 30\end{array}$

1.6 Plan of the Book 32

2 Quality Management in Higher Education Institutions 33

$2.1 \quad$ Quality in Higher Education 34

$\begin{array}{ll}2.1 .1 & \text { Quality Management } \\ 35\end{array}$

2.2 Different Types of Internal and External Quality Management 39

2.2.1 The EUA's Institutional Evaluation Programme 39

2.2.2 Malcolm Baldrige National Quality Award 43

2.2.3 ISO in Higher Education 49

2.2.4 Quality Management Models: Observations and Comparisons 52

2.3 Quality Practices from Abroad 54

2.3.1 Weaving Excellence at West Chester University, Pennsylvania 55

2.3.2 Systematic and Continuous Quality Enhancement at Copenhagen Business School (CBS)

2.3.3 Assessment Plan and Strategic Planning Approaches at Indiana University-Purdue University Indianapolis (IUPUI) 64

2.3.4 Planning and Institutional Improvement 64

2.4 Concluding Remarks and Lessons Learned 68

3 Environmental Challenges, Organizational Change and the Adoption of Quality Management: Building the Conceptual Framework 72

3.1 Universities as Organizations in an Open Systems Perspective 73

3.2 Neo-Institutional Organizational Theory 78

3.3 Neo-Institutional Theory in Higher Education Studies 81

3.4 Neo-Institutional Theory and Quality Management Studies 84

3.5 Conclusions: Developing a Conceptual Framework and Revisiting the Research
Questions

4 Operationalization $\quad 90$

4.1 Variables Presentation and Dimensions 90 
$\begin{array}{ll}\text { 4.1.1 Independent Variables } & 91\end{array}$

$\begin{array}{ll}\text { 4.1.2 Dependent Variable } & 96\end{array}$

$\begin{array}{lll}4.2 & \text { Research Methods } & 98\end{array}$

4.2.1 Mixed Methods Methodological Considerations 99

$\begin{array}{lll}\text { 4.2.2 Present Study's Design } & 101\end{array}$

4.3 Sources of Data and Methodical Approaches 106

$\begin{array}{lll}\text { 4.3.1 Content Analysis } & 106\end{array}$

$\begin{array}{lll}\text { 4.3.2 Survey } & 107\end{array}$

4.4 Ways and Criteria for Evaluating the Quality of the Entire Study 110

$\begin{array}{ll}\text { 4.4.1 During Data Collection } & 112\end{array}$

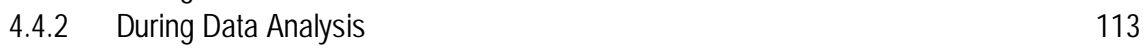

$\begin{array}{ll}\text { 4.4.3 During the Inference } & 114\end{array}$

$\begin{array}{ll}\text { 4.4.4 During Integration } & 115\end{array}$

5 Environmental Scan of Greek Higher Education 116

$\begin{array}{ll}5.1 \text { General Information } & 116\end{array}$

5.1.1 Legal and Political Issues in Greek Higher Education 118

$\begin{array}{ll}5.1 .2 & \text { Economic Elements } \\ 5.1 .3 & 125\end{array}$

$\begin{array}{lll}5.1 .3 & \text { European Elements } & 131\end{array}$

$\begin{array}{lll}5.1 .4 & \text { Technological Elements } & 133\end{array}$

$\begin{array}{lll}5.1 .5 & \text { Sociocultural Elements } & 138\end{array}$

$\begin{array}{lll}5.2 & 143\end{array}$

$\begin{array}{lll}\text { 5.2.1 Academic Personnel and Departments } & 144\end{array}$

$\begin{array}{lll}\text { 5.2.2 Decision-making } & 145\end{array}$

$\begin{array}{ll}\text { 5.2.3 Decisions Regarding Teaching and Research } & 146\end{array}$

$\begin{array}{ll}\text { 5.2.4 Leadership Positions and Senate } & 147\end{array}$

5.3 Summary and Conclusion 148

6 Media Coverage of Quality Assurance 152

$\begin{array}{lll}6.1 & 152\end{array}$

6.2 Theoretical Considerations and Media Coverage 153

$\begin{array}{lll}6.2 .1 & \text { Agenda-setting } & 153\end{array}$

6.2.2 Media in Agenda-setting 154

6.2.3 Greece and Agenda-setting around the 1999 Greek European Elections 154

6.2.4 Stakeholders and Neo-Institutional Pressures $\quad 155$

6.3 Methodology: Content Analysis of Greek Newspapers 156

$\begin{array}{ll}\text { 6.3.1 Operational Definitions and Categories } & 157\end{array}$

$\begin{array}{lll}6.4 \quad \text { Findings } & 159\end{array}$

$\begin{array}{lll}\text { 6.4.1 Newspapers Sampled } & 159\end{array}$

$\begin{array}{ll}\text { 6.4.2 Data Analysis } & 160\end{array}$

6.5 Neo-Institutional Pressures and Media Coverage 163

$\begin{array}{ll}\text { 6.5.1 Qualitative Analysis } & 163\end{array}$

$\begin{array}{ll}\text { 6.5.2 Quantitative Analysis } & 165\end{array}$

6.6 Summary and Conclusion 168

7 Which Universities Voluntarily Invite an External Evaluation? 172 
$\begin{array}{lll}7.1 & \text { Introduction } & 172\end{array}$

$\begin{array}{lll}\text { 7.1.1 Methodology } & 172\end{array}$

$\begin{array}{ll}7.2 \text { Findings } & 173\end{array}$

$\begin{array}{lll}7.2 .1 & \text { Sample } & 173\end{array}$

$\begin{array}{lll}7.2 .2 & \text { Data Analysis } & 174\end{array}$

$\begin{array}{lll}\text { 7.3 Analysis in Comparison to the rest of the } 21 \text { Universities } & 189\end{array}$

$\begin{array}{lll}7.4 & \text { Summary and Conclusion } & 191\end{array}$

$8 \quad$ Surveying of Quality Management via Malcolm Baldrige Education Criteria 195

$\begin{array}{lll}8.1 & 195\end{array}$

$\begin{array}{ll}\text { 8.1.1 Methodology } & 195\end{array}$

$\begin{array}{lll}8.2 \text { Findings } & 198\end{array}$

$\begin{array}{lll}\text { 8.2.1 Sample } & 198\end{array}$

$\begin{array}{lll}8.2 .2 & \text { Response Bias } & 199\end{array}$

8.2.3 Dealing with People who "Don't Know" 200

$\begin{array}{ll}\text { 8.2.4 University Characteristics } & 201\end{array}$

8.2.5 Greek Leaders Assess the Malcolm Baldrige Award Education Criteria 202

8.2.6 Hypothetical Malcolm Baldrige Scores for Greek Universities 204

8.3 In-depth Analysis: Construction of Diagnostic Items 206

$\begin{array}{lll}\text { 8.3.1 Neo-Institutional Pressures } & 206\end{array}$

$\begin{array}{lll}\text { 8.3.2 Leadership } & 207\end{array}$

$\begin{array}{ll}\text { 8.3.3 Quality Management Dimensions } & 208\end{array}$

$\begin{array}{ll}\text { 8.3.4 Analysis of Gaps } & 210\end{array}$

8.4 Summary and Conclusion 211

9 The Adoption of Quality Management: Perceptions of Department Heads 216

$\begin{array}{lll}9.1 \text { Introduction } & 216\end{array}$

$\begin{array}{ll}\text { 9.1.1 Methodology } & 216\end{array}$

$\begin{array}{lll}9.2 & \text { Findings } & 219\end{array}$

$\begin{array}{lll}\text { 9.2.1 Background Information } & 219\end{array}$

9.2.2 Familiarity, Usefulnes, and Applicability of a Quality Assurance System 219

9.2.3 Advantages and Disadvantages of a Quality Assurance System 221

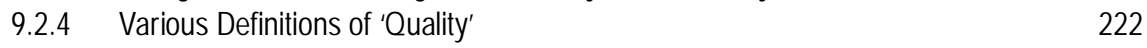

9.2.5 State of Development of Quality Assurance Systems 222

9.3 Pressures for the Adoption of Quality Assurance Systems 224

$\begin{array}{lll}9.4 & \text { Summary and Conclusion } & 227\end{array}$

10 The Use of ISO Standards in Greek Higher Education 231

$\begin{array}{ll}10.1 \text { Introduction } & 231\end{array}$

10.2 Methodology 232

10.3 First Phase: Finding Out the Extent of Use of ISO Standards in Greek Higher Education

$\begin{array}{lll}\text { 10.3.1 Findings } & 233\end{array}$

10.4 Second Phase: Isomorphic Pressures and ISO Standards 235

$\begin{array}{lll}10.4 .1 & \text { Survey Instrument } & 235\end{array}$

$\begin{array}{lll}\text { 10.4.2 Population Characteristics } & 237\end{array}$ 
$\begin{array}{lll}10.4 .3 & \text { Findings } & 237\end{array}$

10.5 Summary and Conclusion 242

\section{Integration and Reflection: Neo-Institutional Approach, Quality Management, and Greek Higher Education 245}

11.1 Introduction 245

11.2 Macro Level Views on Isomorphism and Quality Management 249

11.3 Meso Level Views on Isomorphism and Quality Management 253

11.3.1 Documented Quality Management 253

11.3.2 Perceptions about Isomorphism and Quality Management 255

11.3.3 Meso Level Views of Isomorphic Pressures: Leaders' and Department Heads'

Perceptions 260

11.3.4 Bringing together Isomorphism, Quality Management and University Characteristics262

11.4 Micro Level Views on Isomorphism and Quality Management 265

11.4.1 Department Heads' Perceptions and Concerns on Pressure and Adoption of Quality Assurance

11.4.2 Isomorphism and Use of ISO in Laboratories and Academic Support Services

11.4.3 Bringing together Isomorphism and Quality Management at the Micro Level in Greek Universities

11.5 Overall View Regarding Isomorphic Pressures and Adoption of Quality Management in Greek Higher Education 269

11.6 Reflections on Neo-Institutional Theory and Mixed Methods Methodology 272

11.6.1 Neo-Institutional Theory and Isomorphic Pressures 272

11.6.2 Methodological Aspects of a Mixed Methods Study 273

11.7 Future Avenues of Quality Management Research in Greek Higher Education 275

11.8 Navigating the Archipelagos of Greek Higher Education: Recent and Future

Challenges and Changes in Greek Higher Education 277

Nederlandstalige samenvatting $\quad 282$

Appendices $\quad 292$

$\begin{array}{ll}\text { References } & 317\end{array}$ 


\section{List of Tables}

Table 2-1 ISO standards description $\quad 50$

Table 2-2 Quality systems and important issues facing higher education 53

Table 2-3 Elements of comprehensive framework developed and formal quality models $\quad 54$

Table 2-4 Distributed Leadership principles $\quad 59$

Table 4-1 Morse's (2003) notations and abbreviations concerning mixed methods 101

$\begin{array}{ll}\text { Table 4-2 Quality criteria for interpretive rigor } & 115\end{array}$

Table 5-1 Universities' regular national budget 2004-2007 (million euro) 126

$\begin{array}{ll}\text { Table 5-2 Factors of the academics basic salaries } & 126\end{array}$

Table 5-3 Number of students and graduates of universities 139

Table 5-4 International students in Greek universities $\quad 141$

$\begin{array}{ll}\text { Table 5-5 The structure of leadership and decision-making } & 146\end{array}$

Table 5-6 Decision-making bodies and major decisions 147

Table 6-1 2005 monthly newspaper clippings on higher education issues in three databases 159

Table 6-2 Number of titles with "evaluation" 161

Table 6-3 "Evaluation" trends covered by Greek newspapers during May 2005

Table 6-4 Popular words in May 2005 headings 162

Table 6-5 Quantitative overall findings $\quad 165$

$\begin{array}{ll}\text { Table 6-6 Articles' tone during May } 2005 & 167\end{array}$

Table 6-7 "Locked" and similar terms in newspapers titles 168

$\begin{array}{ll}\text { Table 7-1 University characteristics } & 185\end{array}$

Table 7-2 Characteristics of the 13 non-EUA-IEP universities 189

Table 7-3 Types of universities by participating or not-participating in the EUA-IEP until 2006190

$\begin{array}{ll}\text { Table 7-4 By age } & 190\end{array}$

$\begin{array}{ll}\text { Table 7-5 By size } & 190\end{array}$

Table 7-6 By location $\quad 190$

$\begin{array}{ll}\text { Table 7-7 By range of studies } & 191\end{array}$

Table 8-1 The Seven MB Categories 196

Table 8-2 MB Categories and total number of questions cited in survey tool 197

Table 8-3 Response rates of EUA participants and non-EUA universities 199

Table 8-4 Characteristics of participants' universities 202

\begin{tabular}{ll} 
Table 8-5 Normative pressure & 207 \\
\hline
\end{tabular}

$\begin{array}{ll}\text { Table 8-6 Mimetic pressure } & 207\end{array}$

Table 8-7 Leadership triad and leadership excellence level 208

Table 8-8 Overall Quality management stages 209

Table 8-9 Quality management stages by university characteristics 209

Table 8-10 Average gaps between importance and implementation per university 210

Table 8-11 Gap overall analysis $\quad 211$

Table 9-1 Survey response by discipline and by university (absolute numbers) 218

Table 9-2 Familiarity, usefulness, and applicability of quality assurance systems (means) 220 
Table 9-3 Familiarity, usefulness and applicability of a quality assurance system by discipline (means)

Table 9-4 Familiarity, usefulness, and applicability judgments by stage of development of quality assurance (means)

Table 9-5 Perceptions of isomorphic pressures towards the adoption of quality management (absolute numbers)

Table 10-1 Use of ISO standards in Greek higher education

Table 10-2 ISO practices and type of isomorphic pressures

Table 11-1 Isomorphic pressures at macro level in Greek higher education system 252

Table 11-2 Leaders' perspectives on quality management stage at the meso level 256

Table 11-3 Leadership Excellence and QM stage at the meso level 258

Table 11-4 Department heads' perceptions about their universities quality management 258

Table 11-5 Overall quality management stage combining views from university leaders and department heads 259

Table 11-6 Overall quality management stage as derived from Chapters 8 and 9 surveys $\quad 260$

Table 11-7 Normative and mimetic pressures at the meso level, combining views from university leaders and department heads $\quad 261$

Table 11-8 Isomorphism and quality management at the meso level 262

Table 11-9 Quality management at the meso level in Greek higher education as related to university characteristics Age, size, location and range of studies 263

Table 11-10 Isomorphic pressures at meso level in Greek higher education system 264

Table 11-11 Isomorphic pressures at micro level in Greek Universities 269

Table 11-12 Types of isomorphic pressures on different levels of the Greek higher education system 270

Table 11-13 Inference quality answers 273 


\section{List of Figures}

Figure 2-1 MB framework $\quad 47$

$\begin{array}{ll}\text { Figure 2-2 Culture of Evidence at IUPUI } & 67\end{array}$

$\begin{array}{ll}\text { Figure 3-1 Organization as an open system } & 74\end{array}$

Figure 3-2 Conceptual Framework 88

Figure 4-1 Visual model of the procedures for the multi-level mixed design 103

Figure 5-1 Expenditure on tertiary education as a percentage of GDP in $1999 \quad 128$

Figure 6-12007 media users' satisfaction $\quad 157$

Figure 6-2 "Evaluation" trends for $2005 \quad 161$

Figure 6-3 "Evaluation" trends during May $2005 \quad 161$

Figure 6-4 Total frequencies of isomorphic pressures and their tone 166

Figure 8-1 Importance and Implementation rates (averages of 9 universities) 204

Figure 8-2 Hypothetical quality index for Greek universities 205

$\begin{array}{ll}\text { Figure 11-1 Conceptual framework } & 246\end{array}$

Figure 11-2 Presentation of the Empirical Studies 248 


\title{
Abbreviations
}

\author{
AACSB Association to Advance Collegiate Schools of Business \\ AASCU American Association of State Colleges and Universities \\ ACE Danish Accreditation System \\ ADIP Hellenic Quality Assurance Agency For Higher Education \\ AUTH Aristotle University Thessaloniki Greece \\ CBS Copenhagen Business Schools \\ CEMS The Global Alliance in Management Education \\ CEOs Chief Executive Officers \\ CRE Association of European Universities \\ DL Distributed Leadership \\ ECTS European Credit Transfer and Accumulation System \\ EFMD European Foundation for Management Development \\ EFQM European Foundation for Quality Management \\ EHEA European Higher Education Area \\ ELOT Greek Organization for Standardization \\ EMAS Environmental Management and Accounting System \\ ENQA The European Association for Quality Assurance of Higher Education \\ EQUIS European Quality Improvement System \\ ESMU European Centre for Strategic Management of Universities \\ EU European Union \\ EUA-IEP European University Association- Institutional Evaluation Programme \\ FEK Greek Official Journal \\ GDP Gross Domestic Product \\ HEIs Higher Education Institutions \\ ISO International Organization fro Standardization \\ IT Information Technology \\ IUPUI Indiana University-Purdue University Indianapolis \\ IWA International Workshop Agreement \\ MB Malcolm Baldrige National Quality Award for Education \\ MBNQA Malcolm Baldrige National Quality Award \\ MM Mixed Methods Research \\ OECD Organisation for Economic Co-operation and Development \\ POSDEP Hellenic Federation of University Teachers' Associations \\ QA Quality Assurance \\ QM Quality Management \\ QUAL Qualitative \\ QUAN Quantitative \\ R\&D Research and Development \\ SPRC Strategic Planning Resource Council \\ TQM Total Quality Management \\ UOM University of Macedonia Thessaloniki Greece \\ WCU West Chester University \\ YPEPTH Ministry of National [Greek] Education and Religious Affairs
}




\section{Introduction}

This study focuses on change and stability in Greek higher education as regards the introduction of quality management in universities. Thematically, this study is rooted in organizational research and management research on higher education, within which quality management practices are investigated as an instance of organizational change.

Quality in higher education, how to evaluate it, how to enhance it, andincreasingly so-how to manage it, has been placed high on the contemporary agenda in higher education. The literature from the late 1980s onward suggests a growing interest in quality management in the higher education sector and related issues on organizational change and stability.

Currently, quality management has become a buzzword among policy-makers and consultants, who assume that a more systematic and managerial approach in universities and colleges will help them to improve universities' performance. However, the actual capacity of the modern university to respond to change has remained an enigma (Johnson et al., 2003, p. vii). Perhaps the greatest challenge for the university in the Knowledge Age is determining how to balance its historic traditions and heritage with powerful societal forces for change. For example, Johnson, Hanna, and Olcot (2003), writing about change in the modern university, stated that vision for change must come from inside the institution, at the department and college levels. They argued that leadership, technology, and academic culture are interconnected dimensions of managing organizational change. They also argued that deans and chairpersons in universities must manage these dimensions concurrently if they are to create systematic change in their organizations. The authors confirmed what Clark (1983) stated earlier; changing higher education from above is very difficult, although there is a lot of bottom-up change. Blazey et al. (2003, p. 4) affirmed that for leaders, effecting changes in education is tough, stating that it is hard work to change an educational and learning system that has been relatively untouched and unchallenged for decades. "It is easier to move a graveyard than to change a curriculum", they observed.

Changing Greek higher education institutions is reputedly even more difficult. Bonikos (1998, p. 87) argued for example: 
Greek universities are notoriously rigid establishments that lack the flexibility institutions require to respond imaginatively and reasonably to new academic needs and priorities. Therefore, introducing change in a Greek university has always been a battle between status quo preservationists and evolutionary expansionists who welcome new forms.

Greece seems a good location for studying forces for change and stability in higher education, since quality management was introduced only recently into this higher education system; Greece, therefore, becomes an important place for my study.

Greece may be an attractive place for a holiday because of its rich history, food and nightlife, but recent news about its universities seems dominated by strikes, student marches, protests, and similar issues have eclipsed any focus on the quality of Greek higher education. Some views regarding quality assurance in higher education are found in the Greek National Reports published in the framework of the Bologna Process in 2003, 2005, 2007, and 2008 (YPEPTH, 2003, $2005,2007,2008)$. The reports claimed that the framework for operating a national system for quality assurance in higher education was under consultation before the Greek Parliament. This framework, however, only became an active law (ref: Law 3374/2005) in 2007. Related events involve the Hellenic Quality Assurance Agency for Higher Education's website, which went online in March 2007. How much adoption of quality management accompanied those messages is the question my book investigates.

This book examines a period during which quality assurance, evaluation, and quality management have a heavily debated meaning for the universities in Greece. Stamoulas (2006, p. 437) noted that "stakeholders were viscerally opposed in their particular ideas for the structure, scope, operation and the results of the evaluation". He also pointed out that the chairman of the Greek National Council, Veremis, stated that "there is a lot of reaction to evaluation because Greeks generally do not like evaluation. They shun all forms of quality assessment for themselves, while they are all too keen to evaluate others" (as quoted in Stamoulas, 2006, p. 437). Likewise, Papalexandris and Chalikias (2002, p. 345) in their survey regarding performance appraisal, commented that "in Greece, appraisal is based more than in the EU on the next level superior, while employee participation in the procedure is still considerably lower, something quite normal given the somehow negative attitude of employees towards performance appraisal". Such reactions to evaluations and appraisals are not all new and well understood in the higher education sector. In 1996 in an article in the Times Higher Education Supplement, Marseilles wrote that Greek university chiefs had 
reacted angrily to education secretary G. Papandreou's suggestions that future funding of universities should be based on student numbers and that teaching staff be assessed for promotion every five years by foreign professors. Yemptos, Chancellor of Athens University said: "The assessment of Greek teachers by foreigners is unacceptable even as an idea because it diminishes the value of Greeks, which are very high quality and recognized internationally". Metaxopoulos, chancellor of Pantion University, suggested that Greek prime Ministers (and perhaps education ministers) be assessed by a committee of three European prime ministers and in case of a negative report the country should have its subsidies reduced (Marseilles, in Times Higher Education Supplement, 2/8/1996).

The above comment reflects one part of the Greeks' approach towards evaluations, quality assurance, and quality management. The situation of Greek universities is one where changing expectations in their institutional environment clashes with traditional values. There is pressure to change and to adopt modern quality management methods. The strikes, slow decision-making processes in public policy, and the ongoing debates within academia reflect resistance against change. How do universities respond in such a situation? Do they adapt, and if so, how do they do that? With this in mind, the research underlying this book sought to map the state of adoption of quality management in Greek public universities and to look for underlying forces for change and stability.

\subsection{The Rise of Quality Management in Higher Education}

The issue of quality management within the higher education field has interested researchers during the last thirty years investigating higher education management in the US and in Europe (Banta, 1985, 1986, 1993; 2002; Bensimon, 1995; Brennan 1997; Brennan, de Vries, \& Williams, 1997; Cornesky, 1991; Dill, 1995; Green, 1994; Harvey, 1998; Keller, 1983; Neave, 1988; Ruben, 2004; Schwarz \& Westerheijden, 2004; Seymour, 1991, 1995; Seymour \& Collett, 1991; Sherr \& Lozier, 1991; Sims \& Sims, 1995; Van Vught \& Westerheijden, 1993; 1994; Westerheijden, 1999).

Quality management is usually defined as organized activities dedicated to improving and assuring educational quality (adapted from Massy, 2003, p. 159). Quality management is supposed to systematize a university's approach to quality instead of leaving it mainly to unmonitored individual initiative. Arguably, attempts to manage quality in universities in a more structured and systematic way emerged first in the context of the US higher education system and they were partly inspired by models and practices from the business world. 
After Total Quality Management (TQM) had become popular in the business world, quality management started to enter the US higher education during the early 1980s. Within US higher education, there are many small-sized, private universities; however, within that grouping, a significant number of them are oriented towards a business model of private corporations as opposed to many of the larger, individual state supported universities. The smaller, private schools have adopted professional management as a normal operating procedure (Clark, 1983). Williams (1993, p. 229) characterized the rise of TQM in universities as a "product of the market ideologies of the 80's and of the managerialism that accompanied it". The aims of introducing quality management techniques were mainly competition, cost containment, accountability, and service orientation (Seymour, 1991). Chaffee and Sherr (1992, p. 1) noted that in the US "external agencies and the public have lost confidence: We might be 'for' quality, but in many eyes we do not 'do' quality". Adopting quality management meant adopting new techniques for central managers. Sims and Sims (1995, p. 1) stated that "TQM is the process of continuous improvement using select tools, techniques, and training to guide decision making and to plan actions. The results are quality processes, products, and services and, thus, high levels of customer satisfaction". According to Coate (1990, p. 27), TQM is a "structural system for creating organization wide participation in planning and implementing a continuous improvement process that exceeds the expectations of the customers. It is built on the assumption that 90 percent of problems are process, not employee problems". He also noted that TQM is a logical evolution of management by objectives, strategic planning, and other management systems. Edwards (1991) mentioned that the major components of TQM-a documentable quality management system, statistical process control, and team work-can be applied to higher education on the initiative of the management team (both academic and non-academic) and with input from staff support areas.

This first wave of adopting quality management was not without problems. Jelinek, Foster, and Sauser (1995, p. 110) stated that "[e]ducational institutions have not embraced TQM as have business and industry, largely because of culture, structure and individual roles". The authors who Winter (1991) quoted, mentioned that "perhaps the most significant barrier to implementation of TQM is that higher education organizations already view themselves as participatory". Massy (2003, p. 165) considered that "the greatest resistance to quality process improvement comes from professors who think it's just another business-oriented fad", while Chaffee and Sherr (1992) mentioned that the faculty will play the most important role in developing the concept of continuous quality improvement and other ideas about TQM as they might apply to academic activity. Banta (1993, p. 144) believed that there is hope for overcoming the barriers to continuous 
improvement within the academy but noted that "nothing less than a culture change is required to do so".

Ewell (1991) analyzed the US assessment movement suggesting that assessment with its emphasis on outcomes and data may be a driving force behind change and improvement of quality in higher education. The assessment movement started in the USA in the 1980s, because state legislators and governing boards pressured institutions to be more "accountable" to the public that funded higher education; and on the other hand, major reports (i.e. such as A Nation at Risk, Integrity in the College Curriculum and Involvement in Learning) signalled the improvement of the quality of student learning (Ewell, 1991). The assessment movement's main achievements were primarily to improve students' learning and secondarily concerned accountability for the quality of learning produced. According to Angelo (1999, p. 1) "[al]though accountability matters, learning still matters most". The main criticisms levelled against the assessment movement came from faculty. Strada (2001, p. 188) found that "many professors actively engaged in assessment have expressed thoughtful criticisms regarding the current modus operandi. In particular, instructors lack confidence in assessment's relevance (applicability to classroom teaching and learning), validity (truly measuring learning outcomes), proportionality (institutional benefits of assessment commensurate with effort devoted to it), and significance (answering the question that comes naturally to academics: So what?)." Ewell (2005), one of the leaders of the assessment reform in US higher education mentioned that despite the longevity of the assessment movement, "four dilemmas [of] practice" still existed, i.e. the dilemma of purpose, of stance, of technique, and of consequences.

Gioia and Thomas (1996) as well as Schwarz and Westerheijden (2004) also pointed out that the use of practices and models for quality management originated in the business world; those models were adapted to be applied to higher education. Academic research on the rise of quality management in the USA contained, however, quite different views about the value of these processes and models. On the one hand, Dill (1995, p. 107) declared that "through Deming's eyes we can see that assuring quality in academic programs will require more than encouraging rational university choices by students, or providing positive incentives for faculty members to teach. It will also require re-weaving the collegial fabric of academic communities, the collective mechanisms by which faculty members control and improve the quality of academic programs and research". More recently, Rosa and Amaral (2007) presented an extensive review of the use of TQM as one of the models from the business world applied to the field of higher education. They claimed that "applying TQM principles, concepts, 
and tools in higher education is not an easy process or one exempt from critics" (p. 191). They argued, however, that TQM is a viable path for higher education institutions to follow if they wish to improve their quality continuously. On the other hand, Birnbaum's (2000) Management Fads in Higher Education analyzes the management models that one after another have moved across the US and other Western higher education systems as fads and portrayed TQM as the latest of these failed fads. In a similar vein, Temple (2005) criticized the European Foundation for Quality Management's (EFQM) Excellence Model as a classical fad.

The emergence of quality management in Europe has been inspired by developments in the USA; meanwhile, differences between continents on how governments and universities perceive quality management practices must be taken into account. Rhoades and Sporn (2002) showed that the terminology and the procedures of quality management underscored the significance of local adaptation between US and Europe. They found that the meanings of quality assurance and assessment, particularly as they related to the management of institutions, were quite different in the US from their counterparts in Europe.

Van Vught (1996) stated that the issue of quality had been brought to a much higher level of pertinence in Europe because of several developments in higher education in recent decades. The increase of public expenditure, the expansion of the Western European higher education system, the challenge to universities to explain what they were doing and how well they were doing it, the increased international mobility of students, teachers and researchers in Europe and the internationalization of the European market were developments that led to a growing need to understand the equivalence of qualifications, standards and credits in European higher education systems and therefore, to a growing need to pay more attention to quality assessment systems. Sporn (1999) analyzed how several environmental changes (restructuring the economy, the changing role of the state, shifting demographics, new technologies and increasing globalization) were strongly influencing demands of access, quality, cost, and effectiveness of education at colleges and universities. She emphasized that these new environmental demands triggered internal responses from universities around restructuring, retrenchment, re-engineering, (total) quality management, strategic planning, financial accounting, and technology transferred. Policy initiatives taken to improve the quality of higher education in Europe from the 1980s onwards were embedded in a wider perspective of the public sector reforms (Bleiklie, 1998; Neave 1988; Pollitt \& Bouckaert, 2000; van Vught 1989). The main elements of governmental reform have been to increase the efficiency (ability to perform), effectiveness (ability to fulfill political objectives), and accountability 
(ability to legitimize the results) of the public sector. The emphasis on efficiency, effectiveness, and accountability has forced higher education institutions to adopt structures, systems, mechanisms, and models intended to enhance such objectives. There is evidence from some countries that among the many external measures affecting higher education institutions during the 1990s, quality management "has proven to be the most potent of change agents" (Kogan \& Hanney, 2000, p. 240). This is also underlined by the fact that across Europe, quality measures have been linked directly or indirectly to the funding of universities to an extent not known in the US. Attention in research and practice thus shifted from policy-making to issues of policy implementation and organizational adaptation of quality management, i.e. trying to explain which environmental factors trigger change and stability in the adoption of quality management practices and trying to identify factors that help to explain why adoption succeeds or fails. These issues are investigated in this study and will be elaborated into a specific research problem and related questions in the following section. However, this study does not look at quality management in general, but specifically at quality management in the Greek higher education context. Therefore, a brief overview follows on the study of quality management in Greek higher education.

\subsection{Studies about Quality Management in Greek Higher Education}

Very few studies have been published concerning the Greek higher education system in general and quality management more specifically. Overall, the study of Greek higher education has neither been on the forefront of interest of social and political science research in Greece nor of the international literature in higher education studies. There are a few articles about Greek higher education in journals and sections in books, including the entry by Eliou on Greece in the Encyclopedia of Higher Education (Clark \& Neave, 1992, pp. 265-275), which provides an overview of the historical development and state of the art of Greece's higher education in the early 1990s. Eliou mentioned that "concern over the condition of higher education has been expressed for many years in OECD reports (1962, 1965, 1980, 1982, and 1984) which, however, have not impeded certain development[s]". In this connection, he cited Psacharopoulos (1988) and noted that problems hampering the external efficiency of the higher education institutions were: excessive politicization, excessive privileges, indiscriminate distribution of opportunities, curricular rigidity, excessive central regulation, too much student absenteeism and scantiness of funds for research". Eliou also discussed that the overall evaluation of Greek higher education might focus on the existence of a network of problems at three levels. At the first level, he mentioned that "the existing structure of secondary education, in conjunction 
with the infrastructural inadequacies of tertiary education, maintains a problematic system of entrance into IHEs and creates powerful social pressures which, in turn, contribute to the development of excessive central regulation and to student emigration". At the second level, Eliou noted that "the structural problems have effects on the quality of the education provided, which is marked by its cramping uniformity, the shrinkage of requirements in terms both of learning and of grading, and a type of high school rationale". At the third level, "problems created by the structure and quality of higher education are expressed in a number of ways - under the statutory cover of the laws-in the day-to-day running of the institutions: in excessive absenteeism among students; in shortcomings in the teaching of faculty members; and in indirect party-political intervention, which distorts the conception of participation, isolates the voice of the academic community, and maintains inertia". Like a scaffold, the problems mentioned by Eliou built on each other, and it seems each problem was designed to hamper the internal efficiency of Greek higher education institutions. In that way, Eliou's conclusion becomes plausible: “...in the extensive and substantive literature concerning higher education which is developing in Greece, two important points stand out: the need for radical intervention in higher education; and the need for this intervention to be prepared with specialised research on the one hand, and with consensus procedures, on the other, to ensure the widest possible support from the social and political spectrum" (Eliou, 1992, p. 274).

More recently, some publications have addressed issues of quality assurance and quality management in Greek higher education. Bourantas et al. (1996) provided a short text about traditional evaluation systems in Greek higher education and formal types of internal evaluation in the Athens University of Economics and Business (e.g., ad hoc student feedback and small studies on teaching load). Billiris (2004) contributed a short chapter to the volume "Accreditation and Evaluation in the European Higher Education Area" (Schwarz \& Westerheijden, 2004). It was a short chapter, because the author noted that evaluation and accreditation at that time had not been formally established in Greece. Regarding accounting reform in Greek universities, Venieris and Cohen (2004) published an article that explains why this reform, six years after its inception, had not yet seriously progressed. They analyzed this situation by using Pettigrew's (1977) theory of organizational change and decision making. The authors claimed that introducing the accounting reform had failed because it "was conceived in a narrow frame, with a little investigation of the effects of its application on the organizations involved and without profound reference to the problems and contradictions that might occur" (p. 201). One of the few articles on evaluation in Greek universities supported Sporn's findings (Politis \& Siskos, 2004). The authors stated that the survival of companies and organizations in a 
contemporary, demanding society made continuous improvement imperative and they pointed out that this was also the case in higher education. They emphasized that the rapidly evolving environment caused changes both in the framework conditions within which universities operated and in the expectations placed on them. Another voice regarding quality assurance, Stamoulas (2006) published his article, "Greece before Bologna Process: Confronting or Embracing Quality Assurance in Higher Education?" His article went beyond the limits of a strictly technical debate about the implementation of evaluation procedures or what its criteria ought to be, with the purpose of presenting the broader sociopolitical and economic background that influences the enactment and operation of quality assurance in Greece. The author claimed that "it is not clear though how Greece will be meeting the goals of the Bologna Process, and in moving on the reform front with the rest of Europe without re-engineering socio-political and economic conditions that hindered quality assurance in the past" (Stamoulas, 2006, p. 443).

Altogether, our brief review does not only point to the fact that the study of higher education in Greece is still a developing area of research, but extends to a general concern about the state of development and reform of Greek higher education and focuses specifically on the issue of 'quality'. This study thus hopes to make a significant contribution to further our understanding of the drivers and obstacles that might champion or impede change in Greek higher education; in consequence, this study provides a specific focus on the late emergence of quality management in the Greek higher education system.

\subsection{Problem Statement and Research Questions}

This study examines the adoption or non-adoption of quality management within the universities in Greece as outcomes of organizational change practices. The problem statement of this study can therefore be summarized: to identify relationships between the organizational factors for stability and/or change in Greek universities and the universities' adoption (or lack of adoption) of quality management. Dividing the problem statement into three sets of sub-questions will help make a more complete analysis of the problem. The first set of questions includes the conceptual considerations for this study. Therefore, the first part of the study involves developing our knowledge on quality management as used in higher education institutions.

1. What do we understand by quality management in higher education institutions? 
This question will be approached through a review of the conceptual literature as well as - to make it more application-oriented - through analyses of major quality management approaches and sustained cases of quality management in higher education institutions. The quality management approaches addressed include the European University Association-Institutional Evaluation Programme (EUAIEP), Malcolm Baldrige National Quality Award (MBNQA) and ISO 9000 (these abbreviations will be explained further in due course).

The following question generally addresses the factors and mechanisms identified in organizational theory and in higher education studies more specifically in order to build understanding about the adoption or non-adoption of quality management and how that plays out in (Greek) universities. This following question will be addressed as an issue of organizational theory:

2. Which organizational factors for stability and/or change concerning the adoption of quality management can we observe in the literature on organizations and higher education institutions?

I shall argue that the organizational factors should first be searched or scanned within the environment: the constantly changing environment exerts pressure(s) on higher education institutions to adapt. A basic assumption of this study is that organizations want to survive. According to Drucker (1995) all organizations operate from a theory of business that assumes the organization's environment is critical to accomplish the organization's survival. Drucker suggested that many businesses suffer problems because their theory of business no longer applies. This results from changes in the environment. Consequently, to survive, changes in the organization's theory of business are required. Higher education institutions are very different from businesses and their theory of business is different as well. Researchers describe universities as organizations with unique characteristics (Baldridge et al., 1977; Baldridge \& Deal, 1983; Dill, 1992). Some distinguishing characteristics that affect a university's decision processes regarding adaptation to change are goal ambiguity, client service, task complexity, professionalism, and environmental vulnerability. In addressing the issue, I attempt to identify the mechanism(s) through which organizations and in particular higher education institutions respond to such calls for change by making use of insights from neo-institutional theories. This theoretical approach will be given special attention in chapter 3 . Some preliminary observations on it will follow in the next section.

The empirical part of the study addresses the question of "if" and "how" these theoretical considerations are empirically useful to understand higher education 
in Greece. The research question that guides the empirical part of the study can be formulated as:

3. Which organizational factors for stability and/or change concerning the adoption of quality management can we find empirically in Greek higher education? Do these organizational factors differ at the macro level (higher education system), the meso level (individual universities) and the micro level (department, laboratory and academic support services)?

This study examines quality management in Greek universities at macro, meso and micro levels up to the year 2007. The reason for drawing this border was that in that year the context changed considerably when a new law was adopted regarding quality assurance in higher education, and consequently the national system for quality assurance in higher education made its first public appearance.

The final part of the study is related to whether the empirical observations and evidence validate or refute the theoretical insights gained in this study:

4. Does the empirical evidence on the adoption of quality management in Greek universities coincide with the theoretical approaches articulated in this study?

\subsection{Neo-institutional Perspectives}

The central concern of this study is to understand the adoption of quality management practices in public universities in Greece through the lenses of organizational theory. Neo-institutional theory has become one of the dominant approaches for explaining how organizations adapt to institutionalized pressures from their environments for change in their business theory. This study employs some core concepts of neo-institutional theory, applied to quality management in organizations.

The study of institutions is complex, not only because the nature of institutions is somewhat amorphous, but also because of the varying approaches to institutions in the different disciplines (Dill, 1999, 2003). From a neo-institutional view, organizations operate in an environment dominated by rules, requirements, understanding, assumptions, beliefs, and procedures (scripts) about what constitutes appropriate or acceptable organizational forms and behavior (DiMaggio \& Powell, 1983; Meyer \& Rowan, 1977; Oliver, 1991; Scott, 1987). Building on this argument, DiMaggio and Powell (1983) developed a widely used concept to identify institutional pressures for organizational change that captures 
the process of homogenization: isomorphism. They argue that isomorphism can emerge because non-optimal forms are selected out of a population of organizations or because organizational decision makers learn appropriate responses and adjust their behavior accordingly. DiMaggio and Powell (1983) identified three mechanisms through which isomorphic change occurs: coercive forces, which stem from political/egal influence and the problem of legitimacy; mimetic forces resulting from standard responses to uncertainty; and normative forces associated with professionalization.

Dill (2003) pointed out that the neo-institutional theory has great potential relevance to the study of academic quality assurance, and the concept of isomorphism has been applied in various studies on this issue. Schwarz and Westerheijden (2004, p. 5) stated, for example, that quality assurance as a separate instrument in university management started as a new management tool in industry that mimicked the success of the Japanese economy. Schwarz and Westerheijden interpreted this from a European perspective as both the old isomorphism drive to copy whatever seemed successful in US higher education and the new isomorphism drive to copy whatever seemed successful in industry. Rhoades and Sporn (2002) asked: "To what extent and through what processes have concepts of quality assurance and strategic management been borrowed from the US and adapted in European higher education?" They found that quality assurance practices emerged in the US through both mimetic and coercive processes of isomorphism, in which higher education was influenced by private sector and state government practices. In Europe, the same mechanisms operated through different structures: multinational business was a source of mimetic isomorphism (e.g. TQM); and national governments were a source of coercive isomorphism. In addition, they recommended that future research should explore in much greater detail the implementation of various quality assurance and strategic management practices and encouraged scholars to consider a wide range of conditions and effects.

\subsection{Mixed Method Strategy}

My study of quality management in Greek higher education employs several research techniques and data collection methods in order to obtain a full perspective on this issue. A mixed methods strategy seemed the most appropriate methodology for this study (Johnson \& Ownwuegbuzie, 2004; Ownwuegbuzie \& Teddlie, 2003; Tashakkori \& Creswell, 2007; Tashakkori \& Teddlie, 1998, 2003; Teddlie \& Tashakkori, 2009). A mixed methods strategy is defined as: "research in which the investigator collects and analyzes data, integrates the findings, and draws inferences using both qualitative and quantitative approaches and 
methods in a single study or program of inquiry" (Tashakkori \& Creswell, 2007, p. 4). Tashakkori and Teddlie (2003) suggested that variety of data sources and analysis is needed to understand complex social phenomena or realities thoroughly. In addition, Curall and Towler (2003) suggested that mixed methods are considered appropriate when the research questions concern process and dynamic phenomena such as innovation and change. I used several research techniques such as document analysis, surveys and interviews with a variety of respondents (i.e., rectors, deans, laboratories' directors and academic support services administrators) as well as observations. This study can be characterized as a multi-level mixed design using both concurrent and sequential data collection.

The mixed method strategy was also chosen for other reasons. First, the introduction of quality management in a higher education system is a complex issue that may look different at the different levels. Different levels may need studying in several different ways and the core theoretical approach of neoinstitutionalism contributes to realizing that need. For instance, Mizruchi and Fein (1999, p. 678) noted that the new institutional theory has become a leading perspective within organizational analysis. Additionally, they pointed out that problems arise in cases in which authors stipulate only one type of isomorphic process while ignoring alternate options that are equally possible. They stated that when authors assume that only voluntary mimicry accounts for an organization's behaviour, without considering alternative explanations, including coercion, they might be providing a limited and biased picture of the processes they are trying to analyze.

Another reason guided my choice in methodological matters as well. The hot debate that took place in Greece during the period while this study was being conducted made it difficult to study quality management directly. Studying a 'hot topic' is already a sensitive and difficult matter in the best of circumstances. But Greece is an environment that is internationally notorious for its difficulty for conducting empirical social science research: accordingly, very low levels of cooperation have to be expected (as also found by Bourantas et al., 1990; Bourantas \& Papadakis, 1996; Elefteriou \& Robertson, 1999; Makridakis et al., 1997; Psychogios \& Priporas, 2007; Spanos et al., 2002), while only a few empirical studies report good access to the field and high response rates in surveys (e.g. Gotzamani \& Tsiotras, 2001; Lipovatz et al., 1999; Papadimitriou \& Westerheijden, 2008). For all of these reasons, a sophisticated approach to the empirical part of the study was necessary. 


\subsection{Plan of the Book}

Following this introduction, the book is organized in 10 further chapters. Chapter 2 will address conceptual issues and approaches to quality management in higher education based on a literature review as well as via an exemplary perspective on sustained cases of quality management in higher education institutions. Chapter 3 discusses the theoretical perspective and provides a summary presenting the conceptual framework for this study.

The variables enclosed in the conceptual framework will be operationalized in chapter 4 . This chapter will set out the key variables and their operationalization. It will introduce the methodological considerations in relation to the mixed method strategy. It will also outline the specific qualitative and quantitative approaches and will conclude with criteria for evaluating the quality of the entire study.

The empirical research question is addressed in a multi-level mixed design. Chapter 5 will provide the necessary background knowledge on the Greek higher education system and its environment, using the categories set out in chapter 3 . The macro level of the higher education system as a whole will furthermore be addressed in chapter 6 by researching the media's view of quality management in higher education in 2005 (when the law adopted in 2007 was first published). Chapter 7 provides a meso-level picture, at the level of separate higher education institutions, of the studied phenomenon and is derived from eight evaluators' reports, as until 2006, eight Greek universities participated in a European programme of evaluations (EUA-IEP). Chapters 8 and 9 present findings at the meso and micro levels based on concurrent quantitative and qualitative surveys addressing rectors' and deans' perceptions of quality management and pressure for adoption for their institutions (i.e. meso level) and departments (micro level). Chapter 10 also presents findings at the micro level. It was a sequential qualitative study examining the use of ISO standards in Greek higher education laboratories and academic support services, and the type of pressure that individuals felt in order to adopt this particular quality management practice.

Chapter 11, the final chapter, is where the different empirical studies will be drawn together, leading to more general inferences as well as to considerations and reflections on the study as a whole. In addition, the chapter provides a brief excursion to recent reforms and changes in Greek higher education. 


\section{Quality Management in Higher Education Institutions}

This chapter explores approaches to quality management in higher education. Changes have taken place in the organizational environment of higher education institutions worldwide. In order to confront this new environment, it has been a widely held view by experts and political authorities that universities should adopt new forms of organization to acquire the strategic capacity to adjust and meet the needs of the outside world in an independent, structured, and coherent manner (Clark, 1998; Davies, 1997). These pressures, amongst many other consequences, have led to a growing emphasis on clear and systematic mechanisms for quality management in the universities. Significant efforts are underway to improve the quality of the higher education offered to students; the concepts and applications of quality management are critical to these efforts.

Against the background of institutional change, the key question is not so much a technical one of how to implement quality management activities, but rather, what are the rationales of quality management activities and what their (expected) effects. Some authors claim that an external perspective on quality management is driven by increasing demands for accountability, while others claim that an internal perspective of organizational learning drives change. Obviously, these perspectives can be linked in theory as well as in practice. They are also interrelated with different basic perspectives on "quality" such as stressing an output-oriented view on products, stakeholders, cost effectiveness, or a process-oriented view that embraces the transformative aspects of organizational improvement.

The literature offers a number of analytical concepts or synoptic definitions, and it is worth exploring some of those in this study. Further, a selection of practical approaches regarding higher education quality management will be presented and discussed; namely, the European University Association (formerly CRE) with its Institutional Evaluation Programme (EUA-IEP), the Malcolm Baldrige Education Criteria for Performance Excellence (MB), and the ISO certification.

In the final part of this chapter, I will present and discuss three real-life examples of quality management in universities. Such examples are not only useful for practitioners who want to adopt or adapt them, but they also add extra value to this study, in which I want to examine similar issues. I had the opportunity to study different philosophies and practices of universities to judge and improve their quality and to learn more about some of the intra-organizational factors that 
turned out to be crucial for the quality management practices within those universities.

\subsection{Quality in Higher Education}

Quality is a core value in higher education and it is increasingly associated with quality assurance and quality management practices. At the same time, quality is a slippery and vague concept for which it is still difficult to find agreement on a single definition, regardless of its increasing popularity in higher education policy and practice (van Vught, 1996; Stensaker, 2004).

For example, Birnbaum (1988) defined quality from the perspective of purpose and requirement by taking into consideration the view of stakeholders. He pointed out three dimensions of quality in higher education: the meritocratic (the institution's conformity to professional and scholarly norms with academic professionals as a reference group), the social (the degree to which an institution satisfies the needs of important collective constituents), and the individualistic (the contribution the institution makes to the personal growth of students). Harvey and Green (1993) took an empirical route to identifying how different stakeholders in higher education perceived the term quality. They identified five broad categories that represent stakeholders' views on quality: quality as excellence, quality as zero errors, quality as "fitness for purpose" (mission orientation, consumer orientation), quality as a value for money, and quality as transformation.

Kristoffersen et al. (1998) claimed that a procedure for the evaluation of quality in higher education must be in line with the concept of quality that one chooses. They proposed a concept of quality as a sophisticated version of the "fitness of purpose" concept. More specifically:

- quality in higher education needs to be defined in light of specific purposes

- these purposes must be suited to higher education systems

- different categories of customers (or stakeholders) hold legitimately different opinions; academic excellence is one of these opinions

- $\quad$ as the primary users of higher education, students are an important category of customers

- with mass higher education, students' needs become ever more varied 
- for these reasons, "purposes" are best defined at the level of individual higher education institutions, faculties, or study programs, taking into account the national context.

Pollitt and Bouckaert (1995) argued that in principle there are only two major perspectives on quality in public management including higher education, an output-oriented view including definitions such as value for money, stakeholder satisfaction and zero errors, and a process-oriented view embracing quality as transformative, which serves organizational learning and improvement. The output-oriented view of quality can be linked to political movements in public sector reform inspired by New Public Management approaches with their emphasis on deregulation of public services, greater managerial discretion, the introduction of market mechanisms, and a focus on stakeholders' needs and satisfaction.

Those who stress organizational learning and a transformative view have argued that emphasis should be taken away from an external product and stakeholder orientation while focusing more on the internal processes and stakeholders within higher education institutions (see e.g. Dill, 1995). In the context of the present study, it is argued that these views are not necessarily mutually exclusive and can be combined conceptually notwithstanding tensions and dilemmas in real-life practices of quality management.

In sum, the concept of quality has given rise to controversies in trying to look at a variety of phenomena while the maintenance and improvement of quality has become a crucial issue in the field of higher education. It seems impossible to give a definition of quality upon which all experts or stakeholders would agree; meanwhile, it is not the intention of this study to add another definition of quality to the existing perspectives. But rather, one should note an ongoing struggle between output-oriented and process-oriented views and related external and internal perspectives. Such struggles are not only academic in nature but reflect real-life conflicts about the understanding of "quality" in higher education and the related issue of the meanings and purposes of quality management.

\subsubsection{Quality Management}

Generally speaking, the term quality management refers to all the activities that contribute to defining, designing, assessing, monitoring, and improving the quality of an organization, field, or individual organization, specifically in the field of higher education or an individual university. Quality management thus deals with the policies, systems, and processes designed and implemented to 
ensure the maintenance and improvement of quality. Quality management is supposed to be a means to an end or to multiple ends of improving the quality of all the different services provided. For example, Campbell et al., (2000) defined quality management as a system in order to ensure the quality and future development of all the activities of a university including teaching and learning, research, European and international policy, administrative and management functions, and academic recognition practices. The authors claimed that quality management activities need to be integrated with other institutional decisionmaking and planning processes. They argued that the purpose of this quality management practice is to allow the institution to learn about itself, to know itself, to make improvements and changes where necessary, and to interact effectively with the external environment, both nationally and internationally. However, they underscored that managerial practices do not have a tradition in many continental European higher education institutions, where there had been both greater dependency of universities on governmental steering and a high degree of autonomy of faculties.

Quality, which for a long time has been assumed as an "ineffable abstraction in academe" (Csizmadia, 2006, p. 24), thus began to be regarded as something that can be managed and pro-actively improved in the latter part of the twentieth century. Thus, the rise and implementation of quality management activities do not take place in a vacuum. One important factor concerns the purpose of quality management that is often divided into accountability and improvement. Most external quality management systems were first established for the purpose of accountability to external stakeholders. On the other hand, quality management systems are also established for the purposes of internal improvement and accountability. Campell et al. (2000) observed that the existence of external quality requirements has been a major impetus behind the establishment of internal quality management systems. The relative weight of the two goals of improvement and accountability thus differs in the practices of different countries and institutions (van Vught, 1996, p. 195). Empirical research on the implementation of quality practices tends to show that if universities engaged in quality assurance voluntarily, the effectiveness tended to be much more marked than when they complied with government-initiated policies (Brennan \& Shah, 2000). In this context, Gaither (1998, p. 87) observed that "the most successful quality assurance programs are initiated, maintained, and enhanced through the professional commitment of the faculty, not through quality assurance systems, administrative controls, or legislation". In other words, quality management practices in universities can been seen as responses to external pressure, such as governmental quality assurance policies, or internal policies towards organizational learning and performance improvement. Schwarz and 
Westerheijden (2004, p. 6) mentioned that "spontaneous serious involvement of universities in quality assurance without governmental policies were rare exceptions". Prominent exceptions were the "dozens of universities that volunteered for the CRE's Institutional Evaluation Programme".

Another issue concerns the argument that achieving sustained quality education requires organizational change to infuse the organization with a culture of quality. In this perspective, I could refer to Colling and Harvey (1995) who asserted, for example, that systems and procedures of quality control, assurance, and assessments only make sense in higher education institutions 'enterprises' if the link to continuous improvement is clear and unambiguous. They emphasized that to have any meaning in the day-to-day operations of universities, quality systems must depend on people. Therefore, the purpose of external scrutiny is to encourage staff and students to carry out their respective roles and responsibilities in support of each other and thereby enhance the quality of the "product" of higher education. Gaither (1998) noted that in industry, quality is a managerial task; however, in academia it is a professional faculty issue that requires professional commitment, generally of a non-authoritarian, nonadministrative nature. External participants as well as internal professionals are involved in internal quality assurance processes that should be continuous, active, and responsive.

Another issue that is frequently addressed concerns the role of leadership and management for the implementation and facilitation of quality management. Campbell et al. (2000) underscored that a major and frequent challenge is the need to foster leadership and management capacities at the institutional level, not just through increasing competences (i.e. knowledge, skills, and professionalism) but also through motivation. The authors also noted that "until a strong cadre of professional administrators is established, the professionalism and continuity required by complex organizations like higher education institutions will not be realized" (p. 48). Brennan and Shah (2000) suggested that in the changing environment, strong institutional management and leadership is needed because of the greater complexity of the external environment and the need for faster decision-making to affect the changes essential to ensure future institutional success and survival. While Burns (1978, p. 425) argued that "leadership is the reciprocal process of mobilizing, by persons with certain motives and values, various economic, political, and other resources, in a context of competition and conflict, in order to realize goals independently or mutually held by both leaders and followers". Additionally, for Lewis and Smith (1994) leadership is the enabling catalyst for successful intervention at the strategic management level. 
Here, we need to consider that rectors in most continental European universities, including the Greek ones, are elected professors who rotate back to the faculty, in contrast to the US or the UK, where presidents and vice-chancellors are permanent administrators appointed by a board and are like corporate CEOs (Green, 1997; Rhoades \& Sporn, 2002). Trow (1985) stated that academic CEOs generally have more power and authority than elected rectors. Furthermore, Senge (1990, p. 289) pointed out that the leadership role in learning organizations centers on leaders being "designers, teachers and stewards", while Green (1997, p. 35) asserted that the limited terms of elected rectors can also hamper their effectiveness. Green (1997) also mentioned Trow (1985) who observed that the ability of rectors to exercise leadership is limited not only by their method of selection, but also by the complicated structures within the university in which members of decision-making councils are expected to act as representatives of their constituencies. Furthermore, Simmons (1997) argued generally that effective leaders (not just in higher education) have understood that their job is to lead a process of systematic organizational transformation, and in order to be fully effective, this organizational transformation must focus on three areas: managing the future in a turbulent environment, improving productivity and quality, and building an inclusive organization. According to Simmons (1997, p. 274), "the basic job of the leader is to win the energy and commitment of people at all levels of the enterprise towards improving the performance of the organization as a system". The author argued "this means they [leaders] will need to develop their ability to release the intelligence, creativity, and initiative of people throughout the organization, particularly those people who have been traditionally ...[educated - AP], and then to integrate those initiatives towards an agreed vision of the future and to solving the problems encountered on the way" (p. 274). University leadership is characterized as a crucial topic in establishing an institutional quality management system by many scholars (e.g. Clark, 1970, 1998; Csizmadia et al., 2008; Keller, 1983; Newton, 2002; Rhoades \& Sporn, 2002, Sallis, 1994; Trow, 1985). However, Weber (2008, p. 263) argued that "even the most visionary and charismatic leader cannot set the direction [of the capacity to change] alone; he/she needs help to govern its/her institution". The author noted that this help "comes obviously from colleagues co-leading the institution with him or her and through the use of powerful management tools" (p. 263), which goes to say that leadership is seen here as a function which can be performed by several individuals (rector, vice-rectors, etc.) and that the possibilities of modern tools like quality management should not be ignored if the leadership wants to be successful in a present-day university. 


\subsection{Different Types of Internal and External Quality Management}

This section presents and discusses, in the final subsection, selected models of quality management and their use in higher education, namely the EUAInstitutional Evaluation Programme, the Malcolm Baldrige National Quality Award, and ISO 9000. These models, like others, and their use in the field of higher education have been objects of debate. For example, Gioia and Thomas (1996) argued that models reflecting Total Quality Management and/or Continues Quality Improvement approaches can be useful for higher education institutions and their improvement. They stated that universities have successfully adopted practices that have worked in business responding to changing expectations in their institutional environment. Rosa and Amaral (2007, p. 191) presented an extensive review of the literature and use of TQM. They claimed that "applying TQM principles, concepts, and tools in higher education is not an easy process or one exempt from critics". They argued, however, that it is a possible pathway for higher education institutions to follow.

Other authors have taken a more critical stance towards quality management models in higher education. Birnbaum (2001) reviewed seven higher education management fads, including TQM, which one after another have spread across the field of higher education in recent decades and which, as he showed, were eventually abandoned. In a similar vein, Temple (2005) criticized the EFQM Excellence Model as a classical fad in Birnbaum's sense. Others have argued that formal quality management models are not appropriate for academia and not focused on core teaching and learning processes (Grant et al., 2004; Vazzana et al., 2000). Ruben (2006, p. 4) referred to the generic tension between management approaches and higher education: "The term management and others such as strategic planning, marketing, productivity, and organizational effectiveness - and the concepts associated with them - are anathema within the academic community".

The literature surrounding quality management models in the higher education field indicates that their application is debatable. The main scope of the following part is not to end this debate; rather, it is to highlight selected models of internal and external quality management, which are used by universities worldwide and to set the stage for the reader to better understand some of the survey tools used in the empirical part of this study.

\subsubsection{The EUA's Institutional Evaluation Programme}

This section presents an overview of the quality assurance mechanism, namely the Institutional Evaluation Programme (IEP) developed mostly for European 
universities by the European University Association (EUA) formerly CRE1 ${ }^{1}$ Until 2010, 250 universities from 39 countries, mostly in Europe, have taken part in this evaluation program; among them were eight universities in Greece.

Hofmann (2005) claimed that the Institutional Evaluation Programme is a cornerstone of EUA's strategy to develop strong universities for Europe. When the IEP was launched in 1993, Sursock and Amaral (2008, p. 37) stated, "we may consider the strategy adopted by the CRE as an example of what Neave (1995; 1996) calls the law of anticipated results: institutions try to guess what will be required by government policy [and] act in anticipation". CRE (now EUA) piloted its own quality assurance procedure in 1993 and 1994 (van der Wende \& Westerheijden, 2001). Van Vught and Westerheijden (1996, p. 27) presented the IEP's goal to the member universities as: "the CRE institutional quality evaluation programme is to offer members of the association an approach and a mechanism that can help improve the institution's quality management process". Thus, the objective of the IEP was primarily an improvement-oriented, self-governing tool, wherein its evaluation procedure claims to be a tool for change - within higher education institutions in order to meet new challenges of the $21^{\text {st }}$ century. The EUA-IEP is marketed as a service to the EUA member universities as a supportive, external review, and as a tool for strategic and quality development. Van der Wende and Westerheijden (2001, p. 240) stated, "the IEP is not an institutional accreditation, but expressly development-oriented".

The EUA-IEP is thus a voluntary and supportive mechanism primarily focused on improving universities' capacities for strategic planning and internal quality monitoring. The EUA-IEP is characterized by the following points, which represent the EUA's basic philosophy²:

- a combination of an institutional approach with a European and international perspective through its experienced European teams (current and former rectors and an academic secretary)

- a focus on the self-evaluation process

- a philosophy as a supportive rather than judgmental procedure that takes into account the specific institutional and national context

- an emphasis on the strategic management of change.

The aim of EUA-IEP is to offer universities an external evaluation that takes into account each university's external and internal environment. The EUA-IEP

\footnotetext{
${ }^{1}$ Detailed information regarding EUA can be found at http://www.eua.be

2 Ibid. Last visit 1 April 2010.
} 
evaluates current conceptions of strategies and activities, while promoting internal quality in the universities. The basic questions of the EUA-IEP are:

1. What is the institution trying to do?

2. How is the institution trying to do it?

3. How does the institution know it works?

4. How does the institution change in order to improve?

Sursock and Amaral (2008, p. 38) pointed out that the abovementioned questions are based on a "fitness for purpose" approach. The authors noted that these "deceptively simple but strategic questions are meant to encourage universities to approach the institution strategically and critically during the self-evaluation phase". The EUA-IEP process is based on institutional self-evaluation, which is followed by two visits of peers whose intent is to learn, understand, and advise where possible. EUA-IEP's focus is on the context and mission of the university and examines its policy, strategy, and the institutional management tools and methods being utilized. The central actors in the evaluation teams are university rectors and presidents, who have both knowledge of and experience with different European higher education systems (the peer review) (Sursock \& Amaral, 2008). Peer review reports followed formal structures and guidelines in order to provide similar types of recommendations to individual universities. The EUA-IEP evaluators' reports are the main output of their review. Rosa and Amaral (2008, p. 74) noted that these reports "constitute an extremely relevant and important tool for universities to engage in quality improvement initiatives, trying to correct the main weaknesses identified by evaluators, reinforce their strong points, overcome their threats and take advantage of all the opportunities put before them". Therefore the authors noted that the evaluators, in order to facilitate producing reports as useful and valuable tools for universities, should provide realistic and achievable recommendations as well as emphasize the issues specific to each institution they evaluate.

It is also part of the philosophy of EUA-IEP that the quality journey begins-but does not end-with the participation of a university in the program. Accordingly, participating universities are encouraged to participate in a follow-up process to take up the evaluation results and recommendations actively. Rovio-Johansson et al. (2008, p. 58) discussed the follow-up activities "as a maturing process of IEP and also as a strategy to help institutions in its change process by offering a new impulse for change that might be necessary taking into account the supportive nature of IEP, its voluntary nature and the IEP's lack of enforcing mechanisms". 
Amaral (1998), in his comparative article on the US institutional accreditation system and the CRE's IEP, pointed out that the term "voluntary" in the US accreditation system can be misleading "because students enrolled in unaccredited institutions are not eligible for federal student-aid funds and because the reputation of an institution will be improved by obtaining accreditation from the appropriate bodies". Because of that, the US institutional accreditation is marked by what I shall call later in this book a coercive and mimetic pressure rather than by EUA's normative nature.

Sursock and Amaral (2008, p. 43) reported that the EUA-IEP was used in a variety of contexts and carried out evaluative tasks such as: Portuguese medical education and clinical training and the relationships of medical faculties to university hospitals; universities in Serbia, Bosnia, Herzegovina, Ireland, Catalonia, and Slovakia; and a sample of institutions in Portugal); polytechnics in Portugal and military and police academies in both Portugal and Slovakia. These examples demonstrate the "capacity of a relatively simple but flexible evaluation instrument to be useful in a variety of contexts" (Sursock \& Amaral, 2008, p. 44).

Additionally, Sursock and Amaral (2008) commented that the EUA-IEP had a great influence on quality assurance policy discussion at the European level. The authors stated how

the solid experience accrued in the Programme has enabled EUA to develop and articulate a policy position that argues that quality assurance procedures should meet several diverse goals (i.e. i. to promote innovative and dynamic institutions in a context characterised by diversity of missions, goals and curricula; ii. to preserve and extend institutional autonomy while meeting the demands of accountability; iii. to avoid a big bureaucracy, burdensome quality assurance mechanisms and promote cost-effective quality assurance procedures (p. 44).

In my eyes, the effect that the EUA-IEP had on the quality assurance policy discussion is related to its professionalization.

Another important issue addressed by the EUA-IEP is that its approach "aims to strengthen institutional autonomy and support quality awareness and institutional change in universities where it is needed" (Sursock \& Toft Jensen, 2008, p. 65). Universities that participated in the EUA-IEP have learned how appropriate it is to describe their everyday practices. Sursock and Toft Jensen (2008) underscored that the EUA-IEP has a formative orientation and therefore "has placed a singular emphasis on the self-evaluation process". The authors emphasized how "learning [is] considered the most important stage in an 
evaluation" (p. 66). The EUA-IEP Guidelines encourage broad ownership of the report rather than keeping it limited only to the top leadership (Surscok \& Toft Jensen, 2008, p. 66). It seems that this process of "learning by doing" has been warmly welcomed by university staff and faculty. Sursock and Amaral (2008, p. 39) noted that "the self-evaluation is not meant to simply produce a selfevaluation report but to trigger an internally driven change process".

Weber (2008, p. 268) discussed how the EUA-IEP "is more than an instrument to improve the quality of an institution in the traditional sense, but also a powerful tool to help an institution to improve its capacity of change in response to the rapidly changing environment in which it operates". However, he stated that the EUA-IEP has no control if universities follow up on the evaluators' comments and recommendations: "this is probably the weakest part of the approach as there is no control if the institution examines honesty the report and then doesn't try to implement changes" (p. 267).

Besides, out of the 250 universities that the EUA mentions in its publications, almost half participated because their country's respective Ministry of Education invited the EUA-IEP; since the invitations did not originate at the university level, it is not clear if these universities actually welcomed the process.

\subsubsection{Malcolm Baldrige National Quality Award}

Achieving performance excellence in higher education is also a challenge to many institutions. The Malcolm Baldrige Education Criteria for Performance Excellence (hereafter MB) were designed to help organizations use an aligned approach to organizational performance management that results in delivering everimproving value to students and stakeholders, contributing to marketplace success, improving overall organizational effectiveness and capabilities, and fostering organizational and personal learning. Blazey et al. (2003, p. xi) pointed out that "MB and related scoring guidelines are powerful assessment instruments that will help leaders of educational organization identify strengths and key areas for improvement".

In 1999, The Malcolm Baldrige National Quality Award for Education modified the business criteria for the education sector to search for educational performance excellence (Ruben, Lehr \& DeAngelis, 1999). Seymour (1996) stated that for higher education, the $\mathrm{MB}$ may be one of the best chances for the continuance of self-control of colleges and universities. He declared that MB "provides a methodology such that our professionals can correct deficiencies heretofore ignored; it is both a systematic and systemic way to regain control over 
our own institutions" (p. 11). The MB holistically looks at institutions in order to provide them with a vehicle to reflect on and articulate who they are and how they benefit their stakeholders. The seven categories of the criteria provide a systematic view of the institution, which is a prerequisite to institutional performance excellence. The criteria are meant to help the institutions align resources and approaches; improve communication, productivity, and effectiveness; and achieve strategic goals. The criteria also provide a framework that can help the institution plan in an uncertain environment. The MB is typically embraced as a way to conceptualize and plan for quality in the sense of excellence.

The Criteria for Performance Excellence are the basis for organizational selfassessments, for making awards, and for giving feedback to applicants. In the 2005 MB publication, it was affirmed that the Criteria have three additional important roles:

- to help improve organizational performance practices and capabilities

- to facilitate communication and sharing information on best practices among organizations of all types

- $\quad$ to serve as a working tool for understanding and managing performance, and guiding planning and training.

The seven categories of the MB are as follows:

1. The Leadership Category examines how universities' senior leaders address their organizational values, directions, and performance expectations, as well as focuses on students and stakeholders, student learning, faculty and staff empowerment, innovation, and organizational learning. Also examined are a university's governance and how a university addresses its public and community responsibilities.

2. The Strategic Planning Category examines how a university develops strategic objectives and action plans. Also examined are how a university chose strategic objectives and action plans, how they are deployed, and how progress is measured.

3. The Student, Stakeholder, and Market Focus Category examine how a university determines requirements, expectations, and preferences of students, stakeholders, and markets. Also examined are how a university builds relationships with students and stakeholders and how it determines the key factors that attract students and partners 
and lead to student and stakeholder satisfaction, loyalty, and persistence, and to increased educational services and programs.

4. The Measurement, Analysis, and Knowledge Management Category examines how a university selects, gathers, analyses, manages, and improves its data, information, and knowledge assets.

5. The Faculty and Staff Focus Category examines how a university's work systems and faculty and staff learning and motivation enable faculty and staff to develop and utilize their full potential in alignment with university's overall objectives and action plans. Also examined are a university's efforts to build and maintain a work environment and a faculty and staff support climate conducive to performance excellence and to personal and organizational growth.

6. The Process Management Category examines the key aspects of university's process management, including key learning-centered processes for educational programs, offerings, and service that create student, stakeholder, and organizational value. It also includes key support processes. This category encompasses all key processes and all work units.

7. The Organizational Performance Results Category examines a university's performance and improvement in key areas: student learning results; student- and stakeholder-focused results; budgetary, financial, and marketplace performance; faculty and staff results; operational performance; and governance and social responsibility. Also examined are performance levels relative to those of competitors and comparable organizations.

The Leadership (Category 1), Strategic Planning (Category 2), and Student, Stakeholder, and Market Focus (Category 3) categories represent the leadership triad. These categories emphasize the importance of a leadership focus on strategy, students, and stakeholders. Senior leaders must set organizational direction and seek future opportunities for their universities. If leadership does not focus on students and stakeholders, universities as a whole may instead focus on matters that are unimportant to students and stakeholders or even contrary to their needs and requirements.

A university's faculty and staff are the key processes used to accomplish the work of the organization that yields its performance results. Faculty and Staff Focus (Category 5) and Process Management (Category 6) comprise the work core-the people and processes that produce the Organizational Performance Results (Category 7). Significantly, Ruben (personal communication, 2007) observed that 
the Category 6, Process Management, was the most difficult category to understand for MB users.

Finally, the Measurement, Analysis, and Knowledge Management Category (Category 4) examines how an organization selects, gathers, analyzes, manages, and improves its data, information, and knowledge perceived as appropriate resources in understanding the quality management practice for an organization. Blazey et al. (2003, p. 149) noted how "this category is like the 'motherboard' on a personal computer. All information flows into and out of it". Moreover, the authors claimed that this category is the "brain centre" for the alignment of the organization's operations and its strategic objectives. Information and analytic processes are critical to the effective management of the organization and to management by objectives system for improving organizational performance and competitiveness. Furthermore, this category contains the technological environment associated with quality management practices in universities.

Sorensen, Fuste-Bowe, and Moen (2005, p. 3) described the whole process from their personal experience. They reported that educational organizations may use MB criteria "for internal self-assessment, or they may address the criteria in a 50page application and submit the application for external review, scoring, and award consideration". The authors pointed out that "each application is evaluated and scored by a team of trained examiners who have backgrounds and expertise in a variety of sectors" (p. 3). The authors also mentioned that "highscoring applications go on to receive a site visit by the examiner team to verify and clarify the information included in the application". Sorensen et al. (2005, p. 3) noted that "a panel of highly qualified judges selects the award recipients each year. Regardless of how many points an application scores, each applicant receives a comprehensive feedback report detailing key themes as well as organizational strengths and opportunities for improvement in each of the seven categories".

The MB criteria provide a systems perspective for managing an organization and its key processes to achieve results-or in other words, performance excellence. Figure 2-1 presents the framework connecting and integrating the categories. Ruben (2007) stated that the language and definitions used to describe the framework have changed over the years and vary somewhat from sector to sector. In 1993 a group of Academic Quality Consortium institutions decided to investigate if the $\mathrm{MB}$ criteria and items could work as a robust system for performance improvement for higher education. From the beginning, an effort was made for the $\mathrm{MB}$ to use language that was familiar to educators and to avoid the offensive language of 'customer', 'product' and 'supplier' without diluting the 
values of the criteria (Moore, 1996). As Seymour (1995, p. 29) reported "a language problem becomes a major detractor when you spend more time talking about language than talking about continuous improvement". The seven basic themes, however, remain constant. Ruben (2007) observed that further evidence suggests that the MB provides a valuable gauge of organizational effectiveness. Overall, he declared that there is a good deal of evidence to suggest that organizations rating highly on Baldrige standards are more successful than others, supporting assertions that the MB criteria provide a standard of excellence to which organizations can and should aspire. Ruben (2007) underscored its flexibility as one of the great virtues of the $\mathrm{MB}$ and therefore, he believed that the MB has been adopted-but also adapted-for assessment in a variety of organizational settings.

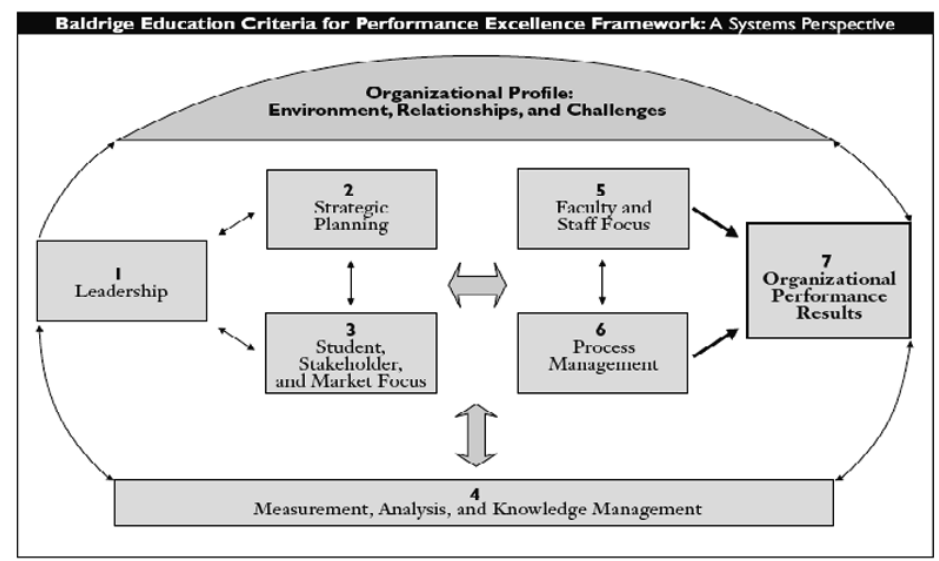

Figure 2-1 MB framework

Source: NIST, 2005

Conyers and Evy (2004) in their book about the 2003 award winning School District 15, characterized the MB criteria as "non-prescriptive". They wrote: "The criteria do not tell you what to do-you have the leeway to chart your own course. What MB does is give you a systematic framework for organizational excellence. How you bring it about is dependent on your leadership".

Ford and Evans (2000) compared the strategic planning category of Criteria for Performance Excellence against the scholarly literature. Their findings suggested some validity for the Criteria for Performance Excellence framework, which demonstrated the translation of research into managerial practice. 
Papadimitriou and Tsiotras (2007, p. 868) maintained that "the MB criteria can be used as a best practice for quality improvement of an educational organization and especially for its self-assessment report. The criteria might help universities or educational organizations-after appropriate practice and experience-to improve their performance and effectiveness and to organize their attention at focusing on quality"3.

Although it is popular in some academic circles, the MB has also met considerable criticism in, amongst others, articles comparing awards and quality management models. Rao Tummala and Tand (1996) cited Garvin (1991) and Sims et al. (1992) who mentioned three major deficiencies:

1. the award requires enormous expenditures on the application and preparation for site visits giving rise to the notion that $\mathrm{MB}$ Award "can be bought"

2. the award does not reflect outstanding or even exceptionally good product or service quality

3. the award fails to predict a company's competitiveness and financial success.

Loomba and Johannessen (1997, p. 59) investigated the MB "in order to discover the internal dimensions which delineate their inherent traits and qualities, and their natural limitations" by focusing on the ethics and ethical aspects of the MB. The authors argued that "what many critics fail to realize is that the primary value of the Baldrige Awards lies in the process-the road map provided by the criteria set-rather than in the handful of awards distributed each year" (p. 72). They also mentioned that quality guru, Juran, believed that "most of the criticisms have been misplaced by the media. The Wall Street Journal and Fortune have fallen into the trap of believing that filling out the application is the most important story, [...] It's not. Meeting the criteria is the heroic effort" (p. 72). However, the authors concluded in their study that they had "shown that the majority of the criticisms and ethical limitations of the Baldrige Award are negligible compared to the benefits it conveys to American businesses in terms of enhanced quality and productivity" (p. 76).

Another element that invited criticism of the MB was mentioned by Kumar (2007, p. 251): "there has been some criticism about the examiners of the MBNQA also working as consultants to organizations trying for the MBNQA". However, Kumar (2007, p. 251) also noted that "the MBNQA examiners can consult to

\footnotetext{
${ }^{3}$ All quotes from Greek sources are translated by the author.
} 
organizations but they cannot be examiners and consultant to the same organization". In other words, the MB does maintain a distinction between the roles of examiner and consultant, so that the criticism does not seem to apply very strongly. After all, one would want to choose experts of the criteria as examiners rather than outsiders. There is an unavoidable balancing act between the professional strength of the $\mathrm{MB}$ and its independence.

Ruben (2006, p. 6) claimed that "the Baldrige model has been a popular framework for organizational self-assessment within the United States". He stated "education, business, engineering, and health care [are] the standards for accreditation of college and university programs [which] have come to mirror the Baldrige framework in many respects". He also noted that institutional accreditation agencies in the US, such as North Central Associations of Schools and Colleges, Middle States Association of Schools and Colleges, and the Southern Association of School and Colleges, in their own frameworks also emphasize issues central to the MB framework. The MB therefore is in line with accreditation practice-and thus is not alien to higher education-it also emphasises the process for colleges and universities to gain academic achievement and improvement.

\subsubsection{ISO in Higher Education}

ISO 9000 standards set out to establish, document, and maintain an effective and efficient management system that could demonstrate an organization's commitment to quality and its ability to satisfy "customers" or requirements. This system refers to a series of standards for quality management. Its core module provides quality systems for design, development, production, installation, and services. According to its design, ISO 9000 provides a framework without changing how an organization operates. The ISO 9000:2000 quality management system consisted of a set of quality standards that have been revised and improved from the previous, 1994, version in order to apply better to a wider variety of organizations. Since the 1990s, the objectives of the ISO have changed from a model for quality assurance to a set of standards for effective quality management. Hoyle (2003) stated that the 2000 revision of ISO 9000 focused on the aspect of the management systems that will deliver customer satisfaction and continuous improvement to the system through objective evaluation.

The central organization of ISO supports the development of official guidelines for the implementation of the ISO 9000 standards. The guidelines universities would have to turn to are called the "International Workshop Agreement 2 
Quality Management Systems for the Application of ISO 9001:2000 in Education" (IWA). The purpose of the IWA was:

to provide guidelines to assist organizations that provide educational products to implement an effective quality management system that meets the requirements of ISO 9001:2000.... and to help educational organizations to relate the concepts in ISO quality management system standards to education practices (IWA, 2003, p. 1; as cited in Thonhauser, 2005, p. 17).

The previous standards of ISO 9001:1994, ISO 9002:1994, and ISO 9003:1994 have been integrated into the ISO 9001:2000. Recently ISO 9001:2008 replaced ISO 9001:2000 (see www.iso.org). However, the purpose of this presentation is not to cover in detail the integration process of ISO through the years, but rather to familiarize the reader with the research tools that will be used in the empirical part of this book. My study took place before and including 2006. Thus, in this subsection, I do not include specific information about ISO 9001:2008. Table 2-1 presents descriptions of ISO standards that were used in Greek higher education institutions until 2006.

Table 2-1 ISO standards description

\begin{tabular}{|c|c|}
\hline ISO & DESCRIPTION \\
\hline ISO 9000 & $\begin{array}{l}\text {-a guideline for selection and use of quality system standards } \\
\text {-provides insight for various situations and conditions as well as definitions } \\
\text { and explanations }\end{array}$ \\
\hline ISO 9001 & $\begin{array}{l}\text {-defines minimum quality system requirements for design/development, } \\
\text { production, installation, and servicing } \\
\text {-most complete standard } \\
\text {-applies to manufacturing and service businesses engaged in all these } \\
\text { activities }\end{array}$ \\
\hline ISO 9002 & $\begin{array}{l}\text {-essentially a subset of } 9001 \\
\text {-applies only to production and installation activities }\end{array}$ \\
\hline ISO 17025 & $\begin{array}{l}\text {-specifies the general requirements for the competence to carry out tests } \\
\text { and/or calibrations }\end{array}$ \\
\hline EMAS & $\begin{array}{l}\text {-recognizes and rewards those organizations that go beyond minimum legal } \\
\text { compliance and continuously improve their environmental performance } \\
\text {-participating organizations are required to regularly produce a public } \\
\text { environmental statement that reports on their environmental performance } \\
\text {-this voluntary publication of environmental information, whose accuracy and } \\
\text { reliability has been independently checked by an environmental verifier, gives } \\
\text { EMAS and those organizations that participate enhanced credibility and } \\
\text { recognition }\end{array}$ \\
\hline
\end{tabular}

Sources: www.stragetgosinc.com, www.emas.org.uk 
Moreland and Clark (1998) discussed the development of ISO 9000 certified quality assurance systems in three educational institutions in England: a university, a college of further education, and a primary school. Their analysis was conducted around three perspectives: sense-making and ISO 9000 in education, ISO 9000 and managerialism in education, and managing discourses to develop consent to ISO 9000. The authors reported that it was clear in their cases "that changing the practice of staff and management and the language with which they talk about the organization and work can make them view the idea of quality assurance and working to procedures seem a common sense-approach" (p. 314). In each of the three institutions in Moreland and Clark's research, the decision to develop a quality assurance system certified by ISO was a management decision involving little or no consultation with the staff: "Managers have procedures to work to, against which they can be audited" and "staff considered that the quality assurance system was an imposition from above" (p. 316). The authors noted that "putting the system into operation, is likely to provoke mixed reactions among staff, especially as the system can have social cost. For this reason the change to the standards has to be managed to make it most acceptable and to develop behavioral consent among staff" (p. 319).

Thonhauser (2005) conducted a comparative study in US and UK educational institutions that used ISO 9000. She identified factors that relate to the successful implementation of ISO 9000 in education, such as the importance of management commitment to ISO 9000, the previous existence of an unwritten or unspoken quality management practice, and a highly regarded management representative. Another important factor for successful ISO implementation is the status of the educational institution before they began to apply ISO 9000. Thonhauser's (2005, p. 77) findings indicated that US and UK education institutions are very similar with regard to ISO 9000 and that they implemented this quality assurance system for similar reasons-namely, to improve school efficiency, to have ISO 9000 as a marketing tool, to respond to pressure from industry to provide more skilled workers, and to adapt to a changing socio-economic environment.

Singh and Sareen (2006) argued that the main purpose of the ISO 9000 standards in education is to provide confidence to professionals, students and their parents, employees, and various stakeholders. In their empirical research, by obtaining data from twenty-one Indian higher education institutions (16 engineering, 2 management, 2 polytechnic colleges, and 1 medical institute), they found that the role of external consultants was very important. Most of the institutions had hired external consultants and took less than one year for certification. Secondly, they found that institutions improved documentation and maintenance records. Thirdly, they discovered that ISO 9000 was highly beneficial to systems, faculty, and students. Fourthly, they also reported that institutions faced problems during 
ISO 9000 certification such as scarcity of time and commitment and lack of obligations. The authors reported that institutions felt that ISO standards were time-consuming and paper-intensive and more meetings and documentation were required. In addition, the authors noted that "most faculty members were frustrated at the time spent away from classrooms commenting, 'more paperwork, less class time' and felt like they already had full workloads" (p. 411).

\subsubsection{Quality Management Models: Observations and Comparisons}

Thus far, this chapter has briefly explored conceptual issues and models of practice concerning the issues quality management in higher education, namely the EUA-IEP, the Malcolm Baldrige Education Criteria for Performance Excellence, and the ISO 9000 standards. These models have been used by universities worldwide either for improvement, for accountability, or for both, and for a variety of other purposes. Harvey and Green (1993) discussed how the "coin turns" to the universities to define their quality purpose and measurement method and to this extent quality management practices are a part of the "measurement rules".

The major purpose for EUA's IEP and MB is to promote awareness of quality and to increase the university's competitiveness. Leadership, strategic planning, and the organization's management system reflect the core values found in these models. Continued improvement and development play a major role in planning and implementing quality-directed efforts that satisfy the EUA's IEP and MB criteria. These improvement-oriented strategies call attention to any organization's recent overall accomplishments.

On the other hand, the ISO standards seek to establish, document, and maintain an effective and efficient management system that is able to demonstrate an organization's commitment to quality and an ability to satisfy its customers' requirements. By design, the ISO provides a framework for examination without changing the organization's basic operation. Participation by every individual, or presentation of a strategic plan, is not essential in satisfying the ISO requirements. Management involvement is, however, necessary during the process in order for the organization to establish and maintain a well-documented system, and this documentation is needed to certify that the organization is producing good services or products that its customers desire. In contrast, the leadership, people involvement, and strategic planning factors are an important part of EUA's IEP and MB. Leadership involvement, which has been characterized as the most crucial factor in implementing quality improvement, is absent from the ISO requisites. 
Izadi, Kashef, and Stadt (1996) compared the three major quality systems for industry, namely, the Malcolm Baldrige Award, the Deming Prize (not discussed here because of its more limited focus), and ISO 9000. They noted that the Baldrige Award is the most far-reaching and broad-range source of standards, that the Deming Prize adds numerous opportunities for sophisticated statistical analysis, and that ISO 9000 examines details at operating levels and entails periodic review and global recognition. The following Table 2-2 portrays the three quality systems and related important issues facing higher education institutions.

Table 2-2 Quality systems and important issues facing higher education

\begin{tabular}{lll}
\hline System & Focus & Important Issues for Higher Education \\
\hline $\begin{array}{l}\text { Baldrige } \\
\text { Award }\end{array}$ & $\begin{array}{l}\text { Customer } \\
\text { Satisfaction }\end{array}$ & $\begin{array}{l}\text { Customer Satisfaction and Retention } \\
\text { (i.e., Students, Employees, Parents, } \\
\text { Alumni, Taxpayers) }\end{array}$ \\
$\begin{array}{l}\text { Deming } \\
\text { Prize }\end{array}$ & Statistical methods & $\begin{array}{l}\text { Institutional Research and Assessment } \\
\text { (i.e., Enrolment Patterns, Student Progress, } \\
\text { Faculty Performance, Drop-out Rates, } \\
\text { Recruitment Activities) } \\
\text { ISO 9000 }\end{array}$ \\
& Documentation & $\begin{array}{l}\text { Acreditation and Evaluation } \\
\text { (i.e., Curriculum Analysis, Program, } \\
\text { Requirements, Facilities Analysis) }\end{array}$ \\
\hline
\end{tabular}

Csizmadia (2006) developed a comprehensive quality management framework that he used to compare EFQM, ISO 9001, 9004 and the Hungarian "Protocol". The results of his comparison are depicted in the following Table 2-3.

Csizmadia argued that some elements of ISO 9004:2000 and EFQM are compatible with his comprehensive framework. He also observed the near absence of quality management systems, design of student examinations, quality information systems, and external influencers in the EFQM model. Csizmadia also discussed how the comprehensive model calls for more emphasis on policy and strategy, educational process, quality information systems, and external influences than the ISO provides. Csizmadia ultimately claimed that his comparison demonstrated that the educational process is treated less than satisfactorily in all models compared.

EUA-IEP, MB, and ISO 9000 are frameworks of quality criteria or requirements for auditing quality systems. All these models remain non-prescriptive, in others words, these models assume that higher education institutions will define their 
own ways for achieving excellence in higher education. These ways could be different; however, ISO and MB have standards and criteria compared to which the organizations were assessed. EUA-IEP recognizes the complexity of universities as organizations in Europe, and bases its evaluation process on the formulated mission, the vision and the goals that individual universities want to accomplish.

Table 2-3 Elements of comprehensive framework developed and formal quality models

\begin{tabular}{|c|c|c|c|c|}
\hline \multirow{2}{*}{ Quality management framework } & \multirow{2}{*}{ EFQM } & ISO 9001 & ISO 9004 & \multirow{2}{*}{ Protocol } \\
\hline & & \multicolumn{2}{|c|}{2000} & \\
\hline Quality management system & 0 & ** & ** & 0 \\
\hline Policy and strategy & ** & 0 & 0 & 0 \\
\hline Learning outcomes & * & 0 & * & * \\
\hline Design of curriculum & * & 0 & * & 0 \\
\hline Design of education processes & * & * & * & 0 \\
\hline Design of student examination & 0 & * & * & * \\
\hline Implementation quality & * & * & * & 0 \\
\hline Resource management & ** & * & * & 0 \\
\hline Quality information system & ** & * & 0 & 0 \\
\hline Commitment of leaders & ** & ** & ** & ** \\
\hline Synergistic collaboration & ** & * & ** & ** \\
\hline Satisfaction of stakeholders & ** & * & ** & ** \\
\hline Demands (need research) & ** & 0 & ** & ** \\
\hline External influences & 0 & 0 & * & * \\
\hline Governing processes & ** & ** & ** & ** \\
\hline Support processes & ** & * & ** & 0 \\
\hline Organisational outcomes & ** & ** & ** & ** \\
\hline
\end{tabular}

0 : weak or not mentioned; *: treated to some extent; **: treated extensively

Source: Csizmadia (2006) with permission

\subsection{Quality Practices from Abroad}

This section presents three quality management practices from two US universities and one European university. This section is included as a pilot test for putting quality management theories into practice. Information on these cases was derived from interviews, site visits, university documents, and e-mails.

In most quality management models previously mentioned, the role of the leader was identified as crucial to the success of quality management. This element is 
emphasized in the quality model pioneered by President Madeleine Adler, Weaving for Excellence at the West Chester University of Pennsylvania (WCU), US. Its success was largely the result of her leadership and could be fully institutionalized due to her long tenure as president (Adler, 2006, personal communication). Although WCU's quality assurance practice does not follow the models mentioned above, this practice employs a blend of several issues reviewed previously.

The second quality practice examined here was the Systematic and Continuous Quality Enhancement at Copenhagen Business School (CBS), Denmark. The reason for selecting this practice was principally the notion of quality that CBS applied has adopted Harvey and Green's (1993) concept of quality that mentioned earlier; and in 2005, CBS also was awarded the prize as the Nordic university with the best work in the area of quality.

Thirdly, I investigated the role of assessments in the strategic planning at the Indiana University-Purdue University Indianapolis (IUPUI), US; the objective was to understand how the link between strategic planning and quality practices works in this university, where they are a long-standing good practice.

\subsubsection{Weaving Excellence at West Chester University, Pennsylvania}

West Chester University (WCU) is a public US university with some 13,000 students, along with 1,500 faculty and staff in the suburbs of Philadelphia, PA, U.S. It is the second largest of fourteen institutions in Pennsylvania's State System of Higher Education. It is a regional, comprehensive, multipurpose university offering degrees in the arts and sciences, advanced-study preparation for fields such as medicine and law, teacher preparation and certification, education for specific professions, and continuing education. While about $55 \%$ of the University's students are from the greater Philadelphia region, more than $70 \%$ of WCU graduates will accept their first job in the Philadelphia metropolitan area. For fall 2005, WCU received more than 11,000 applicants vying for the 1,850 openings available in the freshman class. Responses to the annual UCLA survey of freshmen revealed that WCU was the first choice of two-thirds of freshmen entering WCU, and the University's academic reputation was the most frequently cited reason for students having selected WCU4.

4 The Freshman Survey, Cooperative Institutional Research Program, Higher Education Research Institute, University of California, Los Angeles, reports to West Chester University of Pennsylvania, various years, 2003-2007. 
WCU's Vision is to be a national model of excellence for public regional comprehensive universities. WCU's Mission is committed to providing access while offering high-quality undergraduate education, select post-baccalaureate and graduate degree programs, and a variety of educational and cultural resources for its students, its alumni, and the citizens of Pennsylvania.

President Madeleine Wing Adler was appointed in 1995 as the $13^{\text {th }}$ President and the first woman to lead WCU. Dr. Adler underlined the importance of leadership by stating, "organizational success is rarely sustained without having motivated people and structures that promote meaningful change and innovation" (Adler, 2006, personal communication). She mentioned that the foundation for WCU's success is based on five principles: Leadership, Civility, Partnerships, Excellence, and Planning. Furthermore, she stressed that "WCU has three strong looms on which to weave their excellence". The first of these is their mission as a regional comprehensive university; the second is their strategic plan, The Plan for Excellence; and the third is their campus-wide distributed leadership.

\subsubsection{WCU's Mission as a Regional Comprehensive University}

According to President Adler, the label "regional comprehensive university" describes best the mission of the WCU and its place in US higher education. The university is considered "regional" because regional universities are "stewards of place". President Adler said: "as stated in the AASCU (American Association of State Colleges and Universities) web-site, "we [WCU] engage faculty, staff, and students with the communities and regions we serve - helping to advance public education, economic development and the quality of life for all with whom we live and who support our work. We affirm that America's promise extends not only to those who come to the campus but to all our neighbours" (Adler, 2006, personal communication). Dr. Adler said: "We serve our regions as 'comprehensive' universities. The university is committed to providing access and offering high-quality undergraduate education, select post-baccalaureate and graduate programs and a variety of educational and cultural resources for its students and alumni and the citizens of the region" (Adler, 2006, personal communication). She pointed out that they were able to achieve their mission by offering curricula in four professional colleges (College of Business and Public Affairs, College of Education, College of Health Sciences and the College of Visual and Performing Arts) as well as a broad spectrum of curricula offered in the College of Arts and Sciences. For example, she mentioned "students can major in Philosophy or Biology, Nursing or Business, Theatre or Piano performance, Early Childhood Education or Political Science. The curriculum of the university is broad and comprehensive" (Adler, 2006, personal communication). 
After that, I posed the question "How does all of that help to "weave excellence"; what is so strong about this loom?" and she responded accordingly:

WCU's motto is Expect Excellence. That motto is applied across the board. Students should expect to receive the finest undergraduate education at WCU. The community should expect to be served with the finest graduates in the region. The faculty and staff should expect to work and serve in an environment that respects diversity, differences of opinion and individual and team effort. We weave this into our culture by adhering to the Strategic Plan, The Plan for Excellence (Adler, 2006, personal communication).

\subsubsection{Plan for Excellence}

The second loom weaving WCU's advantage is their strategic Plan for Excellence. The Plan for Excellence, which was completed in 2001, focuses on the following five cornerstones of transformations:

1. Responsiveness - enhancing the University's capacity to meet the educational and cultural needs of the region

2. Student Success - making student success the institution's defining characteristic

3. Diversity - improving diversity, access, and equity, and fostering a climate that nurtures a multicultural community of students, faculty, and staff

4. Human Capital - investing in the skills and knowledge that faculty and staff need in order to be outstanding teachers, scholars, innovators, and leaders

5. Resourcefulness - diversifying physical and fiscal resources, and increasing the effectiveness of institutional management.

President Adler believed that these priorities will guide future University decisions and activities while ensuring that WCU can take its place among America's leading educational institutions. She underscored the need for effective planning in order to "provide clear direction, a yardstick for assessing progress and a safeguard against operational gridlock". Over the past 15 years, WCU has witnessed the development of a comprehensive planning process that emphasizes institutional mission, provides input from all campus constituencies, offers realistic appraisals of the internal and external environments, outlines specific objectives related to resource allocation, and sets measurable outcomes. Dr. Adler observed that "while many planning documents gather dust on bookshelves, the University's Plan for Excellence supports everyday activity as well as long-term goals". 
In response to my question: "How do you know that WCU is being served well by the Plan for Excellence?", President Adler replied that "A Plan for Excellence has served the University well for the last five years (2001-2006) and continues to do so; the Periodic Review Report, however, provides the opportunity for assessment of the Plan and its goals". The Strategic Planning Resource Council (SPRC) was established as a strategic plan monitoring committee, submitting annual reports to the President's Cabinet. Thus, the process and structure of this Periodic Review Report were also based on the Plan for Excellence, and SPRC has played a key role in information gathering and analysis. Several activities are meant to ensure campus-wide dissemination, understanding, and implementation of a Plan for Excellence.

\subsubsection{Distributed Leadership Model}

The third WCU loom is its distributed leadership model. President Adler stressed that among the many factors influencing institutional success, leadership is one of the most crucial. Prevalent literature reveals as abovementioned, that without strong leadership, an institution cannot progress significantly or sustain its achievement. However, many WCU documents highlight the way in which President Adler has instituted "distributed leadership" at WCU. It is built upon a management philosophy that emphasizes individual responsibility and accountability at all levels. Adler (Adler, 2006, personal communication) summarized as follows: "When I became president of WCU, I kept in mind what I had learned, and I stayed with the management style that matches my values and responsibility. My way of leading is based on a concept that I call Distributed Leadership (DL). I value this approach because it allows a university's vision for the future to be shared across the campus, rather than to be simply my vision or that of the top administration. DL is really an attitude more than anything else. It means seeing all employees as experts in their own right-as unique sources of knowledge, experience, and wisdom". She continued: "DL recognizes that the individuals who perform the various tasks within the University are those best suited to recommend and make improvements. Leadership, then, becomes the shared right and duty of all members of the organization rather than the prerogative of one individual or a limited few". Adler further explained that under DL, everyone is responsible and accountable for leadership within his area. Table 2-4 presents the principles of Distributed Leadership (Adler, 2006, personal communication).

For DL to work, four conditions must exist. DL demands, first, a culture of trust and mutual respect; second, well-informed team members who are themselves trained in leadership skills; and third, a context of clear values. 
Table 2-4 Distributed Leadership principles

1. Distributed Leadership does not mean delegating. Instead, it means finding the best path by tapping the expertise, ideas, and effort of everyone involved.

2. Distributed Leadership brings success in handling problems, threats, and change. It not only encourages idea sharing; it demands it. Good ideas can come to fruition because a team is ready to initiate the process that moves concept to reality.

3. "The way we've always done things" isn't necessarily the best way. Using Distributed Leadership, we can look for better ways and test them through controlled, reasoned risk- taking.

4. In a Distributed Leadership environment, mistakes often lead to discovering valuable new approaches.

5. In Distributed Leadership, not everyone is a decision-maker, but everyone is an expert whose knowledge contributes to the decision-making process.

6. Distributed Leadership is not for mavericks and lone eagles.

7. Distributed Leadership is about cooperation and trust, not about competition among units and factions. We all share the same mission, even though we contribute to it in different ways.

8. Distributed Leadership empowers everyone to make his job more efficient, meaningful, and effective.

9. Under Distributed Leadership, everybody matters.

Distributed Leadership practices are being extended throughout the levels of the University by modeling the practices, providing direction and support to managers, and institutionalizing Distributed Leadership as part of the University's organization. This management philosophy has led to the development of WCU's decentralized budgeting environment and allocation models, which enable vice presidents, deans, and directors to make more effective use of fiscal resources. President Adler's final comment was "admittedly, transformation presents challenges. Nevertheless, the path to excellence will require that University community to think and act differently. In order to achieve the Plan for Excellence transformations, the Plan must guide all aspects of campus life" (Adler, 2006, personal communication). 


\subsubsection{Systematic and Continuous Quality Enhancement at Copenhagen Business School (CBS)}

The second case of quality practice is derived from the Systematic and Continuous Quality Enhancement at Copenhagen Business School, Denmark. In 2005 a Nordic Project was set up as a comparative analysis of Nordic Higher Education Institutions' systematic quality assurance work. The primary objective of the project was to support the Nordic higher education institutions in developing systematic internal quality work and share good practices of quality work between them. The Project Group highlighted that aim because it was not only to support the higher education institutions involved in the project, but also others by sharing the good practices through their report. In 2005, CBS was awarded the prize as the Nordic university with the best work in the area of quality (Nygaart \& Kristensen, 2009). The quality system of CBS is presented here as "an outstanding example of best practices". "CBS has demonstrated quality work that undoubtedly fits in well with the institution's own personal culture, context, and purposes" (Omar \& Liuhanen, 2005, p. 27).

CBS, established in 1917, is one of the largest institutions of higher education and research at the university level in Denmark and is among the largest business schools in Europe. CBS offers broad perspectives on business studies and research, ranging from social science to the humanities. CBS's mission statement indicates that "CBS wants to be among the best institutions of higher education in Europe, thus meeting the goal of being a major contributor to value creation in business and society, training graduates who are competitive in the international job market, and developing new research-based knowledge in partnership with companies and other organizations".

In the Nordic Project, CBS's rationale for participating was: "the sharing of good practices both internally and externally has for quite a long time been of paramount importance to the quality work at CBS" (Omar \& Liuhanen, 2005, p. 52). At CBS, management for quality has been an integral part of the ongoing strategic process since the beginning of the 1990s. For CBS, quality is about creating a culture, and quality work at CBS involves the university as a whole. More specifically, quality:

- $\quad$ is embedded in the CBS mission and strategic focus areas

- has strong support from CBS leadership and management

- is located at and supported by CBS Learning Lab

- involves the university as a whole

- is a continuous, systematic activity

- requires a focus on both quality enhancement and quality assurance 
- has an international orientation

- is stakeholder-related

- uses external quality expertise

CBS has adopted the stakeholder-related definition of the concept of quality while accepting the view that there is no definitive and final definition of quality. CBS has adopted Harvey and Green's (1993) concepts of quality where quality means exceptional, perfection, fitness for purpose, value for money, and transformation. Nygaart and Kristensen (2009, p. 8) discussed "CBS is very much driven by a Scandinavian philosophy of management, characterized by centrifugal power distribution, high employee autonomy, informal decision-making structures, personal network relations, and focus on group norms and values". They stressed that "this is in contrast to management through systems building, rules setting, and key performance indicators". They also pointed out "in a culture with such decentralized structures as ours [CBS], personnel at all levels are responsible for the implementing quality strategies and quality initiatives". Nygaart and Kristensen (2009, p. 7) said "to us quality enhancement is about processes of improvement and not about outcome-based statements only".

\subsubsection{Learning Features to Secure Quality}

According to CBS learning features involve quality assurance activities. The following are practices and examples used for this purpose for each of the five notions of quality.

1. Quality as "exceptional" means CBS's ambition to be among the best institutions in Europe and the quality activities for this purpose are:

- CEMS Benchmarking (1995)

- CRE-Audit (1996), CRE Follow-Up (1998) (now EUA)

- EQUIS Accreditation (1999/2000) and Re-accreditation (2004/2005)

- ESMU Benchmarking Programme (ongoing since 2001)

- Internal Research evaluation with international peers (ongoing since 1994)

- $\quad$ AMBA accreditation of 6 MBA programs (2007)

- National Accreditation of study programs by ACE Denmark (ongoing since 2007)

- OECD project on institutional quality initiatives in teaching (2008-2010)

- AACSB self-evaluation report for Initial Accreditation submitted (2009) 
2. Quality as "perfection" refers to the strategic development as a learning university and the quality activities for this purpose are:

- Staff recruitment

- Staff development (competence development strategies and practices, administrative networks of knowledge sharing)

- Benchmarking (internal and external)

- Quality culture (focus on teaching, learning and research)

- Curriculum development

3. Quality as "fitness for purpose" is related to the CBS's stakeholders and the following quality initiatives striving for increased partnership with the business community:

- Dialogue with the business community

- Dialogue with graduates (alumni)

- Advisory boards

- Employer Panels

- Corporate partners

- External examiners

4. Quality as "value for money" is connected to the enhancement of accountability. For CBS, it is necessary to demonstrate accountability as a part of quality management. Nygaart and Kristensen (2009, p. 14) reported "as a public university, it is important for CBS to demonstrates its accountability and responsibility to one or more external constituencies". In the Nordic Project, (Omar \& Liuhanen, 2005, p. 59) appeared: "At CBS, greater responsiveness to external demand for accountability, transparency, credibility etc. is not seen as an antithesis to self-regulation, but rather as an element of public responsibility, safeguarding autonomy". The following are quality assurance activities for this purpose:

- External accreditation by ACE Denmark (ongoing since 2007)

- Performance indicators (2005-2006) (Ministry)

- Performance agreement (2000-2003) (Ministry)

- Internal evaluations - feedback to students on webpage (ongoing)

- Multiple focus group interviews with employers and alumni regarding drop-out rates, curriculum development, competencies of graduates (ongoing)

- Bi-annual qualitative study of the "learning environment" at CBS (since 2004) 
5. Quality as "transformation" refers to empowerment of CBS students to learn. Nygaart and Kristensen (2009, p. 15) mentioned "our graduates must possess transformative skills". The initiatives taken in regard to quality as transformation are:

- Continuous quality improvement

- Curriculum development with a focus on learning rather than teaching (e.g., the article by Nygaard and Andersen distributed to this workshop)

- Evaluation of transformative learning

- Embedding transferable skills into the academic curriculum

- Benchmarking (internal and external) - transfer of 'good practice'

- Development of research based consultancy at CBS Learning Lab

- Use of an external expert

As a learning university, the study programs at CBS are founded upon an understanding of what learning is and how the learning process takes place. This understanding is realized in practice by a wide range of actual course activities designed to promote the learning of the students.

It is a well-known fact that quality is a journey and once one starts, the quality work never ends. Quality is associated with culture and the philosophy of the total organization. Thus, the CBS philosophy serves at this point as a concluding comment:

- Successful quality enhancement is to be made from an organic, bottomup approach where focus is on key stakeholders

- An organic, bottom-up approach leads to commitment and sense of ownership

- Quality enhancement in multiple parts of the organization requires a well-developed information system

- Key stakeholders and key actors have to be brought together in coordinating the quality enhancement process

- Strategy formulation and implementation constantly need to run throughout the entire organization

Nygaart and Kristensen $(2009$, p. 17) reported that in creating a quality culture in CBS, they work "from bottom-up perspective, rather than top-down". For them, it means "decision-making is decentralized"; they noted "a strong focus on practice, rather than on systems". The authors believed that quality enhancement in CBS "is a long-term investment in people, values and norms, focusing on the creation of a quality culture rather than on a bureaucratic quality management system" (p. 17). 


\subsubsection{Assessment Plan and Strategic Planning Approaches at Indiana University-Purdue University Indianapolis (IUPUI)}

IUPUI is a major urban research and academic health sciences campus, with 22 schools and academic units that grant degrees in over 200 programs from both Indiana and Purdue Universities. Over 30,300 students attend IUPUI. The IUPUI's mission statement, which was approved by the Trustees in November 2005, is as follows:

IUPUI's mission is to advance the State of Indiana and the intellectual growth of its citizens to the highest levels nationally and internationally through research and creative activity, teaching and learning, and civic engagement. By offering a distinctive range of bachelor's, master's, professional, and Ph.D. degrees, IUPUI promotes the educational, cultural, and economic development of central Indiana and beyond through innovative collaborations, external partnerships, and a strong commitment to diversity.

IUPUI's vision is to be one of the best urban universities, recognized locally, nationally, and internationally for its achievements. As recently as August, 2010, IUPUI was ranked as the fifth best up and coming urban university in the US ${ }^{5}$, thus, operationalizing its commitment to excellence and its vision and improving its position from seventh place in 2009.

\subsubsection{Planning and Institutional Improvement}

A key person in IUPUI's planning process is Trudy Banta. Banta's work centered on the key question: "Is assessment itself making a difference?" In the conclusion to her work, the answer was a resounding yes (Banta, 2003, p. 357). She came to this conclusion during her tenure at IUPUI. Trudy Banta pointed out how effective assessment creates an ongoing challenge to higher education institutions at all levels.

IUPUI, in pursuing its mission and vision, demands excellence in the three key areas of every comprehensive university (i.e. Teaching and Learning, Research, and Civic Engagement-Locally, Nationally, and Globally). Each of these three core activities are characterized by:

- Collaboration within and across disciplines and with the community

- Commitment to ensuring diversity

- Pursuit of best practices

5 http://newscenter.iupui.edu/4802/AUPUI-Moves-up-the-List-of-Upandcoming-National-Universities: US News \& World Report. 
The mission of Planning and Institutional Improvement is to develop, integrate, and continuously improve institutional planning, implementation strategies, evaluation, and improvement activities at IUPUI. Planning and Institutional Improvement goals are to work with IUPUI campus and school administrators, faculty, students, and community representatives in order to:

- Clarify, prioritize, and communicate broadly IUPUI's vision, mission, and goals

- Enable all academic and administrative units to coordinate their mission, vision, and goal statements while aligning them with those of the campus in general

- Provide leadership, consultation, and resources to support the evaluation of campus, unit goals, and implementation strategies

- Derive key indicators of institutional effectiveness and provide periodic reports to external constituents

- Derive, prioritize, recommend, and assist in implementing improvements based on the findings evaluated

The components of the Planning and Institutional Improvement Office that facilitate the processes include:

- The office's immediate staff

- the IUPUI Economic Model Office

- the Office of Information Management and Institutional Research

- the Office of Institutional Effectiveness

- $\quad$ the Testing Centre

With respect to improvement, at the Planning and Institutional Improvement website readers can find themes related to the Accelerated Improvement Process, Economic Modeling and Budget Analysis, Placement Testing Service in Support of Academic Advising, and Best Practices. To the stated goals of Best Practices are:

1. to conduct effective planning and improvement processes

2. to provide good stewardship of resources

3. to respond to and manage expectations of stakeholders

4. to provide effective human and physical resources to further the mission of the institution

5. To communicate and manage reputation.

A related process concerns the Accelerated Improvement Process (AIP), which is expected to respond to the question: "how do you know when a process needs improving?"

- it takes too long 
- the work is piling up

- lots of mistakes are occurring

- everyone is stressed

- everyone does it differently

- no one is sure who is responsible

The Accelerated Improvement Process is expected to reduce cycle time, to improve quality processes, and to help to create better programs. Below is an "executive summary" graph regarding the culture of evidence (Figure 2-2). The quality circle for IUPUI incorporates the primary activities of Planning and Budgeting, Implementation, Evaluation, and Improvement based on a culture of evidence that consists of assessable outcomes, instrumentation, tracking-data collection analysis, and application of findings.

Although it may seem paradoxical, given the long planning tradition and the elaborated models available, in fact, however, it reveals healthy insight into the limitations of the administration's influence on faculty, Banta contended:

The Planning and Institutional Improvement Office is still at some distance from our goal of establishing a robust scholarship of teaching, learning, and assessment at IUPUI. That is, faculty activity that can provide students with a strong foundation of knowledge, skills, and values but also can seek direction for continued improvement through the collection of evaluative data and reflection on the implications of the findings for practice. We invite others to travel this road with us, either as evaluators who can make recommendations along the way for improving our program or as participants in a similar approach to general education and assessment on their own campuses.

Banta pointed out that "assessment is an essential component of program development and improvement". Banta highlighted that "comprehensive assessments are used to inform import decisions, especially those to improve curricula and pedagogy but also regarding planning, budgeting and accountability" (Banta, 2006, personal communication). All major programs at IUPUI, including orientation advising and peer mentoring, are regularly scheduled for reviews, a two-year process with self study and outside review components. Improving and providing effectiveness, using assessment results for planning and resource decisions, involving faculty, measuring students learning outcomes using quantitative and qualitative approaches, and linking data into action are major assessment strategies for IUPUI. 


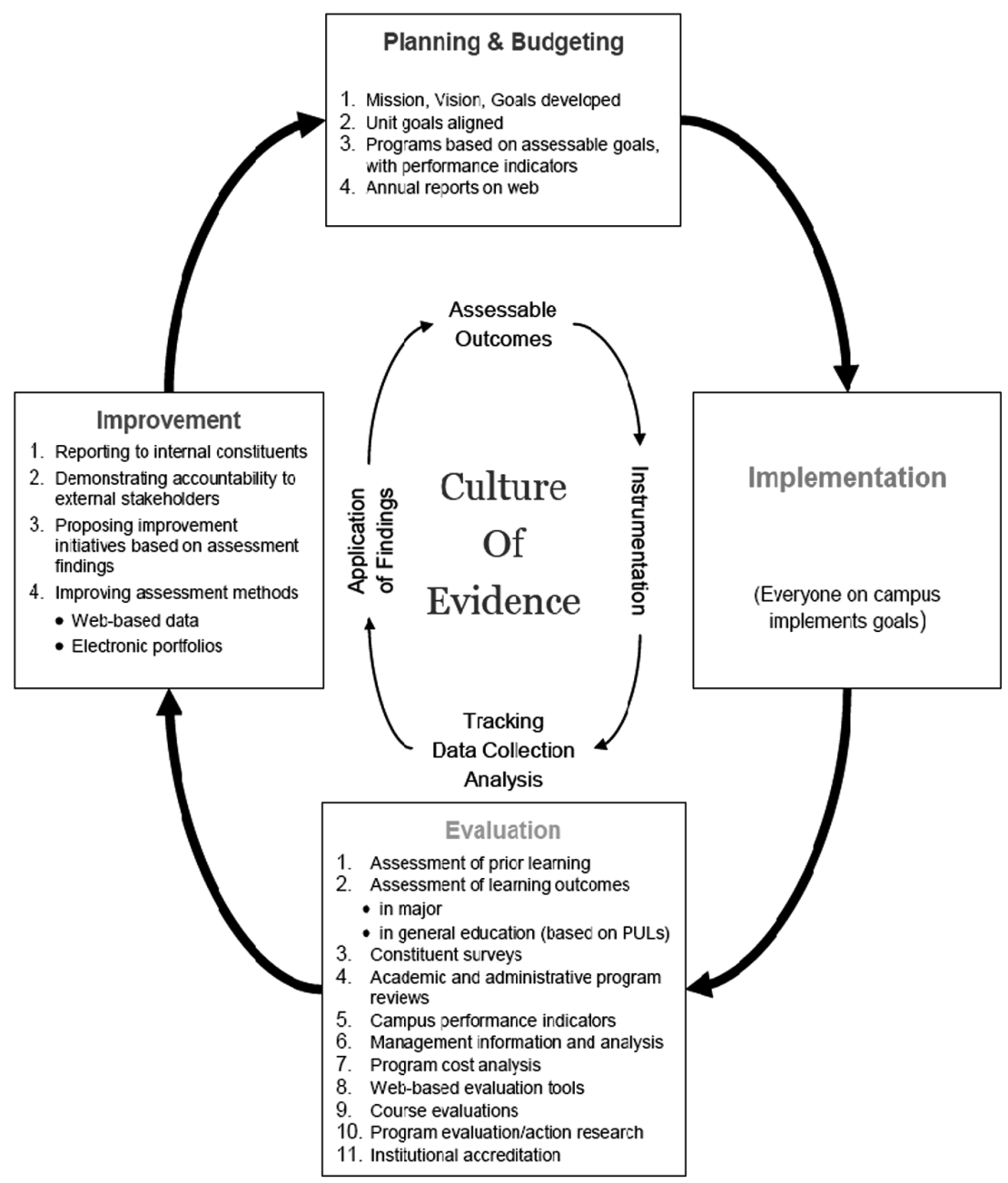

(9) Indiana University-Purdue University Indianapolis

Figure 2-2 Culture of Evidence at IUPUI

Sources: Banta 2006, personal communication, with permission 


\subsection{Concluding Remarks and Lessons Learned}

This chapter's purpose is to explore approaches to quality management practices in the higher education field. The key question is not so much a technical onehow to implement quality management activities-as it is one that seeks to discover the rationale and effects of quality management activities. Accordingly, in the section that discusses quality practices from abroad, "practices" became a blend of several issues reviewed in the first section. Personal communications and site visits were especially important to facilitate a better understanding - from theory to practice.

\section{The WCU Quality Practice}

The quality practice from WCU achieved its goals by focusing on its mission and the strategic Plan for Excellence and by practicing Distributed Leadership-a management philosophy that emphasizes individual expertise, teamwork, and creativity. Distributed Leadership fosters a campus culture that views challenges as opportunities for entrepreneurial expression rather than as a reason for distress. Distributed Leadership is expected to analyze problems and identify limits from varied angles so that risks are reasonable and scarce resources can be allocated in ways that optimize results. The Excellence Plan, in combination with the President's Distributed Leadership, can be seen as a guide for the university in an era of diminishing support and increased demands-most helpful while it is facing accountability problems and issues requiring performance improvement.

The leadership role is crucial to the development of excellence at this university. At WCU the practice I observed was a type of leadership triad as found in the MB: Leadership (Category 1), Strategic Planning (Category 2), and Student, Stakeholder, and Market Focus (Category 3). Senior leaders must set organizational direction and seek future opportunities for their universities. President Adler, without taking an explicit MB approach, included materials for a good recipe regarding continual improvement of her university, incorporating strategic planning that focused on her students' and stakeholders' needs. As a senior leader, President Adler set organizational direction and sought future opportunities for her university.

\section{The CBS Quality Practice}

CBS management's quality practice has been an integral part of the strategic process, especially through their involvement with several quality assurance activities. It is believed by many that quality is a journey, and once that journey has begun, the quality work one must produce is never-ending. Quality is 
associated with the culture and the philosophical essence of the organization under review. In CBS, the quality practice I observed integrated institutional quality with the surrounding culture. In that case, it seemed that quality was not a managerial concept of accountability, but a culturally shaped philosophy underlying its every day practices. It seemed that the quality created within the culture at CBS reflected a bottom-up rather than top-down approach. Furthermore, the majority of the quality practices at CBS were based on the concepts of quality launched by Harvey and Green (1993). From that practice, I observed that creating a quality culture is more closely connected to quality improvement and quality assurance. The quality improvement observed at CBS was about the processes of improvement and not merely about outcomes, although these processes of improvement usually resulted in good outcomes.

\section{The IUPUI's Quality Practice}

The final quality practice in this part is related to IUPUI's assessment plan and institutional improvement. The most prominent lesson learned from IUPUI Planning and Institutional Improvement is that the university, in order to have a better understanding of the consequences of the changes made or being made on its campus, should be viewed in light of its mission and upon its practices on outcomes assessment. How the results of assessment are being used for development or improvement and accountability purposes at the institutional and departmental level is emphasized accordingly in this practice. In the more than 20-year history of assessment at IUPUI, the establishment of an assessment committee was among the first steps taken. The members of this committee had initially decided that the best way to encourage faculty to undertake assessment on their own was to offer them a series of small, competitively awarded grants. A newsletter was issued periodically and mailed to all faculty members to keep them informed of progress made. The IUPUI Assessment Committee was strengthened by its affiliation to a system-wide assessment initiative staffed by a special assistant to the President of the University. The strategy process works as a tool for organizational performance based on ongoing comprehensive assessments. Assessments were considered by Banta as instruments for performance improvement or in other words, a "culture of evidence" involving attention from all the university's stakeholders.

Quality practices in this chapter include also applications of the models of EUAIEP, MB, or ISO. MB winners published their quality journey as requested by the Award, which included sharing best practices; therefore, I decided to investigate additional quality practices. EUA-IEP and ISO, as discussed later in this book, will be empirical case studies. The reason here, as noted, was to see whether the theory influences quality assurance work in actual practice. It seems that by using 
quality management initiatives one could find evidence of their adoption along with identifiable practices (thus, CBS made conscious use of the EUA-IEP). Some universities take into account their mission and vision while implementing their "journey to quality"; however, in a slightly different format.

To summarize, I argue that there are a number of models that could help universities in their journey to performance improvement or even to excellence: I detailed the EUA-IEP, MB and the ISO. To present relationships and differences among these models is a challenging approach, as the models were developed for different types of organizations, in different countries and continents. All of them are related to quality. However, the ISO focused on the delivered quality of separate products or services, while the other two (EUA-IEP and MB) were primarily concerned with overall university performance. Still, all models focused on the importance of leadership, commitment, and involvement of faculty and staff, including management that is based on process, data collection, and analysis. ISO is geared mostly towards products and services (more of a customer-satisfaction oriented approach) and it does not concern the change process. On the other hand, EUA-IEP and MB could be perceived as models to support the learning process, which includes acknowledgement and importance of stakeholders within the organization. Although only MB has an explicit criterion regarding social responsibility, the EUA-IEP universities could also provide evidence as to how they too, serve society.

Another observation can be made in regards to consultancy, because in the ISO certification, consultancy plays a crucial role. A similar trend can be observed in $\mathrm{MB}$; however the EUA-IEP has a different orientation. Rectors who serve on the evaluation team provide specific information for universities to develop their quality and strategic management. This is a key point for attention, because that type of auditing provides trust within the university and all its stakeholders. Having provided professional leadership and by building an atmosphere of mutual trust and inclusiveness within each university, it is no wonder that I failed to find criticisms against EUA-IEP team reviews in CBS.

The EUA-IEP has an improvement orientation and it focuses on the entire university. Additionally, the EUA-IEP offers follow-up activities. This service to its members can be perceived as a continuous process and universities usually embrace it as they engage in improvement of their performance.

Regarding lessons learned, it is worth noting that each model and quality practice from abroad reflected continuous improvement as a significant aspect to achieve excellence. These universities seemed to adopt quality management as a 
normative action, in the sense of increasing its professional approach to the core process of education. Universities became "open" to quality management and it is clear that they are changing in order to respond to their environment. All these models and practices mentioned are based on the ideas of quality management; therefore, those higher education institutions which positively engage in adopting quality initiatives also demonstrate the ability to change.

Quality management suggests a normative idea (behavior), because adoption and implementation of quality management practices require data collection and analysis, assessment, strategic planning, and writing reports; these activities all require professional involvement from multiple sources such as consultants, reviewers, auditors, managerial staff, and contributions from faculty who voluntarily share best practices. Therefore, if one were to gain understanding about this normative process and were to attempt to correlate the influences provided from such a wide variety of sources, which remain unique to each university, it might be possible to ascertain how the adoption process occurs as well as track how interdependent each decision is, because at each level, there remain multiple influences moving into the next step. Consequently, the process here is to identify those individual steps that achieve the broader goal, the isomorphic behavior of adoption and successful implementation of quality management at the university level. But what behavior makes all these universities to respond accordingly? Which environmental factors have the most influence on a university's adoption of quality management? 


\section{Environmental Challenges, Organizational Change and the Adoption of Quality Management: Building the Conceptual Framework}

This chapter introduces the theoretical considerations for our study on the adoption of quality management in Greek universities. This study is rooted in organizational research on management in higher education and quality management as an instance of organizational change. This study provides a contribution to the literature about higher education organizations (for an overview, see Gornitzka et al., 2005; Rhoades, 1992). Moreover, while quality management in Greek higher education is a very recent social phenomenon, it provides new material for the study of higher education organizations. Additionally, this study also contributes to organizational theory in general, including studies about quality management in both the business world and in the field of higher education. Furthermore, this study addresses a gap in the literature as far as quality management in Greek higher education is concerned.

Open systems theory was chosen as a starting point for the theoretical considerations when addressing universities in a changing environment. Thus, the first section in this chapter discusses open systems theory and attempts to firmly establish some basic ideas and perspectives about how the open systems theory is used to describe and explain organizational characteristics and behavior. A discussion follows, which includes what organizational studies have mentioned regarding the specifics of universities' organizational characteristics. In today's competitive climate, higher education institutions worldwide continually feel pressure to change because the world around them is changing. Therefore, there is an elaboration on the meaning of the environment in open systems theory in general as well as a discussion of what organizational studies noted about the specifics of universities' in relation to their environment.

The capacity of the modern university to respond to change has, however, remained an elusive enigma (Johnson et al., 2003). Perhaps, the greatest challenge for the university in the Knowledge Age is determining how to balance its historic traditions and heritage with powerful societal forces for change. Research in higher education thus needs to refine its understanding of "subtle ways in which old and new cognitive schemes compete, collapse and merge" (Maassen \& Stensaker, 2005, p. 215). 
Neo-institutional theory has been one dominant approach in organizational theory for the past 30 years. Neo-institutional theory argues that organizations attempt to incorporate norms from their institutional environments; hence, they can gain legitimacy, resources, stability, and enhanced survival prospects (DiMaggio \& Powell, 1983; Meyer \& Rowan, 1977; Oliver, 1997; Scott, 1987). This perspective has become an important approach for explaining how organizations use rationalized formal structures and corresponding policies to adapt to institutionalized prescriptions drawing from their environments and has been applied to different organizational phenomena (Casile \& Davis-Blake, 2002; Csizmadia, 2006; Erden, 2006; Gornitzka, 1999; Gornitzka \& Maassen, 2000; Kraatz \& Moore, 2002; Leblebici et al., 1991; Lounsbury, 2001; Sherer \& Lee, 2002; Sporn, 1999). Neo-institutional theory has also developed concepts to understand how environmental pressures may increase organizational homogeneity in a certain field (isomorphism) while organizations may nevertheless respond to such pressures in a rather ritualistic, symbolic way. Neo-institutional theory has also been useful in studies on organizational change-and stability-in higher education in general and quality management more specifically. Thus, the second section of this chapter addresses theoretical issues in relation to neoinstitutionalism ${ }^{6}$ in organizational studies and isomorphism more specifically.

Subsequently, the chapter moves on to review empirical contributions as applied to the field of higher education as well as empirical studies of quality management outside the higher education field. The final section draws the theoretical foundations of the study together and subsequently zooms into a conceptual framework to be applied in the empirical analysis and a reformulation of this study's research questions.

\subsection{Universities as Organizations in an Open Systems Perspective}

By design, the present study deals with organizations and their environmental contexts. Scott (1981, p. 22) stressed that "organizations are not closed systems, sealed [off from] their environments, but open to and dependent on flows of personnel and resources from outside their own systems". Organizations, as open systems, exchange ideas with and give feedback to their external environment. Morgan (1998, pp. 40-41) stated that "the systems approach builds on the principle that organizations, like organisms, are 'open' to their environment and must achieve an appropriate relation with that environment if they are to survive". In a similar vein, Scott (2003, p. 91) stated that from an open system

\footnotetext{
6 In the literature the concepts "neo-institutional theory" and "new institutionalism" are used exchangeable.
} 
point of view, "there is a close connection between the condition of the environment and the characteristics of the system within it: a complex system could not maintain its complexity in a simple environment".

Organizational theorists (Katz \& Kahn, 1978; Morgan, 1986, 1998; Scott, 1995) discussed how open systems theory has generated many new concepts of thinking about organizations. Morgan (1998, pp. 42-44) noted that "the open systems principles have been extremely influential and have focused understanding of organization in several ways: Open systems theory emphasizes the importance of the environment in which organizations exist. Organizations are seen as sets of interrelated subsystems. The open systems approach encourages us to establish congruencies or 'alignments' between different systems and to identify and eliminate potential dysfunctions". Harrison (1994, p. 28) provided a version of the open system model building on the work of authors such as Daft (1992), Katz and Kahn (1978), Nadler and Tushman (1989). This version of the model is portrayed in Figure 3-1. The elements of the system are: goals and strategies, culture, behavior and processes, technology, and structure. We also observe the inputs or resources of the systems and their outputs.

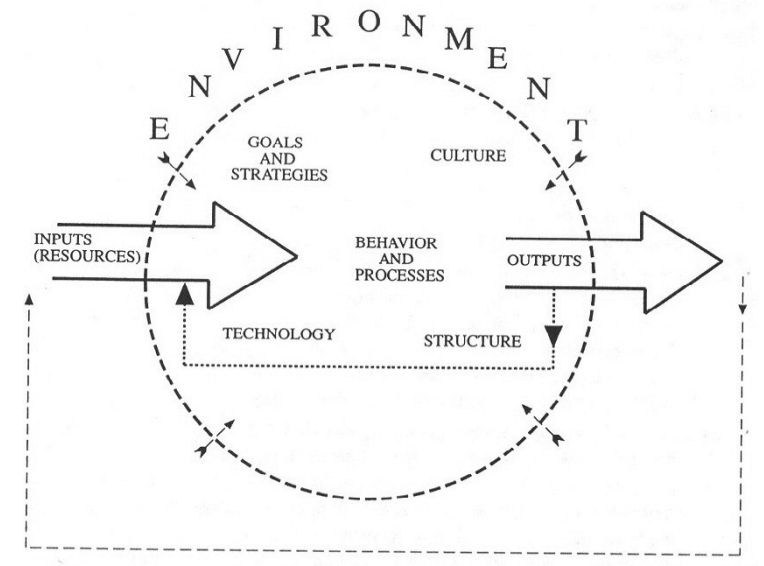

Figure 3-1 Organization as an open system

Source: Harrison (1994)

Mintzberg (1983, pp. 9-10) discussed that organizations can be analyzed through five parts. The operating core which encompasses those members-the operators-who perform the basic work of producing the products and rendering the services. The strategic apex - people who are charged with the overall responsibility for the organization. The middle line managers with formal authority. The technostructure that served the organization by affecting the work of others, and the support staff. Hall (1999, p. 46) pointed out that the 
organizational structure can be considered the arrangement of organizations parts. He emphasized that complexity, formalization, and centralization can vary within a single organization. Additionally, he stated that "complex organizations contain many subparts requiring coordination and control, and the more complex an organization is, coordination and control become more difficult to achieve" (Hall 1999 , p. 52). Additionally, Hall (1999, p. 84) discussed how the structure of an organization falls into two major categories: the context (i.e. size, technology, internal culture, environment and national cultural factors) and the design (i.e. strategic choice and institutional model of structure).

Several scholars discussed elements of open systems theory as it relates to higher education institutions. Birnbaum (1988, p. 47), for example, viewed colleges and universities as open and dynamic systems composed of patterns of interacting elements and subsystems loosely or tightly coupled to each other and to their environment. He also suggested that in order to learn how colleges and universities work, it is necessary to see them as organizations, as systems, and as inventions. His argument brings us to the general applicability of open systems to universities. Certainly, universities have many generic characteristics in common with other organizations. They have their goals, hierarchical systems and structures, officials who carry out specific duties, decision making processes that set institutional policy, and a bureaucratic administration that handles routine business. Much emphasis has, however, been given to the unique nature, purpose and dynamics of universities. Researchers characterized universities as complex organizations with unique characteristics (Baldridge et al., 1977; Baldridge \& Deal, 1983; Birnbaum, 1988; Bolman \& Deal, 2003; Clark, 1983; Csizmadia, 2006; Dill, 1992; Enders, 2004; Gornitzka, 1999; Sporn, 1999). Some distinguishing characteristics that affect a university's adaptation to change are goal ambiguity, task complexity, specific professional and administrative values, and environmental vulnerability. Enders (2004, p. 362) characterized universities, for example, as "multi-purpose or multi-product" organizations and stated that "universities are institutions that, in all societies, have performed basic functions which result from the particular combination of cultural and ideological, social and economic, educational and scientific roles that have been assigned to them". This brings to mind what Kerr (2001, p. 7) observed about the US universities, which are "so many things to so many different people that it must, of necessity, be partially at war with itself".

Weick (1976) analyzed higher education organizations as loosely coupled systems in which vertical and horizontal integration are difficult to achieve and where responses to external pressures for change might not touch upon the technical core of the organization. In such a system, it is possible that quality management practices within one university department may have no impact on another 
department. In a similar vein, Birnbaum (1988, p. 31) pointed out that elements of universities are responsive to each other, but not necessarily directly and predictably. In such a system, synchronization of an entire university is very difficult. Cohen and March (1974) addressed these issues in a similar vein looking at universities as organized anarchies reflecting that universities have ambiguous goals, unclear technology, and ephemeral participants.

Mintzberg (1979) characterized higher education as a professional bureaucracy with professionals in the operating core given considerable control over their own work. Therefore, much power rests at the bottom of the organization in the hands of those professionals. Clark (1997, p. 171) stated that "in a college, the key group $\ldots$ is the senior faculty. When the senior men are hostile to an emerging theme, however it is introduced, its attenuations are ensured". Birnbaum (1988, p. 24) claimed that leaders in universities may have relatively little influence over outcomes compared to other forces that affect the organizational functioning. He argued that while universities have boards and presidents with formal control, they also have faculty and student governing bodies and administrators with varying levels of responsibility and power. It appears that the power configuration in higher education institutions is complex and diffused across many actors.

The argument that an organization does not and cannot exist in a vacuum also implies that organizations interact with their environments to achieve basic objectives (Gornitzka, 1999). "The prevalence of an open-systems approach in organization theory has meant a focus on the relationship between the individual organization and the environment" (Rhoades, 1992, p. 1886). Organizations' external environments include a variety of elements and influences that can affect them including technological, legal, political, economic, demographic, ecological, and cultural elements (Hall, 1999; Pagano \& Verdin, 1997, Scott, 1995).

Hall (1999, p. 207) emphasized that technological conditions "are a starting point, since this topic and the research surrounding it have already been subject of much attention". He mentioned the works of Perrow, Lawrence, and Lorsch, among others and underscored that organizations operating in an uncertain and dynamic technological environment exhibit structures and internal processes different from those operating in a more certain and unchanging technological environment. He argued that technological conditions have implications essential to our understanding of organizational-environmental interactions and advocated the study of service-oriented organizations such as schools, social work agencies, and hospitals, where the same types of technological shifts can be seen. Hall (1999, p. 208) maintained that "organizations do not respond to 
technological change through simple absorption. Instead, the organization's political process operates through the advocacy of change or stability". He observed that "since the rate of technological and all other environmental changes is not constant for all organizations, the degree to which organizations must develop response mechanisms varies" (p. 208).

Several scholars argued that higher education has entered a new era. The notion that radical change is facing today's universities is obvious, and the list of needed changes is widespread. Drucker $(1992$, p. 97) argued for example that:

it is a safe prediction that in the next 50 years, schools and universities [in the USAP] will change more and more drastically than they have since they assumed their present form more than 300 years ago when they organized themselves around the printed book. What will force these changes is, in part, new technology ... in part, the demands, of a knowledge-based society in which organized learning must become a lifelong process for knowledgeable workers; and in part, new theory about [how] human beings learn.

Sporn (1999) described how several environmental changes (restructuring the economy, changing role of the state, shifting demographics, new technologies and increasing globalization) are impacting demands of access, quality, cost, and effectiveness of education at colleges and universities. She emphasized that these new environmental demands triggered internal responses from universities around restructuring, retrenchment, re-engineering, (total) quality management, strategic planning, financial accounting, and technology transferred. Internationalization, globalization, regionalization and de-nationalization are other changes in universities' environment (Enders, 2004).

Such changes in universities' environment may be generic while several authors have convincingly argued that we need to take into account that countries are conspicuously different from each other and that this distinctiveness is reflected in the way that organizations are managed and respond to environmental change (Olie, 1995, in Myloni et al., 2004, p. 61). Myloni et al. (2004) stated that management and organization cannot be isolated from their particular cultural environment. The authors argued that Greece (together with France and Italy) according to Koopman et al. (1999) - belongs to a separate cultural cluster in Europe, as opposed to the North-West. Therefore, it is appropriate to scan at national level environmental factors such as political/egal, European, technological, economic, and sociocultural in order to examine subsequently the adoption of quality management in Greek higher education. Chapter 5 provides such an environmental scanning (Morgan, 1998). 


\subsection{Neo-Institutional Organizational Theory}

The beginning of neo-institutionalism has been marked by the work of Meyer and Rowan in 1977 building on the social constructivist perspective of Berger and Luckmann (1967). Oliver (1997, p. 700) wrote in her review of Berger and Luckmann's work that institutional theory "suggests that institutionalized activities are the result of interrelated processes at the individual, organizational, and inter-organizational level of analysis. At the individual level, managers' norms, habits, and unconscious conformity to traditions account for institutionalized activities". Scott (1987, p. 496) suggested that Zucker (1987) and Meyer and Rowan (1977) advanced the idea of Berger and Luckmann's conceptual framework and applied it to organizational institutions. Powell and DiMaggio (1991, p. 8) summarized that "the new-institutionalism in organization theory and sociology comprises a rejection of rational-actor models, an interest in institutions as independent variables, a turn toward cognitive and cultural explanations, and an interest in properties of supraindividual units of analysis that cannot be reduced to aggregations or direct consequences of individuals' attributes or motives".

Meyer and Rowan (1977) argued that organizations design their formal structures according to the prescriptions of myths in the institutional environment in order to acquire legitimacy which in turn increases their chances of survival. From a neo-institutional view, organizations operate in an environment dominated by rules, requirements, understanding, assumptions, beliefs and procedures (scripts) about what constitutes appropriate or acceptable organizational forms and behavior (Meyer \& Rowan, 1977; Oliver, 1997; Powell \& DiMaggio, 1991; Scott, 1987). Gornitzka (1999, p. 9) pointed out that many of the studies and seminal theoretical contributions within neo-institutional theory emphasize the survival value of organizational conformity to institutional environment. Tolber and Zucker (1983, p. 22), claimed that their findings "provide strong support for the argument that the adoption of a policy or program by an organization is importantly determined by the extent to which the measure is institutionalizedwhether by law or by gradual legitimization". Gornitzka (1999) suggested that conformity often has a ritualistic nature, where organizations construct symbols of compliance to environmental change. To this extent, organizations are seeking to combine conformity to environmental expectations with organizational stability. In this respect, a neo-institutional perspective will emphasize the stability of organizations and the barriers to change that exist within organizations. Most changes in organizations are the result of relatively gradual responses that relate organizations more closely to their environment. Such changes are likely to be found in well-developed institutions with stable values, 
interests, perceptions, and resources. This institutional stability is the foundation for institutional flexibility when faced with externally proposed reform initiatives (March, 1988). In such instances, the focus is on internal institutional rules and requirements that define behaviors and organizational forms.

DiMaggio and Powell (1983) extended this argument to consider why and how organizations' attempts to attain legitimacy led them to become similar. This homogenization process is defined as isomorphism, which is "a constraining process that forces one unit in a population to resemble other units that face the same set of environmental conditions"(DiMaggio \& Powell, 1983, p. 149).

DiMaggio and Powell (1991, p. 65) pointed out that the structure of an organizational field cannot be determined a priori but must rather be defined on the basis of empirical investigation. They also added that the process of institutional definition or "structuration" consists of four parts:

- an increase in the extent of interaction among organizations in the field

- the emergence of sharply defined inter-organizational structures of dominations and patterns of coalition

- $\quad$ an increase in the information load with which organizations in a field must contend

- the development of a mutual awareness among participants in a set of organizations that they are involved in a common enterprise.

DiMaggio and Powell (1983, p. 149) differentiated between two types of isomorphism: competitive and institutional. Competitive isomorphism assumes a system of economic rationality: "The organizations compete not just for resources and customers, but for political power and institutional legitimacy, for social as well economic fitness" (p. 150). The second type of institutional isomorphism is "a useful tool for understanding the politics and ceremony that pervade much modern organizational life" (DiMaggio \& Powell, 1983, p. 150). In order to understand and explain why institutional isomorphic changes occur the authors identified three mechanisms, each with its own antecedents: 1. Coercive, 2. Mimetic, and 3. Normative.

According to DiMaggio and Powell (1983, p. 150) "coercive isomorphism stems from political influence and the problem of legitimacy". This pressure reflects the enforcing and regulative aspects of environmental agents that are sufficiently powerful to impose structural forms or practices on subordinate organizational units. Leicht and Fennel (2008, p. 434) contended that "coercive pressures result from the action of regulatory oversight agencies and major resource providers". 
Additionally, they noted, the "certifications, inspections, and claims to speak for broader unorganized constituencies make coercive pressures in well institutionalized, organizational domain credible" (p. 434). However, Tolbert and Zucker (1983, p. 27) indicated that "legal requirements do not always ensure adoption".

Mimetic isomorphism is associated with standard responses to uncertainty (DiMaggio \& Powell, 1983, p. 150). The process of mimetic structural change is not implemented specifically to gain efficiency. Organizations learn to model other institutions indirectly through turnover and transfers, consulting firms, and trade associations. Another institutional perspective derives from Meyer and Rowan's (1977) ideas. Meyer and Rowan (1977, p. 343) suggested that organizations have a social meaning as they are "deeply ingrained in, and reflect widespread understanding of social reality enforced by public opinion, by the views of important constituents, by knowledge legitimated through the educational system, by social prestige, by the law". Meyer and Rowan (1977) and Zucker (1987) observed that as practices become institutionalized, they become regarded in society as legitimate and are adopted by organizations for legitimacy reasons and not for efficiency, which reflects "ceremonial adoption". Leicht and Fennel (2008, p. 434) stated "mimetic pressures are a consequence of the establishment of taken-for-granted methods of organizing. Once specific organizational practices dominate a specific field, resorting to those practices as the best solution to a problem is simply a matter of borrowing from what others do". Haveman and David (2008, p. 581) specified that "mimetic pressures are ubiquitous but subtle. In the early stages of diffusion, adopters of new structures and practices tend to be those facing technical problems of control and coordination, they hope will be solved by these innovations; later, however, as the innovations become widespread, connections between technical rationality and adoption are attenuated and the causal engine shifts from technical rationality to blind imitation, as innovations become taken for granted as the 'right' way to do things".

The third source of isomorphic change, normative isomorphism, derives from the professionalization of organizational fields. DiMaggio and Powell (1983, p. 152) defined professionalization as "the collective struggle of members of an occupation to define the conditions and methods of their work, to control 'the production of procedures', and to establish a cognitive base of legitimation for their occupational autonomy". Professional networks are important sources of normative pressure in that they provide norms that constrain the behavior of their members. DiMaggio and Powell (1983, p. 152) stated that professional networks "create a pool of almost interchangeable individuals who occupy 
similar positions across a range of organizations and possess a similarity of orientation and disposition that may override variations in tradition and control that might otherwise shape organizational behavior". Empson (2006) reasoned that professionalization is associated with homogenization since the professions are driven by the standardization of knowledge, procedures, and outputs. Recently, Hwang and Powell (2009, p. 275) stated that "participation in professional development and training brings nonprofits into closer contact with their external environment and prevalent organizational practices". According to neo-institutional thinking, isomorphism may, however, be at work without actually changing the core activities of an organization: "when institutional elements are incorporated into an organization, they tend to be 'loosely coupled' with the organization's technical core" (Palmer et al., 2008, p. 744).

Finally, Greenwood and Meyer (2008) discussed a seminal article of DiMaggio and Powell stating that it not only highlighted the concepts of organizational fields and isomorphic change but that is was "actually a third signpost ... in its reference to the link between institutions and the influence of the elite interests. Power and elite control are the backdrop against which the whole play of their ideas takes place" (p. 262). The authors contended, "DiMaggio and Powell explicitly asked us to investigate where new organizational forms come from and whose interest they serve". It is thus "difficult to envisage pressures on dependent organizations without evoking images of power and domination" (Greenwood \& Meyer, 2008, p. 262).

\subsection{Neo-Institutional Theory in Higher Education Studies}

In addition to the above theoretical review, this section focuses on the use of neoinstitutional perspectives in empirical studies undertaken in the field of higher education. Several researchers used notions of taken-for-granted values, norms, and beliefs, of symbolic compliance as a sufficient means for the attainment of legitimacy and survival, and of isomorphism in order to examine organizational change in higher education.

Csizmadia, studying the introduction of quality management in Hungarian higher education, claimed, for example, that "management techniques implemented, i.e. quality improvement programs, may help higher education institutions to manage the impression that outsiders have about them, even if they exist more on paper than in practice" (Csizmadia, 2006, p. 40). His empirical evidence supported the idea that general organizational theories are highly relevant in explaining quality management implementation in higher education 
institutions (p. 226). He suggested that there is a need to study the process of quality management implementation (institutionalization) and not only the outcome. Quality management implementation as an outcome that is observed only over a short time hides many of the dynamic processes that should interest policymakers, experts, and academics. Csizmadia also discussed how various fads, particularly quality mechanisms in higher education, failed and became deinstitutionalized. Furthermore, his study revealed much symbolic compliance. He argued that normative isomorphism was probably introduced through external consultancy and that it played an important role in the institutionalization process as well as in protecting universities' legitimacy. In addition, his study revealed that organizational characteristics, such as leaders' commitment to quality management, institutional reputation, and bureaucratic and political decision-making processes, matter for the organizational responses to quality management in Hungarian higher education as well as the inclusion of external consultants (see also Csizmadia, Enders, \& Westerheijden, 2008).

Erden's (2006) study examined the "interplay between historically rooted diversity within the field of Turkish higher education and the institutional regimes put into effect in the early 1970s and then in the early 1980s" focussing on mimetic and coercive pressures for isomorphism. Erden $(2006$, p. 116) pointed out that:

In the absence of strong coercive mechanisms, and when the legal framework itself has multiplicity within, the historical roots of organizations become more effective in influencing their activities, structures and procedures, creating divergence. On the contrary, in the presence of a strong institutional regime and a unified legal framework, then the field becomes homogenized around the organizational features that are emphasized by the coercive pressures. Organizational histories play a role only in the features that are left 'untouched' by the coercive pressures. As the institutional regime becomes weaker, even when the legal framework keeps its unified character, there is more room for diversity.

For future research he suggested one possible study could be the examination of inter-organizational networks and their effects on the evolution of the Turkish higher education field.

From a neo-institutional perspective, an organization's adoption of policies and programs is constrained by the rules, requirements, and values shared by its members on what constitutes appropriate organizational forms and behavior. Rosa, Tavares, and Amaral (2006) analyzed the opinions of Portuguese university rectors and academics on the quality assessments system and its consequences at the institutional level. They maintained that "[a]cademic values and norms are supposed to be better established in older universities than in newly established 
institutions. Therefore, it is expected that the former will be less open to the implementation of a quality assessment process than the latter" (Rosa et al., 2006, p. 148). Additionally, they claimed that some structures and activities related to quality assessment were more frequently implemented in new universities than in classical ones. Rosa et al. (2006) further discussed how new universities have been more adaptable to the environment than traditional universities, trying to dominate market niches related to local or regional demands in order to escape competition from traditional universities and have been more open to integrating outside stakeholders into governing bodies. This has also been reflected in the finding that rectors from new universities had a more positive view of selfevaluation processes. Organizational characteristics, in this case organizational age and tradition, thus again show some explanatory power in studying organizational change. Also, Rosa et al. (2006) found that rectors paid more attention to internal procedures and services, strategic management, and institutional management structures than to actual improvements in the students learning experience.

Lounsbury (2001) studies how heterogeneity in organizational practices is institutionally shaped in looking at a population of colleges and universities in a U.S., Great Lakes State, which varied in how they staffed authorized recycling programs upon adoption. He claimed that one should look at the connections between institutional pressures and variation in the content of organizational practices by addressing "how inter-organizational dynamics and connections to field-level organizations shape responses to institutional pressures" (Lounsbury, 2001, p. 37).

A study by Casile and Davis-Blake (2002) examined how technical and institutional factors affected the responsiveness of public and private universities to a change in accreditation standards. The authors found that a market niche and ties to an accrediting organization affected the responsiveness of both types of organizations; however, they argued that potential economic gains from accreditation (technical factors) had a greater effect on the responsiveness of private universities. Additionally, diffusion through both social cohesion and structural equivalence (institutional factors) had a greater effect on the responsiveness of public universities. Casile and Davis-Blake (2002) referred to Scott (1995) who had noted that accreditation standards are key aspects of the normative environment as a seal of approval for a professional organization. Casile and Davis-Blake (2002) characterized the accreditation by AACSB as a type of rationalized myth that is likely to confer legitimacy to business schools. Stensaker (2000) similarly remarked that improving quality in higher education institutions will largely be a symbolic activity, initiated to ensure the continued 
funding by gaining societal support. Casile and Davis-Blake (2002, p. 184) noted that individuals in key leadership positions can serve as important sources of diffusion of institutional practices through social cohesion, especially when these individuals move across organizational boundaries. By changing employment or by meeting others in professional settings, they take with them information about institutional practices and can cause organizations to adopt these practices. The authors referred to Kraaz and Moore (2002) which demonstrated how organizational change (deinstitutionalization) is induced by executive migration in American liberal arts colleges. More specifically, Kraaz and Moore (2002) found that liberal arts colleges were significantly more likely to adopt new professional programs if the college president had recently been hired from a school that already had these professionals programs.

The homogenization thesis presented in the work of DiMaggio and Powell (1983) attracted scholars also to explain higher education policies in general and the Bologna Process specifically (Krücken, 2007; Maassen \& Stensaker, 2005; Musselin, 2009; Stensaker, 2004; Watson, 2009). Huisman, Stensaker, and Kehm (2009, p. xv) concluded that "the agenda-setting functions of Bologna can imply symbolic and strategic use of the process as exemplified when different countries use Bologna to implement reforms with a more domestic agenda". Krücken, (2007) examined the implementation of the Bachelor and Master reform in German universities by using neo-institutional theory. He found that "the state as coercive actor seems to be the single most important factor in a process, which is accompanied by the stronger role for accountability and leadership within university organization" (p. 197). In addition, he found that normative pressures were associated mostly with the accreditation agencies rather than other formal organizations in the higher education field. Interestingly, he found that leadership became an important organizational factor for understanding organizational transformation.

\subsection{Neo-Institutional Theory and Quality Management Studies}

Looking at the issue of quality management through a neo-institutional lens has also inspired a number of studies outside the higher education field. Westphal, Gulati, and Shortell (1997, p. 368), for example, tested their hypothesis "when TQM adoption is driven by conformity pressures rather than technical exigencies, firms may realize legitimacy benefits rather than technical performance benefits from adoption" in a study on the implementation of TQM programs and the consequences for organizational efficiency and legitimacy in a sample of over 200 US hospitals. By referring to Crosby (1984), they claimed that TQM emphasizes 
employee empowerment and a major tenet of the TQM philosophy is that continuous improvement will most likely occur with groups of individuals who are provided with not only knowledge, skills, and motivation, but also with the authority to take action. From a neo-institutional perspective "TQM could invoke the socially legitimate goal of improving quality without dictating a well-defined routine for accomplishing it". They discussed TQM, like many administrative innovations, as an integrated management philosophy rather than a clearly defined technology or set of techniques. Following neo-institutional theory they assumed that "the later the date of TQM adoption, the greater the level of conformity to the normative pattern of quality practices introduced by other adopting organizations" (p. 386). Their findings are consistent with the view that early adopters are motivated by the technical efficiency gained from adoption (Tolbert \& Zucker, 1983). Westphal et al. (1997, p. 388) also emphasized that "while early adopters, recognizing greater opportunity for efficiency gains and free from isomorphic pressures, enjoy greater technical benefits from TQM by customizing quality practices to their organization's unique capabilities and needs, later adopters trade organizational efficiency benefits for legitimacy benefits by conforming to isomorphic pressures".

Similarly, empirical studies used neo-institutional theory to explain related issues to ISO 9000 and 14001. Initially, Guler, Guillén, and Macpherson (2000) studied the cross-national diffusion of an organizational practice, the global diffusion of ISO 9000, by adopting a neo-institutional framework. They remarked that their study contributes not only to the empirical literature on the worldwide diffusion of organizational practices, but also to organizational theory "by extending the basic postulates of the new institutionalism to the study of international diffusion and isomorphism. In this view, the agency of powerful organizations such as the state and foreign multinationals generates coercive pressures for diffusion and isomorphism to the extent that potential adopters depend on them for resources. The availability of scientific and technical knowledge provides a normative template that may facilitate diffusion. Finally, mimetic isomorphism in the world occurs to the extent that cohesive ties between countries generate coercive or normative imitation and role equivalent network positions increase learning and competition" (p. 226).

Delmas (2002) investigated the diffusion of environmental management standards (ISO 14001) in Europe and in the United States. He concluded that since ISO 14001 is a voluntary standard, firms will implement it if they believe that the potential transaction costs of acquiring certification will be offset by the perceived benefits that the certification will ultimately provide them. "Governments can act as a coercive force to transform the institutional landscape and act as a coercive 
force to favor the adoption of ISO 14001. Multinationals may also initiate the diffusion of ISO 14001 through mimetic mechanism" (Delmas, 2002, p. 101).

Lastly, Boiral and Roy's (2007) study sheds light on organizational problems and the possible ineffectiveness of ISO 9000 certification depending on their organizational problems integration rationale. Their results demonstrated that the nature and intensity of motivations behind a decision to adopt the ISO 9000 standard, which they characterized as a kind of "rational myth", plays a key role in the success of the implementation process and the emergence of organizational problems arising from certification.

\subsection{Conclusions: Developing a Conceptual Framework and Revisiting the Research Questions}

This section draws together the theoretical considerations into a conceptual framework to be applied in the empirical analysis. In addition, our discussion on isomorphism will be used to revise one of the research questions of this study. Before doing so, it is appropriate to briefly summarize the open systems theory and the neo-institutional perspectives.

Open systems theory has convincingly argued that in order to understand organizational change, one must observe an organization as an open system because organizations do not exist in a vacuum. Open systems theory emphasizes the importance of the environment in which organizations exist. Multiple elements such as political and legal, economic, technological, and sociocultural have elements in an organizational environment may be essential in order to gain a fuller understanding of organizational and environmental interactions. Open systems theory focuses on the inputs, outputs, and transformations of organizations insisting on the importance of the environment, emphasizing its impact on the organization.

Neo-institutional theory, as argued by Scott $(2008$, p. x), can be viewed as a specification of open systems theory. From a neo-institutional point of view, organizations operate in an environment dominated by rules, requirements, understanding, assumptions, beliefs and procedures (scripts) about what constitutes appropriate or acceptable organizational forms and behavior. Organizational response to institutional change can thus best be understood as a search for legitimacy within an institutionalized organizational field. 
Within neo-institutional theory, the concept of isomorphism addresses how organizations adopt innovations, such as quality management practices, in response to environmental pressures for competitiveness and legitimacy. It is argued that homogeneity of organizational form is driven by a process of structuration of an organizational field in which forces are at work that make an organization in a population to resemble other organizations that face the same set of environmental conditions. Three mechanisms can be identified by which isomorphic change occurs: coercive forces which stem from political/egal influence and the striving for legitimacy; mimetic forces resulting from standard responses to uncertainty; and normative forces associated with professionalization, and with shared understanding of "how things should be".

Neo-institutional theory thus contains insights and suggestions that provide a model linking organizational context and intra-organizational dynamics. Neoinstitutional theory is, however, weak in analyzing the internal dynamics of organizational change. As a consequence, it is difficult to understand why some organizations change whereas others do not, despite experiencing the same institutional pressures. During the previous review of empirical studies, I thus identified certain organizational characteristics and processes to be taken into account while studying organizational dynamics, such as mission, vision, leadership, decision-making, age, size, range of studies.

The university has been characterized as a complex organization with many layers, each of which must be studied in order to gather multiple perspectives on organizational change such as the adoption of quality management. Quality management is a multifaceted phenomenon in its own right, and in light of this complexity, binary distinctions become problematic: the university can be the subject as well as an object; national systems of higher education can act as an agent of change as well as the structure that is changed; processes and pressures can be exogenous, yet at the same time endogenous; and actors respond to both. In addition, renewed attention has been given to the power dimension and political consequences of institutional change that has only begun to recapture serious attention when the dominant isomorphic reading started to come under serious attack.

In any case, it is important to investigate the social phenomenon at stake not only at one level but in a multi-level perspective (i.e. macro: higher education system, meso: individual universities, and micro: academic departments, laboratories, and academic support services). Figure 3-2 illustrates the resulting conceptual framework for examining how coercive, mimetic, and normative isomorphism arises from the external environment and how it interacts with university 
characteristics to influence the adoption (or lack of adoption) of quality management in Greek universities.

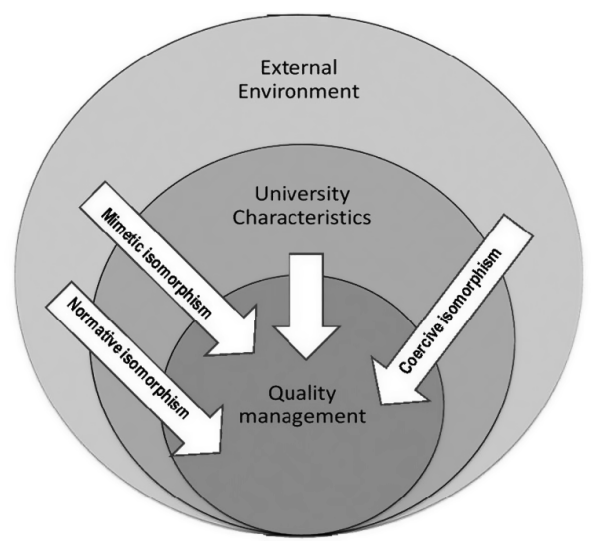

Figure 3-2 Conceptual Framework

As a consequence of the conceptual refinements achieved in this chapter, the third research question must be partially reformulated in order to make it more amenable to empirical research in empirical part of this book:

3. Which organizational factors for stability and/or change concerning the adoption of quality management can we find empirically in Greek higher education? Do these isomorphic pressures (coercive, normative, and mimetic) differ at macro (higher education system), meso (individual universities) and micro levels (academic departments, laboratories, and academic support services)?

Since the improvement of quality is a crucial issue in the field of higher education worldwide, many universities are turning to quality management initiatives and standard strategies intended to improve their activities and results, and thereby improve their institutional quality and competitive capabilities. However, not all institutions have responded to pressures for quality improvement by adopting quality management. In Greece, the focus country of this study, only a minority of public universities until 2006 showed activity in quality management. The slow response by Greek higher education to quality management presents an enigma; despite clear external pressures for quality improvement. The lack of uniformity in the response of Greek institutions has not been adequately explained or understood: some are clearly more active than most others. This study seeks to 
address that knowledge gap by opening up the black box of the dynamics influencing the adoption of quality management within Greek public universities. 


\section{Operationalization}

The purpose of this chapter is to describe the proposed independent and dependent variables and the research methodology necessary to determine the relationship between organizational change factors and quality management practices in Greek public universities. The chapter begins by outlining the key variables and their operationalization. The next section introduces the methodological considerations in relation to the mixed methods strategy used in this study and the reasons for selecting this strategy to gather and analyze the data. The third section provides the specific qualitative and quantitative approaches for the research: surveys and content analysis. The final section addresses ways and criteria for evaluating the quality of the entire study.

\subsection{Variables Presentation and Dimensions}

The first task in preparing to test the conceptual framework outlined in chapter 3 is to identify and operationalize the independent and dependent variables. In other words, the operationalization of the variables that were identified through analysis of the literature outlined in chapters 2 and 3 has to be taken into consideration. The mixed method approach utilized for this study will allow for different ways of operationalization of some variables, so that triangulation of findings will be possible. The two types of understanding, quantitative and qualitative, may frame the adoption of quality management better in Greek higher education. In order to provide the initial input into data analysis, I divided the following section into independent and dependent variables. To study organizational change (introduction of quality management) in the context that influences it, the current study was divided into three levels (macro, meso, and micro) for a more detailed understanding of the adoption of quality management and most importantly, to listen to voices from different target groups. Information and inference from one level became helpful for the next level in terms of revealing the problems as well as explaining the overall phenomenon (adoption of quality management). It was essential for the completion of the project to have a clear rationale for the use of each individual level. The macro level related to the Greek higher education system, the meso level related to individual universities, and the micro level related to academic departments, laboratories, and academic support services. 


\subsubsection{Independent Variables}

The following elements elaborate on each of the three independent variables, namely: external environmental elements, isomorphic pressures, and university characteristics.

\subsubsection{External Environmental Elements}

The first independent variable is made up of the external environmental elements that play crucial roles in an organization's life. As mentioned earlier, understanding an organization as an open system theory proved to be a fruitful approach. Open systems theory emphasizes the importance of the environment in which organizations exist (Morgan, 1998). Additionally, a distinguishing aspect of neo-institutional theory is the argument that organizations are structured by phenomena in their institutional environment and gradually become isomorphic with them (Meyer \& Rowan, 1977). The conceptual discussion above led me to identify five dimensions of the external environment: 1) legal/political, 2) economic, 3) technological, 4) European, and 5) sociocultural.

\section{Legal/Political}

The first sub-dimension or set of indicators focuses on the legal/political aspects of the external environment. Legal issues, sets of rules, and regulations are the main sources for this indicator. In practice, the above items translate to the existing political pressure, such as legal documents in relation to quality management (quality assurance) practices adopted by Greek universities up until 20067. National reports and governmental documents are the appropriate resources to answer the question, "Which legal/political pressures to adopt quality management practices did Greek universities face?"

Within this category, the second sub-dimension consists of the broader socialpolitical trend in views on quality management in higher education. For this second sub-dimension, input from the media (discussed in chapter 6), such as Greek national newspapers, can be used to answer the question, "How much pressure from media was there during the 2005-2006 period regarding the adoption of quality management issues in Greece at the macro level?"

\footnotetext{
7 This study examines adoption of quality management at macro, meso, and micro levels in Greek universities up to 2006. The reason for drawing this border was that in 2007, the context changed considerably when a new law was adopted regarding quality assurance in higher education, and consequently the national system for quality assurance in higher education made its first public appearance.
} 


\section{Economic}

The second dimension focuses on the economic pressure in relation to adoption of quality management practices. In Europe, state budgets for higher education are under scrutiny. At the same time, reform processes aim to make universities financially more independent from the state pushing them to raise additional funds from student fees and from the private sector. At the same time, Gumport and Sporn (1999) pointed out the critical need for efficient management structures and skills for fundraising and development. In order to study these factors, I will approach the following questions: "Who is or are funding Greek universities?", and "Are funding sources related to adoption of quality management practices?" To determine this dimension, appropriate laws regarding funding issues are cited and comments from EUA-IEP evaluators are reported.

\section{European}

The third set of indicators focuses on European dimensions of the external environment. European harmonizations are sets of special regulatory pressures on quality standards, such as the Bologna Process, ISO, EFQM, EMAS, or other international models. Mostly after the Bologna Declaration in 1999, quality assurance and quality management became crucial elements of each country's university structure. To understand the consequences better and to track the development of these evaluation activities in Greek public universities, I will try to define and analyze some specific elements of the quality management practices to examine how they were considered by external auditors (EUA-IEP reports). To study these factors, I will approach the following questions: "How do Greek universities understand European pressure?" and "Do European factors affect Greek universities, and if so, to what degree and at which level (macro, meso, and micro)?" To determine indicators in this dimension, appropriate documents and national reports regarding quality assurance issues will be cited and comments from EUA-IEP evaluators reported.

\section{Technological}

The fourth set of indicators focuses on the technological elements of the external environment and their relation to the adoption of quality management in Greek higher education. While higher education is not in a major sense relying on technology 'hardware' for its processes, management tools can be regarded as technologies in themselves. In this respect, Sporn (1999) pointed out that that sophisticated information systems enable academic organizations to learn about internal processes, marketing, and customers. Their feedback can be used for program development, marketing, and decision-making. Green and Gilbert (1995) point out that "management by information" can make structures and processes more transparent and help increase efficiency and effectiveness. To study these 
issues, I included relevant literature concerning the Greek technological environment in relation to quality management practices.

\section{Sociocultural}

The fifth dimension focuses on the sociocultural pressure in relation to adoption of quality management practices. This dimension includes student populations and their parents, international students, stakeholders, and general social actors in Greek higher education and their special pressures on quality standards or adoption of quality management practices by universities. The study on the role of the media, in chapter 5, and relevant published work are the appropriate resources to answer the question, "Which sociocultural pressures do Greek universities face in order to adopt quality management practices?"

\subsubsection{Isomorphic Pressures}

DiMaggio and Powell (1983) identified three types of pressures that had an isomorphic effect on the members of an organizational field. As previously mentioned in the theoretical section, these pressures are coercive, mimetic, and normative; each one has its own logic and modus operandi. What is common among them is that they make an organization conform to the expectations of its environment.

\section{Coercive pressure}

As indicated previously, coercive pressure reflects the enforcing and regulative aspects of environmental agents that are sufficiently powerful to impose structural forms or practices on subordinate organizational units. Legal issues, sets of rules, and regulations are the main sources for this type of pressure. Greek universities are legal entities and their main source of funding is the state; thus, the funding process is related to coercive isomorphism as well. To that extent, economic factors from the external environment are part of this type of pressure. In practice, the above complex of factors translated to the existing political and economic pressures such as legal documents relating to adoption of quality management practices by Greek universities up until 2006, while funding rules determined the allocation of governmental budgets to higher education institutions. In practice, coercive pressure in my study is equivalent to political and state-related economic environmental elements.

\section{Mimetic pressure}

Mimetic isomorphism is associated with standard responses to uncertainty. In other words, organizations copy the publicly known best practices from other organizations or university units. Thus, a mimetic process occurs when an 
academic organization/university models itself after other institutions that seem similar. In order to measure mimetic pressure that universities feel at all levels (macro, meso, and micro) to adopt quality management practices, I will use the data collected through surveys and gathered perceptions from university leaders, department heads, directors, and university and state documents.

\section{Normative pressure}

Normative isomorphism is associated with professionalization. The literature reveals that professionalization translates to increasing amounts of personnel involved in management of the university with formal education in management and who are involved in occupational socialization. DiMaggio and Powell (1983, p. 152) noted that occupational socialization includes involvement in trade associations, professional associations, consultant arrangements or networks between employers and professional schools. In order to operationalize the normative pressure that universities feel at macro, meso, and micro levels to adopt quality management practices, I will again use the above-mentioned surveys and university and state documents.

\subsubsection{University Characteristics}

University characteristics are the third set of independent variables in this study. Universities, as previously mentioned, are described as organizations with unique characteristics. In the case of the Greek universities, due to their public status, some characteristics such as their mission and decision making processes are equal in all 21 cases, and thus cannot be used as variables. In terms of operationalization, the key variables of university characteristics fall into six categories: vision, leadership, age, size, location, and range of studies. These elements might affect changes such as adoption of quality management. To verify these dimensions I have used appropriate university documents and data collected throughout the empirical studies and observations.

\section{Vision}

Vision represents a definition of success in shaping an organization's future development. As noted above, Lewis and Smith's (1994, p. 194) vision refers to the long-term desired state of an organization, usually expressed in a five to ten year time frame. The strategic goals that the organization needs to focus on in order to succeed are often included in the vision statement, which should inspire and motivate. In practice, this dimension can be derived from a university's internal documents and its website. 


\section{Leadership}

Leadership was defined in chapter 2 as "the reciprocal process of mobilizing, by persons with certain motives and values, various economic, political, and other resources, in context of competition and conflict, in order to realize goals independently or mutually held by both leaders and followers" (Burns, 1978, p. 425). The Leadership Category and the leadership triad from the Malcolm Baldrige quality award questionnaire $(\mathrm{MB})$, which will be treated in-depth in chapter 8, examines how universities' senior leaders address their organizational values, directions, and performance expectations, as well as a focus on students and stakeholders, student learning, faculty and staff empowerment, innovation, and organizational learning. Also examined were university's governance and how a university addressed its public and community responsibilities. In practice, Greek rectors' perspectives, as evidenced through the questions of the leadership category, are to shed light on this element. Document analysis in chapter 7 will also inform this complex and crucial dimension.

\section{Age}

Operationalization of a university's age in most of the higher education studies refers to the longevity of the organization by using two categories, either old (established before 1982) or new.

\section{Size}

The number of students enrolled in universities determines the size of the organization. Small (up to 1,000 students), medium, and large (over 3,000 students) became the categories for this particular characteristic.

\section{Location}

Geographical diffusion is another factor. Organizations can perform their functions at multiple locations (Hall 1999). In higher education, universities are referred as urban (located in the main cities Athens or Thessaloniki) or peripheral institutions located in smaller towns and on islands.

\section{Range of Studies}

I distinguish eight disciplinary fields, i.e. 1. Sciences, 2. Engineering, 3. Medicine and Life Sciences, 4. Law; 5. Behavioral Sciences; 6. Social Sciences and Economics; 7. Humanities, and 8. Arts. These fields have been previously defined in several higher education studies. Universities with programs in more than one of these fields could be characterized as multidisciplinary, meanwhile, the others are monothematic. 
The preceding four university characteristics will return in chapter 5 , where they are presented with appropriate documentation.

\subsubsection{Dependent Variable}

For the purpose of this study, quality management practices are defined as all activities that contribute to defining, designing, assessing, monitoring, and improving the quality of the education in a university. Organizing for quality refers to the description of responsibilities, coordination, and implementation of quality management in the organization. Quality management practices require some kind of structure. In this context, structure reflects the relative positions and authorities of unit/actors in a system. Many factors affect an educational organization's ability to institutionalize a change. The key or essential elements are grouped in seven categories: formalization, centralization, complexity, resources, aims, scope, and modes. These categories are briefly described in the next paragraphs.

Formalization pertains to all the written documentation in an organization as well as referring to established rules, procedures, job descriptions, regulations, mission statements, and policy manuals. All of these elements prescribe acceptable or expected actions by the employees for the purpose of limiting their function and activities within the institution. To identify the pattern of formalization that measures the extent to which rules, procedures, instructions, and communication are written, I will address the following question: "To what extent is the quality management process a formalized and distinctly recognized function in Greek universities?" Written rules and documents regarding this indicator include policy formation as well as internal documents about adoption of ENQA quality assurance criteria, the ECTS handbook, strategic planning reports, organizational charts, and other internal university regulations. To measure this dimension, I collected data through surveys as well as document collection and analysis (Chapters, 5, 7, 8, 9, \& 10).

Centralization refers to the hierarchical level that has authority to make a decision. When decision-making is located at the level of the university as a whole rather than at e.g. the level of departments or faculties, the organization is centralized. This dimension, as pointed out earlier, is similar to committee use as suggested by Greening and Gray (1994) and Koufteros and Vonderembse (1998, p. 2865). To study this dimension, I will address the following question: "At which organizational level-if any-is the organization, strategy and policy of quality management decided in Greek universities?" To measure this dimension I collected data through document analysis (Chapters 5, 7, 8, 9, \& 10). 
Complexity refers to the condition of being composed of many, usually interrelated parts. This dimension has two potential sources of complexity: horizontal differentiation and vertical differentiation. Regarding horizontal differentiation, I identified the number of units or persons involved in quality management. For vertical differentiation, I identified numbers of hierarchical levels involved in quality management. Appropriate resources to investigate this dimension are document analysis in chapter 7 and the surveys in chapters 8,9 , and 10 .

Quality management systems require human and financial resources. It is necessary to take into account monetary allocations (budgets) for this particular process, as well as to identify the number of staff hours. Appropriate resources for this dimension are university documents, document analysis especially from EUA-IEP reports, mentioned in chapter 7 and the surveys in chapters 8, 9, and 10.

Other elements of quality management which were derived from the review of the literature in chapter 2, such as the aim, scope, and models for this core variable, are expected to be defined through surveys, observations, and document analysis. Quality management's aim consists mainly of two elements, as I concluded in chapter 2: accountability and improvement. Van Bruggen et al. (1998, p. 155) contended "the elements that support improvement are: stress on self-assessment, peer-evaluation, operational recommendations, no direct link of financial consequences to evaluation results"; while the elements that support accountability are "public reports, independent experts, meta-evaluation and a follow-up by the government or a governmental agency". In this study, adoption of quality management practices for the accountability issue is related to an "external view of quality assurance schemes", while adoption of quality management for improvement is associated with "institutional factors, academic perspectives", and quality management follow-up activities.

Quality management's scope refers to teaching and learning, research, academic support services, and service to society. Kristoffersen et al. (1998, p. 14) noted: "Theoretically, an evaluation could take into account all aspects of an institution and evaluate all its units, degree granting programmes and activities; alternatively and more pragmatically, it usually focuses on some aspects of an institution - carefully selected to allow for meaningful conclusions" (p. 14). I shall gather information on the scope of quality management from documents and surveys in chapters $7,8,9$, and 10 . 
Quality management models refer to written models or prescriptions such as the Frame-Law 1268/82, EUA-IEP, ISO, EFQM, TQM, EFMD, and other schemes that might be used in Greek higher education and derived from documents and surveys in chapters $7,8,9$, and 10 .

\subsection{Research Methods}

A mixed methods strategy appears to be the most appropriate methodology for this study given the purpose of the research, the research questions, and the conditions in which this study took place. First, the introduction of quality management in a higher education system is a complex issue that may look different at each of the different levels. Second, the core theoretical approach of neo-institutionalism contributes to realizing that need, because it allows for distinguishing different isomorphic processes, as detailed above. The combination of these two factors means that different research methods may be best suited to the different parts of the study, which is precisely the complexity with which mixed methods strategies are designed to cope.

Another reason guided my choice in this matter. The debate that took place in Greece during the period of this study made it difficult to examine quality management directly. Studying a 'hot topic' is already a sensitive and difficult matter in the best of circumstances. However, Greece has an environment that is internationally notorious for its difficulty for conducting empirical social science research: very low levels of cooperation have to be expected (Bourantas et al., 1990; Bourantas \& Papadakis, 1996; Elefteriou \& Robertson, 1999; Makridakis et al., 1997; Psychogios \& Priporas, 2007; Spanos et al., 2002). An exception here would be empirical cases/surveys related to ISO, which reported high response rates: 57 percent by Gotzamani and Tsiotras (2001); 67 percent by Lipovatz et al. (1999). However, that is an exception and the Greek expectation of low responsiveness to research necessitated a sophisticated approach to the empirical part of the study.

The popularity of the mixed methods research (also known as mixed research, multi-method, blended research, multiple methods, or triangulated studies) has being growing over time (Johnson et al., 2007). Mixed methods research has become the most common term to describe this movement (Johnson et al., 2007). The literature on research methods describes mixed methods as a synthesis that includes ideas from quantitative and qualitative research. Researchers stated that mixed methods research is often the best way to address the complex research questions in which they are currently interested (Plano Clark, 2005). Mixed 
methods research is characterized as "an emerging methodology" by Creswell and Plano Clark (2007) who pointed out that this method appears to reflect an opening for many quantitative researchers to use qualitative data. In addition, Currall and Towler's (2003) review suggested that when organizational and management researchers used a combination of qualitative and quantitative methods to investigate organizational phenomena, their research yielded greater information than could be achieved through a single method. The authors pointed out that mixed methods are considered appropriate when research questions concern process and dynamic phenomena such as innovation and change. Greene, Caracelli, and Graham (1989), Tashakkori and Teddlie (1998), as well as Currall and Towler (2003) contended that when qualitative and quantitative methods are used in combination, they harmonize with each other and allow for a more complete analysis. Greene et al. (1989) reviewed fifty-seven method studies to identify five purposes for adopting mixed methods design strategies: triangulation, complementarity, development, initiation, and expansion. Furthermore, Creswell (2002) argued that mixed methods is a strategy for collecting, analyzing, and "mixing" both quantitative and qualitative data at some stage of the research process within a single study in order to understand a research problem more completely.

Therefore, my study of the Greek higher education environment employed several research techniques and data collection methods in order to move as close as possible to the "hot issue" (Johnson \& Ownwuegbuzie, 2004; Ownwuegbuzie \& Teddlie, 2003; Tashakkori \& Creswell, 2007; Tashakkori \& Teddlie, 1998, 2003; Wang et al., 2007). To understand the research methodology used in the current study better, I presented this section in two parts. In the first, I briefly reviewed methodological issues in mixed methods; while in the second, I concentrated on the present study's research design.

\subsubsection{Mixed Methods Methodological Considerations}

A recent definition of mixed methods was provided by Tashakkori and Creswell (2007, p. 4): 'Mixed methods research is a research in which the investigator collects and analyzes data, integrates the findings, and draws inferences using both qualitative and quantitative approaches or methods in a single study or program of inquiry". Several authors (Creswell et al., 2003; Creswell \& Plano Clark, 2007; Johnson et al., 2007; Tashakkori \& Teddlie, 2003) advocated the advantages of mixed methods research designs. At the same time, the same authors underlined the challenges that mixed methods researchers have to face in using this strategy. These challenges include: it requires familiarity with qualitative and quantitative procedures, leads to very extensive and time- 
consuming data collection, and it needs special strategies of analyzing both quantitative and qualitative data. These challenges are not insurmountable, however.

To extend the discussion regarding the mixed methods research strategy, Creswell et al. (2003, p. 223) pointed out that mixed methods researchers can make decisions about four factors-implementation, priority, integration, and theoretical perspective-to select a particular research strategy. Implementation refers either to qualitative or quantitative data collection and analysis in phases (sequentially) or concurrently (data gathered at the same time). Priority refers to whether greater weight is given to the qualitative or quantitative approach. A priority for one type of data or the other depends on the interests of the researcher and what he seeks to emphasize. Integration refers to when the researcher "mixes" the data and is the phase in the research process where the connecting or mixing of the qualitative and quantitative data occurs. Going into more detail about the implementation and priority decisions, Ownwuegbuzie and Teddlie, (2003) recommended that researchers make a series of decisions prior to undertaking mixed method analysis. These decisions underlie the choices of specific approaches, techniques, and interpretive frames used to collect and analyze data from multiple sources. Ownwuegbuzie and Teddlie (2003) also suggested a model for the mixed methods data analysis or integration process. The model includes seven stages: data reduction, data display, data transformation, data correlation, data consolidation, data comparison, and data integration (p. 373).

Teddlie and Tashakkori (2006, p. 24), taking a more holistic approach to the research design, argued that it is impossible to create a complete taxonomy of mixed methods designs and provided a seven-step process for selecting the most appropriate mixed methods design for a research study. The seven steps, which I used to guide the development of my research, are:

1. The researcher must first determine if her research questions require a mono-method or mixed method design.

2. The researcher should be aware that there are a number of typologies of mixed methods research designs and should know how to access details regarding them.

3. The researcher wants to select the best mixed method research design for her particular study and assumes that one of the published typologies includes the right design for her project.

4. Typologies may be differentiated by the criteria that are used to distinguish among the research designs within them, and the researcher needs to know these criteria. 
5. These criteria should be listed by the researcher, who may then select the criteria that are most important to her for the particular study she is designing.

6. The researcher then applies the selected criteria to potential designs, ultimately selecting the best research design for her study.

7. In some cases, the researcher may have to develop a new mixed methods design, because no one best design exists for her research project.

The latter situation applied to this study, and more details are presented in the following section.

Effective integration is another point for consideration, and researchers need to take into account this challenging issue in their projects to which we will return in section 4.4. Teddlie and Tashakkori (2009) pointed out that the most important step in any mixed method study is when the results (findings, conclusions) from the study's qualitative (QUAL) and quantitative (QUAN) strands are incorporated into a coherent conceptual framework that provides an effective answer to the research question. Following them and many other mixed methods researchers, I utilized Morse's (2003, p. 198) notations and abbreviations in this study (Table 4-1).

Table 4-1 Morse's (2003) notations and abbreviations concerning mixed methods

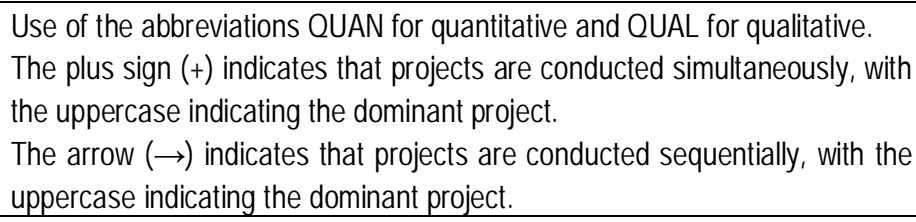

\subsubsection{Present Study's Design}

Teddlie and Tashakkori (2009) reiterated that although typologies of mixed methods designs are "valuable, researchers should not expect them to be exhaustive" (p. 139). The authors proposed that "multilevel designs are possible only in hierarchical organized social institutions, such as schools and hospitals, in which one level of analysis is nested with another" (p. 146). Also, the authors pointed out that "the most dynamic and innovative of the mixed-methods designs are mixed across stages" (p. 146). Tashakkori and Teddlie (2008, p. 102) stated "the quality of a mixed methods study is directly dependent on the purpose for which the mixing approaches was deemed necessary in that study". The authors presented reasons for using mixed methods (i.e. complementarity, 
completeness, developmental, expansion, corroboration/confirmation, compassion, and diversity) based on several sources (i.e. Greene et al., 1989; Patton, 2002; Rossman \& Wilson, 1985; Tashakkori \& Teddlie, 2003). I can characterize the aim for using mixed methods in this study as completeness: "Mixed methods designs are utilized in order to make sure a complete picture of the phenomenon is obtained. The full picture is more meaningful than each of the components [individually]" (Tashakkori \& Teddlie, 2008, p. 102). At the same time, different methods were used for corroboration/confirmation. Tashakkori and Teddlie (2008, p. 103) stated: "unexpected or ambiguous results from a quantitative study might necessitate the collection and analysis of in-depth qualitative data in a new strand of the study". In my project, I studied the meso level by using documents analysis to investigate adoption of quality management and isomorphism. At the same time, a survey study was employed for triangulation purposes. Here, two studies aimed to fully identify and explain the adoption of quality management at the university level by confronting and confirming data from documents and leaders' experiences and perceptions.

My research design thus involved mixing qualitative and quantitative approaches at three levels (macro, meso, and micro) for the purpose of completeness. Like a scaffold, each empirical study of this research was built on and was designed to harmonize with others. The empirical study took place over a two-year period from 2005 to 2007. However, each empirical study occurred at a specific period. The visual model of the procedures for the multi-level mixed design of this study is depicted in Figure 4-1. The following are considerations to illustrate the design model of the study.

The macro level was understood to include issues related to the elements of the external environment in which Greek universities operate. As a preliminary to the actual empirical studies, this book contains a 'quick scan' of the environment derived from documents and observations (chapter 5). The first empirical study (chapter 6) was directed at how the printed media addressed quality management in higher education during a peak period of the debate in 2005. This included a quantitative and qualitative (QUAN+QUAL) content analysis with mixed analysis of data for this particular study's inference. The study was based on content analysis of newspaper inputs from May 2005. This analysis considered whether articles in newspapers were for or against the introduction of quality assurance and which reasons were given for the stated points of view, interpreted in terms of coercive, mimetic, or normative pressure.

Results from the 'scan' of the external environment (chapter 5) and the media study in Chapter 6 were intended as the "cultural knowledge" to give fuller 
understanding of the study's observed phenomenon and they became a valuable asset in the process of making inferences (Tashakkori \& Teddlie, 1998). Thus, the macro level "reflects the researcher's prior understanding of a phenomenon under study" and provided answers to the research question (macro level). In terms of Teddlie and Tashakkori's (2009) definition of purposive sampling techniques, this study used "sampling of [a] politically important case", which "is a special or unique case that involves selecting politically significant or sensitive cases for study" (p. 175).

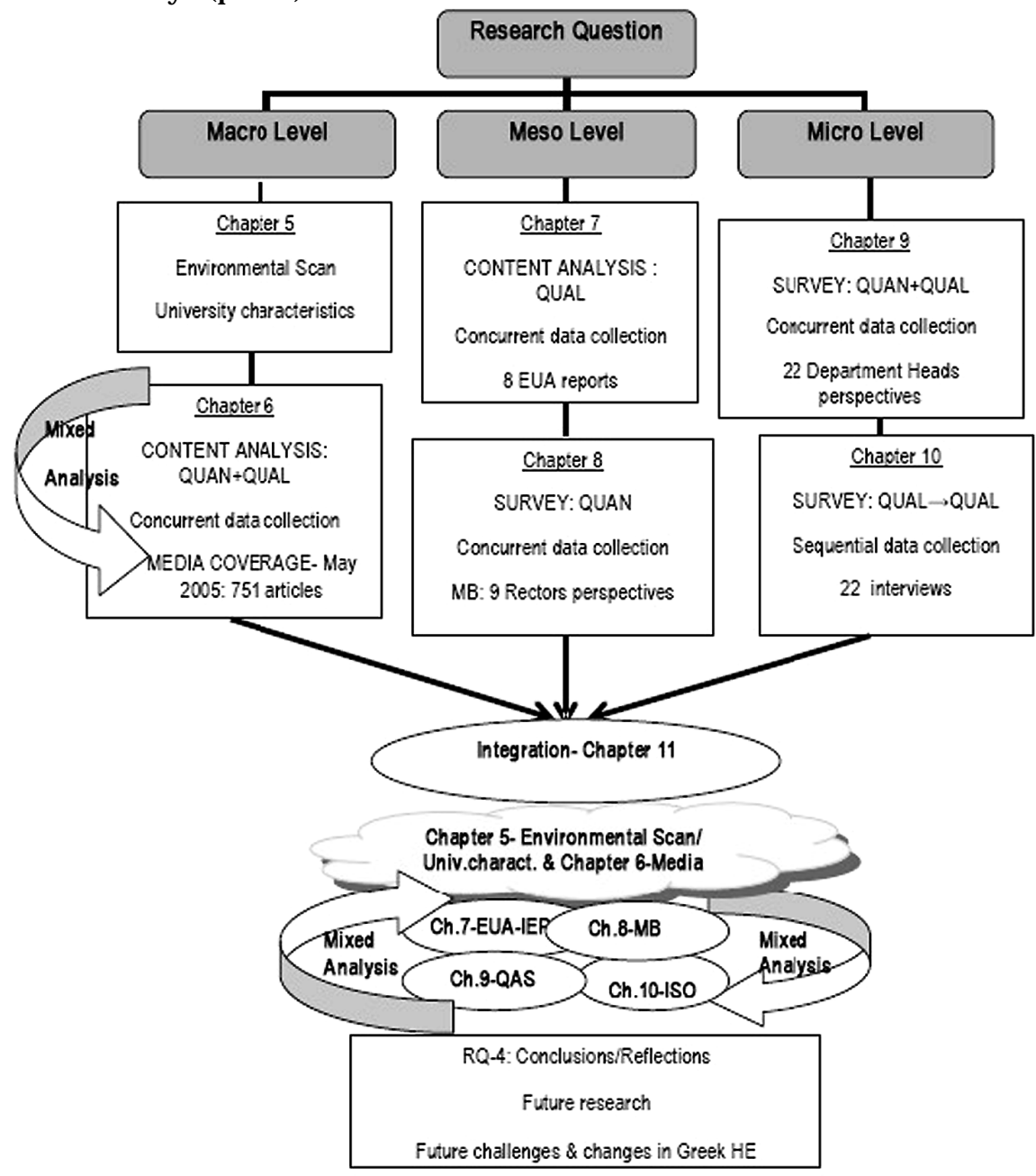

Figure 4-1 Visual model of the procedures for the multi-level mixed design 
The first empirical study at the meso level was a (QUAL) content analysis where the main data were derived from the eight EUA-IEP evaluators' reports about Greek universities (chapter 7). The EUA-IEP was perceived as a key event concerning isomorphic pressures and adoption of quality management within universities in Greece. Fetterman (1998, p. 100) noted that "key events are extraordinary useful for analysis", because they shed light on mechanisms and processes that otherwise remain hidden in routine examinations. I began this research with the expectation that reports would explore the three categories of themes highlighted in the conceptual framework of this study, (i.e. isomorphic pressures, university characteristics, and quality management). The data were analyzed by hand using qualitative content analysis procedures. Each report was analyzed against the three categories of themes.

The second empirical study at this level was focused on understanding how leaders (rectors and vice-rectors) in Greek universities perceived the environmental pressures and to what extent they adopted quality management in order to improve their universities' performances (chapter 8). The questionnaire I used was based on the Malcolm Baldrige Award Criteria for Performance Excellence (2005 version), to collect data on quality management and isomorphism. Questionnaires were sent to all rectors and vice rectors in all Greek public universities. The MB criteria enabled me on the one hand to study university leaders' perceptions of quality management, while on the other hand, to understand the extent to which it was being implemented at the meso level. Besides, this instrument facilitated an understanding of the leaders' perspectives with regard to criteria for performance improvement in relation to neoinstitutional pressures. This study was planned as a sequential quantitative and qualitative (QUAN $\rightarrow$ QUAL) study; however, I was unable to complete the second qualitative (QUAL) part of this study, as most participants were unwilling to participate in the second phase of the study. Therefore, the final design of this study was a concurrent quantitative (QUAN) one. The quantitative overall results were integrated in the final phase (integration).

To gain deeper understanding of the studied phenomenon at the micro level, I incorporated two more empirical studies. The first one (chapter 9) was a concurrent quantitative and qualitative (QUAN+QUAL) survey on department heads' perceptions and concerns about quality management. To study the isomorphic pressures that all 266 department heads in Greek universities experienced in deciding whether to adopt quality management practices, I employed an electronic survey largely modelled on the questionnaire by Ursin (2007, personal communication). In Finland, Ursin had conducted a survey to find out how quality assurance systems were understood by academic unit heads. My 
study was conducted in 2007, when the law concerning quality assurance in Greece came into force; thus, this study was able to provide a different angle of understanding of the phenomenon at both meso and micro levels.

The final empirical study at the micro level was a qualitative (QUAL) study (chapter 10). It was developed to gauge the extent of use of ISO models at the laboratories and academic support services in universities and simultaneously to examine isomorphic pressures that might influence the adoption of this particular quality management practice. This study included all available ISO cases in all Greek universities through interviews with directors of the laboratories and academic support services concerned. The inferences of these two studies in chapters 9 and 10 were also integrated into the final integration phase.

As noted in Teddlie and Tashakkori's (2009, p. 286) view, as well as others', the most important step in any mixed method study is "when the results from the study's QUAL and QUAN strands are incorporated into a coherent conceptual framework that provides an effective answer to the research question". Consequently, the final phase of this study was related to the integration of the data of the whole study. To answer the research question, I grouped the findings by detailing the corresponding qualitative and quantitative questions related to the explored factors influencing the adoption (if any) of quality management in Greek higher education.

During the analyses in each of the empirical studies, I followed Onwuegbuzie and Teddlie's (2003) suggestions. In addition, I analyzed quantitative data qualitatively and vice versa. I also developed an appropriate "construction technique" that helped to identify isomorphism and adoption of quality management at different levels.

In the integration, I took into consideration inferences from the EUA-IEP study (chapter 7) to triangulate the data provided by rectors (chapter 8) and department heads (chapter 9) from the same universities. Another view in integration was to examine if there were any evidence of universities using quality management, such as EUA evaluation (chapter 7) and ISO (chapter 10).

The final step was to verify if the empirical evidence coincided with the theoretical lenses and whether the neo-institutional perspective adopted for this study related to the answer of the fourth and final research question. Additionally, integration (chapter 11) was designed to include a section on recommendations for future avenues of quality management research in Greek higher education and to conclude with "the future challenges and changes in 
Greek higher education", whose aim is to provide the latest considerations on quality management in Greek universities.

\subsection{Sources of Data and Methodical Approaches}

The empirical part contains five empirical studies and an environmental scanning, using several methodical approaches for data collection. The pluralism in approaching the relationship between adoption of quality management and type of isomorphic pressure, as noted, stemmed from the nature of the problem addressed. The following sections cover the different empirical methods used in this study.

\subsubsection{Content Analysis}

Lincoln and Guba (1985) stated that documents are easily analyzed and are a stable source of rich information. Documents such as newspapers, minutes of meetings, and personal journals are valuable sources of information in qualitative and quantitative research (Creswell, 2005). According to Krippendorff (1980, p. 7) "content analysis is one of the most important research techniques in the social sciences, that seeks to understand data not as a collection of physical events but as symbolic phenomena and to approach their analysis unobtrusively". I chose content analysis to examine newspaper articles (chapter 6) and evaluators' reports (chapter 7). To analyze these sources I followed suggestions on content analysis that applied similarly to both studies.

Krippendorff (1980) distinguished five different ways of defining units for content analysis: physical, syntactical, referential, propositional and thematic. Krippendorff maintained that the purpose of the research is important in judging which kinds of units are most meaningful. My two studies took different approaches to content analysis. The first was a study of the mass media, the other an in-depth analysis of eight reports. The unit of analysis in the media study included the newspaper articles as a whole and it involved 751 articles. The unit of analysis in the second study was the evaluator's report. The EUA-IEP reports were obviously defined as physical units; while newspaper articles are syntactic units in Krippendorff's terms; others simply call them 'message units' besides syntactic ones (Rourke et al., 2001).

Analysis took place within those units. In both studies the analysis involved finding statements about facts (on quality management, on pressures on the universities, etc.) or opinions (of the journalists or of EUA-IEP evaluators). This 
lower level of analysis can be seen as taking place on thematic units (statements may be of different length and complexity, ranging from parts of a sentence to several paragraphs). Boyatzis (1998, p. 5) claimed that "thematic analysis enables scholars, observers, or practitioners to use a wide variety of types of information in a systematic manner that increases their accuracy or sensitivity in understanding and interpreting observations about people, events, situations, and organizations". Boyatzis (1998, p. 12) mentioned that there are three major obstacles or threats to using thematic analysis effectively in research: the researcher's projection, sampling, and mood style. My theoretical framework derived from chapters 2 and 3 and the operationalization of concepts from which it is made up, guards against those dangers.

The media analysis had a stronger emphasis on converting the qualitative data to numbers using a "quantitizing" technique described by Miles and Huberman (1994) than the study of the EUA-IEP reports, in which the emphasis was on a qualitative analysis.

\subsubsection{Survey}

The other main method to collect data was the survey approach. There are a number of advantages to survey research. Patton (1990, p. 14) stated that "the advantage of a quantitative approach [using surveys] is that it's possible to measure the reactions of a great many people to a limited set of questions, thus facilitating comparison and statistical aggregation of the data". However, besides closed questions designed for such quantitative analysis, I included open-ended questions in the surveys developed for chapters 9 and 10 for respondents to freely express their views and opinions on different subjects, thus adding a qualitative element as well. The final open-ended survey questions (chapters 8, 9, \& 10) allowed each respondent to make additional comments about any related subject.

Another reason for using the survey technique was that surveys in principle allow for generalization of findings and thus foster a great understanding of the larger population from which the sample was initially selected (Babbie, 1990, p. 42). Surveys can be used to identify attributes of a population from a small group of individuals (Fowler, 1993). Babbie (1990, p. 118) even stated that "the purpose of survey research is to generalize from a sample to a population so that inferences can be made about some characteristics, attitude, or behaviour of this population". Creswell (2003, p. 154) saw as "the advantages of surveys designs, ... the economy of the design and the rapid turnaround in data collection". 
Survey studies are limited by the "willingness" of the respondents to provide accurate responses. Moreover, the respondents may not have sufficient knowledge to answer all the survey questions adequately or may distort their responses due to personal biases or distrust of the study. These are considerations for possible bias and errors in measurement. Unfortunately, this is an inevitable characteristic of survey methods and therefore, I must rely on the respondents' honesty, accuracy, professionalism, and knowledge of self-rating. Survey information is sometimes seen as very superficial (Kerlinger \& Lee, 2000) and that might be a limitation of this study method, which of course was one of the reasons to integrate them in a mixed method design rather than as the single method of data collection.

Asking about a specialized topic, knowledge of which may not have been extensive throughout the universities, I had to find the most appropriate individuals to answer my questionnaire. Thus, I followed Kraut's (1996) suggestions for organizational surveys. The issue in this study was to understand how Greek universities deal with organizational pressures and how specific levels (meso and micro) within these universities may have multiple ways of dealing with institutional pressures to adopt quality management. The executives (rectors or vice-rectors, deans, respectively directors or administrators) of each level were perceived as the appropriate individuals to offer detailed information of the phenomenon under study. Kraut (1996, p. 154) regarding this fact wrote: "I believe a stronger involvement is fostered by encouraging executives to take part whenever possible".

In this study, I administrated three types of surveys: mail survey, web survey, and telephone interview. I made use of suggestions from Babbie (1990, 2001), Dillman (2000), Fink (1995), and Salant and Dillman (1994) on the medium of the survey.

A mail survey was chosen when many questions had to be asked of many respondents who were not easily reached by telephone or e-mail (rectors and vice-rectors, see chapter 8). In that case, the questionnaire consisted of 73 questions, and for clarity and succinctness, it was better to have institutional leaders read and answer their individual copy than to answer questions via telephone or by web survey. Mangione (1995) suggested using mail surveys when the research sample is widely distributed geographically. Other reasons suggested by Mangione (1995) and applied in my case were that, firstly, the research sample has made a moderate to high investment in the topic; secondly, I wanted to give my research subjects privacy in answering; thirdly, the questions work better in a visual format rather than in oral mode, and finally I had limited 
manpower (or support) to conduct the study. Mangione (1995) also remarked that the mail survey's advantage is that it allows the respondents to answer questions at times that are convenient to them. This advantage is related also to e-mail survey.

Concerning e-mail surveys, Dillman (2000, p. 354) stated that "an e-mail survey, at present, is little more than a simple text message and its construction may require computer skills no greater than those needed for composing and sending a message to a friend". The efficiency of electronic surveys includes "the nearly complete elimination of paper, postage mail out and data entry costs" (Dillman, 2000, p. 352). Additionally, Dillman (2000, p. 353) claimed that "in some instances these technologies may result in a decision to survey an entire population rather than only a sample". In other respects, the strengths and weaknesses of e-mail surveys are very much like those of conventional mail surveys. In my case (chapter 9), the e-mail survey was designed to collect data from the department heads of all 266 departments at twenty-one Greek universities. Czaja and Blair (2005, p. 228) pointed out a drawback in e-mail surveys: "because many computer viruses are spread by attachments, many respondents are wary of opening any attachment sent from some person or organization they do not know". Besides, although for computer-literate persons designing a questionnaire may not be difficult, as Dillman contended, persons in leading positions in universities may be of a generation for whom computer use even in answering a questionnaire still is an innovation.

A telephone interview was regarded to be the most appropriate technique for the data collection in chapter 10. I had used the other survey techniques in previous studies and in both cases where I employed mail and e-mail surveys, the response rate was low, which coincides with what other social science studies researchers also had found about Greece (see the beginning of section 4.2). Therefore, beyond the internationally acknowledged disadvantages of this technique, as described by Czaja and Blair (2005), I found that in such surveys, telephone communication or face-to-face interaction are most appropriate in Greek culture. Also, Fowler (1993, p. 105) emphasized the role of the interviewers, who indeed affect surveys: "They play a major role in the response rate that is achieved, they are responsible for training and motivating respondents, and they must handle their part of the interview interaction and question-and-answer process in a standardized, nonbiased way". Fowler (1993, p. 105) also suggested that interviewers have to get in touch with respondents in order to enlist cooperation, and he pointed out that the difficulty of this part differs greatly for each sample, and-we might add-each country. Telephone surveys share with face-to-face interviews the possibility to probe or ask for additional explanations if answers to the original 
questions are not sufficiently clear; in that way higher quality of responses may be achieved. This applies even if, as in my case, the survey in principle is a fully structured questionnaire, much like a mail survey.

To increase the response rate of the survey, Czaja and Blair (2005) and Dillman (2000) suggested a three-phase follow-up to increase response rates, which I followed in the mail and e-mail studies.

Numerical data were first of all analyzed quantitatively through descriptive statistics. Creswell (2003) suggested that organization of data is critical in qualitative research, and Miles and Huberman (1994) pointed out that there is no single accepted approach to analyzing qualitative data, although several guidelines exist for this process. I analyzed the data by following Creswell's (2003) and Miles and Huberman's (1994) suggestions, namely: developing a matrix or table of sources that can be used to help organize the material, preliminary exploration of data reading through the transcripts and writing memos, coding the data by segmenting and labelling text, using codes to develop themes by aggregating codes, connecting and interrelating themes, comparing themes and categories, describing and developing themes from the data, and representing findings. I developed matrices to organize the data. As the data were small ('small' meaning less than 500 pages, according to Creswell, 2003) in all cases, I used manual analysis.

\subsection{Ways and Criteria for Evaluating the Quality of the Entire Study}

As a methodology, mixed methods are widely accepted because a mixed methods research design provides a means to facilitate and explain several complex research phenomena across various disciplines. Creswell $(2009$, p. 106) noted that "the field of mixed methods will continue to expand across disciplines", and he anticipated seeing uniquely combined mixed methods designs in future papers. Additionally, Tashakkori and Creswell (2008, p. 294) argued for the use of mixed methods, along with many other scholars (Creswell \& Plano Clark, 2007; Greene, 2007; Johnson \& Ownuegbuzie, 2004; Teddlie \& Tashakkori, 2009). In varying degrees, all of them advocate the empowerment of the next generation of researchers to examine issues and research problems from multiple methodical perspectives. However, despite the increasing utilization of mixed methods in social science research, there is a dearth of systematic literature on the quality (and transferability) of inferences in such research (Teddlie \& Tashakkori, 2009). According to Tashakkori and Teddlie (2008, p. 101) the term "inference quality is 
introduced as an umbrella term for evaluating the quality of conclusions that are made on the basis of the findings in a study".

Onwuegbuzie and Tebllie (2003) pointed out that mixed methods research at that time remained a controversial approach to doing research in the social and behavioral sciences. Hence, researchers undertaking mixed methods techniques should seek to explicitly defend the approaches they are employing. Plano Clark et al. (2008) observed that there is a lack of literature providing guidance for researchers wanting to translate methodological discussions about mixed methods into practice. O'Cathain et al. (2008) stated that there is no consensus on criteria for appraising the methodological quality of mixed methods research. Validity and reliability of mixed methods research have, in the meantime, been discussed by several authors (Creswell, 2003; Creswell \& Plano Clark, 2007; Dellinger \& Leech, 2007; Onwuegbuzie \& Johnson, 2006; Tashakkori \& Teddlie, 1998, 2003). For instance, Onwuegbuzie and Johnson (2006) suggested the term legitimation to be used in mixed research to refer to research quality (in qualitative research the generally agreed upon term is "trustworthiness" and in quantitative research the term is "validity"). They suggested nine types of legitimation: sample integration, insider-outsider, weakness minimization, sequential, conversion, paradigmatic mixing, commensurability, multiple validities, and political. They argued that these types need to be studied more closely in order to determine when and how they operate and how they can be maximized. The authors also noted that mixed methods researchers should keep in mind that legitimation represents a process that is analytical, social, aesthetic, emic, etic, political, and ethical and which must involve the community of qualitative and quantitative scholars alike who are committed to addressing the multiple problems that can occur in mixed methods research. Onwuegbuzie and Johnson (2006) stated that this is the only way that the promise of mixed research can be realized in research practice.

Tashakkori and Teddlie wrote extensively about inference quality (Tashakkori \& Teddlie, 2003; Tashakkori \& Teddlie 2008; Teddlie \& Tashakkori, 2009). The authors argued that because of "ostensible obstacles" researchers must employ three sets of checks to assess the quality of their inferences:

- evaluating the inferences made on the basis of QUAN data using QUAN standards,

- evaluating the inferences made on the basis of QUAL data using QUAL standards,

- assessing the degree to which the meta-inferences made on the basis of these two sets of inferences are credible. 
Teddlie and Tashakkori (2009) underscored that the last point is especially difficult when the two sets of inferences are inconsistent. The authors suggested that two broad families of criteria for evaluating the quality of inferences may be generated: design quality and interpretive rigor.

Teddlie and Tashakkori (2009) noted that they found that the works of Onwuegbuzie and Johnson (2006) and Dellinger and Leech (2007) have elements in common with their own "integrative framework". Teddlie and Tashakkori (2009) noted however that "neither is able to provide a cohesive and comprehensive alternative for evaluating and improving the quality of inferences in MM research", and also stressed that "it is highly probable that a future set of standards for MM research will emerge, combining both models with [their] integrative framework (or others)" (p. 311).

Dellinger and Leech (2007) provided another perspective to the quality of a mixed methods approach, which they called the validation framework (VF). Their framework has five components: foundational element, elements of construct validation for quantitative, qualitative, and mixed research, inferential consistency, utilization element, and consequential element. Their proposed framework gives some practical suggestions and could provide a guide for organizing the necessary evidence needed to support data meanings in mixed methods studies.

Against this backdrop of the current literature on inference quality in mixed methods research, the following text provides the step-by-step process and criteria for assessing the multi-level mixed design that I used to explain isomorphism and adoption of quality management in Greek universities. As yet, there are very few published works available that use a mixed methods approach similar to my study; what follows are outlines of the sequence in which quality criteria were considered and applied in four phases: during data collection, during data analysis, during the inference per empirical study, and during the integration phase of the entire study.

\subsubsection{During Data Collection}

\section{Quantitative (QUAN) Data}

Quality issues in QUAN data collection relate to reliability and validity. To increase reliability I used structured interviews (Creswell, 1994; Merton et al., 1990). Besides, I pilot-tested the instruments in empirical surveys (chapters 8 \& 9). In chapter 9 , the survey tool had already been used, which added reliability to the study. 


\section{Qualitative (QUAL) Data}

Merriam (1998) pointed out that qualitative research methods theorists suggest that the multiple data collection and data analysis methods employed create research that is more valid. Teddlie and Tashakkori (2009, p. 303) when discussing quality during the QUAL data collection in mixed methods studies, focused on credibility and dependability. Credibility has been used as a QUAL analogue to internal validity (Lincoln \& Guba, 1985, p. 300). Teddlie and Tashakkori (2009, p. 332) noted that dependability "is a QUAL analogue for the QUAN concept of reliability and it [is] concerned with the extent to which variation in a phenomenon of interest can be explained consistently using the 'human instrument' across different context[s]". Lincoln and Guba (1985, p. 317) emphasized "inquiry audit" as one measure which might enhance the dependability of qualitative research. Furthermore, Lincoln and Guba (1985, p. 170) enhanced Teddlie and Tashakkori's argument for dependability by stating that "dependability" or "consistency" of the results could be substituted for "reliability" and suggested that dependability can be achieved by stating the investigators' position, by using multiple methods of data collection and analysis (triangulation), and by describing in detail how data were collected. The QUAL data collection activities in my study related to document analysis (chapter 6 \& 7), and interviews (chapter 10). The conceptual framework and the research question discussed in chapter 3 helped me in this pluralistic project in order to study the same phenomenon. Issues of credibility and dependability are covered respectively in chapters 6,7 , and 10 .

\subsubsection{During Data Analysis}

In QUAN studies, quality criteria for overall inferences were related to appropriately adequate analytic strategies. In chapters 8 and 9, descriptive statistical analysis of the main survey results was performed.

In QUAL studies, quality issues are related to appropriate adequate and analytic strategies. According to Creswell (2003), three primary forms typically used by qualitative researchers are triangulation, member checking, and auditing. Creswell and Plano Clark (2007) stated that in qualitative research validity, comes from the analysis of the researcher and from information gleaned from participants and external reviewers. Member checking is another process for assessing qualitative validity in which the researcher asks one or more participants in the study to check the accuracy of the account (Creswell, 2003). According to Creswell (2003), the member checking process was employed in 
instances of an ISO empirical study. In my ISO study, at the end of the interview, I summarized the interviewee's rationale for adoption of ISO standards in order to verify the accuracy of my understanding. The reliability of the findings through triangulation of the interview was another strategy used this approach in ISO cases. In the empirical study in chapter 6, inter-coder reliability was checked; but not in the empirical study in chapter 7 , as the type of pressure was only of one type.

\subsubsection{During the Inference}

During the inference in each empirical case, I applied guidelines that were derived from mixed methods literature. In quantitative studies, these conclusions were based on quantitative data analysis results, such as internal validity, statistical conclusion validity, some aspects of construct validity, and external validity.

In qualitative studies, these conclusions were based on qualitative (QUAL) data analysis results, such as some aspects of credibility/confirmability and transferability. Creswell and Plano Clark (2007, p. 146) suggested that in mixed methods the inference validity is also enhanced when the researcher shows which potential threats to validity that arise during data collection and analysis were already alleviated. I took into consideration issues such as: I selected all rectors and vice-rectors from Greek universities as well as all department heads to participate in this research. The selection of such meso and micro level leaders while conclusions were drawn about the universities and units as a whole may prove to be a limitation of this study, though rectors and department heads are professors/members of faculty and I viewed their perceptions as linked with the faculty members. The combination of theory used, methodological triangulation, data analysis triangulation and transformation in the design of the study was designed to enhance the credibility and validity of the research (Creswell \& Plano Clark, 2007; Tashakkori \& Teddlie, 1998; Teddlie \& Tashakkori, 2009).

Teddlie and Tashakkori (2009, p. 292) stated that "quality issues in mixed research must be discussed in the context of the correspondence between the meta-inference and the stated purposes for using a MM design". Additionally, the authors noted that "a strong inference is only possible if there is an appropriate design that is implemented with quality" (p. 300). The way the multi-level mix was designed, as presented above, reflects its design quality and meets the research criteria defined by Teddlie and Tashakkori (2009, p. 301), i.e., design suitability, design fidelity, within-design consistency, and analytic adequacy. 


\subsubsection{During Integration}

Finally, in the overall integration I provided answers to the following questions related to interpretive rigor, forwarded by Tashakkori and Teddlie (2003) and Teddlie and Tashakkori (2009) and summarized in Table 4-2. Greene (2007, p. 174) noted that the "knotty issue of judging the quality of inferences yielded by a study with multiple and interactive assumptions and stances remains a conceptual and procedural challenge".

Table 4-2 Quality criteria for interpretive rigor

\begin{tabular}{|c|c|}
\hline $\begin{array}{l}\text { Interpretive } \\
\text { Consistency }\end{array}$ & $\begin{array}{l}\text { 1a) Do the inferences closely follow the relevant findings in } \\
\text { terms of type, scope, and intensity? } \\
\text { 1b) Are multiple inferences made on the basis of the same } \\
\text { findings consistent with each other? }\end{array}$ \\
\hline $\begin{array}{l}\text { Theoretical } \\
\text { Consistency }\end{array}$ & $\begin{array}{l}\text { 2) Are the inferences consistent with the theory and state of } \\
\text { knowledge in the field? }\end{array}$ \\
\hline $\begin{array}{l}\text { Interpretive } \\
\text { Agreement }\end{array}$ & $\begin{array}{l}\text { 3a) Are other scholars likely to reach the same conclusions on } \\
\text { the basis of the same results? } \\
\text { 3b) Do the inferences match participants' constructions? }\end{array}$ \\
\hline $\begin{array}{l}\text { Interpretive } \\
\text { Distinctiveness }\end{array}$ & $\begin{array}{l}\text { 4) Is each inference distinctively more credible/plausible than } \\
\text { other possible conclusions that might be made on the basis of } \\
\text { the same results? }\end{array}$ \\
\hline $\begin{array}{l}\text { Integrative efficacy } \\
\text { (mixed and multiple } \\
\text { methods) }\end{array}$ & $\begin{array}{l}\text { 5a) Do the meta-inferences adequately incorporate the } \\
\text { inferences that are made in each strand of the study? } \\
\text { 5b) If there are credible inconsistencies between the } \\
\text { inferences made within/across strands, are the theoretical } \\
\text { explanations for these inconsistencies explored, and possible } \\
\text { explanations offered? }\end{array}$ \\
\hline Interpretive & 6a) Do the inferences correspond to the stated \\
\hline Correspondence & $\begin{array}{l}\text { purposes/questions of the study? Do the inferences made in } \\
\text { each strand address the purposes of the study in that strand? } \\
\text { 6b) Do the meta-inferences meet the stated need for utilizing a } \\
\text { mixed methods design? (i.e., is the stated purpose for using a } \\
\text { mixed-methods design met?) }\end{array}$ \\
\hline
\end{tabular}

Source: Teddlie and Tashakkori (2009, p. 301-302) 


\section{Environmental Scan of Greek Higher Education}

This scan of the environment of Greek higher education follows the previously indicated division into legal/political, economic, European, technological, and sociocultural indicators. In this study, the purpose of scanning the Greek higher education environment is to integrate knowledge from disparate sources about various environmental elements in order to build knowledge about environmental factors which may influence the adoption of quality management practices. In this context, it is crucial to highlight both external and internal elements of the environment that might influence organizational change.

The first section begins with a brief introduction and a historical setting of Greek higher education; then, the section continues with issues regarding the external environment of Greek universities, followed by a look at the basic characteristics of these academic organizations. Thus, the internal environment is described in the second section. This chapter seeks to introduce the Greek higher education system and the external environment in which the Greek universities operated until 2006.

\subsection{General Information}

Greece is a country of less than 11 million inhabitants occupying an area of 131,990 square kilometers. It is situated on the Balkan Peninsula and includes numerous islands in the eastern part of the Mediterranean Sea, in the Aegean Sea, and in the Ionian Sea. During the academic year 2003-2004, according to the Greek Ministry of Education's statistics, the student population was 352,936. Detailed information concerning this population can be found in the section sociocultural elements.

The first Greek university was founded in Athens in 1837 with four faculties, the Hellenic University of Otto. In 1873, the National Technical University was established, also in Athens. After the turn of the twentieth century, other universities were established.

The institutional structure in Greece (Hellas) is as follows:

The Hellenic higher education system comprises two sectors (Law 2916/2001): The university sector, which consists of 23 universities (including the Open University) and the technological sector, which consists of 16 Technological Education 
Institutions (including the School of Pedagogical and Technological Education). [...] Moreover during the period 2004-2006, 13 new Departments have been established in various universities and 4 new Departments have been established in 4 TEIs, whereas three more Departments will operate in 2007-2008. Higher education is also provided by Higher Ecclesiastical Schools (Ministry of National Education and Religious Affairs), Merchant Marine Academies (Ministry of Mercantile Marine), the Higher Military Education Schools (Ministry of Defense), and the Higher Police Academies (Ministry of Public Order). However, all the above institutions cannot offer postgraduate study programmes but only in cases of cooperation with the universities which award the post-graduate degrees. (YPEPTH, 2005, p. 4)

When speaking of higher education in Greece, most people refer solely to the university sector (AEI) and likewise, this book only deals with the 21 universities. On the Ministry of Education's webpage, remarkably, there is a "zoning distribution" of universities8, which categorizes universities as 'urban' or 'peripheral' in regards to their location. Also, in some official documents, universities are characterized as either monothematic or multidisciplinary. All universities are research universities and they offer Masters and Ph.D. programs. The following characterizations are derived from these (semi-)official distinctions among the universities.

\section{Age}

The first Hellenic University was founded in Athens in 1837, as was just mentioned. After the turn of the twentieth century, other universities were established. In 1982, an effort was made to bring university education up-to-date in Hellas with the passage in Parliament of a new law, which laid down a completely new framework of operation for the universities and paved the way for establishment of new universities. Thus, in terms of age, there are two groups of universities, old (1837-1982) and new (1982-2006). There are fifteen old universities and six new ones.

\section{Size}

The number of new students allowed to enrol in each university is determined by the Ministry of Education. Three size categories of universities for freshman students are: small, below 1,000; medium, 1,001-3,000; and large, over 3,000.

\section{Location}

Geographical diffusion is another factor. In Greece, the only cities with over 1,000,000 habitants are Athens and Thessaloniki. Following the ministry's

\footnotetext{
${ }^{8}$ http://www.ypepth.gr/el_ec_page222.htm, accessed 14 August 2008
} 
distinctions, universities are "urban" if located in Athens (including Piraeus city) and Thessaloniki. All others are known as peripheral.

\section{Types and Range of Studies}

The disciplinary fields and their study programs that are offered by universities encompass a broad range spectrum. The eight disciplinary fields used mostly in official statistics are: 1 . Sciences; 2 . Engineering; 3. Medicine and Life Sciences; 4. Law; 5. Behavioural Sciences; 6. Social Sciences and Economics; 7. Humanities; and 8 . Arts. The range of studies offered at a university can be defined as either multidisciplinary (a wide range of subjects) or monothematic (a specialized institution). These terms will be used in the next chapters because there are seven monothematic universities in Greece. This group is comprised of two technical universities, two economic universities, one agriculture university, and one school of fine art. Meanwhile, the other 14 multidisciplinary universities cover from 2 to 8 of the disciplinary fields. This study excludes the Open University, which offers distance learning with tuition) and the International University (which only began to operate in 2007, after the period studied here).

\subsubsection{Legal and Political Issues in Greek Higher Education}

The higher education system in Greece, and especially its university sector, is ruled in principle by Article 16 of the Greek Constitution, valid since 1975, as well as Parliament's frame-law 1268/1982. Although universities were organized and operated for a long time under the Article 16, an effort was made in 1982 to bring university education up-to-date in Hellas, through a completely new framework of operation for Greek universities. Karmas et al. (1988, p. 264) stated "the year 1982 will remain a historical landmark for university education because Greece abandoned a model of university government based on Central European experiences and practices of the past, which had remained in operation for over 50 years". This law accounts for the major and most significant reform in Greek higher education since 1932. A significant number of laws have followed since 1982, with partial improvements and supplements to the frame-law.

The major principle of Article 16 of the Greek Constitution (see Appendix A) is that the privileges of full autonomy and of academic freedom are reserved for the universities. At the same time, the Greek Constitution refers also to the state control upon the universities, which is carried out by the Ministry of National

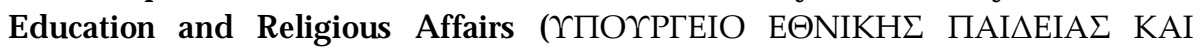


$\Theta P H \Sigma K E \Upsilon M A T \Omega N-Y \Pi E \Pi \Theta^{9}$ ). The need for state control stems primarily from the fact that, according to the Greek Constitution, universities are state-financed institutions. As in most countries, the equilibrium between university autonomy and state control is quite a difficult affair and causes many problems and friction in the relations between the universities and the government. Saitis (1988, p. 261) declared: "in theory there is 'absolute' independence for university institutions to manage their affairs as they wish; in practice, however, the sense of 'selfadministration' has disappeared because all university decisions need ministerial approval".

According to the Greek Constitution, universities are autonomous institutions, yet their mission is determined by the law, and therefore is uniform across all universities. According to one EUA-IEP evaluator's report (to be treated in more detail in chapter 7), a Greek university's mission and scope was:

to provide the necessary means for a successful professional career both for its students and for its academic and administrative personnel; to contribute to satisfying the social, cultural and development needs of the country.

Other EUA-IEP evaluators, looking at another university, found that:

The broadness of the mandated mission hardly distinguishes "University A" from any other university. This mission statement is typical for Greek universities: they do not see room to deviate in their mission statement (not even by making it more specific, or giving it a local twist) from what is given by the laws.

Furthermore, the Greek Constitution stipulates that university studies be free of charge. According to this principle, there are no fees for Greek undergraduate students. Nevertheless, the most recent legislation (2083/1992) provides the possibility of setting tuition fees for postgraduate studies. It must also be noted that the stipulation is usually interpreted broadly and extends to many other issues, which include provision of free textbooks and free room (for low income students) and board. Article 16 stipulates that university education is public and can only be offered by the state. As a result, there is no possibility for private universities to be established in Greece. However, the issue remains contentious in debates on future constitutional reform. Despite explicit constitutional restrictions, a large number of private schools in Greece claim that they offer courses at the university level; however, their degrees are not recognized as such by the Greek State. These private schools (officially called "Centres of Liberal

9 From October 2009 name its changed to: Ministry of Education, Lifelong Learning and Religious Affairs. 
Arts") do not meet the minimum legal or essential qualitative requirements for providing university-level courses. Nevertheless, they have developed contractual cooperation with universities in other countries to establish indirect pathways for the recognition of their courses. The Greek State's insistence on refusing to legalize these indirect pathways has already generated tension between the Greek State and the European Court of Justice. This complicated problem arose mainly because of the promptness of foreign universities to develop contractual cooperation with all these private schools while at the same time, ignoring both Greek legislation and minimum guarantees for quality.

The period 2006-2007 proved to be the year when the Greek higher education system was hotly debated even though the Greek National Educational Council had already proposed changes in educational policy for higher education (Article 16 of the Constitution). These issues are covered very briefly in chapter 11 .

The frame-law 1268/82 entitled "The structure and Operation of Higher Education Institutions" introduced a new model for the operation of the Greek universities and remained valid until March 2007. Karmas et al. (1988, p. 264) stated that the frame-law 1268/82 "makes [each university] department the basic functional academic unit, with a number of related departments grouped into schools; for the first time in Greece, it allows the creation of Graduate Schools for the development of meaningful postgraduate programmes of study and research; it divides the academic year into two separate semesters; it requires completion of a certain number of credits for graduation".

Photo 5-1 Highlighted articles in the $6^{\text {th }}$ Edition Frame-Law 1268/82

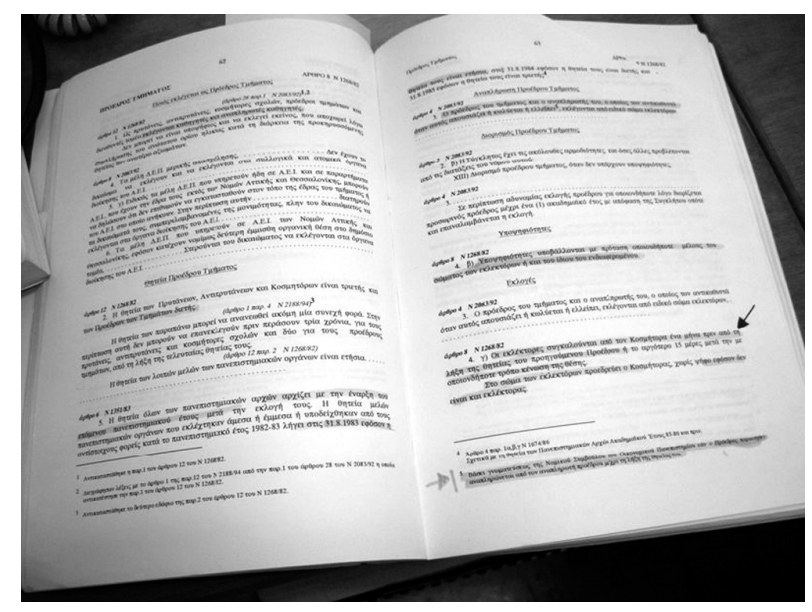


Every department and every unit in a university operates in accordance with this frame-law. Its text is found on every administrator's desk, and highlighted chapters and paragraphs (picture 5-1) show the real use of this law. In every board meeting or assembly, administrators keep notes and always carry the new, $6^{\text {th }}$ edition of the text.

The $6^{\text {th }}$ Edition (Benos, 2003) consists of 445 pages, 9,643 paragraphs, 120,401 words, or $3,437,216$ characters. Until changes in 2006 , this edition held the requirements, laws, rules, and regulations that guided every single process in Greek universities.

The content of this edition includes sections such as:

- General rules: Organizations of the Higher Education, Academic Freedom, Academic Asylum

- Self-government of Higher Education

- Organization of the University

- Decision-making

- University Body

- University Personnel

- Undergraduate Studies

- Graduate Studies

- Students Issues

- University Research

- Greek Open University

- International University for Greek Studies

- Research Committee

- Transitive Provisions (stipulation)

- Final Provisions

A significant number of laws have been added since 1982, although these subsequent modifications did not affect the overall philosophy, values, and basic principles set up by frame-law 1268. On the one hand, the frame-law considered values and principles of democracy, collective participation, accountability, and transparency; on the other hand, it considered the rationale of the organizational, personnel, and educational structure of the Greek higher education system. Moreover, 1268/1982 provided one of the strongest participatory roles for students when it is compared to other countries, because it allows students to have an active role in decision-making, governance, strategic planning, and quality assessment. In addition, the frame-law established the academic asylum in universities' areas and that resulted from the violent incidents during the 1973 student uprising that took place at the School of Law and at the "Metsovio" 
Technical University in Athens. The academic asylum "was later encroached, which resulted in serious damages to the institutions as well as in a number of other unlawful actions" (Kyriazis \& Asderaki, 2008, p. 42).

Some brief remarks about the history of higher education on the political agenda, partisan politics, and politicization of the university are also necessary. By the late seventies, two parties with competing charismatic leaders, namely the conservatives ("Nea Dimokratia") and the socialists ("PASOK"), managed to establish a long-lasting domination of the political scenery. They further succeeded in building up strong, wide-reaching and centralist party organizations that rapidly "colonized" Greek society and institutions. This unprecedented wave of "partification" seemed to challenge the kind of traditional "backstage localism" that had characterized Greek politics from the very beginning of Greek statehood. In Greece from 1974 until now (2010), three major reforms of higher education took place, under two different political parties. However, "it is very common for both political parties to oppose reforms only because the other party proposed them" (Nakos \& Hajidimitriou, 2009, p. 71). The major higher education reforms can be grouped in terms of the policy concepts of "democratization-1982", "quality-2005", "efficiency-2007", and "performance200710". During this period (1982-2007), additional legislative work took place which was reflected in a management model directed ideologically and politically by the two different main political parties. These reforms led to adaptation of Greek higher education policy, however, these reforms were criticized initially for their inefficiency and ineffectiveness, which reveals how much Greece was characterized by its political dynamics. Saitis (1988, p. 249) regarding the framelaw 1268/1982 stated that "bills were drafted on the basis of hastily prepared reports and there was no evidence that systematic research was conducted into problems and needs of university education. Nothing comparable occurred, for instance, to what happened in Great Britain at the time of Callaghan's Great Debate. He also noted that "because of haste generated by political opportunism, the implementation of Education Acts is very often confused. Laws are frequently vague and complex and fundamental problems are left unresolved". Saitis (1988, p. 252) also mentioned a practical side of educational policy in Greece. He observed that "from the foundation of the Ministry of Education until the present [1988], 288 Ministers have been appointed, which means that on average they have each served about seven months" (similarly Papandreou, 1998, p. 24). Saitis mentioned that the short tenure of the Ministers "is not only the main problem of short-term policy-making". The author noted that beside the day-to day routine activity, "the Minister also has to spend an enormous amount of time both in Cabinet and at his political party's meeting, which frequently have little to do

10 These issues are covered very briefly in chapter 11 
with education" (p. 253). He also noted that "the decision-making process itself lacks adequate national consultation or responsiveness to the views of a wide cross-section of the population, and it suggests that the process is more arbitrary than democratic" (p. 254).

Karmas et al. (1988, p. 264) noted that the frame law 1268/1982 "gives the right to the students and to other interested bodies of the university community to participate, for the first time in Greece, in matters concerning university government and in decision-making processes". The authors also noted that "sometimes ideological and political considerations have become the predominant criteria for making decisions: the most obvious (and perhaps most important) example is the election for a three-year term of the Rector and the two Vice-Rectors... Since 1982, when the new model of university government was introduced, the election of the Rector has become a political issue involving manoeuvres, alliance etc."(p. 265).

Saitis (1988, p. 257) also noted, "given that the laws and the presidential decrees change very often, the phenomenon of polynomy [plethora of laws] creates confusion in the management of both the MNEC [Ministry of Education] and the universities". The author further stated that "it is frequently difficult to know if a specific article of a law is or is not in force at a given time". The author also stated that "it is sufficient to say that day-to-day managerial control is the most fundamental form of the MNEC supervision over the universities; this supervision narrows the boundaries of university independence, serves political interests and introduces strong bureaucracy into relationships between Ministry and Universities" (p. 257). For Saitis' therefore "the structure of Greek higher education is strongly influenced by the political and administrative system in which it is embedded and especially by an entrenched concept of centralised authority" (p. 257). The author concluded that in Greece, the universities "do not enjoy a considerable degree of autonomy. There is no clear-cut definition of areas of responsibility and authority between State and universities and the latter do not have the power to manage their own affairs. At all times they are under political supervision because their affairs demand ministerial approval and their budgets are approved and implemented under the powers of the Ministry of Education and the Ministry of Finance" (p. 258).

Another element of the politics in Greek higher education was highlighted by Pesmatzoglou's (1994) view regarding government, ideology, and the university curriculum in Greece. His view "relied on existing written testimonies and oral communications since there was a grave lack of systematic quantitative and qualitative studies in this area. Even legislation affecting education has never 
been the result of all-encompassing studies of the state of university affairs; instead, it has tended to reflect the balance of power within governing party and within Academia" (p. 291). Pesmatzoglou (1994) first of all mentioned the political driver of "the continuous growth in undergraduate numbers", namely that "the level of admissions was not determined by the departments or the universities on the basis of educational criteria, but by the political expediencies of the party in power" (p. 292). Secondly, the establishment in various cities and islands of "post-secondary institutions baptized as "universities'" was "a second element linked to political expediency for the appeasements of local pressure". Pesmatzoglou (1994, p. 292) noted that these institutions "were purely partypolitical initiatives transcending the major government parties and certainly not the result of studies of epistemological developments or of economics, social and cultural needs of specific cities or regions".

Papandreou (1998, p. 23) also noted that "our [Greek] political heritage, moreover, is characterised by tradition of 'clientelism' ... a clientelistic relationship between the politician and the citizen. This has had its impact on education by developing clientelistic mechanisms that direct the educational processes, or by creating a very rigid mechanism to avoid clientelistic relationships". He also noted that "Greece is obviously a long way from making those qualities [flexibility, diversity, and decentralization-AP] part of its social and educational structures, and we have long way to go make the necessary changes in order to confront the challenges with which such transformation can come about" (p. 21).

As a result, Keridis and Sfatos (1998, p. 174) called the Greek university "a behemoth, unable to respond to the changing social environment and demands". According to them, politics also pervade internal decision-making in the universities, where:

strong interest groups ... are afraid of competition, responsibility, accountability, and meritocracy. They often find ideological and political support among those who are sincerely interested in safeguarding the equalizing qualities of the Greek State system, and often abuse democratic procedures and legacies in order to legitimize, and in a sense canonize, particularistic claims (Tsoukalas and Panagiotopoulou, 1992). These groups have a strong interest in over-politicizing and often polarizing a debate, even when small, technical measures are involved. Thus, the debate on educational issues in Greece becomes a metaphysical battle of good versus evil, Right versus Left, progressive versus reactionary. 
In this arena, the student body of the Greek universities is strongly politicized as well, and frequently acts in a partisan spirit. The student movement emerged in the mid-1970s from the seven-year period of dictatorship (for details see Antonopoulou 1991). Students are included in the University Board. They take part in the Government of the University in the senate, departments, faculties and section meetings. Pesmatzoglou (1994, p. 292) stated: "the third and perhaps the most pervasive influence arising from exogenous party-governmental populism was provided by the provisions on participation incorporated in the so-called Framework Act $1268 / 82 "$. Students have a very strong organization. Sometimes students organize protests not only concerning academic affairs, but also on other political issues. Often, students organize strikes from their classes, and at other times they occupy administration buildings, classes or barricade streets to give voice (and force) to their demands. However, rather than pressing for change towards the more entrepreneurial university (Clark, 1998) underlying Massalas' remarks as well as much of current policy initiatives, students seem to want to maintain universities and their own status as dissociated from society as they can. Dinas \& Gemenis (2007) noted that the most important Greek student unions are simply branches of the mainstream political parties. The authors also noted that "There are departments which could be called independent in the sense that either there is enough balance in the distribution of students' votes or there is no particular continuity in the outcome of the student union elections". However, "there are also many departments and even universities which could be viewed as safe constituencies for some unions, since there is a dominant student party which preserved its dominance during the years" (p. 14). Dinas and Gemenis (2007) also examined the reasons that determined participation in protest among Greek students in May 2006. Their hierarchical linear modelling showed that perceived cost/benefit calculations, selective incentives, pessimism, engagement, and post-materialism seemed to play an important role in explaining participation in protest activities. They measured the mobilization by the electoral appeal of partisan students' unions, but that did not seem to exert an important influence on students' decisions to engage in protest activities.

\subsubsection{Economic Elements}

All public universities in Greece are entitled to financial support (Law 2083/92, 2158/93, and 2327/95). The main sources of funding are the state budget and European funds. The Ministry of Economy and Finance, the Ministry of National Education and Religious Affairs, agree with the Conference of the Rectors of universities and the Presidents of TEIs on the amount of funds, the types of expenses (infrastructure, equipment, etc.) and the standards and guidelines for the four-year planning of higher education institutions (YPEPTH, 2007, pp. 3-4). 
Table 5-1 illustrates higher education finance figures for the years 2004 to 2007 in the regular national budget.

Table 5-1 Universities' regular national budget 2004-2007 (million euro)

\begin{tabular}{ccccc}
\hline Type of Budget & $\mathbf{2 0 0 4}$ & $\mathbf{2 0 0 5}$ & $\mathbf{2 0 0 6}$ & $\mathbf{2 0 0 7}$ \\
\hline Regular Budget & 826,80 & 869,80 & 928,50 & $1,036,35$ \\
\hline
\end{tabular}

Source: Ministry of Economy and Finance 2007 (cited in Kyriazis \& Asderaki 2008, p. 130)

To expand upon table 5-1, state financing is provided to the universities in three parallel ways. The first covers the salaries of the permanent personnel. The amount covering salaries is inflexible in the sense that the salary of every employee of the universities is determined by rank, years of service, and marital status in accordance with Law 2530/97. Hence, the universities have no autonomy in this regard. The basic monthly salaries of all ranks for teaching and research faculty members are derived from the basic salary of a lecturer through a set of multiplication factors (Table 5-2):

Table 5-2 Factors of the academics basic salaries

\begin{tabular}{l} 
Professors: 1,50 \\
Associated professor:1,30 \\
Assistant Professor: 1,10 \\
Lecturer: 1,00 \\
Professor assistant of the old system 0,90 \\
Assistant: 0,90 \\
Scientific Assistant:0,90 \\
Special Teaching personnel: 0,90 \\
\hline
\end{tabular}

Source: www.euridike.org

The second parallel way provides, on a lump-sum basis, funds to meet universities' functional/operational expenses (Regular Budget, Ministry of Education), and the third provides, on an earmarked basis, funds for universities' capital needs. This sum is to support investments (Public Investment Budget, Ministry of Finance). Research is funded separately (YPEPTH, 2005, p. 7).

Then, each individual higher education institution negotiates with the Ministry of Education about its annual budget, which is roughly based on an algorithm that takes into consideration the number of students and the academic staff, the number and the location of the departments, etc. (YPEPTH, 2007, pp. 3-4). Psacharopoulos (2002) commented that Greece utilizes a direct allocation model, 
meaning that public money goes directly to the university without any intermediate evaluation of their efficiency and in the absence of any concept of varying financial rewards depending on the quality of the offered education and/or the students' socio-economic background.

Although the funding formula is derived primarily from quantitative input factors, there is-as stipulated-room for negotiation. Kyriazis and Asderaki (2008, p. 129) euphemistically called this: "HEIs have the potential to be additionally funded by the budget for special or unscheduled funding". This whole process led some EUA evaluators to comment:

The system for resource allocation is not transparent in Greece. The rules for budget approval are cumbersome and slow, resulting in very late approval of the university's budget. Sometimes approval is postponed until the end of June for the current budget and as late as September for the investment budget. The university has very little room for maneuver in financial affairs. The funding method is still incremental budgeting, the relation between the Rector and the Ministry is a crucial factor. (EUA-IEP evaluators report for University B)

Universities then have to reallocate the government funds by setting up their own budget. The internal budget of each university then has to be approved by the Ministry of Education and by the Ministry of Economics. The state-control restrictions extend to financial management procedures, as all expenses have to be controlled by the regional or local economic authorities and every provision of large-scale equipment has to follow stringent time-consuming processes. These procedures were followed until 2006.

Figure 5-1 shows the expenditure on tertiary education in OECD countries as a percentage of GDP in 1999. The data cover all expenditures-direct and indirect, public and private-on universities and distribution of current and capital expenditure on educational public and private institutions involved in delivering or supporting tertiary educational services (EC, 2003, p. 217). Greece spent only 1.0\% of GDP spent on tertiary education, while Finland spent $1.8 \%$ and the EU average was $1.3 \%$. EU countries, though, spent markedly less than the USA.

Comparing the Greek economy with similar economies (in terms of public expenses as percentage of GNP) within the European Union (Eurostat, 2005) ${ }^{11}$, Greece dedicates a considerably smaller percentage to education than Portugal, Belgium, France, Austria, and the Netherlands. There is a discussion in Greece

\footnotetext{
11http://epp.eurostat.ec.europa.eu/portal/page?_pageid=1090,30070682,1090_31583003\&_dad=portal\&_s chema $=$ PORTAL, accessed April 2008.
} 
that the low level of public expenses for education is owed partly to the inelasticity of concrete categories of public expenses, such as defence and public safety, salaries of public employees, and subsidies of actuarial funds.

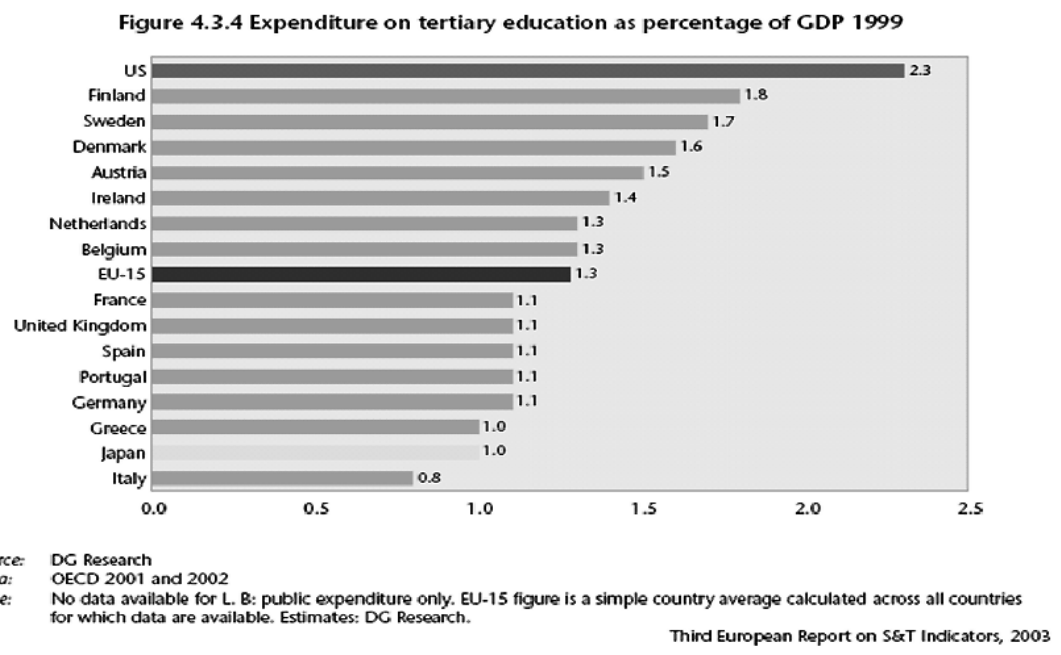

Figure 5-1 Expenditure on tertiary education as a percentage of GDP in 1999

Louloudis (2010) calculated that the public budget covered 97.9\% of Greek higher education and the private percentage was about 2.1\%, while in OECD countries these percentages were on average $75.7 \%$ and $24.3 \%$, and in $19 \mathrm{EU}$ countries $84 \%$ and 16\% (OECD, 2007, p. 221 in Louloudis, 2010, p. 155). He mentioned that "this percentage is well-known in Greece; however, we [Greek academia] do not attach sufficient importance to the percentage" (p. 155). He noted that universities in Greece know that there are private funding sources; however, in practice, some Greek universities are "rich" and can access private funds, others are "poor" and they remain "poor" because they cannot access or find private sources. Private sources can be attracted through research projects and sometimes the sums involved are greater than the university's regular public budget. Louloudis (2010, p. 157) noted that "this inequality between institutions must be recognized if we want to discuss seriously about underfunding".

\subsubsection{Financial Management Mechanism}

Greek legislation allows universities to develop entrepreneurial activities in both research and services. The Greek legislation encourages universities in such activities, as it provides them with a legal framework for financial management that 
is much more flexible than the one for state resources. This flexible financial management can be carried out through three different mechanisms.

- The first is the independent system of financial management for research funding from external sources. This system operates through the socalled Research Committee of each university, with responsibilities often extending to activities beyond research.

- The second mechanism is the Company for the Management of the Property of the University. This is a business-type institution, which belongs to the university and operates under the control of the Senate. This company operates on free-market terms and aims to maximize and utilize the institution's property and resources.

- The third mechanism refers to the possibility of the universities to establish Research Institutes associated with one or more of their faculties. Research institutes enable universities to improve the organization of their research activities principally in multidisciplinary or interdisciplinary scientific areas.

\subsubsection{Research Funding}

Individual and group research projects can be classified into two categories:

1. Projects without external funding: These are typically basic research projects whose purpose is publication of scientific articles and the development and recognition of researchers.

2. Funded research projects: These are mostly applied-research projects involving groups of researchers with a senior scientist (Principal Investigator) responsible for research output and its conformance to the research contract. The financial administration of those projects is the responsibility of the Research Committee, which provides a certain degree of flexibility.

Not only universities as a whole, but also laboratories have full autonomy in the way they organize and conduct research. The laboratories belong to two different categories according to their legislative status: those which have been established by a Presidential decree and those which have been informally established in order to satisfy the need for organized research. Formal laboratories have research resources and funds that come from their host departments. Therefore, they enjoy limited autonomy unless they manage to attract external funding. Faculty members also hold key positions in laboratories and direct or conduct research or participate in the development of entrepreneurial activities in Research Institutions or Technological Parks. 
A large number of research activities, mainly in the area of applied and technological research, are supported financially by outside resources in a contract-based partnership. In this case, funding follows the principles of orientation and competition.

The responsibility for the development of research policy belongs to the General Assemblies of the Departments and to the Senate of the university as a whole. A percentage of the total budget of each research project, not exceeding $10 \%$, is topsliced by the Research Committee of the university, which thereby becomes a major player in the planning of research policy and which is also responsible for the proposals to the Senate for the allocation of internal research funding. The approval of the financial management of a research project with outside funding produces additional resources for the university itself, which can be used in turn for financing specific educational development and research activities and moreover, in some cases, to produce research resources for less attractive or non-marketoriented departments and specializations. The initiative for all types of research projects lies with the academic personnel of the university. To this extent, sources of external funding vary by the attractiveness of the department's field of knowledge, along with the faculty members' capabilities to attract resources and support, and the quality of the services provided by the Research Committee of the university. As a consequence, the balance between teaching, research, and service activities varies among departments.

The specific processes that universities used to monitor the quality of teaching and research by 2006 were applied by the universities' own arrangements and regulations.

According to the Ministry of Development (2003)12 the bulk of research activities in Greece is carried out by entities falling into three major groups:

- Higher education institutions supervised by the Ministry of Education

- Public research and technology centers, most of which are supervised by the General Secretariat for Research and Technology, Ministry of Development

- Private sector organizations

Although the Ministry of Education exerts great influence on the national research system due to the large number of universities in the total R\&D effort, the only body that has an explicit research and technology policy in Greece is the General

$12 \mathrm{ftp}$ //ftp.cordis.europa.eu/pub/greece/docs/rdingreece_gsrt_2003_en.pdf, accessed 15 August 2008. 
Secretariat for Research and Technology (GSRT) of the Ministry of Development. Its share in the government financing of $R \& D$ is about one-third of the overall budget. $R \& D$ expenditure of the Higher Education Institutes presents a steady upward trend. As a percentage of GDP it has been steadily increasing since 1993, reaching $0.33 \%$ in 1999 (R\&D, 2003). Greek research may seem to be constantly improving, looking at the increase of gross expenditures in $R \& D$ and by increasing trends in R\&D personnel.

\subsubsection{European Elements}

Greece, as a member of the European Union, also has to respond to the quality assurance initiatives by following European directives on recognition of degrees. On June 19, 1999, Ministers responsible for higher education from 29 European countries signed the Bologna Declaration. They agreed on important joint objectives for the development of a coherent and cohesive European Higher Education Area (EHEA) by 2010 (Bologna 1999). Apart from some regulated professions, most of the role of the EU Directives has been taken over by the recognition elements included in the Bologna Process, such as the Diploma Supplement and the implementation of national qualification frameworks. However, much of the influence of the Bologna Process only started to appear at both the European and the national Greek levels after the end of the period studied here, i.e. after 2006. Yet already at that time, the Secretary for Higher Education, Professor Kyriazis (2006) observed that "there has been great effort in Hellas during the last two years to modernize the higher education sector so that it can meet the contemporary needs of society and the economy, both at national and European levels". The establishment of the State International Hellenic University in Thessaloniki, he said, constituted the first systemic step towards the opening of the Greek Higher Education abroad, for which the responsibility lay with the Greek state itself. This new university was opened to anyone from any country in the world and in particular to the youth of the neighbouring region (South-East Europe).

In the Berlin communiqué of 19 September 2003, the Ministers of the Bologna Process signatory states put quality 'at the heart' of the process and required all states to have quality assurance procedures in place by 2005. The Ministers also asked ENQA and the other members of the 'E4' to develop guidelines for national quality assurance procedures. In Bergen, in May 2005, the European Standards and Guidelines for Quality Assurance were agreed by the Ministers at their next followup conference. Besides, the monitoring of progress among the signatory countries was stepped up by the 'stocktaking' exercise, which first of all demanded that all participant countries produce National Reports to inform the stocktaking 
working group of their country's progress. The Greek National Report claimed that the framework for operating a National System for Quality Assurance in Higher Education was under consultation before the Greek Parliament. This framework is now a Law (ref: Law 3374/2005). Kyriazis (2006, p. 6) also stated that Greece took part in the European Union's Lisbon Strategy to develop the knowledge-economy and social prosperity and cohesion. Within this framework, Greece modernized its whole educational system.

Moreover, Greece is the coordinating country of the Education Group within a regional cooperation, the Black Sea Economic Cooperation, in which twelve countries participate, including Greece (Kyriazis, 2006, p. 5).

\subsubsection{Greek National System for Quality Assurance in Higher Education}

In Greece during 2005, the implementation of quality assurance was in its early phases. The Greek Government established a national system for quality assurance in higher education (Greek Law 3374/2005 - Greek Government gazette Issues (FEK) 189/August 2, 2005). According to this Law, which will play a role in chapter 6 as well, the national quality assurance system is composed of two levels:

1. internal assessments and

2. external evaluation and review schemes

According to the law, universities are encouraged to set up their own internal quality assurance mechanisms for its educational, administrative, and research functions to provide a sound of basis for external evaluation. The aim is to effectively combine institutional autonomy and accountability within the national quality regulations framework. Furthermore, teaching staff, administration personnel, and students are viewed as the main participants and contributors to this process.

Law 3374/2005 includes articles which address the following aspects:

- Scope, content, and objective of the evaluation

- Evaluation process

- Evaluation criteria and indicators

- Internal evaluation

- Internal evaluation process

- Internal evaluation report

- External evaluation

- External evaluation committee

- External evaluation report

- Committee for the Assurance Quality in Higher Education (A.D.I.P.) 
- $\quad$ Members and structure of A.D.I.P.

- Administrative and scientific support of A.D.I.P.

According to this Law, a single national agency in charge of quality assurance is an essential feature of the Greek higher education system. It aims at quality improvement through external evaluation. Notably, the Hellenic Quality Assurance Agency for Higher Education-APXH $\triangle \mathrm{IA} \Sigma \Phi A \Lambda \mathrm{I} \Sigma \mathrm{H} \Sigma$ ПOIOTHTA $\Sigma$ $\mathrm{AN} \Omega T A T H \Sigma$ EKПAI $\triangle \mathrm{E} \Upsilon \Sigma \mathrm{H} \Sigma(\mathrm{A} \triangle \mathrm{I} \Pi)$-website appeared for the first time on March $2007^{13}$.

The quality assurance system and assessment established in Greece is intended to support higher education institutions in their efforts to continuously improve their quality and to advise the government on the necessary actions and policies to be taken to that end. At the same time, it aims to improve transparency, comparability, and accountability of the Greek higher education system. It does not contain accreditation characteristics, nor does it rank or grade the Greek higher education institutions. Additionally, it does not have authority to impose penalties or reward schemes.

\subsubsection{Technological Elements}

The focus of this section is to provide a look at a specific repertoire of technological tools in Greece, namely the managerial culture in general and the issue of quality management more specifically. Quality management practices from outside the university setting, e.g. from industry and business fields, may influence universities. As noted earlier, such technology is salient in the operation of organizations; if it is thought to be significant, other organizations will seek to buy it, copy it, or further extend the previous development. Thus, it is important to scan the managerial technology within public and private sectors with a special look at quality management initiatives and to examine how these appeared in Greece.

Bourantas et al. (1990) sought to define the idiosyncrasy of management in Greece and found it empirically in a confirmation of "the arguments advanced by other researchers on the existence of ... culture gap" (p. 274). The authors commented that the existence of the culture gap could explain to a degree the paradoxical finding of Veiga et al. (1987) that "in Greece, although there is a culture which has a high regard for collectivism and group well-being, Greek managers have a lower propensity 'to give up control' than American managers"' (p. 275). The

13 Detailed information regarding the National System for Quality Assurance in Higher Education (NSQAHE) is available at www.qhaa.gr 
authors also stated that "Greek managers tend to behave in a power-oriented manner much more than do U.S. managers". Bourantas et al. (1990, p. 275) believed that this behavior "may well be a result of the culture gap which creates a feeling of an 'outgroup setting', in which, according to the work of Triandis et al. (1968), Greeks tend to be extremely competitive, hostile, and suspicious and engaged in defensive responses to authority figures". The authors wrote that "it is fairly obvious that this study confirms the existence of certain peculiarities of Greek management which were detected over a quarter of a century ago". Their conclusion was that "the Greeks must learn the European management styles by first imitating the competitors and finally incorporating these management techniques and styles into their own styles" (p. 276). For Bourantas et al. (1990, p. 276) learning implies education. "It is thus strongly implied that Greek institutions of higher education begin to re-evaluate their traditional overwhelming emphasis on the legal and accounting aspects of business and the political features of the external environment". The authors suggested that "business education standards of Western Europe must be introduced into businesses school's curricula" (p. 276).

Bourantas and Papadakis (1996) also were interested in the Greek management context. They found that Greece has "a very large public sector, which is often uncompetitive, and its huge deficit is a major problem to the whole Greek economy" (p. 2). The authors discussed the gap between cultures and management practices of Greek-owned enterprises and those of subsidiaries of multinationals operating in Greece. The authors found that among the main forces inhibiting evolution was the "administrative heritage" - the lack of a strong and differentiated Greek management culture, the political intervention, and the weak economy of Greece in comparison to that of other EU countries. Yet, the authors believed that "the change forces are so strong that it is highly unlikely that any negative forces will inhibit the modernization of Greek management". Bourantas and Papadakis found that their analysis reinforced their basic argument: while still underdeveloped, Greek management is in a fast-track to modernization and professionalism. At the same time, they stated, "what is more discouraging is that most of the published studies suffer from lack of coordination. Thus, they are of limited value in accumulating knowledge, and using it to describe and explain management" (p. 12). Bourantas and Papadakis mentioned that the Makridakis' et al. (1997) survey was a notable exception.

Makridakis et al. (1997) compared the characteristics of the Greek CEOs with the characteristics of Western European and American CEOs. In their survey, several questions dealt with the usage of the various management tools and techniques. They found that Greek firms used such tools and techniques, but not as much as 
their rivals abroad. Also, they discovered that two important tools were utilized much less in Greece than abroad. The first, total quality management, was used on average by only $35 \%$ of Greek firms, compared to $73 \%$ of firms abroad. The second, business process re-engineering, was used on average by $42 \%$ of Greek firms, versus $71 \%$ of firms abroad. Makridakis et al. (1997) discovered that $19 \%$ of the Greek CEOs mentioned technology as critical, while the percentage of Japanese CEOs with the same response was $49 \%$. The authors also took into account issues related to the Greek environment. They wrote that Greece combines some of the best and the worst characteristics of modern life. For instance, they mentioned that the life expectancy in Greece is one of the highest in the word, while the suicide rate is the lowest. At the same time, there are more days lost in strikes in Greece than in most other countries in the world, despite having one of the world's most centralized bureaucratic administrations, second only to China's. Furthermore, Makridakis and his associates (1997, p. 394) stated that "in Greece, any kind of planning is difficult, while long-term strategy is practically impossible. The joke is that long-term planning involves next week; medium-term, tomorrow; and short-term, the next hour". Two things make planning difficult. First, laws and regulations change frequently and without warning. Changes occur not only when government is taken over by another political party, but also when personnel changes occur within the same party, e.g. when a new minister from the same party takes over. The second reason is simply that more unexpected things seem to happen in Greece than in other advanced countries. The authors found that the combination of these two interrelated factors, frequent changes and unexpected events, forces Greek managers to concentrate on the short term. The authors' view was that the "short-termism" was the worst characteristic of Greek management and also of Greek governments. The authors observed that this fact was true (at least in 1996) for the great majority of Greek firms and undoubtedly for all Greek governments, which without exception concentrated practically all their attention on solving the "crisis of the day" instead of formulating and implementing a long-term strategy to eliminate or reduce such crises.

Now our focus shifts to a study on another quality management practice: ISO 9000. Tsiotras and Gotzamani (1996) claimed that in Greek industry, companies first began to develop quality assurance systems in the 1990s. In the beginning, the propagation of quality assurance systems in Greece was rather slow, mainly due to the lack of adequate information about them, but later the growth became exponential. Tsiotras and Gotzmani found this was a domino effect, and the reason was the inclusion of ISO 9000 certification within European procedures for the certification of industrial products and the demands of already certified companies to their suppliers. In a similar vein, Lipovatz et al. (1998) stated that 
“the implementation of QAS [quality assurance system] in Greece began quite recently, after the year 1990. In 1993 only 30 companies possessed a certificate in accordance with ISO 9000, but the number of certificates has grown rapidly to 222 in 1995". The authors also observed that despite the highly increased rates, the total number of certificates remained low, taking into consideration that at the same time Ireland possessed 1,617, and Denmark 1,314. Finally, the authors observed that the most important reason for the introduction of quality assurance systems in Greek enterprises was because of the external (i.e., the adjustment to the demands of the international and/or domestic market) and not the internal impact of certification (i.e., the improvement of the organizational structure and the reduction of production costs) (p. 548). The authors' results demonstrated the strength of mimetic pressure.

Quality management issues in the Greek construction industry and in hospitals provide additional considerations related to the technological repertoire in this university study. Zantanidis and Tsiotras (1998) examined quality and the structure of a quality assurance system in the construction industry, a very important business sector in Greece. They found confusion among middle management in the construction industry regarding the meaning of quality and its real critical characteristics. Only $40 \%$ of the firms responded that their quality control efforts were systematic. The authors stated that among the main elements of the quality assurance system were the quality assurance manual, quality procedures, documentation, and quality audits. These elements appeared to be absent in most of the firms examined. Their results suggested that the barriers to effective introduction and implementation of quality assurance principles in the Greek construction industry are manifold and complex.

Theodorakoglou and Tsiotras (2000) investigated the organized and scientific implementation of quality management in Greek public hospitals. They found implementation of quality management systems in Greek public hospitals to be rare and stressed the need for the introduction of quality management into Greek healthcare. They also observed that leaders, who play the most basic role in supporting quality programs, lacked basic education in quality issues. The authors found that in most cases, the implementation of quality programs depended on employees' initiatives and did not stem from an organized, central plan or from a formal quality policy.

One subfield in the TQM literature focused on the management of human resources (HRM) (Dale et al., 1997). Papalexandris and Chalikias (2002), are among the few who researched HRM in Greece and who investigated the progress in the practice of HRM in Greek organizations by comparing results 
from 1992 to those of a 1999 survey that focused on various core HRM functions (i.e., training and development, performance management, and employee communications). They compared these developments to the overall European scene and found a slower pace in improvements. In their findings, they reported that Greece had a great need for training in all specializations. In particular, IT, training in new technologies, and training in customer service and quality management were most required, which was in line with the EU trend to develop competencies in those specializations. The authors also commented on performance appraisals, stating that "in Greece, appraisal is based more than in the EU on the next level superior, while employee participation in the procedure is still considerably lower, something quite normal given the somehow negative attitude of employees towards performance appraisal" (p. 345).

In addition, Spathis et al. (2002) investigated the quality of services provided by banks based on customers' perceptions using the banking service quality model. The main objective of their study was to detect customers' perceptions of service quality in private and state-owned banks in Greece and to examine the quality environment in which profitability flourishes. Their findings suggested that the perception of the profile of services was higher in the private sector than in the public sector on all factors. Their results with respect to customer perceptions suggested that the service offered by banks in the private sector have a more favorable influence on the actual perceptions of quality than in banks in the public sector. Their evidence showed that public banks did not manage the factors influencing quality as well as private sector banks. The authors noted that if service quality matters, customers may drift toward the private sector. Myloni et al. (2004) stated that Greece, together with France and Italy (Koopman et al., 1999) belongs to a separate cultural cluster in Europe distinct from the North and West. Empirical results of Myloni et al. indicated that HRM practices in Greek firms reflected national culture to a large extent. The authors quoted Laurent (1986) and Schneider (1989), who stated that, as most quality management practices, HRM practices are grounded in cultural beliefs that reflect the basic assumptions and values of the national culture in which organizations are embedded (Myloni et al., 2004, p. 62).

The above reviews present the organization-technological environment in which Greek universities operated. Several steps had been made in private and public sectors to adopt quality management initiatives. Dervitsiotis (1999) stated that most initiatives at the end of the twentieth century had been undertaken by associations of business firms and technical professionals, and by universities through training managers in the principles of total quality management. He also stated that "the Prime Minister Simitis declared that 1998 will be the year of 
quality' in all sectors of the economy". Dervitsiotis also pointed out that the University of Piraeus became the first in Greece accredited by the Ministry of Education in 1994 to offer a full graduate program in Total Quality Management (TQM). These days, many universities in Greece offer courses and programs related to quality management. For instance, the University of Macedonia started to offer TQM studies specifically for public servants in September 2008.

\subsubsection{Sociocultural Elements}

The sociocultural environment includes population demographics, explosion of knowledge, rates of birth, changes in human trends, ethnic diversity, and income distribution. The university is a symbol of "public benefit" and it is important to the operation of the society in Greece. Access to a Greek university is a crucial characteristic in its physiognomy, as is diversity of the population ${ }^{14}$. This part addresses access to higher education and trends, Greek student body's characteristics, international students, life-long learning, and the social role of the university.

\subsubsection{Access and Student Numbers}

Access to higher education in Greece is a dream for almost every adolescent and that access is very competitive. At the same time, access to higher education in Greece is dictated by numerous conditions. Every year, the number of new undergraduates allotted to each department of each university is determined by the Greek Ministry of Educational and Religious Affairs (YPEPTH), as it was named in the period studied. Legislation stipulated that universities submit proposals; however, the Ministry often did not take these proposals seriously, according to newspapers articles and rectors' announcements.

Furthermore, admission to undergraduate higher education institutions (both universities and technological educational institutes) follows a process of national examinations. The success of candidates in these examinations and their access to a specific department depends upon a combination of the candidates' scores on the examinations, their preferences for a department, and the number of places allotted. The entry examinations lead to an annual social agony. Psacharopoulos (2003) underscored that agony stating "perhaps nowhere else in the world does annual tertiary education entry examination immobilize the nation". The exams

\footnotetext{
14 www.financial-directory.gr, Greece in Figures, 2006.
} 
make newspaper headlines, describing the agony potential undergraduate students and their families go through until (or if) a university place is secured.

At the graduate level however, the universities have autonomy in their selection of graduate students. In general, selection of graduate students is based on a combination of factors, including grades, recommendation letters, and knowledge of a foreign language. Table 5-3 presents the number of students and graduates of Greek universities.

Table 5-3 Number of students and graduates of universities

\begin{tabular}{ccccc}
\hline \multirow{2}{*}{$\begin{array}{c}\text { Academic } \\
\text { year }\end{array}$} & Students & $\begin{array}{c}\text { Graduates with } \\
1 \text { st degree }\end{array}$ & $\begin{array}{c}\text { Postgraduates } \\
\text { (Master's) }\end{array}$ & Doctoral degree \\
$1996-97$ & 244,970 & 22,770 & 846 & 740 \\
$1997-98$ & 253,915 & 21,309 & 1,555 & 728 \\
$1998-99$ & 266,103 & 21,154 & 1,354 & 796 \\
$1999-00$ & 276,902 & 22,784 & 2,275 & 1,049 \\
$2001-02$ & 325,001 & 24,391 & 3,403 & 1,154 \\
$2002-03$ & 342,640 & 27,547 & 3,765 & 1,237 \\
$2003-04$ & 352,936 & 29,477 & 5,012 & 1,296 \\
\hline
\end{tabular}

Source: www.Euridyce.org

The Greek higher education statistics only recognize and track full-time students. Considering the effects of the more than $40 \%$ growth of student numbers illustrated in the table, Papadimitriou $(2009$, p. 11) observed, "in the university, professors are required to develop students' critical thinking skills, by teaching case studies, scenarios and by actively participating in the classroom. That is not an easy task considering that class sizes are large and the student participation is low and voluntary". The above tables, along with the following readings, illustrate Greek modern society and its "educational paradox". The majority of Greek secondary school graduates, and especially their families, desire a higher education degree without taking into consideration the cost and "added value" from this degree. Psacharopoulos (2003, p. 125) observed that Greece has an "insatiable demand" for education. The dominant explanation of such a phenomenon was that in a period of massive rural-urban migration, parents saw education as a means of escaping from the village to facilitate social mobility. He also stated that the competitiveness for university entry has given rise to the proliferation of cramming schools for university exam preparation called "frontirstiria". Each year, over one billion Euros were spent on preparatory courses at these cramming schools, which he called "misallocation of resources". 
This amount is more than what the state spent on secondary schools and such resources could be used for improving the quality of instruction if the money flowed through public hands.

The social demand for higher education in Greece remains high, and this trend is attributed to the still strong traditional reputation of the universities and to the social prestige that university degrees ensure for the graduate, regardless of the high possibility of remaining unemployed, underemployed, or employed in other sectors for a long period. Furthermore, top executives in higher education emphasized that this paradox should not lead to a conscious distinction between the social and personal right for higher education studies on the one hand and the personal right for employment on the other.

The concept of "degree hunting" is for the family economic "bloodletting". Greek households invest in their children's studies without any guarantee for this "investment". This is apparent in the high level of unemployment of young people and the irrelevance their degrees have to the job market (OECD, 2006). An article in the Greek newspaper Eletherotipia stated in April 18, 2007, that Greece showed the highest youth unemployment rate among the 27 members of the EU in youth unemployment. The unemployment rate was $7 \%$ among graduates from higher education in Greece. According to the newspaper Kerdos (Kerdos.gr, 1-62006), "The Greeks work in different jobs than they studied". The article presented a survey from March-May 2006 (population: 2,317, age: 18-35, 58\% female, $42 \%$ male) showing that more than $67 \%$ of new graduates in the workforce were in a different job than the field of their studies. The majority of respondents (53\%) did not believe that a bachelor's degree could help them find a job, and moreover, $51 \%$ stated that there were not enough job opportunities related to their studies or degree, and they were not satisfied with the connection of their studies with the work environment.

At the same time, Greece is fourth in the absolute number of students studying abroad. But if one takes into account the size of the population, Greece is the undisputable world leader (Psacharopoulos, 2003). Thus, OECD data had found that $40,654^{15}$ Greek students studied abroad around the time covered by my study (OECD, 2006). Greece's population is about 11 million ${ }^{16}$, yet it had more students abroad $(0,36 \%)$ than Germany $(0,09 \%)$, with a population of 82 million $(78,242$ students abroad, according to the OECD, 2006).

15 OECD, 2006, Education at a Glance, Table C3.7

${ }^{16}$ OECD, 2009, Education at a Glance, Table X2.2, (reference period 2006) 
OECD (2006) statistics show the small percentage of international students in Greek public universities. Nevertheless, Table 5-4 illustrates the international population in Greek universities by country of origin for the academic year 20002001 and 2004-2005 (Papadimitriou, 2005). Greek universities draw most students from neighboring countries (Albania, Bulgaria). Compared with other EU countries, it is remarkable that by far most foreign students hail from non-EU countries (but not China or Arabia).

Table 5-4 International students in Greek universities

\begin{tabular}{lcc}
\hline \multicolumn{1}{c}{ Country } & $\begin{array}{c}\text { Academic Year } \\
\mathbf{2 0 0 0 - 2 0 0 1}\end{array}$ & $\begin{array}{c}\text { Academic Year } \\
\mathbf{2 0 0 4 - 2 0 0 5}\end{array}$ \\
\hline Albania & 49 & 77 \\
Bulgaria & 59 & 68 \\
Egypt & 4 & 12 \\
Ethiopia & 10 & 34 \\
Georgia & 14 & 19 \\
Pakistan & 55 & 1 \\
Poland & 7 & 14 \\
Rumania & 16 & 13 \\
Russia & 15 & 33 \\
Serbia & 5 & 13 \\
Sudan & 13 & 2 \\
Syria & 22 & 29 \\
Ukraine & 12 & 40 \\
$\ldots \ldots \ldots . .$. & $\ldots \ldots$ & $\ldots \ldots$ \\
\hline Total & $\mathbf{3 8 6}$ & $\mathbf{4 4 6}$ \\
\hline Source: A. Papadimitriou (2005). Other countries: less than 10 \\
per country in both years. & &
\end{tabular}

Kokosalakis (2001) characterized life-long learning as a result of wider national policies of educational reform in the context of socio-economic change. $\mathrm{He}$ pointed out that the use of the term "life-long learning" in Greece is very recent. Research undertaken by the European Centre for the Development of Vocational Training in 2003 revealed that Greece placed second lowest among all EU countries in response to the question: "Is life-long education important?" The Hellenic Open University, established in 1992, was created to address this type of higher education. The number of applicants to Open University in 2003-2004 was 52,346 for 5,000 openings, and this number increased in 2006-2007 to 61,560 for 6,660 available openings. We can say that the number of applicants followed the same trends as those of traditional university students. Access to the Open 
University is for students who work and are over 25 years old. Other universities are also encouraged by law to offer life-long studies and programs.

\subsubsection{Universities and Communities}

Universities in Greece have a special social role. Every Greek university operates in the same fashion but in a different regional environment, which affects its organization and performance. Universities are also a part of the urban life. The social role of the university is demonstrated in its organization and in its internal operations report. Every university confronts this issue to a different degree, including the Rectorate, because it is considered in the strategy of every university. The following box presents an example of this trend in one university; similar issues are apparent in many other universities in Greece (Papadimitriou, 2004).

\section{City and University}

The University $X$ has a special social role. For many centuries universities were "closed temples" of sciences. Today, they are the nuclei of education and of high-level research, they are interwoven with society, and they operate for the benefit of the citizens. At the same time, remarkable changes in teaching methods are taking place, based on the use of computers and the internet, in digital libraries, distance learning and on the opportunity provided for students, teachers, and administrative staff to move around the world. One could say that the University X is a "city" within the city.

This university has substantial economic, educational, cultural, and political influences throughout the city. It is a big spender and annually allots enormous amounts of money for goods and services required by the institution. Add the money spent by students (houses rental, food, clothing, theatre, movies, and festivals), their families, faculty, staff, alumni, visitors, and local businesses on behalf of the university and one can see the impact the university has on the economic status of the city. This difference is extremely apparent in the summer time when it is an "empty" city, without students.

The university offers educational services to the local communities. Delivery of degree programs, "Open University" for the citizens offering non-credit classes, seminars, and courses on a wide variety of topics, and formal training services are exceptionally valuable to the quality of life of city residents. Also, the university's museum, performing arts facilities, and other cultural attractions are well-positioned to support the school in is educational efforts. Its facilities are affordable and are supported with faculty, students, and staff. The university's hospitals also offer very high quality health services to the community. Partnerships between students and the city are very common. These partnerships can involve internships, part-time employment, volunteer programs, and participation in various practical training programs as internships.

In addition, Professor Massalas, President of the University of Western Macedonia (Personal communication, June 2006), emphasized the role of the Greek university and the learned society: 
The main contribution of a regional university to the local community is, or should be, the dissemination of an optimistic environment, a culture of new technologies as well as a culture of quality.

At the same time, he was critical of how Greek universities had taken up this local or regional role:

The Greek universities, after remaining comparatively isolated for a very long period, both in relation to society and to the rest of the world, with funding (restricted) guaranteed and a status protected by respect for their autonomy, have gone through the second half of the $20^{\text {th }}$ century without really calling into question the role or the nature of what they should be contributing to society. The changes they are undergoing today and which have intensified over the past fifteen years prompt the fundamental question: can the Greek universities, as they are organized now, hope in the future to retain their place in society, in Europe and in the world? In my opinion, if the present tendency continues, Greek universities may be more or less out of business. It is high time to start thinking differently and to act on this.

One source of possible changes could be the pressure from students. Students are very strongly and politically organized, as discussed above.

\subsection{Internal Dynamics}

Along with external environmental elements, it is also crucial to highlight the internal elements (university characteristics) that affect the organizational life and performance; therefore, this section deals with the internal organizational dynamics wherein Greek public universities operate.

As previously discussed in section 5.1.2.1, Article 16 in the Greek Constitution of 1975 provides for higher education, stating that arts and sciences, research, and teaching are free and their development and promotion constitute a state obligation. Academic freedom and the freedom to teach do not override the duty to obey the Constitution.

In addition to the Constitutional provision, frame-law $1268 / 82$ provides for the academic freedom of teaching and research. Law 2083/92, Article 5 also requires the drafting of the Internal Operation Bylaw describing the function of the university. The Senate of the university, in its meeting, has to discuss and approve such a Bylaw. This meeting later had to be published in the Official Journal of the Government. This Bylaw governs matters such as the organization 
of the academic and administrative functions of the university and the process of electing the members of the Senate. It also sets the rules for the function of committees and councils, for the rights and duties of teaching staff and all other personnel, the rights and duties of the students, disciplinary procedures; undergraduate and graduate studies and certain other organizational matters.

\subsubsection{Academic Personnel and Departments}

The final authority for setting up new academic units (universities, schools, departments, divisions) and for renaming, merging, splitting, or closing down existing academic units belongs to the YPEPTH. During the academic year 20052006, there were 266 departments in Greek universities. The total faculty population consisted of 14.439 members, 9.782 male and 4.657 female $^{17}$.

The existing frame-law for the Greek universities specifies four distinct levels of academic structure inside the university. These four levels are as follows:

- Institution

- School

- Department

- Division

The basic unit in the university's inner structure is the department. Departments correspond to an area of knowledge (discipline). They award degrees and they are also the academic units to which the positions of the main teaching personnel belong. The Departments have full autonomy in the election of their academic staff at all levels but the YPEPTH determines the number of positions to be filled each year and checks the legality of the staff selection processes.

Departments are divided into divisions or sectors corresponding to smaller and distinct parts of the major scientific disciplines of the department, provided the department's discipline area is adequately broad and the department's faculty is sufficiently large.

The teaching and research activities of a department or a division/sector may be grouped and concentrated in even smaller operational units, the so-called laboratories (or clinics, in the case of medicine).

Departments covering related disciplines areas may constitute a School, which has mainly coordinating responsibilities to its departments.

${ }^{17}$ Ministry of Education website, www.ypepth.gr, accessed 10 July 2008. 
Not all universities in Greece are organized in similar ways; some do not have schools or research institutes or departments.

\section{Personnel}

According to Greek legislation, university personnel consist of the following three major categories:

1. the academic staff

2. the technical and laboratory staff

3. the administrative staff.

More specifically, the academic staff consists of the following categories:

1. the main teaching staff faculty (professor, associate professor, assistant professor, and lecturer) all hold Ph.D.s. Faculty is comprised of persons who hold doctoral degrees and are members of the socalled Teaching and Research Faculty

2. adjunct and visiting teaching staff (who normally have a Ph.D. but collaborate with the university on a temporary and contractual basis)

3. special teaching staff and research associates (mostly without a Ph.D. degree, teaching special subjects)

Only the members of the two upper levels, professors and associate professors, are elected in permanent (tenure) positions. To safeguard academic freedom, university academic staff (faculty members) are public functionaries who may not be dismissed before the expiry of the term of their employment, save under very special circumstances. The rest of the staff are also public servants. The YPEPTH directly controls the salaries of the permanent personnel.

\subsubsection{Decision-making}

As noted previously, frame-law 1268 established the values and the principles of democracy, collective participation, accountability, and transparency for Greek universities. These values and principles reflect the regulations concerning decision-making and leadership.

Each academic unit has its own leadership and decision-making structure. There is a hierarchy among the four levels of academic structure concerning leadership and decision-making, with the institution at the top and the division at the bottom. Within this hierarchical structure, the role and responsibilities of the school are limited to coordination of the departments. The structure of leadership 
and decision-making in the above four levels of academic structure are depicted in Table 5-5:

Table 5-5 The structure of leadership and decision-making

\begin{tabular}{|c|c|c|c|c|}
\hline Authority & \multicolumn{4}{|c|}{ Academic level } \\
\hline $\begin{array}{c}\text { Governance/ } \\
\text { Leadership }\end{array}$ & $\begin{array}{c}\text { Institution } \\
\text { Vector }+\end{array}$ & School & Department & Division \\
\hline Decision-making & Senate & $\begin{array}{c}\text { Gead } \\
\text { Assembly } \\
\text { (+Deputy Head) }\end{array}$ & Director \\
\hline Decision-making & Rector's Board & $\begin{array}{c}\text { General } \\
\text { Assembly } \\
\text { Board }\end{array}$ & $\begin{array}{c}\text { Governing } \\
\text { Council }\end{array}$ & Assembly \\
\hline Executive & $\begin{array}{c}\text { Rectorate } \\
\text { Council }\end{array}$ & $\begin{array}{c}\text { Dean's } \\
\text { Board }\end{array}$ & $\begin{array}{c}\text { Governing } \\
\text { Council }\end{array}$ & \\
\hline
\end{tabular}

\subsubsection{Decisions Regarding Teaching and Research}

The following levels of authority regarding teaching and research in universities are distinguished.

- Individual level: each faculty member is responsible for and decides upon the exact content and teaching method of his courses and the basic or applied research he conducts.

- Division level: decisions concerning the undergraduate and graduate curricula and new faculty positions, according to the teaching and research needs and priorities of the division, are subject to approval by the Department's Assembly.

- Department level: decisions concerning the undergraduate and graduate curricula and new faculty positions, according to the teaching and research needs and priorities of the department

Apart from the line hierarchy, other entities with authority in the university include:

- Research Committee level: admittance and financial administration of research projects and funding of specific research projects from its income (see Section, 5.1.2.1)

- Company for the Management of the University Property level: decisions about investments and university assets management, funding of 
specific research projects, or other university related initiatives (see Section 5.1.2.1)

- Research Institute level: decisions about research directions and priorities of the institute (see Section 5.1.2.1)

\subsubsection{Leadership Positions and Senate}

The Rector and the two or three Vice-Rectors, depending on the size of the university, are elected as a Rectorate for a three-year mandate by an electoral body consisting of: a) all the faculty members, b) representatives of undergraduate students, who number $80 \%$ of the population of category a, and c) representatives of all the other categories of personnel who number $25 \%$ of the population of category a. In this way, none of the groups has a majority, but no group has veto power, either.

The Senate of the university consists of the following members: a) the Rector and the two or three Vice-Rectors, b) the deans of all schools and the heads of all the departments, c) one representative of the undergraduate students, d) two representatives of the postgraduate students of the institution, e) four representatives of all the other categories of personnel, and six to eight representatives of the faculty belonging to levels other than the level of professor.

The Rector's Board of the university consists of the Rector, the two or three ViceRectors, one representative of the students, and the Registrar (Secretary General) of the university. For a better view, Table 5-6 presents the main decision-making bodies in universities and the main responsibilities assigned to them by law.

Table 5-6 Decision-making bodies and major decisions

\begin{tabular}{ll}
\hline \multicolumn{1}{c}{ Body } & \multicolumn{1}{c}{ Major Decisions } \\
\hline Senate & - determines the education and research policies \\
& - initiates contacts with stakeholders (staff, students, society, \\
etc.) & proposes the establishment of new departments to the \\
& Ministry of Education \\
- approves the university budget \\
- designates committees for specific subjects \\
- approves university asset management \\
- proposes the university budget to the Senate \\
- proposes new teaching and research positions to the \\
Rector's Board & Ministry of Education \\
\hline
\end{tabular}




\begin{tabular}{ll}
\hline Rector & - directs the administration operations \\
Dean's Board & - decides budget allocation to the departments of the school \\
Department Assembly & - determines the education and research policies for the \\
& department \\
& - allocates the budget to the divisions and laboratories \\
Research Committee & - responsible for financial management of funded research \\
(headed by the Vice- & projects \\
Rector of Academic & - proposes the research policy of the university \\
Affairs and Staff) & - proposes the allocation of its income \\
Company for the & - responsible for financial management of university's \\
Management of the & property \\
University Property & - proposes the allocation of its income \\
(headed by the Rector) &
\end{tabular}

\subsection{Summary and Conclusion}

This chapter presented a general overview of Greek universities' external and internal environments as background information and to set the stage for the empirical studies that follow.

The first section provided a brief introduction and historical setting of Greek higher education where the concerns were about university characteristics. The Greek higher education system in general began with the first university, founded in 1837. Since then, they have grown into 21 public universities, which are divided in two categories: 15 old (founded before 1982) and six new after 1982. Another division defines seven monothematic and 14 multidisciplinary universities. The universities are located across Greece and they are defined as urban, if they belong to the 10 universities in Athens and Thessaloniki, the remaining 11 are called peripheral universities. Greek higher education institutions cannot be discussed further without discussing the primary overriding governmental, legal, and political control, which influences all major decisions in the Greek higher education institutions. The public universities are on average for $97 \%$ state funded and that is a significant factor in the external environment of all Greek higher education institutions. Private universities were not recognized in Greece until 2010. Laws define not only the mission of all universities, but they also deeply pervade daily routine practice. Nevertheless, politics are relevant as well, not the least of which occur through the protest power of students. The number of students has risen rapidly over the period studied (over $40 \%$ in the last 6 years). Additional external elements in the environment stem from the European level (the Bologna Process, and quality 
assurance practices). Access to higher education, current trends and characteristics of the student body, and the social role of the university were included in the sociocultural elements discussed in this chapter. Then, a discussion about application of quality management and managerial culture in Greece was provided to scan the managerial technological environment. Within the higher education institutions, the internal structure focuses on the department level where most of the operational decisions are made; furthermore, various academic organizations influence those decisions. These organizations provided the internal environment as described in the second section. Additional factors that influence the internal environment are comprised of the socioeconomic composition of each of the universities at all levels and the technological developments which engage the partnership of education and research; this partnership realizes a significant monetary gain to the participating universities.

As organizations, universities have been characterized as complex. The legal/political context in which Greek universities operate describes the degree of complexity under which these universities exist. The legalistic tradition indicates a system subject to a plethora of laws and regulations established by a highly politicized state bureaucracy. Based on the above, and since all Greek universities have been awarded permission to operate by the Ministry of Education, the Ministry is functionally equivalent to an accreditation agency that generally defines standards for the operation of Greek universities.

In recent years, the role of experts in European higher education has increased and new research organizations and centers have been created. However, higher education studies still are limited. Perhaps in the future, the attentiveness of new roles for the academic and/or political elite might include both quantitative and qualitative studies that can adequately document whether the Greek and the political system have been fundamentally modernized and Europeanized. As awareness of quality management and the need for policy reforms grows stronger, future decisions will need to be made with evidence based on research.

As previously noted, the Greek Constitution provides free education for all, and Greek society ranks a university degree very highly. Families spend large amounts to prepare their children for access to higher education either in Greek universities or abroad. However, many Greeks, upon their graduation, work in different jobs than those for which they studied. Such an observation might engender questions about the efficiency of the students' preparations. At issue here is that many students do not study what they initially wanted to study, except for the highest-scoring students who are able to enter the department of their first choice. As for the remainder of the student applicants, their first choices 
are filtered by the electronic system of the Ministry of Education and students are placed in departments based on space available, not necessarily upon the students' first choices.

We find that the vast majority of students are traditional, young, fresh out of secondary schools without job experience, who benefit from the State in terms of books, meals, and (for low-income students) free dormitories; meanwhile none of the undergraduate students pay tuition fees. Greek universities have very few international students.

The sociocultural scanning did not reveal any evidence that quality assurance and quality standards were valued or requested by students' families. However, Greece as an EU member, follows the European higher education developments; therefore, a slow but important transformation is taking place. The impact of European integration in Greece deals with the adoption of ECTS, the development of the International University, and regarding quality assurance systems Greece was a most recent adopter. Only as recent as 2005, the quality assurance law appeared, and practically all universities were required to submit their first self-assessment reports from the academic year 2007-2008, onwards.

The Greek Government stated that the country's European perspectives need to be based on the introduction of competitiveness in all spheres of economic, educational, and social life. However, entrepreneurialism and the adoption of modern ideas in education create fear of the unknown, scepticism, and sometimes strong cultural opposition; the over-riding idea that education is a public good has strong advocates, which comes from deeply seated roots in Greek's political culture.

As discussed in this chapter, the attitudes and Greek culture, although not diametrically opposed to the concept of quality management, require significant adaptation to this managerial technology in both the private and public sectors (including the universities).

This scan of internal and external environmental factors indicates a very weak base for the adoption of quality management in higher education. Therefore, in the empirical studies that follow, I used the isomorphism perspective to explain organizational change. There are other voices who noted that "today the Greek university is a behemoth, unable to respond to the changing social environment and demands" (Keridis \& Sfatos, 1998, p. 174). Nevertheless, a former Minister of Education noted: "we have a difficult and a rocky road ahead of us no doubt, but also a challenging one. We are willing and able to meet the challenges" 
(Papadendreou, 1998, p. 25). Those opposing voices reflect the difficulties that this study encountered in its attempts to explain the degree of adoption of quality management in Greek higher education. 


\section{Media Coverage of Quality Assurance}

\subsection{Introduction}

The Bologna Declaration, from a policy and juridical perspective, could be viewed as a general policy agenda, in which the outcomes are largely driven by the powers of national governments and other stakeholders (Huisman \& Wende, 2004). However, Witte (2006) observed that the degree of domestic changes varies remarkably. The Greek National Reports provided for the Bologna ministers' meetings in 2003 and 2005 declared that the framework for operating a National System for Quality Assurance in higher education was under consultation before the Greek Parliament. This framework was submitted to the Greek Parliament for discussion, following the Bergen meeting on May 30, 2005. Then, the law passed on July 10, 2005 and was published on August 2, 2005 (Law 3374/2005, Greek Government paper issues, FEK- 189/2005).

The role of the media in the problem formulation stage can be considerable, as was strongly maintained by Elsbach and Sutton (1992) who stated that the media act as an institutional stakeholder creating both coercive and normative pressures on organizations to conform to public expectations. Cohen (1963, p. 13) suggested that the relation worked in the opposite direction, namely media did not tell the public what to think, but were "stunningly successful in telling people what to think about". DeFleur and Ball-Rokeach (1989, p. 264) also suggested that the "agenda of the press did become the agenda of the public". Nelson (2004, p. 5) observed that "studies applying the agenda setting concept found the newspaper to be a very effective media form in setting the public's agenda". Last but not least McCombs et al. (2000, p. 7) observed that "which aspects of an issue are covered in the news-and the relative emphasis on these various aspects of an issue-makes a considerable difference in how people view that issue".

Against this backdrop of influences from the media, the objectives of the first empirical study in this book, is to answer the question "How much pressure from media was there during the 2005-2006 period regarding the adoption of quality management issues in Greece at the macro level?" In order to answer the research question of this empirical study, I followed the conceptual framework (figure 3-2) adding for this particular study the concept of agenda-setting (Kingdon, 1995). 
Researching the media's view via newspapers by employing qualitative and quantitative content analysis seems useful for understanding isomorphism in relation to the adoption of quality management at the macro level in Greek higher education. Newspapers constructed a quality management "image" for their reading public in light of pending radical changes in Greek higher education; the present study offers "cultural knowledge" of the problem situation. Furthermore, that knowledge is a key requirement in understanding and interpreting data from mixed methods research.

\subsection{Theoretical Considerations and Media Coverage}

\subsubsection{Agenda-setting}

Kingdon (1995, p. 3) defined an agenda as "the list of subjects or problems to which governmental officials, and people outside of government closely associated with those officials, are paying some serious attention at any given time". He mentioned "participants without formal government positions include interest groups, researchers, academics, consultants, media, parties and other elections-related actors, and the mass public" (p. 45). He also mentioned that interest groups may affect the public agenda "more by blocking potential items than by promoting them". Kingdon (1995) provided a framework of agendasetting and policy formulation. In his view, the agenda-setting component is best conceptualized as unrelated "streams" of problems, policies, and politics, solutions, participants, and opportunities for policy choice. Next, policy ideas are recombined and developed over the years in "policy communities" of specialists and experts. For Kingdon (1995, p. 143), "policy entrepreneurs" (people who are willing to invest resources of various kinds in hopes of a future return in the form of policies they favor) provide the linkage between ideas and decision makers. Finally, he discussed that there are structures of opportunity for ideas to become part of the agenda. The notion of "windows of opportunities" implies that during brief periods of time opportunities open up for a specific policy to become adopted. In Kingdons' model, policy windows open and close as a result of changes in the problem and political streams. He stated "policy windows open infrequently, and do not stay open long". He also noted that "sometimes the wind ow is opened by a problem that presses in on government, or at least comes to be regarded as pressing" (p. 174). 


\subsubsection{Media in Agenda-setting}

Popular media play a huge role in informing the public agenda. "In the information age, the media has become the central cultural force in society. Journalists serve a gate-keeping function on the amount of information that reaches the public" (Baran, 2002, in Kendall, 2005, p. 12). According to Dimmick (1974), editors determined the news content for newspapers through their role as gatekeepers, i.e. those who decide what will be in media (Straubhaar \& LaRose, 2002).

In 1922, Walter Lippman suggested that various media were responsible for the "picture in US heads". He argued that the mass media are the link between world events and the pictures of these events in our minds. The theory of agendasetting, according to Larson, proposed that "the public agenda—or what kinds of things people discuss, think, and worry about-is powerfully shaped and directed by what news media choose to publicize" (Larson, 1986, p. 87). Several people agreed with Lippman including Cohen's (1963) oft-quoted statement that the media may not always be successful in telling people "what to think", but in telling them "what to think about". For example, if the national media such as $\mathrm{CNN}$ and the New York Times report on 1) drought, 2) hate crime, 3) breast cancer, and 4) a car crash, in that order, the agenda-setting theory proposes that the public will rate those items the same way in terms of importance (Kendall, 2005, p. 11). Therefore, the media may not be successful in dictating how people think about global warming, but it has the capacity to make people think about drought. Lang and Lang (1966) said, "the mass media force attention to certain issues...they are constantly presenting objects, suggesting what individuals in the mass should think about, know about, have feelings about" (Kendall, 2005, p. 15).

\subsubsection{Greece and Agenda-setting around the 1999 Greek European Elections}

Harris, Kolovos, and Lock (2001) made the first published study on agendasetting in Greece. By using content analysis of six Greek newspapers during the campaign period (40 days) in the context of the 1999 European elections in Greece, they were able to compare the agendas of the press, the different parties, and the public. Their results show that the press agenda, in terms of the relative priority assigned to issues, whether in editorial, other comments, or core news coverage, strikingly differs from both party agendas and the public agenda.

Harris et al. (2001) observed that political parties, even if they manage to initiate coverage of a specific issue, do not subsequently manage to adapt the overall press agenda to their specific priorities. The authors wrote: "we do not believe 
necessarily that the press deliberately sets out to construct an agenda to influence elections, though papers may have strong party allegiances" (p. 1131). They pointed out that the overall results of their study suggested that the public and party agendas are distinctly different. They also said that "the difference between the public and the press agendas is not entirely consistent with studies in other national settings, but it may be evidence to suggest that the public does not necessarily respond to the press agenda and, that if they do converge, the movement may be from either side" (Harris et al., 2001, p. 1132). They underscored that the most important conclusion from their research was that the political parties and the press had distinct agendas, and correspondingly, the press and political parties also did not have a common view of the relative importance of issues in Greek society. What does this mean for higher education? Possible agenda-settings are discussed at the end of this part. G. Papandreou noted that in Greece "we have the media, which create panic by always looking for scandal" (1998, p. 146).

\subsubsection{Stakeholders and Neo-Institutional Pressures}

Chen and Meindl (1991) observed that media achieve their impact through consideration of interaction with the audience. They discussed that news-making is a process entailing news selection, editing, writing, information gathering, and information checking. Besides, the authors noted with Tuchman (1978), that news professionalism has developed in conjunction with modern news organizations, and journalists' professional practices serve organizational needs. Consequently, Chain and Meindl believed that the dichotomy between organizational commitment and professional allegiance that characterised traditional professionals is very much obsolete in this domain (DiMaggio \& Powell, 1983). For the purpose of their study, where they examined the construction of leadership images in the popular press, Chen and Meindl (1991) adopted a cultural perspective, examining how the news industry as a whole influences and shapes news consumers' cultural conceptions and beliefs. Hence, Chenn and Meindl treated news organizations in aggregate as an organizational field that constitutes "a recognized area of institutional life" by sharing a rather homogeneous structure and values (DiMaggio \& Powell, 1983).

In addition, Greening and Gray (1994), similar to Elsbach and Sutton (1992), stated that the media can influence an organization's reputation and in doing so exert pressure on organizations to conform to public influence. Dawkins (2005) argued that stakeholder groups can use the agenda-setting function of media by trying to gain media coverage for their activities. He quoted Frooman (1999), who stated that if the media cover their activities, stakeholders are able to inform 
potential allies about an organization's behavior, why that behavior is undesirable, and what the ally should do to change the organization's behavior.

Consistent with these views, I agree here with Chen and Meindl (1991), who contend that organizational credibility is the primary concern across news organizations, which makes both performance information and attribution history essential in the process of image construction. Nelson (2004) similarly argued that the relation between media coverage and stakeholder's perception is crucial, and therefore, organizations are concerned with their image in the media.

In contrast, Gunter (1992, p. 147) noted that the public is frequently skeptical about information from media sources like newspapers and television (Clark, Martire, \& Bartolomeo, 1984; Harris, 1983; McCombs \& Washington, 1983). Research has not explained much about the origins of either trusting or skeptical public attitudes toward the press. Gunter argued that trust in press coverage of issues or groups is more likely to be a consequence of a person's involvement with those issues and groups than of traditional predictors.

Considering the above theories and the fact that "the difference between the public and the press agendas [in Greece] is not entirely consistent with studies in other national settings, but it may be evidence to suggest that the public does not necessarily respond to the press agenda" (Harris et al., 2001, p. 1132). The following sections provide the methodology and the findings of investigating media coverage of quality assurance in Greece and how a "quality assurance image" was constructed.

\subsection{Methodology: Content Analysis of Greek Newspapers}

"In media studies, content analysis involves measuring the amount of time or space the media devotes to a person or event. Ideally, a content analysis should include all media, including television, radio, newspaper, magazines, internet and advertising. Unfortunately, this would take too much time. Most studies are confined to one or two media usually television or newspapers" (Williams \& Semlak, 1978, in Kendall, 2005). In this study too, only part of the overall media is used, namely Greek national newspapers. Figure 6-1 shows that in total, 43\% held a positive view of their newspapers although only $7 \%$ of newspaper readers were very satisfied with this type of media. 


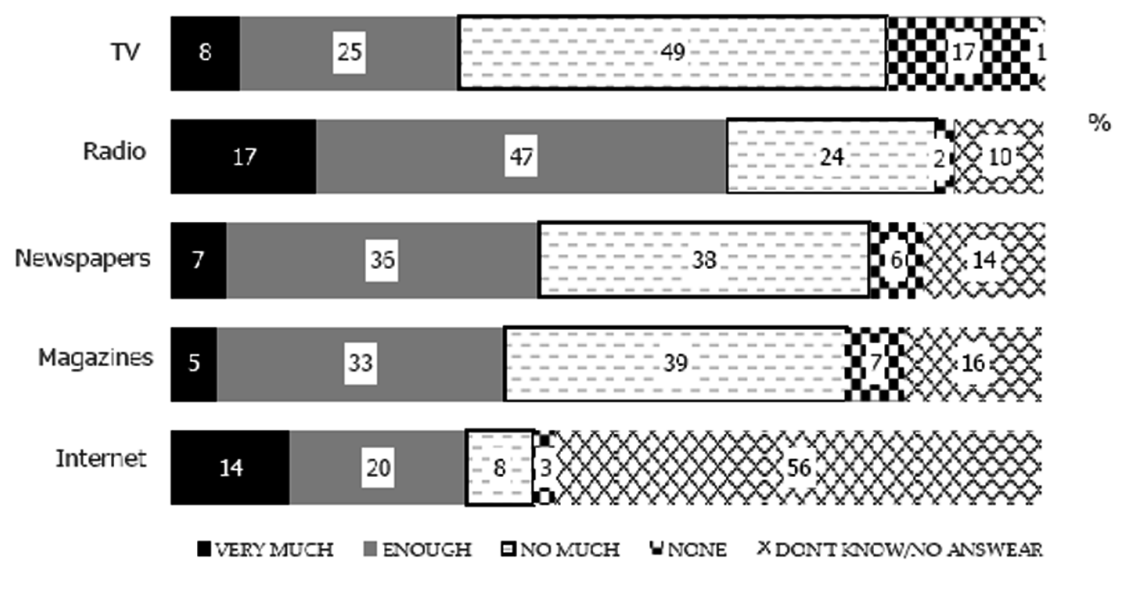

Base : MALE- FEMALE 13-70 yeays old in total in Greece

Figure 6-1 2007 media users' satisfaction

Source: translated from FOCUS

Only the radio has a higher trustworthiness in the public's eyes (or ears), but archives of radio items are not available, so that content analysis of newspaper inputs, therefore, seems the best available source for insight into media efforts to influence the public agenda. I assume that similar figures would have been found during 2005-2006, the period on which I focus.

\subsubsection{Operational Definitions and Categories}

In this study, my focus was to analyse whether articles in newspapers were for or against introducing quality assurance and which reasons were given for the point of view, interpreted in terms of coercive, mimetic, or normative pressure. The coding categories were based on the neo-institutional perspective's classification and the article's tone.

Coding Scheme for the Isomorphic Pressures Classification of the Article

The coding categories based on neo-institutional literature classification involved three indicators of pressure. These indicators, operationalized as explained in chapter 4, were coercive, mimetic, and normative pressure. Combinations of categories were also distinguished in this study: Normative and Coercive $(\mathrm{N}+\mathrm{C})$, Normative and Mimetic $(\mathrm{N}+\mathrm{M})$, Coercive and Mimetic $(\mathrm{C}+\mathrm{M})$, and Normative and Coercive and Mimetic $(\mathrm{N}+\mathrm{M}+\mathrm{C})$. Inputs mentioning quality assurance 
without any sign of isomorphic pressures were defined as category Zero (0). The object of the input was originally divided into two categories: quality assurance in general and the quality assurance law (policy) as discussed in the Greek Parliament.

\section{Coding Scheme for the Tone of the Article}

Inputs were judged to express one of three types of tone: favourable (positive), neutral, or unfavourable (negative). Nelson (2004) used Steger's (1999) content analysis of news coverage in the New York Times and Chicago Tribune served as a model for this variable. According to Nelson, favorable content implies that the person(s), place(s), or thing(s) covered in the stories are advantageous to the institutional image. In my study, the interest lies on the quality assurance image, and a similar approach was used. For example, favorable content (positive) could be from professors who perceived quality assurance as a beneficial tool, or from students' positive perceptions about quality assurance practices. Unfavorable (negative) content means the person, place, or things involved in the story/article were disadvantageous to the institution's image. In this study, an example of unfavorable tone would be threatening with as strikes, locked universities, and demonstrations against the quality assurance system or the quality assurance law. Neutral content means the primary person, place, or things involved in the story are neither favorable nor unfavorable to the image of the institution. A neutral example would be an article that presents quality assurance as a fact, without negative or positive comments. Cases where positive and negative comments were impossible to determine were also coded as neutral.

\section{Intercoder Reliability}

The researcher of this study was the primary coder. Having established the codebook and operational guidelines, reliability was informally tested in $15 \%$ of the inputs (relevant to the quality assurance issues) selected randomly by a second researcher, who independently coded a selection of inputs. This person was trained by the writer of this study in using definitions of the variables and the coding procedures. There was a large degree of agreement in the tone and in categories coercive, normative, mimetic, and Zero from the beginning. Total agreement was achieved after further discussion of the meaning of the terms. It was deemed unnecessary to change the categories or instructions substantially, as the initial level of agreement was high enough (i.e., discussions were only about 5 out of 40 articles, mostly within combined categories such as normative and mimetic, normative and coercive, and coercive and mimetic). 


\subsection{Findings}

\subsubsection{Newspapers Sampled}

As an alternative source for collecting data by hand from the newspapers, several websites in Greece provided newspaper clippings covering higher education events for their "interest group's" needs. However, they also raised the possibility of bias in data collection. The University of Macedonia, Thessaloniki (UOM), offered press monitoring information to its staff and on its university's website. Press monitoring was outsourced to a company, which selected articles on the key words: "University of Macedonia", "Education", and "Higher Education" (personal communication, February 2008). This archive best covered my research needs, because these data were perceived as the most neutral source for the purpose of this study. Data collection for analysis accordingly was derived from the UOM archives. Two other data sources were also considered, POSDEP and Alfavita ${ }^{18}$. However, they proved less complete than the University of Macedonia database and they appeared to be overwhelmingly biased against quality management.

Table 6-1 2005 monthly newspaper clippings on higher education issues in three databases

\begin{tabular}{lccc}
\hline Months & UOM & POSDEP & ALFAVITA \\
\hline January & 703 & 23 & 0 \\
February & 608 & 35 & 0 \\
March & 800 & 41 & 10 \\
April & 684 & 17 & 12 \\
May & 751 & 43 & 30 \\
June & 546 & 48 & 9 \\
July & 398 & 15 & 3 \\
August & 360 & 6 & 4 \\
September & 602 & 30 & 12 \\
October & 543 & 42 & 11 \\
November & 604 & 11 & 13 \\
December & 580 & 39 & 14 \\
\hline Total & 7179 & $\mathbf{3 5 0}$ & $\mathbf{1 1 8}$ \\
\hline
\end{tabular}

For these reasons, they were not useful for qualitative and quantitative analysis. For illustrative purposes only, Table 6-1 presents the monthly distribution of articles focusing on higher education issues for 2005. It shows the UOM data next to POSDEP and Alfavita data in order to highlight the amount of headings during each month in 2005. Clearly, the UOM database was much more complete than

\footnotetext{
18 http://www.ntua.gr/posdep/index_en.htm, http://www.alfavita.gr/typos/index.php. They are hosted by Hellenic Federation of University Teachers' Associations (POSDEP) and Educational Information Network (Alfavita).
} 
the other two.

Among the UOM data, March and May 2005 have the largest amount of newspaper inputs. These data covered issues related to higher education in general. I selected the month of May, as mentioned previously, as the month of major events related to quality assurance, such as the Bergen meeting and the quality assurance law (policy) discussion in Greek Parliament. These sources demonstrated the popularity in this "hot" month for this topic. Incidentally, the first survey of my own study was administered in May 2005.

The time frame for the present study was May 2005. The unit of analysis was the newspaper's individual inputs as a whole: articles, opinion pieces, letters to the editor, and generally any entry related to quality assurance issues. The study was based on content analysis of 751 newspaper inputs from all Greek newspapers, as available in the University of Macedonia archive. From 751 newspaper inputs from the UOM database in May 2005, a total of 255 clippings covered issues of quality assurance which were selected for further quantitative and qualitative (QUAN+QUAL) analysis.

\subsubsection{Data Analysis}

Mixed data analysis was used to examine the articles. Quantitative and qualitative data analytical procedures were utilized sequentially, beginning with quantitative analysis, followed by additional qualitative analysis, and finally quantitative analysis again. The final quantitative analysis was only possible after coding themes had been determined in the qualitative data analyses. This approach stemmed from similar analyses conducted by Wang et al. (2007). Those authors followed five of the seven stages of Ownwuegbuzie and Teddlie's (2003) mixed data analysis process (data reduction, data display, data transformation, data correlation and data integration), in order to implement a sequential mixed method analysis (Ownwuegbuzie \& Teddlie, 2003; Tashakkori \& Teddlie, 1998). Similar approaches were used in this study.

\subsubsection{Quantitative and Qualitative Analysis}

The 751 inputs originated from 54 Greek newspapers. These individual inputs were related to quality assurance and quality assurance law (policy) issues. In this first analysis, I focused on the words related to quality assurance such as "evaluation", "quality", "improvement", "Bergen", "quality assurance law" in the inputs' titles. As a further justification for choosing the month of May, I examined the use of the word "evaluation" (the nearest in meaning and most- 
used Greek term in this area) in titles during the whole year of 2005. Table 6-2 confirms how coverage varied during the period January-December 2005.

Table 6-2 Number of titles with "evaluation" in the UOM database in 2005

\begin{tabular}{lc}
\hline Months & Evaluation term \\
\hline January & 16 \\
February & 8 \\
March & 41 \\
April & 5 \\
May & 70 \\
June & 41 \\
July & 19 \\
August & 1 \\
September & 2 \\
October & 2 \\
November & 5 \\
December & 6 \\
\hline Total & $\mathbf{2 1 6}$ \\
\hline
\end{tabular}

During 2005, the word "evaluation" appeared 216 times - 41 times in March and June, only 19 times in July, and 70 times in May alone. Figure 6-2 presents this trend and very clearly shows that May was the peak "quality assurance period".

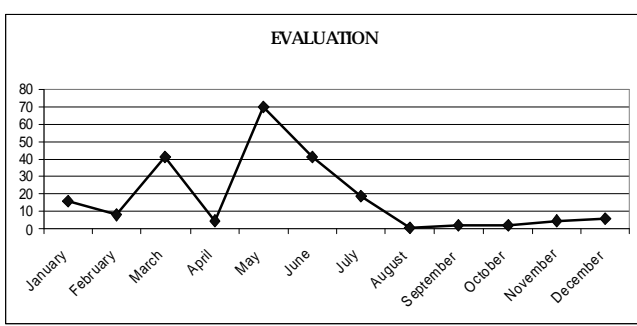

Figure 6-2 "Evaluation" trends for 2005

Prior to discussion of the coding analysis (in depth-analysis), a brief look is taken at the distribution within the month of May to see the presence of "evaluation" or "quality assurance" as terms in headings in the UOM database (Figure 6-3).

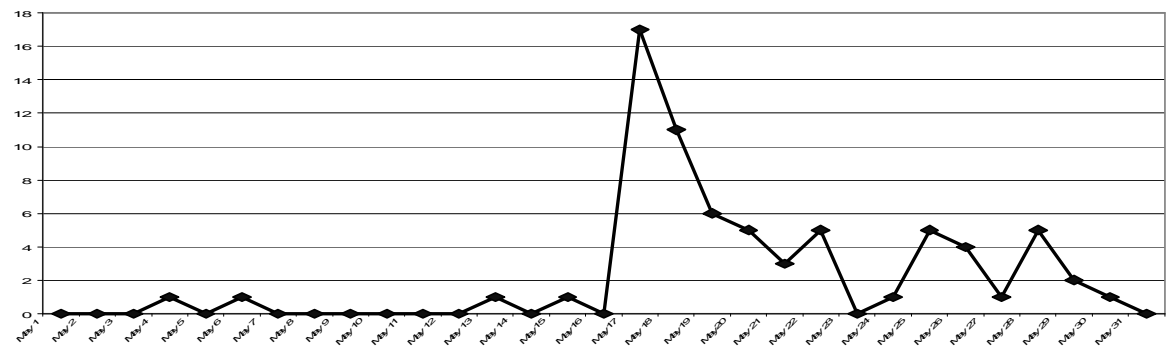

Figure 6-3 "Evaluation" trends during May 2005

May $17^{\text {th }}$ and $18^{\text {th }}$ appeared to be the most popular dates for this term. This peak period correlated with the Bergen meeting which took part on 19-22 May, 2005 and the "presentation/exposition" of the quality assurance frame law by the Minister of Education on May 17, 2005 before she traveled to Bergen. 
Table 6-3 "Evaluation" trends covered by Greek newspapers during May 2005

\begin{tabular}{cl}
\hline "Evaluation" & \multicolumn{1}{c}{ Newspapers } \\
\hline 9 times & Eletherotipia \\
7 times & Ethnos \\
5 times & To Vima, Kathimerini \\
4 times & Bradini, Ta Nea \\
3 times & Aggelioforos, Typos tis Thessalonikis \\
2 times & 10 newspapers \\
1 time & 10 newspapers \\
\hline Total 70 & Total 28 newspapers \\
\hline
\end{tabular}

Table 6-3 presents the Greek newspapers from which the inputs originated during this month. Few prominent newspapers ${ }^{19} \quad$ discussed these articles with the term "Evaluation" in their titlesEletherotipia used "evaluation" nine times, followed by Etnos seven times, Vima five, and Kathimerini five. Twenty-eight newspapers followed this trend during May 2005.

Table 6-4 Popular words in May 2005 headings

\begin{tabular}{lc}
\hline \multicolumn{1}{c}{ Words } & Times \\
\hline Evaluation & 70 \\
Quality & 4 \\
Bergen & 10 \\
Frame-law for QA & 11 \\
Improvement & 1 \\
Locked, marches, strikes & 28 \\
\hline Total & 124 \\
\hline
\end{tabular}

Looking at the frequencies and the spread of media coverage during May 2005, this month could fruitfully be divided into three periods of media interest: pre-Bergen meeting (May 116), with 33 articles; during Bergen meeting (May 17-22), with 142 articles; and post-Bergen meeting (May 23-31), with 76 articles. Extra words also used by newspaper headings and related to quality assurance were: "quality", "Bergen", "Frame-law for quality assurance", and "improvement". "Locked/closed universities", "marches", "protests" and "strikes" appeared 28 times in May 2005 and seemed popular (Table 6-4).

${ }_{19}$ Detailed information on Greek newspaper circulation could be found at Athens Daily newspaper Publishers Associations: http://www.eihea.gr. 


\subsection{Neo-Institutional Pressures and Media Coverage}

\subsubsection{Qualitative Analysis}

The following examples were derived from a qualitative analysis of the articles that developed into categories and patterns. This analysis facilitated a more indepth background understanding of the coding categories.

\section{Coercive Positive Articles}

Articles which covered political parties that were pro quality assurance were categorized as coercive positive.

\section{Coercive Negative Articles}

Terms such as "strikes", "closed", "locked universities" were associated with attempts against quality assurance practices from the point of view of students and professors. These articles were characterized as coercive negative. Articles which covered political parties that were against the quality assurance were categorized as coercive negative as well.

During the last week of May 2005, when the quality assurance frame-law went to the Greek Parliament for discussion, the media covered issues with titles such as "universities locked", "closed universities". These titles exerted negative coercive pressure. Although I read these articles numerous times, it was difficult to categorize them as coercive negative since these articles started with strikes and universities but later discussed the Greek Rectors' Council meeting (which took part during the last days of May 2005). In the middle of every article there was a sentence such as:

The rectors in their meeting said yes to evaluation or quality assurance law but universities required extra finance in order to survive.

or

The rectors are in favor of quality assurance and universities must evaluate themselves with reliable processes. The Ministry of Education must undertake its responsibilities in order to solve the well known problems in Greek universities by using the evaluation's results.

Finally, these articles were coded as coercive negative, as their title featured the word "strikes" or "marches", and one sentence in a large article was not enough to categorize it as normative positive or normative and coercive positive. 


\section{Normative Positive Articles}

An example of a normative positive article is the title "The Time of Collisions Came". Very clearly, this article stated that the Minister of Education reacted aggressively against the POSDEP. The teachers' association (POSDEP) refused to accept the evaluation process for universities. This article stated that POSDEP's decision was a "trade" or "horizontal union" decision and was antithetical to the majority of the professors' opinions regarding quality assurance. The article claimed that it was impossible for someone to be negative towards quality assurance processes, as quality assurance and education are parallel.

Another example of normative positive was directly connected with professors' perspectives and positive opinions of quality assurance law or quality assurance practices and clearly expressed their opinion by sending letters to the newspaper editor.

\section{Normative Negative Articles}

Normative negative articles were critical of the quality assurance law. They referred to the vagueness of the quality assurance criteria; criticized the number and the qualifications of the external examiners in the quality assurance process or pointed out the mistakes that appeared in the draft of the quality assurance frame-law.

\section{Mimetic Positive Articles}

Examples of mimetic positive were articles which presented US and European quality assurance processes and claimed that quality assurance is a prestigious international process.

\section{Coercive and Normative Neutral Articles}

An example of an article difficult to categorize was one that covered issues such as students who were against quality assurance and who participated in demonstrations; additionally, the same article quoted a parliament member who was in favor of quality assurance saying that quality assurance was needed in higher education and beneficial for the university. The last part of this article could be characterized as normative positive and the first part as coercive negative, besides the title of this article was "Marches in Thessaloniki against Quality Assurance Law in Higher Education" and was therefore considered coercive negative. I categorized such articles on the whole as coercive and normative neutral. 


\section{Coercive and Mimetic Positive Articles}

An example of a coercive and mimetic positive article was one which stated that quality assurance was required for universities. This process was adopted in Bergen (mimetic positive) and universities who did not participate in quality assurance processes would not receive European financing (coercive positive).

Coercive and Mimetic Negative Articles

Newspapers during May 17-19 and 30-31 ran articles mostly of the type coercive and mimetic negative. The "anti-Bergen week", as cited in these articles, was connected to strikes and marches by students and some professors who were against the Bergen meeting, the Bologna Process, and the evaluation. As can be seen from above, the quality assurance theme was well presented in newspaper coverage in May. Coercive and mimetic negative included articles which wrote that Greece adopted the quality assurance system as a Bologna Process requirement (mimetic negative). Moreover, students and professors were against it and participated in strikes and marches against this process (coercive negative).

Articles without taking a stance (Zero)

This type of category included articles which mentioned quality assurance but did not signal either positive, negative, or neutral attitudes and moreover, did not exert any clear pressure against or for quality assurance.

\subsubsection{Quantitative Analysis}

This analysis provided information on isomorphic pressures and inputs tone. A total of 255 clippings from all newspapers in May 2005 were analyzed for this phase. Each article was coded once. Table 6-5 presents the overall findings of this study.

Table 6-5 Quantitative overall findings

\begin{tabular}{|c|c|c|c|c|c|c|c|c|c|}
\hline \multirow{2}{*}{$\begin{array}{c}\text { Standpoint } \\
\text { Pressure }\end{array}$} & \multicolumn{2}{|c|}{ Positive } & \multicolumn{2}{|c|}{ Neutral } & \multicolumn{2}{|c|}{ Negative } & \multicolumn{2}{|c|}{ Sub-Total } & \multirow[t]{2}{*}{ Tota } \\
\hline & $\mathrm{QA}$ & $\mathrm{QA}$ & $\mathrm{QA}$ & $\mathrm{QA}$ & $\mathrm{QA}$ & $\mathrm{QA}$ & $\mathrm{QA}$ & $\mathrm{QA}$ & \\
\hline & & Law & & Law & & Law & & Law & \\
\hline Coercive & & 33 & & 5 & & 48 & & 86 & 86 \\
\hline Normative & 2 & 37 & & 3 & 1 & 29 & 3 & 69 & 72 \\
\hline Mimetic & 3 & 16 & 1 & 9 & 2 & 9 & 6 & 34 & 40 \\
\hline $\mathrm{N}+\mathrm{C}$ & & 3 & & 0 & & 7 & & 10 & 10 \\
\hline $\mathrm{N}+\mathrm{M}$ & & 4 & & 2 & & 8 & & 14 & 14 \\
\hline $\mathrm{C}+\mathrm{M}$ & & 7 & & 2 & & 14 & & 23 & 23 \\
\hline$N+C+M$ & & 0 & & 1 & & 2 & & 3 & 3 \\
\hline Zero & & 0 & & 7 & & 0 & & 7 & 7 \\
\hline Total & 5 & 100 & 1 & 29 & 3 & 117 & 9 & 246 & 255 \\
\hline
\end{tabular}


Quality assurance (QA) and quality assurance law (QA Law) will be treated together from this point on, as quality assurance as a general term appeared only nine times. The newspapers' overall coverage of quality assurance was somewhat more unfavorable/negative (120 times) than favorable/positive (105 times), with 30 items being neutral $(11 \%)$. Thus, we can see that the tone was a little more $(47 \%)$ negative than positive $(41 \%)$. In this analysis, the tone is derived from the body of the input and not only from their title or their pictures. Total frequencies for isomorphic pressures and tone code are indicated in Figure 6-4.

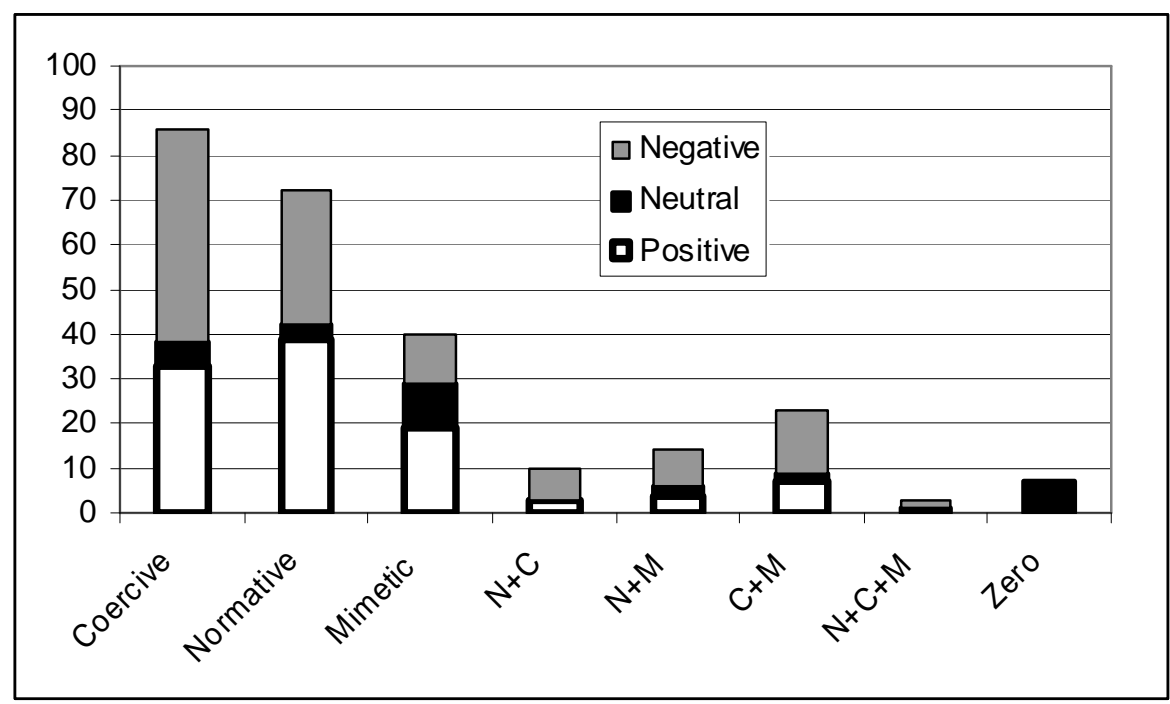

Figure 6-4 Total frequencies of isomorphic pressures and their tone

A coercive negative tone appeared in 48 articles, much more than a coercive positive tone (33). Normative pressure with positive tone appeared in 39 articles, more than normative negative tone (29). Mimetic positive tone appeared in 19 articles, negative in 11 articles, and neutral in 10 articles. The category coercive and mimetic $(\mathrm{C}+\mathrm{M})$ appeared 14 times negative, 7 positive, and 2 neutral. The category normative and coercive $(\mathrm{N}+\mathrm{C})$ appeared 7 times negative, 3 positive, and none neutral. Additionally, the category normative and coercive and mimetic $(\mathrm{N}+\mathrm{C}+\mathrm{M})$ is associated with negative tone and quality assurance law 2 times, with neutral tone once and positive tone zero times. The Zero category articles were by definition neutral (7 times).

As noted earlier, May 2005 was divided into three periods of media interest. In the pre-Bergen meeting (May 1-16) out of 33 articles, 15 were positive, 15 
negative, and 3 neutral. Around the Bergen meeting week of articles (May 17-22), out of 146 articles, 70 were negative, 55 positive, and 21 neutral. Finally, in the post-Bergen period (May 23-31), the media covered 76 articles of which 35 were negative, 35 positive, and 6 neutral (Table 6-6). Additionally, the popular terms "locked", "closed", "marches", and "strikes", featured in 52 titles, most of them (28) in the post-Bergen period.

Table 6-6 Articles' tone during May 2005

\begin{tabular}{lccc|c|c}
\hline Periods & Positive & Neutral & Negative & Total & $\begin{array}{c}\text { "locked, } \\
\text { marches } \\
\text { \& strikes" }\end{array}$ \\
\hline Pre Bergen & 15 & 3 & 15 & 33 & 7 \\
Around Bergen & 55 & 21 & 70 & 146 & 17 \\
Post-Bergen & 35 & 6 & 35 & 76 & 28 \\
\hline Total & $\mathbf{1 0 5}$ & $\mathbf{3 0}$ & $\mathbf{1 2 0}$ & $\mathbf{2 5 5}$ & $\mathbf{5 2}$ \\
\hline
\end{tabular}

Views (negative vs. positive) or in other words the image of quality assurance were balanced in the pre- and post-Bergen period. Only during the Bergen meeting, which was directly connected with the presentation of the quality assurance frame-law in Parliament, was there a predominantly negative tone in newspaper coverage.

Previously, I counted words from the University of Macedonia's database of newspaper clippings. In this second part, the analysis consisted of manual content analysis for all 255 articles, which later was "quantitised" for statistical purposes. For this second, qualitative analysis, I downloaded the full documents from the University of Macedonia archive. I detected a few times that the database had interchanged the first and second lines of a title; therefore the entire title had to be taken into consideration in counting hits for "locked" as well as other terms. I also found that single items in the database marked "Education" in fact included several articles on the same page of the newspaper. Therefore the difference in numbers could be explained by the two different approaches for analysis (52 original clipping vs. 28 UOM archive).

Rectors and students who were in favor of quality assurance as well as some professors and students who were against quality assurance voiced their opinions in articles entitled "Locked in the Universities". Similar articles, as noted previously, appeared 52 times in May. Table 6-7 provides the "locked" analysis in newspapers articles. Unfortunately, May 30 was when my first survey (related in 
Chapter 8) was administered, and that timing might be connected with the low response rate.

Table 6-7 "Locked" and similar terms in newspapers titles

\begin{tabular}{lccccccccc}
\hline \multicolumn{1}{c}{ Isomorphic pressures } \\
\hline Tone & Normative & Coercive & Mimetic & Zero & N+C+M & N+C & C+M & N+M & Total \\
Negative & 0 & 29 & 1 & 0 & 1 & 4 & 5 & 1 & 41 \\
Positive & 9 & 2 & 0 & 0 & 0 & 0 & 0 & 0 & 11 \\
\hline Total & 9 & 31 & 1 & 0 & 1 & 4 & 5 & 1 & 52 \\
\hline
\end{tabular}

In this light, it was understandable that one rector in a letter explained that he did not want to participate in my research (chapter 8), as the quality assurance law was under discussion. Such coincidences contributed to my choosing mixed methods analysis, to triangulate findings among several empirical studies within this research.

\subsection{Summary and Conclusion}

The purpose of this chapter was to address how media covered quality management in terms of quality assurance policy during 2005. The analysis of quality assurance relied on the agenda-setting concept in combination with the idea of isomorphism. I observed how the image of quality assurance was constructed through media coverage during a peak period in Greece. The study investigated the amount, isomorphic character and tone of this coverage. The findings were part of an overall trend of higher education coverage. Newspapers in Greece during 2005, published more than 7,000 articles related to higher education field (first level agenda-setting), which shows that higher education issues were of interest to the professional editors and editorial staff who provided Greek society the priority of what to think about. One could observe that the media blitz about strikes, locked universities and marches forced the issue into Greek thinking because media were telling average Greeks what to think about. The more coverage an issue receives, the more concern individuals have with the issues (McCombs et al., 2000). As discussed earlier, the second-level of agenda setting examined how media organizations select and present certain characteristics and properties of an object and its attribute. The findings from 255 inputs from May 2005 confirmed that quality assurance was well represented in the newspapers.

One might claim that this conclusion is limited by the range of my sample. Scholars (Miles \& Huberman, 1994; Patton, 2002; Teddlie \& Tashakkori, 2009) 
stressed that "sampling of a politically important case" as used in this study, "is a special or unique case that involves selecting politically significant or sensitive cases for study". In this study, I examined in detail only inputs from a one-month period. May 2005 was selected, because media coverage was associated with the major quality management event in Greek higher education, the publication of the first national quality assurance law.

Through manual content analysis, it was shown that newspapers represented mostly coercive statements on the quality assurance law. Overall, over one-third (35\%) of the newspapers voiced coercive pressure. Besides, 69 (28\% of total) dealt with normative pressure, $20 \%$ dealt with a combination of categories, and less than $14 \%$ (34 articles) dealt with mimetic pressure only. At the center of the debate over media coverage, the issues are about tone: was the press coverage more likely to have a negative emphasis? Overall, $47 \%$ of all inputs in the study displayed a negative tone toward the image of quality assurance, while $41 \%$ were mainly positive, and $11 \%$ neutral. More than $55 \%$ of coercive pressure inputs had a negative tone (48 out of 86 ). Entries with combinations of isomorphic categories also in majority had a negative tone $(67 \%)$. In contrast, a positive tone was found in 39 out of 72 inputs characterized as giving normative pressure (54\%). Evidence of a positive tone $(47 \%)$ was also found in mimetic pressure inputs.

Looking at the timing of articles, the findings of this examination showed an equilibrium between negative and positive tones before and after the middle of the month: the negative majority, as noted in the previous paragraph, was the result of one week's news coverage during the week when the quality assessment law was submitted to parliament and when the Bergen meeting took place. Examining the Greek newspapers during May 2005, mostly negative coercive headings were the main vehicle to construct the image of quality assurance in Greek society. Titles such as "Universities before the Quality Assurance" (as in, "before the court"), "Marieta ${ }^{20}$ step down", and "You [the Minister] go to Bergen, we [the students] are going to the street" did not create a favorable image for quality assurance. It is interesting also to see that negative titles such as "locked [universities]", "marches", or "strikes" appeared in 52 articles, obviously such headings were in negative coercive articles (41 out of 52). However, I found that nine normative positive and two mimetic positive articles had negative titles with terms such as "locked". Articles of positive tone with negative headings showed that the image of quality assurance was confused during this period, or maybe newspapers sought "fancy" headings for their articles. The headings in the majority of newspapers were more critical to quality assurance than the actual

\footnotetext{
${ }^{20}$ Ms Marietta Yiannakou it was the Minister of Education
} 
text that readers might find further down in the articles. That means that perseverance of readers was needed to read the whole article in order to get the correct image. Readers who only skimmed headings would get a much more negative image about quality assurance. The largest group of items among my findings related to coercive inputs (86 out of 255) and from those more than $55 \%$ (48) were negative. In addition, from the pro-Bergen to the post-Bergen period inputs sporting headings with "strikes" and "closed [universities]" grew by over three times (28). This finding could be explained by the growing political trend against quality assurance.

The study attempted to ascertain the origins of the images explored during the month of evaluation (May 2005). It analyzed which perceptions were transmitted via mass media. The object was not so much to question the perceptions of evaluation in Greek higher education, but rather to identify the circumstances under which the quality assurance policy emerged. Quality management as noted earlier (chapter 2), suggests a transformation process requiring a fundamental shift in management practice and culture. Quality assurance is often seen in higher education studies as a policy instrument of governments, and as a management tool within higher education institutions. In Greece, like in many other European countries (Schwartz \& Westerheijden, 2004) quality assurance started as a governmental policy. Huisman and van Vught (2009, p. 21) noted that "the Bologna process became a major higher education policy context at a European-wide scale". The general consensus was that Bologna was the main reason that drove the Greek Government to develop the quality assurance policy for its higher education institutions. Looking at the policy process that emerged from Bologna as it pertained to the Greek government, it created a "window of opportunities" during 2005, and the Minister of Education took advantage of "the window", acting as a "policy entrepreneur". Ravinet (2008, p. 365) pointed out that follow-up activities and the stocktaking exercise in the Bologna Process would "allow comparisons, and create effects on socialization, imitation, and shame-which can be powerful means of coercion". From that perspective, an "open window" for the adoption of a quality assurance policy in Greece was created in 2005 by the occurrence of the Bergen follow-up meeting. This window had opened previously during 2001, and 2003; however, then there had not been policy entrepreneurs taking advantage of it.

In this chapter, quality management was associated with the national quality assurance policy. The significant amount of attention given to the quality management law (246 out of 255 newspaper articles) in conjunction with the predominance of coercive arguments combined in such predominance so that consideration of quality management was placed under the magnifying glass of 
legitimacy. In such a case, the adoption of quality management in higher education institutions may become simply "myth and ceremony". Tolbert and Zucker (1983, p. 27) also claimed that "legal requirements do not always ensure adoption". In other words, the adoption of the law might happen, but would that lead to actual adoption of quality management practices inside universities?

Following DiMaggio and Powell's approach, Pollitt (2003, p. 129) further expressed his thoughts about why mimetic isomorphism occurs; "when under conditions of uncertainty about what the best thing to do may be, an organization concludes that the safest course is to copy what has been done by some apparently successful or high status organization in their field". He continued, "this should confer legitimacy, even if performance does not improve-one always has the defense that one was imitating the best". However, Meyer and Rowan (1977, p. 359) noted that "organizations must not only conform to myths but must also maintain the appearance that the myths actually work". In such cases, it is possible that the adoption of institutionalized practices (or "myths") may bring legitimacy, but it can also be ineffective or unrealistic for the organization (in Greek case, for Greek universities). Scott (1991) mentioned that in such cases, the organization may submit ceremonially to rituals and symbolic elements of the managerial technique or innovation without taking it seriously. Such "ceremonial behavior" can be linked to the concept of loose coupling, as used by Weick (1976, p. 5), which occurs when "an organization's structure is not coterminous with its activity".

This study, and aspects from the environmental scan of Greek higher education (chapter 5), provided "cultural knowledge" about the adoption of a quality assurance policy in May 2005. The study's results suggest that Greece may be a difficult place to study adoption of quality management by interviewing and surveying individuals. Accordingly, it motivated me to first try to provide a picture of adoption of quality management through a document analysis in my next study. The media may have set the parameters in which citizens discuss public events, but a question of more direct pertinence for the adoption of quality management in higher education institutions is what influenced the behavior of universities themselves. The next empirical studies therefore further consider how academia, at the meso and micro levels, dealt with isomorphic pressures around the adoption of quality management. 


\section{Which Universities Voluntarily Invite an External Evaluation?}

\subsection{Introduction}

The aim of this inquiry was to understand the relationships between neoinstitutional pressures and university characteristics that influence the adoption (or lack of adoption) of quality management at the meso level in Greek higher education. In the absence of any national quality performance monitoring system (until 2006), eight of the twenty-one Greek public universities voluntarily participated in the EUA Institutional Evaluation Program (EUA-IEP). Furthermore, until recently, almost everyone in Greece who talked about "evaluation", referred to these EUA-IEP exercises (Kyriazis, 2006; YPEPTH, 2005, 2007).

The central actors in the EUA-IEP's evaluation teams are university rectors and presidents who have both knowledge of and experience with different European higher education systems (the peer review). They always come from other countries than where the evaluated university is located. As noted earlier, the aim of EUA-IEP is to offer universities an external evaluation that takes account of their external and internal environment. It evaluates current conceptions of strategies and activities and promotes internal quality in universities. The EUA evaluators' reports are the main output of their reviews. These reports were made after two peer review visits to each of the universities and included the evaluators' judgments and recommendations. Reports are structured in principle according to EUA's guidelines, in order to provide similar types of recommendations to individual universities. These reports represent, therefore, key events in the quality management of these eight Greek universities and of the higher education sector as a whole. Fetterman (1998) stated that documented key events are valuable resources and he claimed that key events provide a lens through which to view a culture.

\subsubsection{Methodology}

The mixed method approach was, in my estimate, the most appropriate methodology for this study. The previous chapter's content analysis of the media offered a picture of the "quality management image". Now, I shall proceed with 
a qualitative (QUAL) exploration, which employs content analysis of eight EUAIEP evaluators' reports.

\section{Operational Definitions and Categories}

The reports provide an evaluation of a university. IEP's focus, as defined in the EUA-IEP guidelines, is on a university's mission statement, its strategic planning, facilities and resources, learning and teaching, quality assurance, and organizational structures. EUA-IEP visiting teams made their examination based on the basic four questions (section 2.2.1). The EUA visiting team's role is clear; the review process "is consultative and supportive and aims to develop quality as a central value guiding the university's strategic development and management". In almost every report, the reviewers underscored that:

The EUA or former CRE Institutional Evaluation program does not assess the quality of the university but the available mechanisms for quality assurance... The review team's mission is to scrutinize the university's organization and its mechanism for quality assurance; therefore, [the review team] wish to highlight current strengths and weaknesses and to voice some recommendations that [are meant to aid] in future development of the university. ${ }^{21}$

I began this research with the expectation that reports would explore the three categories of themes highlighted in the conceptual framework of this study, namely isomorphic pressures, university characteristics, and quality management. The categorization scheme for the content analysis followed the variables' operationalizations as set out in chapter 4 of this study. As I have already indicated, the university mission and decision-making structures are equal in all 21 universities.

\subsection{Findings}

\subsubsection{Sample}

The data of this study were provided by the EUA and consisted of eight evaluators' reports. Some universities that have participated in the EUA-IEP provided the report on their websites and noted that they are "EUA members". Other universities circulated this report to their members by e-mail or distributed it through the Public Relations Office of the university. Another published its

\footnotetext{
${ }^{21}$ Quotes without references to protect anonymity of the universities
} 
report on-line on the university's website stating that it "formally certifies the completion of an ambitious endeavour".

\subsubsection{Data Analysis}

The data were analyzed by hand using qualitative content analysis procedures. Each report was analyzed against the three categories of themes. Universities will be portrayed in general terms; they will remain anonymous so as to protect their integrity and privacy.

\section{Reports' Characteristics}

The majority of the eight reports were organized in the same manner. The total length of the eight reports varied from 17 to 25 pages. The reports reflected similar issues and concerns. Each report concluded with recommendations to the university. The reports were published in the period from 1999 to 2005 . Each of the eight reports was written by different EUA teams.

\subsubsection{Isomorphism Pressures through the Eyes of EUA-IEP Evaluators}

This section examines reports on neo-institutional pressures. Each report was analyzed against these three pressures: coercive, normative, and mimetic.

\section{Coercive Pressure}

None of the eight reports appropriately explained coercive pressure in sufficient detail. Throughout the reports, I was only able to find general statements such as:

Systematic quality assurance procedures are not yet developed in Greece and there are no normally formal mechanisms of quality management in the universities.

Systematic quality assessment is hardly developed in Greece; recently the government tried to impose some activities (disciplinary review) but these were successfully resisted by universities and public opinion.

A central evaluation committee established under the Law of 1992 did not receive the cooperation of the academic community. In 1995, the National Educational Council was created, which aimed to evaluate institutional quality, but five years later it has yet to function.

The majority of the reports continued in the same vein: 
A 1992 law called for quality assessment at Greek institutions of higher learning, but has failed to be implemented.

There is no external quality assessment system in Greece, although the Ministry of Education tried to introduce it but did not succeed due to enormous resistance of the universities.

The team learned that new legislation stipulating a standardized system of Quality Assessment in Greek higher education is imminent.

Coercive pressure did not seem to play a significant role in the decision to invite the EUA-IEP since this action was made voluntarily by the university rector and approved by its Senate. As mentioned above, all reports consistently wrote that there was no pressure from the state for adopting quality management practices. The only, lightly coercive pressure was that at a certain time, the Greek government subsidized universities to undergo an EUA-IEP evaluation.

Other comments by the reviewing teams dealt with the nature of the Greek university system. An example is the following statement: "The review team realizes that the Greek university system, compared to other European countries, has an excessive control of the Ministry of Education". On the other hand, "the university has very strong academic freedom".

Evaluators highlighted the state control of Greek higher education, along with the fact that the funding process uses only quantitative indicators. Beyond that, the peer reviewers mentioned that Greek universities are funded with smaller budgets than other European universities. Also, the fact that university studies in Greece are free of charge, including free books, meals, and in some cases accommodation was underscored in virtually every report. Obviously, throughout these reports, it can be concluded that these remarks are not perceived as coercive pressure influencing adoption of quality management.

\section{Normative Pressure}

All eight universities that participated in EUA's evaluation were seeking feedback about their overall performance. Personal communication with four rectors or vice-rectors indicated that they participated voluntarily in this process for performance improvement at their university. For this purpose, they perceived the comments and recommendations as a good starting point. One participating university expressed it as: "The self-evaluation report constitutes an accurate and sincere attempt to depict [University X] with special emphasis on its strengths 
and weaknesses as well as the opportunities and threats in its development". It is especially noteworthy that the university stated in positive tones that:

Its participation in the EUA evaluation process was a step of major importance. It is quite obvious that the conclusions to be derived from these procedures, regarding not only the content of the final report of the review team but also every step of the process as a whole, will have significant impact on the effective, efficient and integrated establishment of the evaluation procedures for our university.

The EUA self-assessment reports suggested normative pressure, especially by their rectorates' management commitment and by the fact that these universities participated in a professional association, namely the EUA. Kyriazis (2007) noted: "It is at least a paradox to hear reactionary voices (regarding quality assurance issues and evaluation process) in Greece when at the same time 17 Greek universities and the Rectors' Conference participated in EUA". And more than just being members, almost half of these 17 universities have voluntarily invited the EUA-IEP. Taking into consideration the member benefits and services provided by EUA, all explicitly aimed at making universities more professionally organized, autonomous actors, it is possible to believe that being a member of EUA automatically enables the constituent to garner the quality assurance initiatives. Thus, Professor Kyriazis could not understand the paradox of why universities would not wish to participate in quality assurance activities and yet be an EUA member.

Normative pressure by the EUA-IEP was usually based on the university's cooperation with external stakeholders in order to improve the university's capacity for planning educational programs, continuing education, and research. Specific cases in which a university had taken into account normative pressure by external stakeholders' perceptions included:

the university's inviting members of the community to participate in informal departmental advisory committees before introducing changes in the study programs; obtaining feedback about the [X program] training and by improving the dissemination of information within the business community; creating a data base with the [University's X's] technological expertise, and organizing an annual conference with the university and their main business partner.

In a similar vein another team wrote:

It is fully acknowledged that the rector has many good ideas already in progress, which include the Friends of [X University] Association recently established, as 
well as the formalized partnership with the local municipalities which has been recently signed bringing the local world of business together with the university in a range of projects.

These types of comments demonstrated a certain degree of development of quality management. At the same time, stakeholder relations may provide normative pressure in themselves. Absence of this type of relationship implies a lack of normative pressure from stakeholders. Visiting teams pointed that lack of connections out in three of the reports, in the other five reports they made recommendations for the universities to utilize these types of relationships. Some remarks expressed the team's suggestions in this regard:

Recommendation: Local community stakeholders should be consulted thoroughly on project research needs to benefit the region.

Observations and recommendations: while the self-assessment report noted a weak connection between the university and the local economy, the participants in the discussions found that this was due more to the country's economic structure than the university's unresponsiveness.

In this latter case, the review team did not have "occasion to meet with the university's outside partners". Accordingly, the team wrote:

The direct involvement of [University $\mathrm{X}$ ] in the activities of surrounding business and firms does not seem to be as strong, however. A lack of development-oriented industries in the region was cited as a cause.

An alternative source of normative pressure by stakeholders that might influence the adoption of quality management may be found in the alumni network. In one report, the recommendation was that "organized involvement of alumni students might provide a link between the university and the outside world, reinforce the [University $X^{\prime}$ s] regional importance, and... serve as a source for donations, in addition to allowing the university to track the careers of its students as stakeholders in society at large". Since none of the other reports covered the subject of the institution's alumni, I am unable to account for the normative pressure within the various universities with regard to alumni networking. Nevertheless, it may be assumed that as in many other European countries, alumni are not organized into a powerful network that could put pressure on a university. 


\section{Mimetic Pressure}

The EUA evaluation by earlier adopters perhaps suggested their absorption of normative pressures. For the later adopters, it is possible that this practice implies a mimetic pressure, because later adopters "learned" about the EUA's benefits and the rationale of the evaluation process from early adopters and therefore decided to participate. Communication through Greek universities is fairly complete since there are only 21 universities total and each is quite aware of what the others are doing.

Since none of the eight reports addressed issues that indicated mimetic pressure, which influence the ability to adopt quality management in Greek universities, the data from the analysis of these reports does not provide any explanation for the mimetic pressure on the various universities.

My study supported the premise that calling in the EUA-IEP as part of quality management occurred as a result of normative pressure. However, for these cases there was no evidence available that thoroughly explained the degree of the normative or possibly mimetic pressure, which might be a considerable limitation of this study.

\subsubsection{University Characteristics}

The data from the reports were used to provide characteristics of the eight universities that participated in quality management.

Age

The participant universities fell into two age categories: old and new. Six were old universities and two were new. It is apparent that the typical participant was an old university.

\section{Size}

The participants fell into two size categories: medium and large. Of the eight universities, seven were from medium-size universities and one was a large-size university. There was no small university among the EUA-IEP participants.

The size indicator may be flawed, as the reports pointed to "the perpetual students", and this designation received direct mention almost in every report. The following statement raises a concern about the "permanent" student: "Given that there is no provision in the national legislation for terminating student status, [University X], like other universities in Greece, has a large number of inactive 
students". However, as this situation applied equally to all universities involved, it did not create a bias in my indicator.

\section{Location}

The participants fell into two location categories: peripheral and urban. Of the eight universities, six were peripheral universities and two were urban.

Comparing one report to another regarding the location coverage yielded some noteworthy observations. A report stated:

One can't discuss higher education in Greece without being aware of the dominance of Athens and Thessaloniki in all respects: demographic, cultural, economic, and also concerning universities; you'll find about $70 \%$ of the students in those two places. The preferences for Athens and Thessaloniki are obvious, only a very small percentage had as a first choice to studying in one of [X university's] academic faculties (schools).

Other remarks were considerably more critical of the importance of location:

Allocation of research money is also primarily an Athens business. So it is essential for most to be present at the right meeting or in meeting the right person in Athens. Together with this centralized approach to decision-making, there is the dominance of party politics at all levels. Some even used the expression that universities are prisoners of politics. That is surely exaggerated but was noticeable in many ways. Good relations with the minister, or his top-advisers, are [of] crucial importance, but at the same time, the Greek political system has a tradition of a frequent change of ministers.

\section{Range of Studies}

The studied universities all were multi-disciplinary universities.

\section{Mission}

The major concern regarding the mission of universities was: "in Greece, most of the universities do not have Statutes or their own Internal Regulations because even the internal structures are defined by the Ministry of Education (by the frame law)". Another report wrote the following:

It does not seem to be common practice in Greek universities to specify institutional or mission statements, which could be internalized in different faculties and departments. However, it is crucial for universities to work out their own profile and set the institutional policies for [their own] development. The 
review team suggests that coordinated action across all departments and schools be initiated to debate and agree on the Mission of the University.

Similarly, another report noted: "The broadness of the mentioned mission hardly distinguishes [University X] from any other university". Still another report stated:

The review team strongly recommends to debate a new mission statement for the university, trying to formulate a new statement that reflects the new external environment and that is framed based on the special core competencies and specialization of the university. The debate on this issue is a good means to strengthen the identity of [the] university.

This later statement made by the EUA-IEP review team seems to acknowledge what was previously stated about the Greek high education in that higher education is strongly controlled by the Ministry of Education under the frame law $1268 / 1982$; as such, there is little difference between the state-run universities that would require a specific mission statement unique to each university since they operate under the same law, which controls much of their operationalization to the external environment; certainly, it requires little differentiation between them. For the EUA-IEP review team to suggest that a "new mission statement" is required for a state operated university indicates that perhaps each Greek university should include localization and situationalization as unique qualities worthy of setting apart what University $X$ offered apart from other universities: specifically core competencies that relate to the relevance of the local community's needs and notable professors' fields of expertise. Certainly, that viewpoint would only occur to someone outside the Greek HE system of thinking, since uniformity under frame law is observed by all state operated universities. This comment by the EUA-IEP review team reveals the value of an external review board so that new thinking can be applied to Greek higher education and how it can work towards reinventing itself-a completely new manoeuvre for Greek higher education in the adoption process of quality management.

\section{Vision}

Vision was regarded by the EUA-IEP team as a missing element for most of the universities. One report stated: "a vision of the future of the [University X] must be developed, which can be translated into an associated action plan (five years or longer)". Another team made a similar recommendation: "The Review Team strongly recommends the promotion of a vision for the [University X] related to the development of the $[\mathrm{X}]$ region and the well-being of its people. Such a vision 
should provide a focus for the strategic management plan and quality management".

In contrast, only a few reports noted the presence of a vision for the university. One report, which was able to quote the university's explicit vision, also wrote:

While the mission of [University X], as with all Greek universities, is outlined by law, the university's 'vision' was formulated by the current rectorate and, as stated in the self-evaluation report, it reflects accurately the vision of most members of the academic community of the university.

In a similar vein another report wrote:

The University has, as stated by the Rector, a clear vision and a coherent mid-[to]long term strategy.....all those excellent goals need a well developed set of steps and strategies to be able to develop them within the organization. Nevertheless, we would like to point out the difficulties that the government bodies for the university have to develop a strategic plan for the university. In fact, most of the strategic decisions seem to belong to the Ministry of Education. This lack of autonomy seems to reinforce some peculiar ways of political negotiation between the university and the Ministry, and probably, is one of the reasons for not having strategic plans at the university level. The strategic plan seems to exist implicitly and is therefore conveyed mainly orally.

\section{Decision-making}

In all reports, the decision-making process and structure received direct attention. Frequent mention of this process was made in almost every report. They referred to how Greek law determines the decision-making process; one report stated:

The central decision-making role of the university is very restricted. Indeed, central figures at the university, such as the Rector, and the central bodies of the university, such as the academic Senate, have very restricted spheres of influence. Departments, rather than schools or faculties enjoy a high level of autonomy, while central bodies function essentially to represent those departments. Meanwhile, the official role of the Rector and her/his staff is mainly to execute the decisions of the central bodies. This leaves a gap where strategic decision-making and universitywide development should be taking place.

Another team observed that "State control is visible at all levels of the university ... and moreover, the decision-making procedures are over-regulated". A similar type of argument appeared in another report: 
The fact that university decision-making structures are determined by the government is a significant obstacle to their efficient improvement through innovative new ideas from within ...The major weakness of the management of the university is the dispersed departments, the consequent difficulty of bringing them together, and the apparent lack of incentives for them to cooperate with each other.

\section{Leadership}

The focus on leadership present at each of the universities permeated all of the reports. The team pointed out the complexity of managing a particular university:

The complexity of managing this university can hardly be over-stated, and the review team left with admiration for the way in which the rector, along with his team of vice-rectors and deans, is addressing the challenges. Leading this university requires considerable vision, energy, enthusiasm, as well as great management and negotiation skills. The Team's impression is that the university has the leadership it needs and deserves.

Furthermore, this rector received highly positive notices from the team: "It was apparent that the rector has a vision for the development of the university as a socially responsive, dynamic and research-driven institution, and is keen to push the university in this direction by all means at his disposal". The term "excellent" appeared more than 25 times in this report. In contrast, another report stated: "The rector (and vice-rectors) should give more emphasis to his/their role as academic head of the university. He/they should prepare the Senate's decisionmaking in a transparent way and give a long-term vision with small steps of implementation".

The "vision" for the university lies directly parallel to the leadership the rector provides. The EUA-IEP team made clear that "The success of the [University X] will be determined by the quality of its leadership, by the quality of its staff and by its capacity for free and open discussion to emerging challenges and alternative solutions" and "the rector is a strong unifying force at the top management level".

The mission and the formal decision-making structures and processes of each university, as mentioned earlier, were the same for all universities in Greece. Vision and leadership appeared in these reports either as a strong point or as a special recommendation of the EUA-IEP teams. The qualitative findings in this study were limited to explaining in detail the degree of vision and leadership as factors that influenced the universities to adopt quality management practices. These latter categories received special attention by the review team as they 
considered the principal themes surrounding each university as it introduced change, which is related to the central aim of EUA-IEP, i.e. "to examine the capacity for change in a university by studying its decision making process and organizational structures". These issues appeared under the heading "Capacity for Change". Unquestionably, the mission, vision, decision-making, and leadership of the university are defined as the pivotal factors introducing and motivating modification. In my case, though, the eight universities that participated in EUA-IEP were experiencing multiple types of vision and leadership styles.

Issues such as the department dynamic, research capacity, and the complexity of the Greek universities received specific attention, as presented in the following analysis.

\section{Departments' Autonomy}

The qualitative findings indicated that a department in Greek universities is characterized by autonomy. One report stated:

The university enjoys autonomy in academic matters-as exercised by the departments-so that neither the central bodies of the university nor the state (through the ministries) have an influence on designing the curricula, the distribution of teaching load or assigning teaching methods for undergraduate studies.

In another report the team had written:

There is an autonomy rarely experienced in Europe when looking at the freedom of the professors in not only the content of their research and courses [but also with regard to] the basic idea of academic freedom.

In a similar vein, the following statement appeared in another report:

The university hierarchy is regulated by law and is highly decentralized. Departments have major decision-making powers with regards to their own education and research policies and may make proposals for new positions as well as allocate their part of the budget; they have full autonomy in teaching, curriculum development, the selection of academic staff at all levels (though nominally subject to Ministry approval), and the selection of graduate students.

Another characteristic example of the departmental autonomy was noted: 
The way in which the university made efforts to engage in this process [EAU-IEP evaluation] as an academic community impressed the evaluation Team immensely. However, efforts were evidently more effective in some schools and departments than in others. Indeed, the Team was disappointed that no senior members of [Department $X]$ in particular were available to meet the members of the Team during the main visit. This indicates that although the commitment and engagement of the leadership was assured, there were some difficulties in reaching more deeply inside the institution.

\section{Research Capacity}

The review teams pointed out issues related to the research capacities in almost all cases. The team underscored the strong relationship between teaching and research. In one report, the following was written:

According to the opinion of their students, [University $\mathrm{X}$ ] has reached a good equilibrium between research and teaching in most of their departments. Students consider that having a good research department helps in having good teaching and in some areas, the selection of the [University $\mathrm{X}]$ as their first choice was due to this factor.

Other statements by review teams extolling the importance of research included:

Research performance is excellent ... While it is not the role of the team to judge the academic standards of the [University X], it recognized the very high level of basic and fundamental research activity in the university despite being a relatively young university.

Research is certainly a strength at the [University Z]. The research output of each individual and department is impressive given the relatively poor funding, and is extremely well presented in a major university publication.

This section ended with observations concerning the complexity of the Greek university through the eye of EUA's teams. The topic received major consideration in almost every report. In many cases, the reviewers emphasized how the current complexity of the Greek university is not totally understood. One report wrote:

Higher education institutions in Greece have unique operational conditions and constraints at present that are inconsistent with most of the European universities (such as rules for students, selection and placement, free housing, free books and free meals for periods of six years or more). 
From the above one could grasp the complexity of the Greek university.

\section{Summary}

Table 7-1 summarizes the analysis of the university characteristics. I was able to provide detailed information for each case (university) regarding longevity, size, location, and range of studies in the university. I was unable to categorize universities with regard to their mission, vision, leadership, and decision-making, even though these issues appeared to have received the bulk of the attention in each of the eight reports.

The analysis revealed that four types of universities engaged in the EUA-IEP. Four universities were old, medium-sized, peripheral, and multidisciplinary. Two universities were new, medium sized and multidisciplinary as well. And the remaining two universities were old, urban, and multidisciplinary; one of them was medium-sized and the other large. It is possible that other factors such as vision and leadership were related to the engagement in external evaluation of quality management. However, as mentioned earlier, these reports did not allow me to draw such a conclusion.

Table 7-1 University characteristics

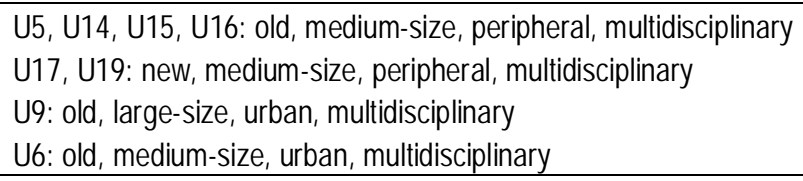

The following section discusses issues concerning the quality management dimensions.

\subsubsection{Quality Management Dimensions}

The qualitative analysis underpinned my expectation that these reports would give evidence to explain the quality management dimensions as discussed in Chapter 4 . There were, however, very different emphases between the reports and significant differences were also evident in quality assessment practices across the eight cases. In this section, I discuss only quality management dimensions and do not make judgment and critique about comparisons and assumptions between the participant universities' quality management performance per se. 
These findings were relevant in explaining the theoretical framework and the nature of quality management within universities that have adopted quality management practices, such as EUA-IEP.

\section{Formalization}

In all reports activities which related to quality management formalization (written documentation in an organization as well as referring to established rules, procedures, job descriptions, regulations, mission statements, and policy manuals) received direct attention by the EUA-IEP review teams. In chronological order, in the beginning of the EUA-IEP process, a review team wrote: "Central to our opinion is that compared to the general trend in Europe, there is a clear lag in development regarding quality strategies at a national as well as an institutional level" and the team made recommendations of what university [X] needs to adopt in terms of quality (i.e. explicit external and internal procedures for evaluation and peer review, students involvement, strategies to improve quality of research and of teaching and learning etc). Later recommendations appeared was:

The review team strongly recommends to design a strategy for the [University $\mathrm{X}$ ] 2010. The University should clearly embark on deciding a strategy for the next ten years: decide the most important objectives and priorities, formalize and communicate them, develop strategies and actions and finally monitor and review performance.

In another report appeared: "an internal law and administrative support is needed for providing an infrastructure to support, promote and enhance the development of a Quality culture and a Quality Assessment system". Also, the review team in another report of 1999 wrote: "There is no institutional organized quality assessment system at university level. An internal regulation guide is in effect for externally funded research and postgraduate studies".

Another report observed: "The evaluation of the quality in the promotion process of academic staff is not systematically implemented". In a comparable vein, an EUA-IEP team discussed that

Until now, there has been no central office responsible for quality improvement and some schools and departments have consequently devised their own systems. The Team now strongly urges the establishment of a central office.

In some reports the review team observed that "much of the data for the self evaluation was gathered for the first time for this evaluation". In other reports the 
team noted "systematic quality assurance procedures are not yet developed in Greece and there is not normally formal mechanism of quality management in the universities.

Some reports stated that "methods for systematic self-assessment are needed", implying that this is not formalized yet. Moreover the team noted that "the statistics which were provided for the Team were sparse and not up to date. It was clear too that they were not in use in the day-to-day management of the University". The formalization of quality management in the university, as evidenced by the EUA-IEP reports seems that it was not a top priority in universities during this period.

\section{Centralization and Complexity}

Departments are largely autonomous within Greek universities and in many cases they developed their own quality assessment procedures. For example, one report stated:

Quite a number of departments have developed quality assessment procedures for their courses in which students participate....the university has no centralized quality assessment of courses, which means that there is not necessarily a followup of any of the problems revealed by quality assessment.

As another team pointed out, "there is a high degree of decentralization in the academic management of the University so that each faculty makes its own priorities and thus it is very difficult for the University to act as an entity".

In another report the review team wrote:

From the discussion in the senate the audit team understood that some feel that such a planning mechanism [quality in the university's strategy] might be a danger for the academic autonomy of the individual staff members. In this report was tried to explain the contrary in fact holds true: developing teaching and research in a autonomous way presuppose the existence of a framework on the institutional level. If no such framework exists initiatives of individuals may easily fail to succeed because they have no guidelines to go by.

Apart from the individual autonomy that one review team considered "the autonomy of the university as a whole" it suggested that "this [autonomy] is much better guaranteed if it has a mechanism for quality assurance in operation and can show to the outside world that it is taking the quality of its operation seriously". 
Later (in chronological order), some reports contained statements such as: "Some departments use student's questionnaires but, according to the students, there is no follow up to the process". In a similar vein another report wrote: "quite a number of departments have developed quality assessments procedures for their course in which students participate". In another report also the review team defined that "student questionnaires are used sporadically".

As I focus on visits occurring during the period 1999 to 2005, I could not fully document in the data collection process specific evidence to support growth in self-assessment undertaken in specific university departments. Although I have personal communications indicating the direction on the part of specific individuals toward self-assessment is positive, external data does not yet support that movement. The above shows a high level of decentralization rather than centralization. Also, it shows that quality management is seen mainly as student evaluations of individual courses without assured follow-up, implying that quality management in these universities is neither very sophisticated nor complex.

\section{Resources}

In terms of staff allocation, we can readily see the number of academic or administrative staff that participated in order to produce the self-assessment reports. In one report, there appeared the following statement:

The EUA evaluation panel appreciated the work carried out by the university's self-evaluation group, and indeed by the whole university community in contributing to the self-evaluation report.

Budgets were not directly connected with this analysis. Nevertheless, the EUA website presents information concerning resources needed for the self-evaluation process, namely the required fees. These charges pointed to the amount of monetary resources a university is willing to allocate for this particular process. During the EUA-IEP progression, universities covered their fees either through budget allocation or from funding received through the Ministry of Education. However, human and financial resources were not found in regards to general quality management practices. The review team, in the majority of the reports, suggested the development of a quality assurance or a quality management system. For example, one report stated: "The Team now strongly urges the establishment of a central office, with its Director reporting directly to the Rector. The office would be responsible for a range of monitoring and improvementoriented activities". 
Without mature quality management in these universities, I was unable to provide evidence from these reports regarding the more detailed elements of quality management systems such as Aim and Scope, which were mentioned in chapter 2 .

\subsection{Analysis in Comparison to the rest of the 21 Universities}

This section presents a comparison of the eight universities that underwent an EUA-IEP evaluation with the 13 universities in Greece that did not participate in the EUA evaluation process. A comparison with participants and nonparticipants could be made solely on the following characteristics: age, size, location, and range of studies. The veracity of the data presented may be confirmed by visiting the various university websites or the Ministry of Education's website. To maintain anonymity, I encoded each university with a number. Table 7-2 provides information regarding the characteristics of the nonparticipant universities.

Table 7-2 Characteristics of the 13 non-EUA-IEP universities

\begin{tabular}{|c|c|}
\hline Types of categories & $\begin{array}{c}\text { Non EUA-IEP } \\
\text { universities }\end{array}$ \\
\hline Type Alfa (A): old, small, urban, monothematic & U1, U3, U13 \\
\hline Type Gamma (Г): old, medium, urban, monothematic & U2, U7, U10, U12 \\
\hline \multicolumn{2}{|l|}{ Type Delta $(\Delta)$ : old, medium, urban, multidisciplinary } \\
\hline Type Epsilon (E): old, large, urban, multidisciplinary & U11 \\
\hline Type Zeta (Z): old, small, peripheral, monothematic & U8 \\
\hline \multicolumn{2}{|l|}{ Type Heta (H): old, medium, peripheral, multidisciplinary } \\
\hline Type lota (I): new small, peripheral, multidisciplinary & U4, U18, U20, U21 \\
\hline Type Lambda $(\Lambda)$ : new, medium, peripheral, multidisciplinary & \\
\hline
\end{tabular}

Table 7-3 presents an overview of the EUA-IEP adopters, the non-adopters, and the total population of the Greek universities. Again, letters in the Greek alphabet were chosen for each category.

Tables 7-4, 7-5, 7-6, and 7-7 display the comparison between EUA-IEP adopters and non-adopters, and the university's characteristics influencing the EUA-IEP adopters. 
Table 7-3 Types of universities by participating or not-participating in the EUA-IEP until 2006

\begin{tabular}{lccc}
\hline \multicolumn{1}{c}{ Type } & EUA & non-EUA & Total \\
\hline Alfa $(A)$ & - & 3 & 3 \\
Gamma $(\Gamma)$ & - & 4 & 4 \\
Delta $(\Delta)$ & 1 & - & 1 \\
Epsilon $(E)$ & 1 & 1 & 2 \\
Zeta $(Z)$ & - & 1 & 1 \\
Heta $(H)$ & 4 & - & 4 \\
lota $(I)$ & - & 4 & 4 \\
Lambda $(\Lambda)$ & 2 & - & 2 \\
& ---- & ---- & --- \\
Total & $\mathbf{8}$ & 13 & 21 \\
\hline
\end{tabular}

Table 7-4 By age

\begin{tabular}{|c|c|c|c|}
\hline & \multicolumn{2}{|c|}{ University Age } & \multirow[t]{2}{*}{ Total } \\
\hline & Old & New & \\
\hline EUA & 6 & 2 & 8 \\
\hline Non-EUA & 9 & 4 & 13 \\
\hline Total & 15 & 6 & 21 \\
\hline
\end{tabular}

Table 7-5 By size

\begin{tabular}{lcccc}
\hline & \multicolumn{3}{c}{ University Size } & Total \\
\hline & $\mathrm{S}$ & $\mathrm{M}$ & $\mathrm{L}$ & \\
EUA & - & 7 & 1 & 8 \\
Non-EUA & 8 & 4 & 1 & 13 \\
\hline Total & $\mathbf{8}$ & $\mathbf{1 1}$ & $\mathbf{2}$ & $\mathbf{2 1}$ \\
\hline
\end{tabular}

Table 7-6 By location

\begin{tabular}{lccc}
\hline & \multicolumn{2}{c}{ University Location } & Total \\
\hline & Urban & Periphery & \\
EUA & 2 & 6 & 8 \\
Non-EUA & 8 & 5 & 13 \\
\hline Total & 10 & 11 & 21 \\
\hline
\end{tabular}

The majority of the EUA-IEP non-participants were monothematic universities (7 out of 14, Table 7-7). By contrast, all of the universities characterized as old, medium, peripheral or urban, and multidisciplinary had participated in EUA 
evaluation (5 out of 5) (Table 7-3, type $\mathrm{H}$ ), as well as all of the universities characterized as new, medium, peripheral and multidisciplinary. One of the two old universities that was large, urban, and multidisciplinary had participated as well in the EUA-IEP process. The majority of the non-EUA participants were old universities (Table 7-4). Also, the seven monothematic universities (Table 7-7) did not participate; they were spread across the other characteristics: either urban or peripheral and small or medium (8 out of 13). The four new, small, peripheral, and multidisciplinary universities followed this trend. As mentioned earlier, none of the EUA-IEP cases were located in Athens (8 out of the remaining 13).

Table 7-7 By range of studies

\begin{tabular}{lccc}
\hline & \multicolumn{2}{c}{ Range of studies } & Total \\
\hline & Monothematic & Multi \\
EUA & - & 8 & 8 \\
Non-EUA & 7 & 6 & 13 \\
\hline Total & 7 & 14 & 21 \\
\hline
\end{tabular}

\subsection{Summary and Conclusion}

The idea of the present study was to detect relationships between organizational factors for stability or change that influence the adoption (or lack of adoption) of quality management at the meso level in Greek higher education. Eight out of 21 universities undertook the EUA-IEP until 2006. Therefore in this chapter in order to obtain an indication of change, I compared two populations of universities, the EUA-IEP participants and the non-participants.

Evidence from the reports indicated that quality management as a formalized practice was absent in all 8 universities that participated in the EUA-IEP. In addition data from the reports demonstrated that centralization and complexity were absent as well. The majority of the reports showed that there were some activities in regards to student's evaluations but without formalized follow-up activities. Student evaluation activities were organized in individual departments which represented the autonomous status of Greek academic departments.

The results of the qualitative content analysis identified organizational factors that were capable of influencing the adoption of quality management. The data were collected from eight evaluators' reports associated with the eight universities that voluntarily took part in the EUA-IEP. The reports yielded observations on three themes: isomorphic pressures, university characteristics, 
and quality management dimension. Coercive pressure did not play a significant role in the decision to invite the EUA-IEP since this action was made voluntarily by the university rector and approved by its Senate. Besides, the EUA-IEP processes took place in a context before the quality assurance law came into force. All reports consistently wrote that there was no pressure from the state for adopting quality management practices.

In DiMaggio and Powell's (1983, 1991) writings, the distinction between normative and mimetic is not clearly identifiable; especially in empirical studies where the researcher needed to have clear evidence in order to categorize isomorphism. The EUA-IEP reports did not provide clear statements that directly pointed to external pressures; conclusions about normative and mimetic pressures must therefore be drawn through indirect inferences.

Normative pressure was argued to have played a role in this study. Normative pressures stem primarily from professionalization. Here, professionalization was indicated by the fact that the EUA-IEP self-assessment reports suggested normative pressure, especially by their rectorate's management commitment and by the fact that these universities participated in a professional association, i.e. the EUA. The eight universities that participated in this evaluation practice were seeking feedback about their overall performance. An alternative chain of argument that normative pressure might influence a university's participation in the EUA-IEP may be found in the communication and affiliation of specialists in professional networks (van Vught, 1989 in Maassen \& Potman, 1990, p. 406). Nilsson et al. (2002, p. 13) pointed out that "the value of peer review is a central assumption of the review program [EUA-IEP]. The program relies upon a relatively small, close-knit cadre of rectors and ex-rectors (the majority) to form the review teams". Normative pressure was related to the peer review team's profession, because Greek rectors, who decided to participate in this action, felt probably more "secure" with the idea that international colleagues performed the review process. However, absence of setting policies and goals in terms of quality, absence of treating quality as a strategic variable in the universities, as well as the absence of human and financial resources to develop quality management activities are associated with the notion that quality management in Greek universities during 2005 was not a routine practice. Clearly, quality management was not a function of the universities' quality performance and therefore the argument that participation at the EUA-IEP related to normative pressure remains unclear.

The argument that participation in the EUA-IEP might be associated with mimetic pressure is based on one of the university characteristics: location. All 
EUA-IEP participants were located outside of Athens. Thus, theoretically speaking, these universities would desire participation in the EUA-IEP to validate the "quality" of their institution. It is possible that Athenian universities were perceived as "elite" within the Greek higher education system. Banta (1993) noted that pioneer universities in quality assurance practices were not from elite universities. This was found for the EUA-IEP also, when Nilsson et al. (2002, p. 12) reported that "few elite universities apply for the programme". Perhaps universities on the periphery of Greece wanted to use the EUA-IEP as a compensating policy to gain prestige or legitimacy. It was noted before that governmental decision-making for resources as well as students' preferences were in favor of Athenian universities. Practices such as the EUA-IEP may initially have been adopted to gain prestige and to show that "the sleepy peripheral university was becoming more Europe-minded" (paraphrasing DiMaggio \& Powell, 1991, p. 70). Knowing that at least some of the EUA-IEP participants promoted the report about their university with the letterhead of the EUA (e.g. bringing it to meetings with decision-makers and colleagues, or putting on the website) could be interpreted as a sign of trying to establish legitimacy through mimetic isomorphism.

Monothematic universities seemed reluctant to engage in the EUA-IEP. However, the majority of monothematic universities were old and mostly located in Athens (6 out of 8 ) and possibly the more parsimonious explanation lies in their following the "elitist" Athenian attitude towards quality management. Furthermore, monothematic universities were small (4 universities) and medium sized ( 3 universities). These universities may have lacked complexity in their organization. Possibly then, they felt less pressure to adopt formalized quality management practices. In regards to all EUA-IEP participants, the majority of the 191 universities, until 2009, were multidisciplinary universities (personal communication with EUA, V. Atanassova, 14-11-2008).

One of the premises of the quality management literature, especially of the EUAIEP, is that leadership is crucial in the adoption of quality management. Leadership was the other important university characteristic that seemed to correlate with having participated in the EUA-IEP. Mission and decision-making were the same for all 21 universities, and vision was regarded by evaluators' teams as a missing element for most of the EUA participants, which might indicate a low stage of development of institutional leadership. Nevertheless, the leadership, as noted, appeared to play an important role in the decision to take part in the EUA-IEP. Leadership and vision were considered by EUA evaluation teams as the principal themes surrounding each university in the introduction of change. It is possible that leaders in Greek universities were motivated to 
participate in the EUA-IEP as a result of their profession (normative isomorphism) and they saw this as an occasion to procure external support for changes they wanted to make to the vision (and practices) of their institutions.

Nevertheless, it seemed that the normative and mimetic pressures alone were insufficient requirements to initiate changes in two thirds of the 21 universities. Since initiation of change is documented in the data, the leadership perspective (chapter 8) deserves a closer examination as another resource for the adoption of quality management at the meso level in Greek higher education. 


\section{Surveying of Quality Management via Malcolm Baldrige Education Criteria}

\subsection{Introduction}

Chapters 6 and 7 of the book shed light on the quality management and isomorphism at the macro and meso levels in Greek higher education. Their common denominator was the document-based analysis of the adoption of quality management, i.e. through newspapers articles and evaluators' reports. The aim of the current study was to go beyond documents and gather knowledge on how leaders (rectors and vice-rectors) in Greek universities perceived neoinstitutional pressures and to what extent they adopted quality management practices to improve their university's performance. The quality management literature had suggested that quality practices and concepts can be effective only if properly supported by top administrators. Rectors and vice-rectors (three or four in each institution) form the leadership in Greek universities. Learning from the "top" and their experiences with isomorphic pressures that influenced universities regarding quality management must be a valuable source of information. There were no published instruments known to assess the organizational factors that influenced the adoption or non-adoption of quality management in Greek higher education. To answer the quality "puzzle" in Greek universities I used a questionnaire derived from the U.S. Malcolm Baldrige National Quality Award Criteria for Performance Excellence in Education (hereafter simply MB) (NIST, 2005).

\subsubsection{Methodology}

A survey method was chosen for quantitative and qualitative data collection in this study. The purpose of this tool was twofold: to gather data (descriptive data on the university's characteristics), and to understand the isomorphic pressures on adoption or otherwise of quality management at the meso level in Greek higher education. I used the MB as a survey tool, which is mostly a US-based model, and not other models such as the fairly similar EFQM, even though my empirical sample is located in Europe. The reasons for this decision are as follows: first, the development of MB-assisted educational institutions with self-assessment began in 1995, giving this instrument a long track record. Second, sources regarding MB were more accessible than EFQM; the US National Institute of Standards and 
Technology (NIST) website 22 offered almost all information regarding this award, more than the website for the EFQM ${ }^{23}$ did. Finally, the MB had been piloted and then used for higher education (Seymour, 1996) and therefore, the literature was richer with published practices from award recipients. Articles, dissertations, and books (Blazey, et al., 2003; Moore, 1996; Ruben, 2004; Sorensen et al., 2005; Wysong, 2000) were other resources that helped me to understand this model.

\section{Survey Instrument}

While quality management in this study was understood as a process for performance improvement, the $\mathrm{MB}$ criteria on the one hand, enabled researching a leader's perception in relation to the pressures derived from the neoinstitutional literature, while on the other hand, those criteria enabled collection of specific data about the extent to which quality management processes were being implemented at the meso level. At the time of this dissertation, some studies investigated leadership behaviors in higher education that practiced quality principles and the MB (Wysong, 2000). Other studies emphasized that the MB provides a valuable gauge of organizational effectiveness (Ruben, 2007).

Each statement (item) in the survey emphasized factors that were derived from the seven MB categories, shown in Table 8-1.

Table 8-1 The Seven MB Categories

\begin{tabular}{|ll|}
\hline 1. & Leadership \\
2. & Strategic Planning \\
3. & Student, Stakeholder, and Market Focus \\
4. & Measurement Analysis and Knowledge \\
& Management \\
5. & Faculty and Staff Focus \\
6. & Process Management \\
7. & Organizational Performance Results \\
\hline
\end{tabular}

Source: MBNQA 2005, NIST

The first part of the survey asked for information about the university's characteristics-age, size, range of studies, and location of the universitythrough open questions. In addition, it included question (A4) such as "Do you have any quality assurance system?" which required "yes or no" responses (categorical scale).

\footnotetext{
22 See www.nist.gov.

${ }^{23}$ See www.efqm.org.
} 
The second and main part of the questionnaire included 73 statements based on the $\mathrm{MB}$ criteria to evaluate the importance and implementation rate of the $\mathrm{MB}$ criteria, and functioned as a tool in comprehending how universities perceive neo-institutional pressures. The most important constructs consisted of multiple measures to ensure accuracy and comprehension.

Each of the seven MB categories was subdivided into a number of statements. For each statement, two categories were formatted with a dual scale using a 1-10 (1 was "none", 10 was "highest"): the extent to which the rector or vice-rector regarded this statement as important (the importance rate) and the extent to which the rector or vice-rector believed that the university implemented this practice (the implementation rate).

The questionnaire was clustered under seven categories corresponding to MB. The categorical questions were generated according to Professor Ruben's feedback in his e-mailed response (March 2005). Table 8-2 lists the MB categories and the total number of questions cited in the survey tool per MB category.

Table 8-2 MB Categories and total number of questions cited in survey tool

\begin{tabular}{lc}
\hline \multicolumn{1}{c}{ MB Categories } & Total number of questions \\
\hline 1. Leadership & 9 \\
2. Strategic Planning & 7 \\
3. Student, Stakeholder, and Market Focus & 9 \\
4. Measurement Analysis and & 17 \\
$\quad$ Knowledge Management & 15 \\
5. Faculty and Staff Focus & 7 \\
6. Process Management & 9 \\
7. Organizational \& Performance Results & 73 \\
\hline Total & \\
\hline
\end{tabular}

In the last part of the questionnaire, I provided the respondent rectors and vicerectors the chance to make extra comments. The actual survey instrument is located in Appendix (B1 Greek questionnaire, the original version being followed by the English translation in Appendix B2).

\section{Validity and Reliability Issues}

Following Wysong's (2000) procedure, in order to establish content validity of this instrument, I asked Professor Ruben (Ruben, 1995, 2004, 2006, 2007) as an 
expert in the MB field to evaluate it (personal communication, March 2005). He made suggestions regarding the content and also correlated the survey items within MB categories. I made modifications to the questionnaire based on the feedback from this field expert.

Since this study took place in Greece, the items used in this survey were translated into Greek. To ensure the quality of the translation, it was checked by a professor in sociology and a professor in Greek literature. Finally, the text was adapted according to expert comments. The revised instrument was then used to conduct a pilot study according to Creswell's (2003) guidelines. The questionnaire was piloted in May 2005 with three former rectors and vice-rectors in Greek universities, known to be among the best-informed on quality management in academia. All completed the survey in 45 minutes. Translation adequacy was evaluated using a focus group of individuals reflecting the population under investigation. I did not have to make any further changes to the questionnaire, based on the positive feedback of the pilot study members.

\subsection{Findings}

\subsubsection{Sample}

The population of the survey consisted of the 20 universities listed at that time by the Greek Ministry of Education and Religious Affairs and that were in actual operation. The target respondents (homogeneous sample) were the rectors and vice-rectors of these universities, in order to exclude possibly confounding the variables (Kerlinger, 1973). In total, 65 individual respondents (rectors, vicerectors, presidents and vice-presidents) were asked to complete the questionnaire. From twenty universities, nine responded within a six-month period, and two wrote to refuse to participate in this research because the law regarding quality management systems was at that time under discussion. From the nine universities, one rector asked me if it was possible to have a face-to-face interview to complete the survey. I was able to collect his answers and thoughts regarding the survey statements which might serve as an explanation later in this study. To increase the return rates I applied Dillman's (2000) follow-up suggestions. I made several personal phone calls in November-December 200524. Ultimately this did not result in any further response. Eventually, the nine out of 20 universities

${ }^{24}$ Dillman (2000) suggests a four-phase administration process. However, I used mostly phones calls. Personal experience indicated that phones calls provide a better response rate than letters, because letters might be filtered out by secretarial staff. 
replied providing a response rate of $45 \%(9 / 20)$. The individual response rate was $14 \%(9 / 65)$.

Responses from only nine universities may not allow for generalization. It has to be realized, however, that Makridakis et al. (1997, p. 400) were satisfied with a $15 \%$ response rate in their survey among Greek CEOs: "the response rate is good for mailed questionnaires, in particular since it required more than one hour for completion and necessitated time from extremely busy people like CEOs or other executives". Besides, I had to take into account that the survey took place in May 2005. As shown in chapter 6, the years 2005 and 2006 in general, and May 2005 in particular, were not auspicious for studying quality assurance in Greek higher education. But Greece, as noted previously, is an environment in which it is notoriously difficult to conduct empirical social science research.

\subsubsection{Response Bias}

Response bias is the effect of non-responses on survey estimates (Fowler, 1998). Bias means that if non-respondents had replied, their responses would have substantially changed the overall results of the survey. Creswell (2003) suggested that an alternative check for response bias is to contact by phone a few nonrespondents and determine if their answers differed substantiality from those of the respondents. This technique included a respondent/non-respondent check for response bias. I followed this suggestion but was unable to receive answers that would have helped to establish bias or its absence.

As a proxy non-response analysis, it may be useful to note the relation of respondents and non-respondents to EUA-IEP universities versus non-EUA-IEP universities. Table 8-3 shows that the survey was not so popular in the EUA-IEP universities, and there were a greater number of non-responses from them.

Table 8-3 Response rates of EUA participants and non-EUA universities

\begin{tabular}{lccc}
\hline & EUA & non-EUA & Total \\
\hline $\begin{array}{l}\text { MB Respondent universities } \\
\begin{array}{l}\text { MB Non-respondent } \\
\text { universities }\end{array}\end{array}$ & 3 & 6 & 9 \\
\hline Total & 5 & 6 & 11 \\
\hline
\end{tabular}

I expected leaders from EUA-IEP universities to be more sensitive to the issue of quality management practices and more interested in participating in this type of survey. Unfortunately, the first finding of this survey was antithetical to my 
expectations. The disadvantage is that these findings do not allow me to triangulate the data. In contrast, wider coverage of data across all universities rather than just the EUA's eight cases allows me to build a better picture of the whole higher education landscape in Greece. Thinking back to the low maturity of quality management processes in universities that participated in the EUA-IEP, in the previous chapter, the likelihood of finding a bias towards low maturity of quality management practices in the non-EUA-IEP universities seems limited.

\subsubsection{Dealing with People who "Don't Know"}

Missing data in this survey dealt with a "no opinion" option, as the survey instrument included the following explanation for respondents: "if you do not have an opinion please leave the statement without a check". This option was followed by a note stating that if the respondent had any comments to enter them in the last part of the survey. Fowler $(1995, \mathrm{p} .165)$ stated that when respondents are asked for the opinions or perceptions of things beyond their direct experience, a "don't know" response is a potentially meaningful answer, not missing data, and it is best obtained in an explicit, standardized way. Creswell (2003, p. 189) suggested that researchers contact individuals to determine why they did not respond. In my study, I followed this suggestion.

In this survey tool, the "missing data" aspect was absent by design. With regards to "no opinions" concerning the different items, the survey instrument did not include separate tick boxes for that, and respondents were instructed: "if you do not have an opinion, please leave the statement blank". At the same time, respondents were asked to enter any remarks they might have in the open response boxes in the last part of the survey. Fowler (1995, p. 165) stated that when respondents are asked for the opinions or perceptions of things beyond their direct experience, a "don't know" (or no opinion) response is a potentially meaningful answer, not missing data. I surmised from the returned questionnaires that the total amount of "no opinion" answers was $8 \%$ of the importance rate items and $9 \%$ of the implementation rate items. Tables B1 and B2 in the Appendix present the MB categories mentioning explicitly the "no opinions". The data show that amongst the nine universities, the "no opinions" appeared more frequently in five universities and in similar proportions for both importance and implementation scale items. In two of these universities, the "no opinion" items even rose to $22 \%$ and $30 \%$ of the all survey items in these two scales. In the other three of the five universities most "no opinion" responses related to the MB Category 4 "Measurement, analysis and knowledge management". Category 4 was directly connected with quality management issues. In computing the data for these questionnaires, I substituted both 
importance and implementation with a score of " 1 " (the lowest score). Three respondents inserted remarks that helped to justify this interpretation for imputing this low value to be substituted, e.g.:

To many questions I gave answers; however, I did not check an answer because these processes are informal and directly connected with the personality and the philosophy of the university leadership.

In relation to the implementation rate, such a missing score was understandable (informal process). With regard to the importance rate, it was not clear why a leader did not choose any score from 1 to 10 but rather noted that these processes were connected with the leadership personality and philosophy. This comment did not add to understanding this leader's views on the importance of quality management.

\subsubsection{University Characteristics}

The first part of the survey provided information regarding the university's characteristics: age, size, location, type of studies. This first section presents these characteristics followed by quantitative (QUAN) analysis that related to $\mathrm{MB}$ categories.

Age

The study's participants fell into two age categories: old and new. Of the nine universities, eight were old and one was new.

Size

The study's participants spanned all three size categories: small, medium, and large. Of the nine universities, four were small size universities, four were medium size universities, and one was a large size university.

\section{Location}

The study had both urban and peripheral respondents. Of the nine universities, six were urban universities and three were peripheral universities. There were more urban than peripheral universities, and four of the urban universities were located in Athens.

\section{Range of Studies}

Of the nine universities, five were monothematic universities and four were multidisciplinary. 
To maintain anonymity, I encoded each university with a number (U1-U9). Table 8-4 provides information regarding the characteristics of each participant university.

Table 8-4 Characteristics of participants' universities

U1, U3: old small-size urban monothematic university
U2, U7: old medium-size urban monothematic university
U4: new small-size peripheral multidisciplinary university
U5: old medium-size peripheral multidisciplinary university
U6: old medium-size urban multidisciplinary university
U8: old small-size peripheral monothematic university
U9: old large-size urban multidisciplinary university

The central research question examined the factors influencing the adoption of quality management in Greek universities through 2006. Thus, I posed the question A4 cited in the first part of the survey. All responding universities (9 out of 9) answered negatively to this question: all leaders said that their university did not have a quality assurance system. Besides, I did not receive any responses to the open questions, needed to complete the second qualitative (QUAL) part of this study as I had planned. Thus, although I had planned to utilize a quantitative and qualitative (QUAN $\rightarrow$ QUAL) sequential mixed methods design, this study in the end had to follow a quantitative design.

\subsubsection{Greek Leaders Assess the Malcolm Baldrige Award Education Criteria}

This section provides a descriptive overview of the MB survey results. As noted, quality management was understood as a strategy for performance improvement and the MB categories operated as a tool for university self-assessment and for improving organizational performance. The survey has questions relating to independent and dependent variables of this study. Consequently, the importance rate of leader's perception on MB criteria and categories enabled understanding the degree of pressure that these leaders felt, and accordingly, what type of quality management they perceived as important. The implementation rate represented the university's involvement in quality management through their leader's knowledge.

For each of the seven categories the mean score across respondents per university was calculated across all items both for importance and implementation. The means are reported for both categories. Data showing the mean scores for each participating university was then developed and reported. This was done by 
calculating combined means for each university and for each MB category. Gap scores (importance rate minus implementation) were calculated for each university. The data collection and analysis provided three types of information (importance and implementation rates as well as difference/gap). Table B3 in the Appendix presents overall participants' responses for each of the 73 items covered in the second part of the questionnaire. All items were measured on a 110 point scale; the "no opinion" value was counted as " 1 " as explained in section 8.2.3.

\section{Importance Rate (Isomorphic Pressures)}

The overall importance scores of the survey ranged from a high of 10 (U6) to a low of 3 (U5). Notably, the latter university (U5) responded "no opinion" for 22 items. The high scores suggested high isomorphic pressures on the universities. These findings perhaps suggested priorities for these nine universities. Category 1 "Leadership" showed the highest pressure on the nine leaders, followed by category 5 "Faculty and Staff Focus". Category 4, "Measurement Analysis and Knowledge Management", appeared to be the category were the lowest pressure was felt. These findings suggest how the Greek leaders assessed the MB criteria and reveal the degree of pressure that they felt and how they prioritized their needs.

\section{Implementation Rate (Application)}

How leaders prioritize their strategy was further elaborated through the respondents' implementation scores. Whereas the importance rate indicated their feeling of pressure, the implementation rate indicated the actual degree of adoption of quality management practices for the university's performance improvement. The implementation rate was lower than the importance rate within universities in all MB categories. The exception was that U4 and U5 scored equal in importance and in implementation rate in two categories: "Process Management" and "Organizational Performance Results". These two universities, as noted, were the two universities with the most "no opinion" answers.

Figure 8-1 depicts the overall results for the MB categories. It compares the mean scores which were derived from the Greek leaders' perception (importance and implementation). The highest-scoring category regarding the implementation rate status was Category 6 "Process Management". Implementation scores indicated how the university reacts to pressure. "Measurement Analysis" and "Strategic Planning" were in last place. These finding suggested that the responding universities paid attention mostly to "Process Management" and least to "closing loop' from process management back to the leadership's analysis and strategy. 


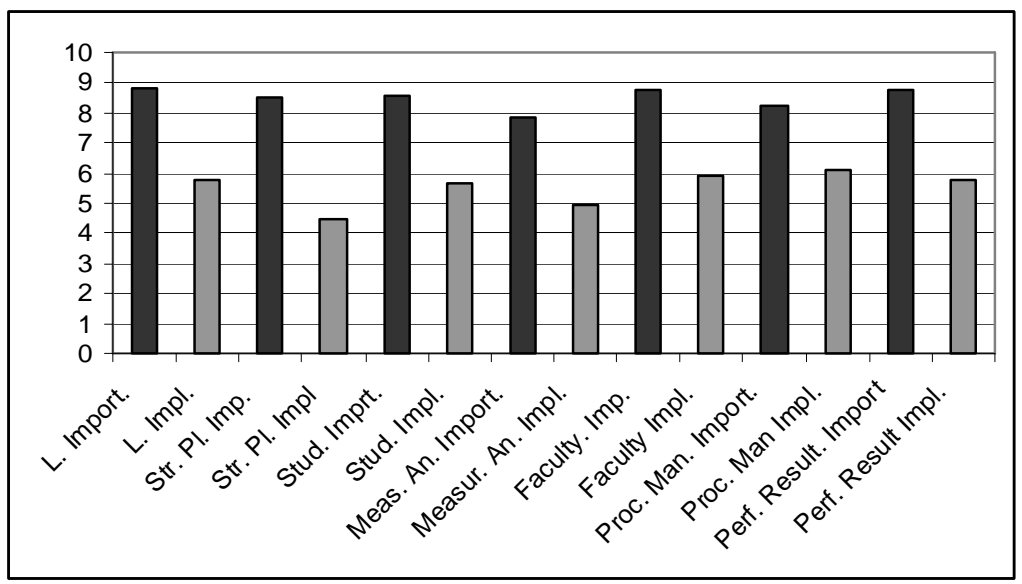

Figure 8-1 Importance and Implementation rates (averages of 9 universities)

\subsubsection{Hypothetical Malcolm Baldrige Scores for Greek Universities}

Blazey et al. (2003) stated that the MB criteria, together with the scoring guidelines are to help examiners identify the key strengths and vital areas needing improvement in organizations that they are evaluating. This will help leaders focus their efforts on the steps needed to reach the next developmental stage. A score of 0 to 200 will be interpreted as indicating that the university is in the very early stage of a transition from reacting to problems of general improvement in orientation evidence; I call this a 'lacking' quality management system. A score of 200 to 400 indicates that many areas need work. The beginning of a systematic approach to the evaluation and improvement of key process is evident, and that perhaps there is a commitment to quality in this university ('embryonic' quality management will be the term used for this situation). A score of 400 to 600 indicates that the approach is well deployed ('developing'), although deployment may vary in some areas. A fact-based systematic evaluation and improvement process is in place for improving the efficiency and effectiveness of the key process. A score of 600 to 800 indicates that there is evidence of an effective systematic approach in place ('advanced' stage of quality management). The approach is well deployed, with no significant gaps. There is clear evidence of refinement, innovation, and improvement integration as a result of organizational-level analysis and sharing. Finally, a score of 800 to 1000 indicates that there are effective systems in all areas of the university. This score indicates that there exist good integration and good to excellent results in all areas (an 'excellent' quality management system). 
I hypothesized that the survey results regarding implementation rates could be interpreted as such an $\mathrm{MB} \mathrm{score}^{25}$, to give me another picture of quality management at the meso level of Greek higher education. The MB implementation mean score (e.g. leadership mean score in university $X$ equaled 7.6 or $76 \%$ of the maximum) was multiplied with the assigned points for each of the $\mathrm{MB}$ category (e.g. MB leadership could be assigned 120 points). In the example, university $X$ would be awarded 91.2 points in Category 1 $(76 \%$ x 120 =91.2). Table B4 in the Appendix presents the MB category scores and the overall quality index score.

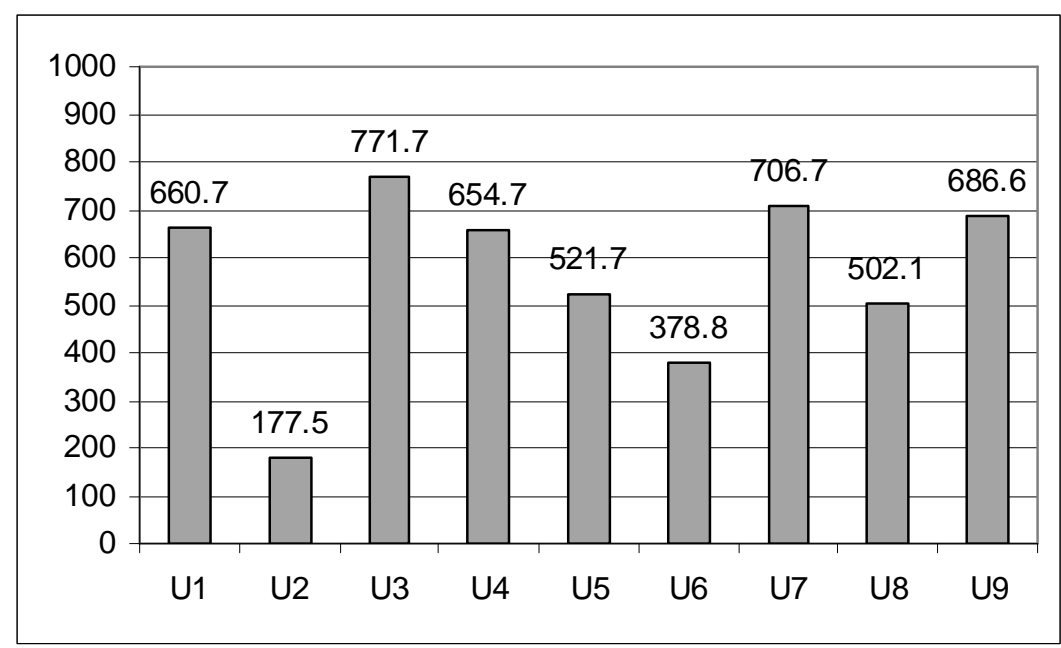

Figure 8-2 Hypothetical quality index for Greek universities

Figure 8-2 presents these scores as a diagnostic assessment system for each of the nine universities, in order to go beyond their initial negative answer that they "do not have any quality assurance system". University 2 appeared in the category between 0-200 points ('lacking' quality management). University 6 reached between 200-400 points, which specifies that the beginning of a systematic approach to evaluation and improvement of key processes is evident ('emerging'). Two universities (5 and 8) appeared in the category 400-600 points-"the approach is well deployed, although deployment may vary in some areas or work units" ('developing' quality management). And the five other universities achieved a score of 600-800, which indicates there is an evidence of an effective systematic approach in place ('advanced' quality management). None of the nine universities found that there

${ }^{25}$ For this technique I followed Ruben's (2005) readings. 
are effective efforts in all areas of the university, which would have been evidenced by a score between $800-1000$.

\subsection{In-depth Analysis: Construction of Diagnostic Items}

The next step in the analysis included discovering relationships between independent and dependent variables. Before starting it has to be noted that this questionnaire does not address coercive pressure ${ }^{26}$. Consequently, normative and mimetic were the only isomorphic pressures that were covered by $\mathrm{MB}$ instrument. Appropriate survey items were chosen; they were used no more than once and, since this is about pressures rather than practices, the variables were derived from the importance rate questions.

\subsubsection{Neo-Institutional Pressures}

\section{Normative Pressure}

A variable for normative pressure in this questionnaire is linked with several items and categories: items on benchmarking and on communication of quality management practices, Category 3 "Student Stakeholder and Market Focus", and Category 5 "Faculty and Staff Focus" (detailed information in the Appendix B3). It merits attention that during this approach, the normative pressure diagnosis was constructed from a combination measurement (mean score).

\section{Mimetic Pressure}

Mimetic pressure diagnosis is linked with two survey items, one from Category 2 "Strategic Planning" and another from the Category 4 "Measurement Analysis and Knowledge Management". The reader again can find detailed information in the Appendix B3 under the term "mimetic pressure".

The challenge of this part was to identify and describe the isomorphic pressures in a robust yet sensitive way. I recoded the data into three categories. I divided the possible scores into three pressure categories (low, medium, and high) of equal 'length'. Scores between 1.0 and 3.3 meant that there was low pressure. Scores between 3.4 and 6.6 suggested medium pressure. Scores between 6.7 and 10 suggested that the pressure was high. Tables 8-5 and 8-6 show the overall level of normative and mimetic pressures translated into the pressure scale. Almost every leader reported high pressure. U4 and U5 seem to feel lower pressure than the other universities. U4 scored "no opinion" regarding mimetic pressure.

\footnotetext{
26 On May 30, 2005 the quality assurance law was submitted to the Greek Parliament for discussion and had not yet come into force.
} 
Table 8-5 Normative pressure

\begin{tabular}{lccccccccc}
\hline Normative & U1 & U2 & U3 & U4 & U5 & U6 & U7 & U8 & U9 \\
\hline $\begin{array}{l}\text { Combined mean } \\
\text { score }\end{array}$ & 8.7 & 7.7 & 9.5 & 7.1 & 5.4 & 10 & 8.9 & 9.9 & 9.8 \\
\hline Pressure scale & High & High & High & High & Medium & High & High & High & High \\
\hline
\end{tabular}

Table 8-6 Mimetic pressure

\begin{tabular}{lccccccccc}
\hline Mimetic & U1 & U2 & U3 & U4 & U5 & U6 & U7 & U8 & U9 \\
\hline $\begin{array}{l}\text { Combined mean } \\
\text { score }\end{array}$ & 9.0 & 9.5 & 9.5 & 1.0 & 7.5 & 10 & 8 & 10 & 9 \\
\hline Pressure scale & High & High & High & Low & High & High & High & High & High \\
\hline
\end{tabular}

Due to the virtual absence of variations in normative and mimetic pressures further analysis was not performed.

\subsubsection{Leadership}

The second part of the survey provided information regarding the university's leadership. In chapter 2 (section 2.2.2), I called Leadership (Category 1), Strategic Planning (Category 2), and Student, Stakeholder, and Market Focus (Category 3) together the 'leadership triad'. These categories emphasized the importance of a leadership focus on strategy, students, and stakeholders. Implementation rates from the "leadership triad" together make up what I call the "leadership excellence" (LE). I recoded the data of MB categories which best described the rectors' perceptions into four categories (A, B, C, and D). Again, I used a combination of $\mathrm{MB}$ scoring guidelines (from the $\mathrm{MB}$ publication). If the leadership score on the relevant items had an average between 1 and 2.5, it meant that the LE was in the "early stage of transition" (A). Scores between 2.6 and 5 suggested that the LE was in the "beginning of systematic approach" (B). Scores between 5.1 and 7.5 suggested a "systematic fact-based process in some part of the organization"(C). Scores between 7.6 and 10 suggested that the leadership approach "is well integrated" (D), and scores close to 9-10 (according to the MB publication) indicate a "fully integrated" leadership triad. Table 8-7 depicts the leadership mean score from the implementation rate Leadership (Category 1) and the last row presents the resulting leadership excellence. 
Table 8-7 Leadership triad and leadership excellence level

\begin{tabular}{lccccccccc}
\hline Leadership & U1 & U2 & U3 & U4 & U5 & U6 & U7 & U8 & U9 \\
\hline $\begin{array}{l}\text { Leadership } \\
\text { Mean score }\end{array}$ & 7.6 & 2.2 & 7.6 & 7.8 & 5.7 & 7.3 & 6.0 & 2.7 & 6.8 \\
\hline $\begin{array}{l}\text { Leadership } \\
\text { excellence }\end{array}$ & C & A & C & C & B & B & C & B & B \\
\hline
\end{tabular}

Two of the nine universities were much lower than the others with regard to leadership (U2, recoded A and $\mathrm{U} 8$, recoded $B$ with an average just above the threshold). Four were in the "systematic fact-based process in some part of the organization" (U5, U6, U8 and U9). Three were just above the threshold of the "well integrated" category (U1, U3, and U4).

\subsubsection{Quality Management Dimensions}

Using the MB survey tool it was possible to fruitfully distinguish several dimensions of Greek universities' practices of partial quality management. I combined different quality management dimensions from the survey and the total overall score that respondents gave. All these measurements provided similar results. In all cases and regarding both the importance and implementation rates, the mean scores were similar, with minor differences. Possibly, this internal consistency hints at good validity and reliability of this measurement.

To summarize the developmental stages in universities, I recoded the data into four categories. Thus, if the university score was between 1 and 2.5 , it was interpreted as being in the "lacking developmental stage". Scores between 2.6 and 5 suggested they were in the "embryonic" stage. Scores between 5.1 and 7.5 suggested they were in the "developing" developmental stage. Finally, scores between 7.6 and 10 suggested that the developmental stage was "advanced". Table 8-8 presents the mean score (implementation rate) for each category of quality management, the total measurement, and the final row presents the recoded level of the quality management practice for each university. Four universities were in the developing stage, one in advanced, three in embryonic, and one in the lacking stage.

Because of the low number of cases, in Table 8-9, I further combined the quality management stages into two categories: lacking and embryonic on the one hand and developing and advanced on the other. Checking against the 'control variables' of university characteristics, no differences were found between the 
quality management practices stage and most of the university characteristics: age, size, range of studies and leadership excellence

Table 8-8 Overall Quality management stages

\begin{tabular}{lccccccccc}
\hline \multicolumn{1}{c}{ QM } & U1 & U2 & U3 & U4 & U5 & U6 & U7 & U8 & U9 \\
\hline $\begin{array}{l}\text { Combined QM } \\
\text { score }\end{array}$ & 6.3 & 2.1 & 7.7 & 5.6 & 4.1 & 4.4 & 7.2 & 4.5 & 6.8 \\
$\begin{array}{l}\text { MB total } \\
\text { overview }\end{array}$ & 6.5 & 2.0 & 7.7 & 6.0 & 4.5 & 4.4 & 7.1 & 4.5 & 6.8 \\
\hline $\begin{array}{l}\text { Developmental } \\
\text { stage }\end{array}$ & $\begin{array}{c}\text { Develop- } \\
\text { ing }\end{array}$ & Lacking & Advanced & $\begin{array}{c}\text { Develop- } \\
\text { ing }\end{array}$ & $\begin{array}{c}\text { Embryo- } \\
\text { nic }\end{array}$ & $\begin{array}{c}\text { Embry- } \\
\text { onic }\end{array}$ & $\begin{array}{c}\text { Develop- } \\
\text { ing }\end{array}$ & $\begin{array}{c}\text { Embryo- } \\
\text { nic }\end{array}$ & $\begin{array}{c}\text { Develop- } \\
\text { ing }\end{array}$ \\
\hline
\end{tabular}

Table 8-9 Quality management stages by university characteristics

\begin{tabular}{lccc}
\hline & $\begin{array}{c}\text { lacking } \\
\text { or embryonic }\end{array}$ & $\begin{array}{c}\text { developing or } \\
\text { advanced }\end{array}$ & Total \\
\hline Age & 4 & 4 & 8 \\
Old & 0 & 1 & 1 \\
New & 1 & 3 & 4 \\
Size & 3 & 1 & 4 \\
Small & 0 & 1 & 1 \\
Medium & & & \\
Large & 2 & 4 & 6 \\
Location & 2 & 1 & 3 \\
Urban & & & \\
Periphery & 2 & 3 & 5 \\
Range of Studies & 2 & 2 & 4 \\
Monothematic & 1 & & \\
Multidisciplinary & 3 & 0 & 3 \\
Leadership Excellence & 0 & 5 & 5 \\
A & 0 & 0 & 0 \\
B & & & \\
C & & & \\
D & & & \\
\hline
\end{tabular}

The only possible relationship in this table (but we have to be careful due to the low number of cases) might be that urban universities tend to be more advanced in quality management than peripheral universities, with four in the developing stage as well as in the advanced stage out of the six. Leadership seems to make a difference: a low level of it correlates with low quality management stage and 
conversely, high leadership effectiveness correlates with high development of the quality management stage.

\subsubsection{Analysis of Gaps}

The MB is also an important self-assessment tool. Therefore, a 'gap' analysis was conducted to compare the differences between importance (pressure) and implementation (practice) responses for each individual university. Table 8-10 presents the overall importance and implementation rate and the difference between each university.

Table 8-10 Average gaps between importance and implementation per university

\begin{tabular}{lccccccccc}
\hline & U1 & U2 & U3 & U4 & U5 & U6 & U7 & U8 & U9 \\
\hline Importance Rate & 8.8 & $7, .3$ & 9.6 & 6.6 & 5.4 & 9.9 & 8.8 & 10 & 9.8 \\
Implementation Rate & 6.5 & 2 & 7.7 & 6 & 4.5 & 4.4 & 7.1 & 4.5 & 6.8 \\
\hline Gap & $\mathbf{2 . 3}$ & $\mathbf{5 . 3}$ & $\mathbf{1 . 9}$ & $\mathbf{0 . 6}$ & $\mathbf{1 . 1}$ & $\mathbf{5 . 5}$ & $\mathbf{1 . 7}$ & $\mathbf{5 . 5}$ & $\mathbf{3 . 0}$ \\
\hline
\end{tabular}

Regarding the gap scores, I distinguished two sizes of gaps: a high range from 5 and up (large gap) and a low from 3 and less (small gap).

Six out of the nine university rectors perceived overall a "small gap"; in other words they found that the implementation of quality management was fairly balanced with the pressures (needs). Small gaps were present mainly in old universities and-by definition-in those with the higher stages of quality management (Table 8-11). In addition, a small gap was noted in one university in the embryonic quality management stage.

Regarding the leadership excellence, small gaps occurred mostly in category C"systematic fact based process in some part of the organization". Large gaps predominated in the A and B categories of leadership excellence. Due to the low number of respondents I did not analyze the gaps per MB categories for each university in detail. ${ }^{27} \mathrm{I}$ observed, though, that on average the smallest gap occurred in Category 6 "Process Management" and the greatest gap was found in Category 2 "Strategic Planning". 27 The overall gap analysis (Table B3 in Appendix) shows the differences between each MB categories
and the responses' importance and implementation scales. 
Table 8-11 Gap overall analysis

\begin{tabular}{lccc}
\hline & Small & Large & Total \\
\hline Age & & & \\
\hline Old & 5 & 3 & 8 \\
New & 1 & 0 & 1 \\
\hline Size & & & \\
\hline Small & 3 & 1 & 4 \\
Medium & 2 & 2 & 4 \\
Large & 1 & 0 & 1 \\
\hline Location & & & \\
\hline Urban & 3 & 2 & 5 \\
Periphery & 3 & 1 & 4 \\
\hline Range of Studies & & & \\
\hline Monothematic & 3 & 2 & 5 \\
Multidisciplinary & 3 & 1 & 4 \\
\hline Leadership Excellence & & \\
\hline A & 0 & 1 & 1 \\
B & 1 & 2 & 3 \\
C & 5 & 0 & 5 \\
D & 0 & 0 & 0 \\
\hline Quality Management Stage & & \\
\hline Lacking & 0 & 1 & 1 \\
Embryonic & 1 & 2 & 3 \\
Developed & 4 & 0 & 4 \\
Advanced & 1 & 0 & 1 \\
\hline & & &
\end{tabular}

\subsection{Summary and Conclusion}

The aim of the survey reported in this chapter was to apply the MB to approach the adoption of quality management through the rectors' and vice-rectors' perspectives. Through this tool, I gathered data about isomorphic pressures, university characteristics, and multiple ideas about the adoption of quality management at the meso level in Greek higher education. Due to the low response rate, I cannot make generalizations, although the results will help provide a better understanding of reasons for (not) adopting quality management.

Overall findings regarding neo-institutional pressures indicated that normative and mimetic pressures were perceived as high in almost all nine cases, and there was almost no inter-university variation between these pressures. Coercive 
pressure was not included in this survey, as the quality assurance law was submitted to the Greek parliament for discussion only in the period when the survey was held (May 2005). In much of the empirical literature, the dichotomy between mimetic and normative pressure is vague. Therefore, studies similar to mine suggested combining normative with mimetic pressure. Nevertheless, I did not follow this pattern, as the MB criteria gave me the flexibility to separate these types of isomorphic pressures (normative and mimetic).

The MB survey showed different views on quality management, bringing the complexity of these practices into focus. For example, all nine universities answered that they did not have any quality assurance system. However, evidence on the implementation stage of actual quality management instruments revealed that four out of nine universities were in a "developing" stage of quality management according to the MB criteria. There is even a possibility that one university (U3) already achieved an advanced stage. Only one university's quality management was regarded as lacking and three universities had embryonic quality management. Maybe quality management was such a debatable concept in this period and so foreign to the leaders that even the universities that implemented quality management procedures did not recognize them as such. The activities were there, but in a disjointed fashion, so that there was not a "system" of quality management in the respondents' eyes.

The study's findings suggested that the implementation of quality management was most advanced in the category "Process Management". In contrast, implementation of Categories 2 "Strategic Planning" and 4 "Measurement Analysis and Knowledge Management" was least developed. Furthermore, analysis of the "gaps" that existed between the desired levels (a consequence of pressure) and the actual implementation of the different aspects of quality management in each university also showed that across the nine universities the smallest gap existed in "Process Management" (Category 6) and the largest in "Strategic Planning" (Category 2). The Category "Process Management" is the focal point within the MB criteria for all key management processes. Blazey et al. (2003) stated that "built into the category are the central requirements for efficient and effective process management-effective education design and delivery, a focus on student learning, prevention orientation, evaluation and continuous improvement, linkage to suppliers, and overall high performance".

One respondent in a personal communication noted that some of the items in category "Process Management" were related to requirements derived from the frame-law 1268/82 that all universities in Greece need to follow. This shows that some coercive pressure existed in the existing regulative arrangements even 
before they explicitly started to address quality assurance, where I was looking for normative pressure. It is possible therefore, that the leaders understand this category better, or were more familiar with this category as a result of it being part of long-standing practices. Moreover, they might see this as a means to gain control over the behavior of the (otherwise very autonomous) professors, rather than allowing their own behavior to be controlled by formalizing strategic planning.

As I stated, the largest gap appeared in "Strategic Planning" (Category 2). This category examines how the organization sets strategic directions and how it determines key plan requirements. It also focuses on how the university evaluates and improves its strategic planning processes. Leaders (rectors and vice-rectors) may use information from Category 2-type activities to set direction and goals, monitor progress, make resource decisions and take corrective action when progress does not proceed according to plan. Blazey et al. (2003) noted that "the key role of strategic planning is to provide a basis for aligning the organization's work process with its strategic directions, thereby ensuring people and process in different parts of the organization are not working at cross-purposes" (p. 107). The authors observed that "to the extent that alignment does not occur, the organization's effectiveness and competitiveness is reduced" (p. 117). The large gap in this category may indicate that universities in Greece were not very aligned, yet they could survive and succeed without this alignment. As mentioned previously (chapter 5), universities in Greece are legal entities under public law, with full self-administration under the supervision of the Ministry of Education, in accordance with article 16 of the Constitution. When the state's laws and supervision prescribe the mission of universities in a single, normative statement, there may be no need and even no possibility for strategic planning.

Furthermore, aspects of the strategic planning category ask the organization to provide a projection of key performance measures and indicators and target goals for both short- and longer-term planning time horizons. Blazey et al. (2003, p. 121) observed that "this projected performance is the basis for comparing past performance and performance relative to competitors and benchmarks, as appropriate". Greek-and many other European-universities achieve their goals in a less competitive environment. As noted earlier (chapter 5) the funding formula is derived primarily from quantitative input factors, without evaluation of their efficiency and in the absence of any concept of varying financial rewards depending on the quality of the offered education and/or the students' socioeconomic background. In addition, the number of new undergraduates accepted to each department of each university every year is determined by the Ministry of Education. In contrast, the universities have autonomy in the selection of 
graduate students, although many graduate studies in Greek universities are offered for free, without tuition fees. The only competition (in terms of funding) one could see in research, but that did not figure prominently in the MB survey. Research and teaching activities, as noted earlier (chapter 5) reflect the principle of academic freedom. In addition, laboratories have full autonomy in the way they organize and conduct research. This implies that there may also be less room for Greek university leaders to develop strategies. However, in the rapidly changing environment, particular pressures for adoption of strategic planning may arise and that may influence future leaders' attention to strategic management.

Analysis of the lacking and embryonic vs. developing and advanced quality management stages revealed that there were no significant correlations between the quality management stage and the university's characteristics age, size, or range of studies. However, urban universities tended to be in the developing stage.

The literature emphasized that a major aspect of quality management is the senior leadership's commitment and active pursuit of continuous improvement. Conyers and Ewy (2004) are among many authors who perceived that "what determines how great an organization will become is a leadership system with clear and measurable organizational values, directions, and expectations. It is not by chance that Category 1 of the Baldrige criteria is focused on leadership". The authors believed that the basic challenge to senior leaders in educational organizations is that there are no excuses for not moving ahead. In order to address the leadership role regarding quality management I developed the "leadership excellence" category. Only two out of nine universities were much lower than the others with regard to leadership excellence. The leadership excellence score was related to quality management practices. Evaluation of quality management practices suggested that not all leaders were fully committed or knowledgeable about quality management initiatives. It is remarkable that the four "laggards" (low scores in leadership) indicated lower implementation of quality management. Not surprisingly, then, overall "small gaps" between pressure and implementation rates occurred only in universities that also achieved the higher the leadership category, "systematic fact-based process in some part of the organization".

Last but not least, I paid special attention to the MB categories with many "no opinion" responses. "No opinion" responses per item and per category were potentially meaningful answers, as these items and categories might indicate the 
extent to which leaders were knowledgeable 28 about them, and perhaps to what extent they perceived these items to be useful to the universities' issues and problems. Category 4 "Measurement Analysis and Knowledge Management" counted most "no-opinion" responses in both importance and implementation categories. Moreover, the same category was rated less important than others by the majority of the Greek university leaders. Therefore the gap between importance and implementation in this category was not so large. Category 4 is directly connected with quality management issues; it is the "brain center" for the alignment of an organization's operations with its strategic directions. Blazey et al. (2003, p. 153) observed that since information and analysis might themselves be sources of competitive advantage and productivity growth, Category 4 also may have strategic value and should be considered as part of the strategic planning process. The facts that Category 4 was rated as the least important by Greek leaders and that it was the category with most "no opinion" answers indicate that these universities have not mastered the process of using data for improvement or for decision-making.

It is likely, as this research took part precisely when quality management was so prominent in the national press (May 2005) that many university leaders did not want to participate in the survey. It is conceivable that they, wrongly, perceived this research as (secretly) organized by the Ministry of Education and did not wish to be regarded as being in league with it. The clear message that I received from these findings was that further research had to wait because the universities were not ready. Therefore, the next empirical study was not administrated until 2007.

The study reported in this chapter was a mail survey, so it was limited by university leaders' willingness to participate in this survey. Learning from this experience, phone interviews or face-to-face interviews were regarded as more appropriate research methods, because they would enable more direct contacts to achieve cooperation. Moreover, it might be possible to attain background information regarding normative, mimetic pressure, and quality management practices by providing beyond these questions. The success of this approach will be shown in chapter 10 .

\footnotetext{
28 A study by Williams and Levy (1992) in Baruch (1993, p. 53) found that self-assessment appraisal was improved when people had accurate knowledge of the performance appraisal system.
} 


\section{The Adoption of Quality Management: Perceptions of Department Heads}

\subsection{Introduction}

The previous empirical chapters already gave some indication of the role of organizational factors for stability or change concerning the adoption of quality management in Greek higher education. One of the core assumptions of this study is that if academics resist or have a different understanding of quality assurance than the policy makers or institutional leadership, quality management will not be effective. Academics' cooperation in quality improvement is therefore crucial. Such cooperation might even be more critical in countries like Greece, where the issues of quality management and quality assurance are quite new phenomena, because quality management and assurance do not easily fit in with the traditional academic culture. While quality assurance is often seen as a policy instrument of governments, quality and excellence are actually 'made' by the 'professionals' on the shop floor, i.e. the academic staff members (Westerheijden, Hulpiau, \& Waeytens, 2007). Department heads form a central link to effective implementation of new practices. Previous research (de Boer, 2003; Ursin, 2007) has shown that department heads' perceptions are critical to the successful adoption of quality management because implementation requires support at the top in the departmental level. Within this background, the raison d'etre of this chapter is to provide insight into the perceptions of the department heads as it relates to the adoption of quality assurance, based on the findings of a survey administered in 2007. As mentioned earlier in this book, Greek university departments are organized around disciplines that form departments, the principal academic units. Heads of department are therefore the micro-level leaders on this study.

\subsubsection{Methodology}

I employed an electronic survey among department heads largely modeled on the questionnaire instrument used by Ursin (2005, 2007). In Finland, Ursin (2007) carried out a survey to find out how quality assurance systems were understood by the academic unit heads. The main goal of Ursin's research was to analyze how Finland's universities experienced the launch of internal quality assurance systems, which also included how the various actors in the adoption process reacted. It seemed appropriate to me to use such a tested instrument to examine 
the adoption of quality management in my study.

\section{Survey Instrument}

A two-part instrument was employed for this study. The first part was a modified version of the instrument developed by Ursin $(2005)^{29}$. Ursin $(2005,2007)$ stated that the aim of his research question was to explore the role of quality assurance and internal quality assurance systems as a part of the departments' quality concerns. The guiding research question in this survey was: How is quality assurance thinking viewed in the basic units? The survey intended to investigate three different aspects of quality assurance:

- How well are quality assurance systems known by department heads?

- How widely quality assurance systems are applied or thought to be applied?

- How useful a tool is a quality assurance system considered to be in promoting the department's basic functions?

In my instrument (see Appendix C), six questions were designed to obtain information regarding the "awareness" category. Another six questions were designed to obtain information on the "applicability" category. And eight questions were designed to obtain information on the "usefulness" category. Five questions were included about the actual development stage of quality assurance systems and related issues. For each of these dimensions, there were both closed questions with five-point Likert-scales and open-ended questions in which the respondents could freely express their views and opinions.

The second part of the survey, which was especially developed for this study, consisted of six additional closed questions with five-point Likert-scales addressing department heads' perceptions of institutional pressures (coercive, normative, and mimetic) towards the adoption of quality management (i.e. quality assurance systems). Respondents' background information was collected as well. Both the survey and the introduction letter asking for participation were in English to stress the comparative aspect (see Papadimitriou et al., 2008) and to create distance from Greek politics in order to stimulate response.

\section{Pilot Study}

The survey tool was piloted with two department heads from one university. After this pilot, no changes were made to the survey instrument, as there were no comments. Both respondents were very satisfied with the questionnaire;

\footnotetext{
${ }^{29}$ Ursin gave permission to use the survey instrument (personal communication, Sept. 2006).
} 
however, they feared a low response rate: "Usually our colleagues don't respond to surveys".

\section{Survey Sample and Response}

The department heads were identified from Greek universities' websites. Between September and December 2007, a full sample of all 266 department heads in Greek public universities received an invitation to participate in the survey. Dillman (2000) and Czaja and Blair (2005) suggested a three-phase followup in order to increase the response rate. I sent out five reminders using both English and Greek e-mail titles and texts. 22 e-mails bounced back and did not transmit. Of the 244 department heads which received the survey, 23 replied with a filled-in questionnaire. The response rate was thus very low $(8 \%)$ and does not allow generalization of the findings.

The breakdown of responding survey participants per university and per discipline is shown in Table 9-1. Data were received from department heads from 11 universities and four disciplines. 16 out of 22 questionnaires were from respondents from universities that participated in the EUA evaluation process. We may assume that representatives from these universities are more familiar with and more open towards quality management issues. Our sample is thus likely to show a positive bias towards quality management issues.

Table 9-1 Survey response by discipline and by university (absolute numbers)

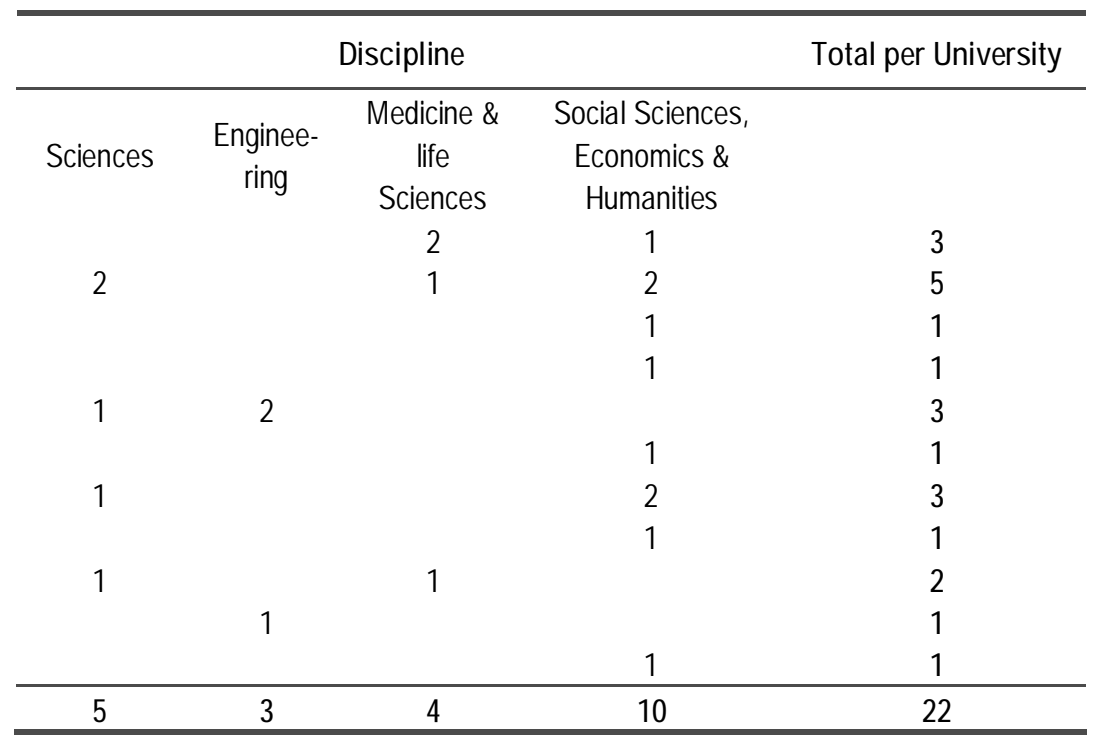




\section{Reliability and Validity}

Content validity of the survey's part one had been previously validated by Ursin (2007). He determined the reliability of the research instrument showing reliability coefficients that ranged from .73 to .91 for his three scales. Part two of the survey was designed and pilot tested for this study. Thus, face validity and content validity were determined using references to neo-institutional literature and the judgment of the participants via the pilot study. Reliability scores could not be established due to the low response.

\section{Data Analysis}

I had planned to organize the participants into eight groups based on their discipline and use variance analysis (ANOVA) to examine the differences between disciplines. This type of analysis needs $\mathrm{N} \geq 30$, so only a basic analysis could be employed.

\subsection{Findings}

\subsubsection{Background Information}

The demographic variables provided additional information about who the survey respondents were. Practically all (21 out of 22) of the Greek departments that participated in the study belonged to a multidisciplinary university. The respondents were apart from one exception professors (21 out of 22). The size of the basic units varied between 13-125 employees. The majority of the respondents had a long working history at the university sector. Only one of them was employed for less than a decade (9 years). 91\% of the respondents (20 out of 22) had never participated in training for quality assurance, while two (9\%) had (missing $\mathrm{n}=2$ ).

\subsubsection{Familiarity, Usefulnes, and Applicability of a Quality Assurance System}

Departments' heads were asked to evaluate their familiarity (awareness) with quality assurance systems as well as the applicability and usefulness of quality assurance systems to departments (Table 9-2). The respondents thought they were familiar to some degree ( 3.7 out of 5 , on a scale from $1=$ fully disagree to $5=$ fully agree with quality assurance systems. The respondents observed quality assurance systems as applicable (4.0 out of 5) and useful (3.9 out of 5) tools to assess academic activities. Participants who had had previous quality assurance training thought that they were less familiar (3.5) with the quality assurance systems than those who had not been trained. However, it seems that 
participation in quality assurance training increased awareness and positive attitudes towards quality assurance. Quality assurance trained participants scored higher in usefulness (4.5) of quality assurance and applicability (4.4).

The respondents' work experience at the university made a difference for familiarity: less experienced department heads ( $<18$ years work experience) felt less familiar (3.7) with quality assurance than those with very long ( $>25$ years) experience (4.1). Department heads with less than 18 years work experience thought that quality assurance was more useful (4.3) and applicable (4.5) than the older department heads (> 25 years) (usefulness: 4.0 and applicability: 4.0). In all three respects, the middle group (18-25 years experience) indicated the lowest averages (familiarity: 3.3, usefulness: 3.5 , and applicability: 3.6).

The size of the respondent's department made a difference only with regard to familiarity. Department heads working in large departments ( $>50$ employees) thought that they were more familiar (4.0) with such systems. They thought slightly more often that quality assurance systems were useful (4.3) and applicable (4.3) than department heads working in small and medium sized departments $(3.6)^{30}$.

Table 9-2 Familiarity, usefulness, and applicability of quality assurance systems (means)

\begin{tabular}{lccc}
\hline \multicolumn{1}{c}{$\begin{array}{c}\text { Participants } \\
\text { characteristics }\end{array}$} & $\begin{array}{c}\text { Familiarityl } \\
\text { Awareness }\end{array}$ & Usefulness & Applicability \\
\hline $\begin{array}{l}\text { Participation in QA training } \\
\text { No }(n=18)\end{array}$ & mean & mean & mean \\
Yes $(n=2) \quad$ Missing $=2$ & 3.7 & 3.9 & 4.0 \\
\multicolumn{1}{c}{ Work experience } & 3.5 & 4.5 & 4.4 \\
$<$ 18 years $(n=8)$ & & & \\
18-25years $(n=5)$ & 3.7 & 4.3 & 4.5 \\
$>25$ years $(n=9)$ & 3.3 & 3.5 & 3.6 \\
Department size & 4.1 & 4.0 & 4.0 \\
$<25$ employees $(n=7)$ & 3.6 & 3.9 & 4.1 \\
25-50 employees $(n=8)$ & 3.6 & 4.0 & 4.0 \\
$>$ 50 employee $(n=5)$ & 4.0 & 4.3 & 4.3 \\
$\quad$ Missing=2 & & & \\
\hline
\end{tabular}
${ }^{30}$ With only one respondent from a monothematic university, I could not test the relation between
type of university and views on familiarity, usefulness, and applicability. 
There were some differences between disciplines (see Table 9-3). Respondents from engineering units thought that they were more familiar (3.9 out of 5) with quality assurance systems than department heads from other disciplines; they regarded such systems, however, as less useful (3.6 out of 5) and applicable (3.4 out of 5) in their units. The respondents from medicine and life sciences regarded quality assurance systems somewhat more useful (4.3 out of 5) and applicable (4.4 out of 5) than those from social sciences, economics \& humanities.

Table 9-3 Familiarity, usefulness, and applicability of a quality assurance system by discipline (means)

\begin{tabular}{lccc}
\hline \multicolumn{1}{c}{$\begin{array}{c}\text { Disciplines } \\
(\mathrm{n}=22)\end{array}$} & $\begin{array}{c}\text { Familiarityl } \\
\text { Awareness }\end{array}$ & Usefulness & Applicability \\
\hline $\begin{array}{l}\text { Sciences } \\
(n=5)\end{array}$ & 3.8 & 4.4 & 4.2 \\
$\begin{array}{l}\text { Engineering } \\
(n=3)\end{array}$ & 3.9 & 3.6 & 3.4 \\
$\begin{array}{l}\text { Medicine and Life Sciences } \\
(n=4)\end{array}$ & 3.8 & 4.3 & 4.4 \\
$\begin{array}{l}\text { Social Sciences, Economics } \\
\text { \& Humanities (n=10) }\end{array}$ & 3.7 & 4.0 & 4.3 \\
\hline
\end{tabular}

\subsubsection{Advantages and Disadvantages of a Quality Assurance System}

Of the 22 respondents, 13 perceived quality assurance systems as a benefit or advantage, two did not, and five did not know (missing n=2). From the thirteen department heads who considered quality assurance system as a benefit, seven gave more details regarding how quality assurance could be beneficial. Two responses had to do with the assessment and further development of academic practices; further, one each covered goals, transparency (and competition), and discovering weaknesses. In one answer a special emphasis was on student learning outcomes ('to efficiently produce consistently high quality dentists"). Finally, the opinion of one department head was that quality assurance enabled the university to "establish a culture" (of quality or evaluation).

In their responses to the contrary question, namely if a quality assurance system would hamper the department or be a disadvantage, 11 respondents did not see quality assurance as hampering the operation of their unit, $3 \mathrm{did}, 4 \mathrm{did}$ not know (missing $n=4$ ). The disadvantages mentioned by department heads in the open question were: 
- "The Greek academic environment is not very suitable due to the power of the student bodies and because of their attachment to political parties" (mentioned once);

- "There is a significant amount of work involved in the initial implementation of a quality assurance system" (mentioned once).

Two department heads did not see a quality assurance system as either benefiting or hampering their department, while another two simply were undecided. Finally, two said that quality assurance was both benefiting and hampering their department (to both questions they said yes).

Concerning the correlation between "benefit" of quality assurance systems and usefulness, one may expect to see correlated answers. However, the actual responses were mixed. One department head who reported a quality assurance system as not being of benefit for the department responded that the usefulness of a quality assurance system was 3.8 (i.e. fairly high). Additionally, the mean score of usefulness from the five department heads who reported not knowing whether a quality assurance system was or was not a benefit ranged the usefulness between 3.3 to 3.9. The mean score in the usefulness category for the 13 respondents who perceived quality assurance system as a distinct benefit for their department ranged from 3.5 to 4.9 (i.e. from similar to maybe a little higher than others, the average being 4.0). Looking at these answers perhaps one could say that the expected correlation was not or only very weakly found among this small sample.

\subsubsection{Various Definitions of 'Quality'}

Five department heads provided definitions of how they understood quality assurance. One focused on continuous improvement. Two understood it in terms of consistency (with requirements). Two others dealt with aspects of performance (like showing research output). Another five department heads provided other comments (missing $\mathrm{n}=11$ ).

\subsubsection{State of Development of Quality Assurance Systems}

Another question addressed the developmental stage of quality assurance systems within departments in Greek higher education. The data from the 22 responses to this question indicated that four department heads thought that their department did not have any quality assurance system, nine department heads thought that such systems were in their embryonic stages, eight thought that they 
were in the developing stage, and one department head was not aware of the stage of development (did not know). Additionally, a further question addressed the developmental stage of quality assurance systems within their universities as a whole, where no more than two respondents from the same university reported the same stage for their university's quality assurance system state of development. In fact, of the five department heads from the same university, two reported that their university's quality assurance was nonexistent, one respondent perceived it to be embryonic, and the other two thought it was in the developing stage. Whether this reflected different perceptions of the university's reality, or different understandings of the terms used in the questionnaire, could not be established.

Overall, 17 out of the 22 respondents gave, however, the same rating to both their department and their university as a whole in terms of the development level of their quality assurance systems. The large majority, therefore, did not see a difference between their own department's stage of development and the university as a whole.

Of the five department heads who reported that their university quality assurance system was nonexistent, four were from universities that had already participated in the EUA-IEP. These results seem to indicate that the EUA-IEP was not universally regarded as a process that helped to build a sustainable quality assurance system in these universities.

I draw special attention (Table 9-4) to the data that three department heads who reported the state of development in their departments' quality assurance systems were in the 'lacking' stage, scored between 4.0 and 4.5 in the applicability category. It may look antithetical that quality management would be highly applicable to higher education (denoted by the 4 to 4.5 score), yet it was not applied to their own departments ('lacking' stage). In another, similar antithesis, seven department heads who reported that their quality assurance system was in the embryonic stage scored very high (between 4.2 and 5) regarding applicability of quality assurance. In cases where department heads reported an applicability mean score that high, one would expect that their quality assurance system would in the developing or advanced stage. The other eleven department heads, i.e. about half the respondents, gave answers with corresponding values for the applicability of quality assurance and the current quality assurance stage of their department. How can we explain the antitheses for the first half of the respondents? Possibly, they gave a socially desirable answer on the applicability question. An additional possibility was that even if they genuinely believed that quality management would be applicable to higher education institutions, they 
had not been able to implement it in their own department due to organizational problems (e.g. lack of support among their department's staff, or from the university leadership).

Table 9-4 Familiarity, usefulness, and applicability judgments by stage of development of Quality Assurance (means)

\begin{tabular}{ccccc}
\hline $\begin{array}{c}\text { QA mean score } \\
\text { in 3 categories }\end{array}$ & Lacking & $\begin{array}{c}\text { QA Stage } \\
\text { Embryonic }\end{array}$ & Developing & Don't know \\
\hline $\begin{array}{c}\text { Familiarity } \\
<3.9\end{array}$ & 2 & 1 & 1 & 1 \\
$>4$ & 2 & 8 & 7 & - \\
Usefulness & & & & \\
$<3.9$ & 2 & 3 & 4 & 1 \\
$>4$ & 2 & 6 & 4 & - \\
Applicability & & & & \\
$<3.9$ & 1 & 2 & 3 & - \\
$>4$ & 3 & 7 & 5 & 1 \\
\hline
\end{tabular}

\subsection{Pressures for the Adoption of Quality Assurance Systems}

This research took place during the period September 2007 to December 2007 when the new quality assurance law in Greece had been "formally enacted". We were interested in understanding the pressure that department heads perceived to adopt quality assurance systems. All items in the second part of the survey were designed to determine to what extent department heads might perceive coercive, normative, or mimetic pressures by asking respondents to indicate how many years might be needed until their departments would adopt quality management systems, if at all. The pressure that they felt to adopt quality management systems would be characterized as high if the answer was "in one year". When the answer was "in two years" the degree of pressure would be characterized as medium. Low pressure was assumed with an answer "in three years". A "never" answer indicate no pressure felt. Table 9-5 provides the outcomes of this part of the questionnaire.

\section{Coercive Pressure}

Two questions in the survey instrument addressed coercive pressure. The first question asked if department heads would adopt quality assurance systems in the next 1, 2, 3 years (or never) if quality assurance was required by law. The data from the 21 responses to this question indicate that the largest number, 11 department heads, would adopt quality assurance systems in three years, five 
would adopt quality assurance system in one year, another three would adopt such systems in two years, and two department heads responded that they would never adopt such a system (missing $\mathrm{n}=1$ ).

Table 9-5 Perceptions of isomorphic pressures towards the adoption of quality management (absolute numbers)

\begin{tabular}{|c|c|c|c|c|c|c|}
\hline \multirow{2}{*}{$\begin{array}{l}\text { Isomorphic } \\
\text { pressures }\end{array}$} & \multicolumn{5}{|c|}{ Years required for adoption } & \multirow{2}{*}{ Total } \\
\hline & 1 & 2 & 3 & More & Never & \\
\hline \multicolumn{7}{|l|}{ Coercive: } \\
\hline QA Law & 5 & 3 & 11 & - & 2 & 21 \\
\hline Funding & 5 & 4 & 11 & - & 2 & 22 \\
\hline \multicolumn{7}{|l|}{ Normative: } \\
\hline Employers & 3 & 2 & 5 & - & 8 & 18 \\
\hline Students \& Family & 4 & 1 & 7 & 1 & 6 & 19 \\
\hline Faculty & 7 & 2 & 8 & 1 & 2 & 20 \\
\hline \multicolumn{7}{|l|}{ Mimetic: } \\
\hline $75 \%$ of dept. & 8 & 4 & 8 & - & - & 20 \\
\hline
\end{tabular}

The second question asked if department heads would adopt quality assurance systems in the next 1, 2, 3 years (or never) if quality assurance was required by governmental funding policies. The data from the 22 responses to these questions indicated that the largest number, 11 department heads would adopt quality assurance system in 3 years, five in one year, four in two years, and two respondents would never adopt a quality assurance system.

From these data, I conclude that a majority of respondents in this survey do not think that coercive pressure deriving from legal or financial frameworks would be a force leading to a fast implementation track for quality assurance systems.

\section{Normative Pressure}

Three questions in the survey instrument addressed normative pressure. The first question asked if department heads would adopt quality assurance systems in the next 1, 2, 3 years or never as quality assurance was requested from employers of their graduates. The second question asked if department heads would adopt this system if requested from students and parents, and the third one asked if they would adopt quality assurance systems if these were requested from departmental faculty members.

It might be questioned if these sources of pressure all are associated with professionalization, the reason for normative isomorphism. In other countries 
where students (and parents) pay tuition, perhaps the pressure from students (and parents) is associated with coercive pressure; however, in Greece, undergraduate studies are free so this question was interpreted as normative pressure.

The responses to the first question indicate that eight department heads would never adopt quality assurance systems if requested from employers of their graduates. Five respondents would adopt quality assurance systems in 3 years, three would adopt this system in 1 year, and the smallest number of two respondents in 2 years (missing $n=4$ ).

The responses to the second question indicate that the largest number, seven department heads, would adopt quality assurance systems in 3 years in response to pressures from students and parents. Six would never adopt quality assurance systems. Four would adopt such systems in 1 year, one respondent in 2 years. Another department head commented that he/she would adopt quality assurance systems in more than 3 years (missing $n=3$ ).

The responses to the third question indicate that the largest number, eight department heads, would adopt quality assurance systems in 3 years in response to pressures from their faculty members. Seven would adopt this system in 1 year, two in 2 years, and two would never adopt quality assurance systems. One department head commented that he/she would adopt this system in more than 3 years (missing $\mathrm{n}=2$ ).

From these data I conclude that the majority of the department heads do not think that normative pressures from employers, students or parents would be a strong force leading to an implementation of quality assurance systems at all. In contrast, a majority of the department heads think that pressures from faculty members within the department would be a force leading to an implementation of quality assurance systems even though not necessarily in the "short run".

\section{Mimetic Pressure}

One question in the survey instrument addressed mimetic pressure: "If in your area $75 \%$ of the departments implemented a quality assurance system, do you think that you would implement a quality assurance system in your department in the next one, two, three years or never?" The data from the 20 department heads responding to this question indicated that eight department heads would adopt quality assurance systems in 1 year. Another eight would adopt quality assurance systems in 3 years and four in 2 years (missing $n=2$ ). 
From these data I conclude that mimetic pressures from the organizational environment are perceived as a force towards the implementation of quality assurance systems even though not necessarily in the short run.

Overall, empirical evidence thus indicates that department heads do not think that isomorphic pressures would play a strong role towards the adoption of quality management systems in their department. Mimetic pressure (copying other faculties/departments) is the dominant pressure while normative pressure from the faculty within the department is also perceived as leading to the adoption of quality assurance systems. Coercive pressures from legal or financial frameworks are expected to be a weak force and normative pressures from employers, parents and students are expected to be an even weaker force with respect to the adoption of quality assurance systems.

This overall picture is also confirmed when zooming into the perceptions of those department heads who responded to all questions related to isomorphic pressure $^{31}$ :

1. Disciplinary patterns did not appear, perhaps due to the low total number of responses. Still, it is interesting to realize-as shown by additional analysis that is not easily summarized in a single tablethat one engineering and one economic department (known to have a high prestige) feel impervious even to coercive pressure.

2. Out of the normative pressures, staff pressure is seen as the most powerful. Two department heads (the same ones as in 1) from engineering and economic disciplines, however, did not change opinion and reported that they would never adopt this system, not even if it was requested by their faculty.

\subsection{Summary and Conclusion}

The aim of this study was to provide insight into the perceptions of department heads on the adoption of quality assurance systems. A survey was undertaken among a full sample of department heads in Greece addressing issues of familiarity, usefulness and applicability of quality assurance systems, the state of their implementation as well as addressing department heads' perceptions of pressures towards the adoption of quality assurance systems. Only 22 department heads participated in this survey. The response rate was thus very low (8\%), and does not allow generalizations of the findings. Many respondents in our sample were department heads at universities that had previously participated in a

\footnotetext{
${ }^{31}$ The non-responses do not change the pattern.
} 
European quality assurance exercise, and it is likely that our sample shows a positive bias of respondents towards quality assurance. The survey results reflect nevertheless quite ambivalent perceptions of quality assurance systems, most departments not having implemented quality assurance systems or being in very early stages of adoption, and I noted an overall perception among department heads that isomorphic pressures would not necessarily lead to their adoption, at least not on a fast track.

Data were collected from 11 universities and reflected five disciplines: Science, Engineering, Medicine and Life Science, Social Sciences and Economics, and Humanities. When examining the respondents' views on familiarity, usefulness and applicability of quality assurance systems most of them thought they were familiar with such systems to some extent. The average value for applicability was, however, highest followed by the perception of usefulness while familiarity scored lowest. These perceptions did not differ between respondents who did and those who did not receive formal training in quality assurance. In a comparable survey undertaken in Finland, these relations were different: The average value for familiarity was higher than the one for usefulness, which in turn was still higher than the average values for applicability (Ursin, 2007). Work experience of respondents seems to make a difference only in regards to familiarity. Department heads with less work experience felt less familiar with quality assurance systems. The department heads from the largest departments (more than 50 staff members) reported higher familiarity, usefulness, and applicability than others. Department heads from engineering thought that they were more familiar with quality assurance systems; however, they regarded them as less useful and applicable.

With regard to the adoption of quality assurance systems at the departmental level respondents indicated that they were in most cases either nonexistent or in an early stage of development. Department heads were often not of the same opinion when it comes to the stage of quality assurance systems in their university as a whole. No more than two department heads from the same university reported the same stage for their university's quality assurance system. Where necessary I calculated averages of divergent opinions to arrive at an overall impression of the state of development of quality assurance practices of the universities. It then appears that ten quality assurance systems were seen to be in the developing stage, six in the embryonic stage, five lacked a quality assurance system, and one department head did not know.

Among our respondents, there seemed to be an apparent uncertainty about the role and effects of quality assurance systems in their units, and that uncertainty 
engendered a more reticent behavioral attitude towards adoption than positive attitudes. Some respondents did not have a clear view on what quality assurance or quality was, but they did have high expectations of it. The majority view regarding the advantages of quality assurance in departments were related to evaluation and development activities of the primary processes. Disadvantages were mentioned by only few respondents. One department head who perceived the quality assurance system as a disadvantage mentioned the role of the academic environment: "The Greek academic environment is not very suitable due to the power of the student bodies and because of their attachment to political parties". Another one from the same category noted that "there is a significant amount of work involvement in the initial implementation of a quality assurance system". Another one who did not know if quality assurance was a disadvantage wrote: "Many times new ideas need time to be implemented and therefore the expected benefit will come in the long run".

In terms of isomorphic pressures at the departmental level, department heads mostly thought that mimetic pressures (i.e. many other departments already adopted quality assurance systems) would lead them to adopt a quality assurance system. Normative pressures (from faculty within the department) were also considered as leading to adoption even though not necessarily on a fast track. Coercive pressures (from legal and financial frameworks) as well as other normative pressures (from employers, family and students) were perceived as weak forces when it comes to the adoption of quality assurance systems. In the international quality assurance literature "outside" stakeholders such as employers, alumni, and family were often mentioned as drivers for adoption of quality assurance, but in Greece their influence would seem to be remarkably low. The strength of mimetic pressure perhaps was the most "unexpected" finding in this study.

I conclude this part with one department head's remark: "The Greek academic environment is a very difficult one for implementation of a quality assurance system. The legal framework is present but in my opinion it shall never be implemented. No one really wants it and if ever implemented it shall be only paper". For the moment, the adoption of quality assurance at the departmental level in Greek higher education is only in its infancy. While early steps have been taken, full understanding of the role of quality assurance for higher education is lacking. Until 2008, less than 30 out of 266 departments decided to adopt quality assurance systems indicating that this is a major challenge in Greek higher education. The future implementation of quality assurance systems in departments as a mandatory governmental tool might eventually produce different outcomes. After three or four years of operation, the new Hellenic 
Quality Assurance Agency will have to publish departmental evaluation reports. Therefore, after 2011, a closer look will be possible at if and how departments will have adopted quality assurance systems-at least formally-and if there will be more differentiation among them. Then this study could be revisited for further research. 


\section{The Use of ISO Standards in Greek Higher Education 32}

\subsection{Introduction}

ISO standards provide a framework for quality management in organizations. The challenge to any organization is to invest in the monitoring of quality in such a way as to improve profitability and effectiveness. The brochure "ISO 9000 Selection and use, 1998" states that "the ISO family of international quality management standards and guidelines has earned a global reputation as the basis for establishing quality management systems" (p. 1). It is suggested that, beginning with ISO 9000:2000, organizations adopt ISO 9001:2000 to achieve a first level of performance.

Although the ISO 9000-series quality management system was designed for manufacturing, ISO standards have also become a popular choice for educational systems worldwide (Bevans-Gonzales \& Nair, 2004; Karapetrovic, 2002; Peters, 1999; Singh \& Sareen, 2006; Thonhauser, 2005; Zuckerman \& Rhodes, 2000). The literature surrounding ISO implementation in education, as noted earlier (chapter 2 ), indicates that the application of this quality management system to education remains debatable, arguing that the implementation process is time-consuming and difficult, and the subject is understudied empirically (Lundqvist, 1997; Thonhauser \& Passmore, 2006). The scope of this study was not intended to end the debate, but rather, to contribute an empirical study that might improve our understanding of how ISO standards are presented, regarded, and applied in Greek universities.

Taking into consideration that in 2006, the quality assurance system in Greece was in a very early and debated stage; therefore, the intention of this final empirical study is to present the extent of use of ISO standards in Greek universities up to 2006, and simultaneously to examine isomorphic pressures that might influence the adoption of this particular quality management practice.

\footnotetext{
32 This is a revised version of the article: Papadimitriou, A., and Westerheijden, D.F., (2010) “Adoption of ISO-oriented quality management tools in Greek universities: Reactions to isomorphic pressures," TQM Journal 22 $\beta$, pp. 229-241.
} 


\subsection{Methodology}

This study employed sequential qualitative data collection by using a survey and telephone interviews. The study involved two phases. Each phase was designed with a specific purpose in order to meet the needs of the overall study. Two sets of data were mixed between the two phases, while selecting the participants for further interviews for the follow-up analysis based on the results of the first phase (Creswell et al., 2003). The first phase was designed to answer questions such as: Can I find ISO standards, as quality management practices, at the micro level in Greek higher education? And if so, how many units are implementing ISO standards? What types of ISO standards can I find? Which types of schools, academic departments, and laboratories are implementing or are in the process of implementing ISO standards? And finally, can I find ISO in academic support services, and if so, where? The second phase focused on isomorphic pressures that directors (departments' heads, laboratories' and academic support services directors) felt in order to adopt ISO standards in their domain (if any).

\subsection{First Phase: Finding Out the Extent of Use of ISO Standards in Greek Higher Education}

Research at this particular micro level in Greek higher education, was conducted over several months (September 2005-April 2006) and in several ways. It was challenging to identify units in Greek higher education that had ISO standards. Other researchers have faced a similar struggle and in order to find institutions for his research, Lindqvist (1997) used several methods. He wrote: "the search for published material of relevance resulted in some hints, but the primary sources for information were informal channels such as so-called list-servers, on the internet, university web-pages, personal contacts, and contacts with several national and International Organizations for Standardization" (p. 135). Similarly, Thonahauser (2005, p. 23) noted: "the issue of population of these educational institutions proved to be a large and complicated endeavour". Despite the fact that ISO functions in a highly rationalized and technical environment, surprisingly, Thonahauser found the central body of ISO and the national bodies to be poorly organized and vague (perhaps by choice) in respect to maintaining records of ISO 9000 registered institutions. This experience matches the description of the standardization sector by Loya and Boli (1999) as "almost incomprehensibly complex... even many participants have fuzzy and incomplete images of their place in the whole". This description becomes even more evident as I attempted to identify ISO 9000 registered educational institutions for this research. Thonahauser (2005, p. 30) aptly described her efforts to verify her population: "all of the organizations mentioned were unable to verify or provide 
more information regarding the number of ISO 9000 registered educational institutions. Most of the responses were as follows, 'I could find no resource for the schools registered to ISO 9000 in Great Britain' (Department for Education and skills [DfES], personal communication, October 21, 2004)". I faced similar circumstances conducting this survey.

The ISO (the central body) does not keep records of the number of registered organizations. I had e-mail communication with ISO in Geneva, Switzerland, that advised me to contact the Greek Organization for Standardization (ELOT ${ }^{33}$ ). Furthermore, there were several national member bodies and independent ISO registrars operating therein. Educational institutions or laboratories interested in registering to ISO 9000 needed to apply via the ISO local bodies. ELOT provided me with some information; however, its webpage provided data only through 2005. Therefore, further data were collected from independent ISO 9000 registrars. Accordingly, I had direct communication with the Hellenic Accreditation system (ESYD) 34 and I collected data from its webpage. Communication with the Greek organizations was similar to Thonahauser's (2005) experience: registrars' data bases were scanty. In the end, the data collected for this study were derived mostly from the Greek universities' and laboratories' websites.

\subsubsection{Findings}

ISO standards were applied in seven out of 21 Greek universities. In these universities, ISO 9001:2000 and other quality management systems, such as ISO 17025 , could be found at the micro level. ISO/EC 17025:2005 defined general

${ }^{33}$ According to law 372/76, ELOT (Hellenic Organization for Standardization) is a national member and participates in the following international standardization organizations: International Organization for Standardization (ISO) and International Electro-technical Commission (IEC). Public service organizations, educational institutions, and industrial sectors can follow or participate through ELOT in the work of international standardization on the subject in which they are interested. They can be informed and express their opinions on the content of international standards. They can also participate and support the Hellenic interests at the relevant Technical Committees of the mentioned International Organizations.

34 The Hellenic Accreditation System S.A. (ESYD) was established in 2002 and succeeded the Hellenic Accreditation Council, which under the same distinctive title, had operated within the Ministry of Development after 1994. ESYD is a private liability company operating in favour of the public interest with the responsibility of the management of the accreditation system in Greece. The share capital of the company has been undertaken by the Greek State. It is foreseen, however, that shares up to $40 \%$ are transferable to legal entities of the public or of the private sector, with scope of activities related to quality issues. Within the Organs of the Company (Managing Board and Hellenic Accreditation Council), a well-balanced representation of Ministries, scientific associations and professional and social unions is provided, safeguarding independence and impartiality in its activities. 
requirements for testing competence and the calibration of laboratories. It specified the general requirements for the competence of staff to carry out tests and/or calibrations. There were 15 management requirements and 10 technical requirements that spelled out what a laboratory must do to become certified.

If a university was implementing ISO standards, it was usually in a separate laboratory or academic support service, but not in the entire university. Interestingly, I found one university implementing the EU Eco-Management and Audit Scheme (EMAS) ${ }^{35}$ throughout the whole institution. Out of the wide variety of laboratories implementing ISO standards, ISO 9001:2000 and ISO 17025 were most popular in engineering (mechanical, chemical, and civic) laboratories, and in laboratories in the medical field (4 cases). On the other hand, academic support services such as research committees and libraries had implemented ISO 9000, too. One library followed the procedures and regulations, prescribed by ISO, and another was in the process of registering. The first ISO standards appeared in 1994, and several laboratories and the research committee registered several times since.

Table 10-1 presents the use of ISO standards in Greek higher education according to a search of university websites. The first column specifies types of ISO standards within Greek universities ${ }^{36}$. The other columns show the number of laboratories and academic support services with ISO standards as appeared on their website-these laboratories and academic support services belong to the university coded $\mathrm{Ua}, \mathrm{Ub}$, etc., in the first row.

Table 10-1 Use of ISO standards in Greek higher education

\begin{tabular}{ccccccccc}
\hline ISO Type & Ua & Ub & Uc & Ud & Ue & Uf & Ug & ISO cases \\
\hline $\begin{array}{c}\text { ISO 9001:2000 } \\
\text { EN45001/ }\end{array}$ & 12 & 3 & 3 & 2 & 1 & 1 & & 22 \\
$\begin{array}{c}\text { ISO 17025 } \\
\text { EMAS }\end{array}$ & 4 & 2 & 2 & 1 & & & & 9 \\
\hline $\begin{array}{c}\text { 1st phase } \\
\text { Total cases }\end{array}$ & 16 & 5 & 5 & 4 & 1 & 1 & 1 & 32 \\
\hline
\end{tabular}

35 http://ec.europa.eu/environment/emas/index_en.htm.

36 University type in this particular study does not follow the categorization presented in previous studies in order to keep the anonymity of the university. 
As stated earlier, finding data from Greek organizations has been problematic. University web pages sometimes proved to contain incomplete, inaccurate, or expired information. In some cases, university laboratories' information was contained at the (micro) department/laboratory level and the importance at the (meso) university level was not emphasized. In my study, one of the methods I employed to adjust for this error during the second phase was to rely upon telephone interviews using direct questions to determine whether ISO standards were being used. As a result of the phone interviews, which will be discussed next, I had to adjust the initial findings of 35 cases down to the 32 cases shown in the table, where ISO standards were actually in use.

\subsection{Second Phase: Isomorphic Pressures and ISO Standards}

The second phase focused on isomorphic pressures and the adoption of quality management systems such as ISO 9001, ISO 17025 or EMAS. For the purpose of this phase, I had to select the most appropriate individuals to answer the questionnaire. Thus, I followed Kraut's (1996) suggestions for organizational surveys. The executives (directors) of each unit were the most appropriate individuals who were able to offer detailed information about the adoption of ISO standards. In a similar vein for her study, Thonahauser (2005) had chosen specific individuals to answer her questionnaire and not the entire institution. In her case, the individuals most involved in the ISO 9000 activities were either the director or the principal of the institution, or a different member of the staff who had been assigned to oversee the ISO 9000 implementation process.

I used contact information (director's name and telephone number) obtained from the first phase. During phone calls I discovered that some of the databases provided by the registrars and publishing companies were not accurate or up-to date. Three units were no longer using ISO standards. Similar issues were addressed in Thonahauser's (2005, p. 31) study as well.

\subsubsection{Survey Instrument}

The survey questions were written according to the suggestions and guidelines provided by Kraut (1996), Fowler (1993), and Dillman (2000). I also took into consideration suggestions from Thonahauser's (2005, p. 32) study. The content of the interview's open-ended questions (see Appendix D) was based on the theoretical part of this study extracted from the literature on quality management and isomorphism (coercive, normative, and mimetic). 
The first part of the questionnaire requested participants to verify if the information obtained through the webpage was accurate. These items in the questionnaire were designed to obtain details such as the type of the ISO standards, the status of whether this situation is in process or not, how many times they registered before, and the interviewee's profession. These questions at the same time served as ice-breakers (Hatch, 2000). Another aspect of the interview was the contact I made with the participant and the explanation I provided for the purpose of this survey. In the beginning, I introduced myself and explained that this was the second phase of my study; thus, I would like to know why they adopted this quality standard, for what purpose, and if there was any pressure or motivation to adopt certain standards

Next, questions were asked about neo-institutional pressures: coercive, normative, and mimetic. Mizruchi and Fein (1999) discussed how it was difficult to distinguish strands of isomorphism in practice, yet my questionnaire was designed to make precisely those distinctions. In regards to coercive pressure, I used questions that asked about particular pressures from existing laws and regulations; I also asked if they experienced any pressures regarding funding through projects, with principals pressing them to adopt this quality management practice. As regards mimetic pressure, I posed questions about copying or mimicry of other similar units. In order to find out if there was any normative pressure, I asked questions about professional networks, director's involvement in ISO adoption, and decision-makers demonstrated commitment to ISO standards. Questions such as the costs of ISO standards and its benefits also are appropriate to determine the type of pressure. In other words, I guided participants in a Socratic way to achieve deeper understanding of the types of isomorphic pressures.

The final questions were designed to define consequences of the adoption of ISO standards, whether they were regarded as beneficial or negative to the university as a whole and in what ways was it connected to the quality of the university. I also added a number of probing questions to each open-ended question to ensure all aspects of the complex phenomenon were discussed during the interview. The last question asked participants for any additional information they might add in regards to pressure and the adoption of ISO standard as a quality management practice.

The interview protocol was pilot-tested on two participants randomly selected from those who responded in the first quantitative phase of the study. Based on the pilot interview analysis, the protocol was not revised, as there were no comments. Additionally, the results of this pilot study showed that the questions 
were understood well by the directors, and they expressed their willingness to participate in this interview. The results of these two pilot tests were surprisingly positive. Both directors were open to participate in this type of research and added additional information at the end of the interview regarding the consequences of ISO practices and some informal thoughts regarding quality assurance systems and higher education in general.

\subsubsection{Population Characteristics}

Phone calls allowed me to verify data and my list of laboratories (first phase). I discovered that my original lists from publishers and ISO registrars were neither accurate nor up-to-date. Therefore, the population of this study was 32 ISO-using cases that I contacted. Two respondents refused to participate in a phone interview. Seven directors could not be reached. Thus, evidence for this part of analysis was derived from a total of 23 interviews (response rate $72 \%$ ).

The frame-law in Greek higher education states that only a professor can be elected as a director in a university laboratory (see Section 5.1.3.2); thus, the majority of the interviewees were professors. In two cases, I interviewed ISO representatives following the directors' desires. Three academic support services directors were also administrators. In total, four were women and 19 men. The interviews took place during November and December 2007. Every interview required approximately 10 to 15 minutes and I kept handwritten notes. Utilizing this procedure in data collection allowed me to ask clarifying questions on issues concerning ISO practices and to investigate isomorphic pressures.

Organization of data is critical in qualitative research; therefore, I developed a matrix to organize the interviews. As the amount of data was small (less than 500 pages) I used hand analysis. I analyzed the data by following Creswell's (2003) and Miles and Huberman's (1994) suggestions to develop a deeper understanding about information supplied by my participants.

\subsubsection{Findings}

This section addresses the issues of quality management practices such as ISO standards by asking about enabling pressures for adoption. Based on the theoretical framework adopted for this study, the test for isomorphic pressures involved a study of three types of pressure: coercive, normative, and mimetic. If these types could be identified within the interviews, an isomorphic pressure was assumed to be present. If these types were not detected, a neo-institutional 
pressure was deemed not to exist in the particular ISO case. Table 10-2 summarizes the outcomes of this study.

The following statements were typical examples of isomorphic pressures:

Coercive: "ISO certification was requested by a funding project or stakeholders needs".

Normative: "I come from abroad and have experience with these applications," or "our laboratory belongs in a network", or "ISO adoption was a collective decision".

Mimetic: "It was not necessary for the moment, perhaps in the future", "we don't know ISO benefits yet"; or "was an opportunity for our university" (all statements implied an unspecified, uninformed feeling of low pressure), "ISO is associated with prestige to the whole university", or "similar units [laboratories or/and academic support services] abroad use ISO a lot for their services" and "our personnel adopted ISO by themselves according to the consultants' advice".

The qualitative analysis of the data suggested that in laboratories that employed ISO 9001:2000, the type of pressure observed was a combination of coercive and normative pressures in the majority of the cases (7 out of 13). Coercive pressure on its own was detected in three laboratories holding ISO 9001:2000. Only in two laboratories, holding ISO 9001:2000; normative pressure on its own appeared in their directors' interviews. In all three academic support services, the pressures perceived were normative and mimetic. Moreover, these interviewees commented that they did not copy any similar services in Greece. These comments were understood as reflecting mimetic pressure such as "an attempt to model best practices". The following examples support the fact that there was mimetic pressure:

- The Research Committee's ISO practice is observed as the only case within Greek universities. It wanted to solve problems in its services and therefore adopted ISO as happens in the private sector.

- In both library cases, the respondent highlighted that she/he tried to prepare ISO documentation with consultancy help, because there were no other similar practices to copy within Greek academic libraries. The library adopted ISO as a good practice from abroad.

In the ISO 17025 cases, adoption was associated first and foremost with coercive pressure. Findings from interviews supported the notion that there was normative pressure as well, suggesting a combination of coercive and normative pressure in five out seven cases. In the case with the EMAS quality management system, adoption was associated with normative and mimetic pressures. 
Normative pressure was derived from the project director's interests accompanied by the rector's support.

Table 10-2 ISO practices and type of isomorphic pressures

\begin{tabular}{|c|c|c|c|c|c|}
\hline \multirow[b]{2}{*}{ University } & \multirow[b]{2}{*}{ Case } & \multirow[b]{2}{*}{ ISO type } & \multicolumn{3}{|c|}{ Pressures } \\
\hline & & & Coercive & Normative & Mimetic \\
\hline \multirow[t]{11}{*}{ Ua } & 1 & $\begin{array}{l}\text { ISO } 9001 \text { for } 3^{\text {rd }} \text { time } \\
17025^{*}\end{array}$ & $x$ & $x$ & \\
\hline & 2 & ISO 9001:2000 & & $x$ & \\
\hline & 3 & ISO 9001:2000 & $x$ & & \\
\hline & 4 & ISO 9001 & $x$ & $x$ & \\
\hline & 5 & ISO $9001: 2000$ & $x$ & & \\
\hline & 6 & ISO 9001:2000 & & $x$ & $x$ \\
\hline & 7 & ISO 9002, 9001* & $x$ & $x$ & \\
\hline & 8 & $\begin{array}{l}\text { ISO 9002/94 (since } \\
\text { 1994), and } 9001^{*}\end{array}$ & $x$ & $x$ & \\
\hline & 9 & ISO 17025 & $x$ & $x$ & \\
\hline & 10 & ISO 17025 & $x$ & $x$ & \\
\hline & 11 & 17025 from 1997 & $x$ & & \\
\hline \multirow[t]{5}{*}{$\mathrm{Ub}$} & 12 & ISO $9001: 2000$ & $x$ & & \\
\hline & 13 & EN 45001/ ISO 17025 & $x$ & & \\
\hline & 14 & $\begin{array}{l}\text { EN 45001/ ISO } 17025 \\
\text { in process for renewal } \\
\text { and } 9001^{*}\end{array}$ & $x$ & $x$ & \\
\hline & $\mathrm{RC}$ & $\begin{array}{l}\text { ISO 9001:2000 from } \\
2000 \text {, renewal } 2005\end{array}$ & & $x$ & $x$ \\
\hline & 15 & ISO 9001:2000 & $x$ & $x$ & \\
\hline Uc & Library & ISO $9001: 2000$ & & $x$ & $x$ \\
\hline Ud & 16 & ISO 9001:2000 & & $x$ & \\
\hline \multirow[t]{2}{*}{ Ue } & Library & ISO $9001: 2000^{*}$ & & $x$ & $x$ \\
\hline & 17 & ISO 45001 & & Not in use & \\
\hline \multirow[t]{2}{*}{ Uf } & 18 & ISO 17025 & $x$ & $x$ & \\
\hline & 19 & ISO 9001:2000 & $x$ & $x$ & \\
\hline Ug & 20 & EMAS & & $x$ & $x$ \\
\hline Total & & & 15 & 17 & 5 \\
\hline
\end{tabular}

* in process 
In addition, EMAS reflected the university's prestige (mimetic); it "decorated" the university's website. This case reflects the entire university's quality management and therefore during my final integration phase (in chapter 11) it will be related to the meso level adoption of quality management.

Out of the wide variety of laboratories implementing ISO standards, ISO 9001:2000 and ISO 17025 were the most popular in engineering laboratories. Especially in one university, motivation to adopt ISO standards in engineering was supported by the university's rectorate in order to increase the university's competitiveness.

Only three cases showed coercive pressure, as ISO was required by a funding project and the directors held negative attitudes, in some respects, to this quality practice. Their interviews suggested that they believed this practice to be bureaucratic. Another director said, "in order to qualify for funding projects, we were obliged to participate in this process". He further commented that this perception regarding his laboratory's role is different than that of the market, which reflects his feeling coercive pressure (adopting ISO for reasons of funding but without e.g. normative conviction of its usefulness).

In cases where normative pressure was identified, directors emphasized the benefits of ISO practices in everyday operations. Moreover, they pointed out that ISO practices served as a guide to their personnel's operations and perceived that this practice was beneficial not only as a "fundraising technique", but also for academic research processes. The above findings do not suggest clear mimetic pressure, although in one case, a combination of normative and mimetic pressure was underlined. Some directors pointed out that "they always had a positive attitude about this application and the funding project helped them to accomplish this goal". Some pointed out that the cost (for ISO application and practice) was high; however, this was not a reason for declining adoption. A few others mentioned that adoption of ISO was a collaborative choice because some members in the laboratory participated in various networks and in special professional associations. In this study, I interpreted pressure as normative when the laboratories or academic support services participated in professional networks. In some ISO 9001 cases, laboratories were in the process of adopting the 17025 and vice versa in order to be more competitive and complete regarding their laboratory's quality. In ISO 17025 cases where ISO was required by the law and where normative pressure was in combination with coercive, the directors' comments concerned ISO benefits to the laboratory and to the university as a whole. In such cases, the unit's personnel were very satisfied with their participation in this quality management practice. In these cases, the interviewees' tone of voice and enthusiasm indicated support of this practice as 
well. When mimetic pressure was observed, interviewees were not sure about the ISO outputs and benefits and commented "we will see"; also they noted "ISO is associated with prestige".

Overall (i.e. regarding both laboratories and academic support services), most cases, 17 out of 23, involved normative and coercive pressures. Of all 23 interviews, only one pointed out that ISO registration had expired because their services were not connected anymore with the market needs. However, the director pointed out that the laboratory's staff still followed ISO requirements and that they had adopted the style of this practice. Even if their ISO application was no longer formally in place, quality practices and a quality culture in this laboratory were still present.

It merits attention that the hierarchy pyramid within Greek universities, commented on by a large number of interviewees, comes in antithesis to ISO requirements and is evaluated negatively for adoption of ISO. Participants mentioned obstacles which were connected with the decision-making structure, policies, and regulations that laboratories needed to follow as units of the university. On the other hand ISO certification required flexibility. Participants' comments were:

"Usually (ISO requirements as management practice) is in antithesis to the laboratory's hierarchy and requires personal involvement".

"Laboratory's director does not have the power to manage and moreover to plan and hire appropriate human resources (personnel). This is in antithesis to ISO requirements which needs strong management".

"It needs a lot of work regarding documentation and regulations, personnel need extra help and education in order to adopt this process, it is very bureaucratic and expensive and it is very difficult to square with the Greek public administration".

Additionally, the document review process (which I used for triangulation purposes) provided extra information regarding the university rectorate's motivation (in the case of $\mathrm{Ua}$ ). There were numerous reports and publications on this university's website. They supported the university's triple mission and indirectly, adoption of ISO. It is significant that one publication, "List of laboratories which provide services", presented the laboratories to a broad audience (presumably including market parties) as "the place of solution". It should be noted that the same university characterized itself as a "think tank" and a champion of research and development and has received awards at the European level. It has also provided the Code of Ethics and the law under which all these research units operated. In the same publication, we can see university's 
and research units' income for the years 1997 to 2005. This university published the research income budget on its webpage which directly related to its research capacity. Comments made by directors in this university connected adoption of ISO standards with the university's mission and quality. More specifically, one of them stated, "our university's mission is the triptych: education, research, and distribution of services and knowledge (spin off) to industry; thus ISO is parallel to our university's mission". Another pointed out: "ISO represents an inductive method in order to define quality in a university as a whole; in other words, if a university has various quality units it means that the university is perceived as high quality". Still another commented: "motivation starts from rectorate encouragement for laboratories' competitive advantage". Participants from the same university also exemplified other aspects of their rectorate's motivation technique or "light" pressure that helped them persist in adoption of ISO standards. For instance, one director commented, "We had an incentive by our rectorate suggesting increasing personnel and sources in case we applied for this standard (i.e., policy support such as acquiring a loan that we could repay in 5 or 6 years). By that time we would have enough income from this application".

\subsection{Summary and Conclusion}

The aim of this final empirical study was to examine isomorphic pressures that might influence the adoption of ISO standards as an example of quality assurance practices at the micro level in Greek higher education. Data revealed that ISO standards in laboratories and academic support services were applied in six Greek universities out of the 21 total, in the absence of an effective national quality assurance system ${ }^{37}$. To this number, I may add another university that adopted EMAS (Environmental Management and Accounting System; somewhat analogous to ISO) for the entire university's operation which means that it is related to the meso level rather than the micro level. Historically, the first ISO application in Greek higher education appeared in 1994, and several laboratories and Research Committees gained registration several times since. In addition, this empirical study indicated that neo-institutional pressures do matter in the adoption of ISO standards within Greek universities: the competitive environment gave rise to coercive and normative isomorphism in laboratories and normative and mimetic isomorphism in academic support services.

In this study, the rectorate's motivation and encouragement definitely played an important role in many participants' persistence in application of ISO, not only in one university (Ua) where this was a definite policy, but in other universities too

37 The quality assurance law (Law 3374/2005) by the end of 2006 was not in effect. 
$(\mathrm{Ub}, \mathrm{Uc}$, and Ug). The rectors' involvement could be perceived as "internal coercive pressure" and is related to university leadership practices and directly connected to the university's mission and vision. It seems that the leadership of the university could provide a coercive power strongly influencing the university's laboratories. However, in chapter 5, I mentioned that laboratories are autonomous units, and perhaps readers might perceive this as a contradiction: how it is possible for an independent unit to be affected by an internal pressure? However, academic laboratories are not completely "free" and "independent", because they are part of the whole university. Organizationally, by default, it is a unit of the university even though not part of the normal line hierarchy of faculties and departments; laboratories rely on the university's physical resources and staffing. Although laboratories might operate with apparent autonomy, they still come under two "leadership" models: the university's mission supported by the rector's leadership role and its own internal organization. There remains therefore, a rectorate-driven as well as mission-driven internal pressure for adoption of ISO standards. Additionally, as noted previously (chapter 5), many of the Greek laboratories are very profitable, through research grants and joint university and business-driven research. As such, it is within the financial interests of the university to attract, build, and support competitive laboratories since a percentage of the earnings of the laboratories would be returned to the sponsoring university's budget. Thus, an additional pressure exists for both the laboratory and the university: financial gain (a coercive pressure, in our conceptual framework).

Moreover, the extent to which organizational performance can effectively be improved through ISO standards remains an important issue. The revised ISO 9000:2000 series represented a real step forward in quality assurance, since it aimed for "customer satisfaction assurance", not just "product quality assurance", as Gotzamani and Tsiotras, (2001) and Hoyle (2003) discussed. In this chapter, ISO cases were found in libraries, research committees, and in laboratories. It seems that these units decided to satisfy their customers via ISO-certified services and products. However, adoption of an ISO certification as a valid indicator of high quality organizational performance needs further investigation. Universities are organizations that require multiple variables to assess their overall organizational performances in teaching, learning, research and knowledge transfer. Therefore, with ISO compliance active across only a few universities it seems difficult to imagine that this type of pressure will soon become a potential barometer of performance which could be broadly applied to all university's performance. 
During the process of this research, several laboratories were in the process of obtaining ISO certification (informal information via personal communication); however, it was impossible to obtain more details. The fact that more than 23 laboratories and academic support services implemented ISO or were in process of doing so is possibly a sign that the use of ISO, in the future, will motivate different levels of Greek higher education quality management practices ("domino effect"). However, performance improvement is not a direct function of adoption of ISO since certification relies primarily on structured laboratory performance of product standardization procedures and its management. Nevertheless, the adoption of ISO standards is a step in the direction of measuring and evaluating production and performance and thus focuses attention upon potential benefits of quality management systems. Hence, it becomes a step towards adopting improvement-oriented practices across the entire organization. As already noted, there is progress; although slow, as universities begin to adopt and require quality management practices in teaching, learning, and services. 


\section{Integration and Reflection: Neo-Institutional Approach, Quality Management, and Greek Higher Education}

\subsection{Introduction}

Quality management deals with policies, systems, and processes designed and implemented to ensure the maintenance and improvement of quality in organizations including universities. Quality management suggests a transformation process requiring a fundamental shift in management practice and culture. In Greek higher education and at least until 2007, quality management was in an early and debated stage. The purpose of this study was to examine the adoption or non-adoption of quality management within the universities in Greece as an outcome of organizational change practices. Thus, the main problem statement of this study was to identify relationships between the organizational factors for stability and/or change in Greek universities and the universities' adoption (or lack of adoption) of quality management. To address this problem, I divided it into three sets of sub-questions. The first question was: What do we understand by quality management in higher education institutions? I approached this question through a review of quality management in higher education, including the European University Association's Institutional Evaluation Programme (EUA-IEP), the Malcolm Baldrige Education Criteria for Performance Excellence (MB), and quality approaches following ISO standards. Additionally, I used quality management practices from abroad, which are reported in chapter 2, as a generalized mirror to compare how quality management theories work in actual practice in higher education in Greece where this current study was situated. The second question was: Which organizational factors for stability and/or change concerning the adoption of quality management can be observed in the literature on organizations and higher education institutions? This question led me to utilize open system theory and neo-institutional theory in order to provide me with the best understanding of the phenomena. Within neoinstitutional theories, isomorphism is the concept that was most useful to study whether and why Greek universities were following (mimicking?) international examples. From a neo-institutional view, organizations, which include universities, operate in an environment dominated by rules, requirements, understandings, assumptions, beliefs, and procedures (scripts) about what constitutes appropriate or acceptable organizational forms and behavior. In neoinstitutional theory (DiMaggio \& Powell, 1983), three mechanisms were identified 
through which isomorphic change occurs: coercive forces, which stem from political/legal influence and the problem of legitimacy; mimetic forces resulting from standard response to uncertainty; and normative forces associated with professionalization. Since the objective of my study was higher education institutions, I focused on the use of neo-institutional perspectives in empirical studies of organizations as well as studies undertaken in the field of higher education. This theoretical pathway led me to single out normative, mimetic, and coercive pressures as the main independent variables in order to explain adoption (or non-adoption) of quality management in Greek higher education institutions.

Next to the theories addressing organizations as a whole, universities were characterized as complex organizations with many layers; therefore, I singled out the universities' characteristics that operate as intra-organizational elements, which could provide the best insight into the processes of adoption of quality management. In the case of the Greek universities and their state-dominated context, some organizational characteristics such as their mission and decisionmaking processes were equal across all universities; as such, those characteristics were ruled out as explanatory variables. The key variables from the intraorganizational characteristics remaining and used in this study fell into six categories: leadership, vision, age, size, location, and range of studies (chapter 3).

This study utilized the conceptual framework (figure 11-1) developed in chapter 3 , which was created from a review and synthesis of the literature on systems theory, neo-institutional theory, universities' characteristics, and quality management (chapters 2 and 3). The variables enclosed in the conceptual framework were operationalized in chapter 4 .

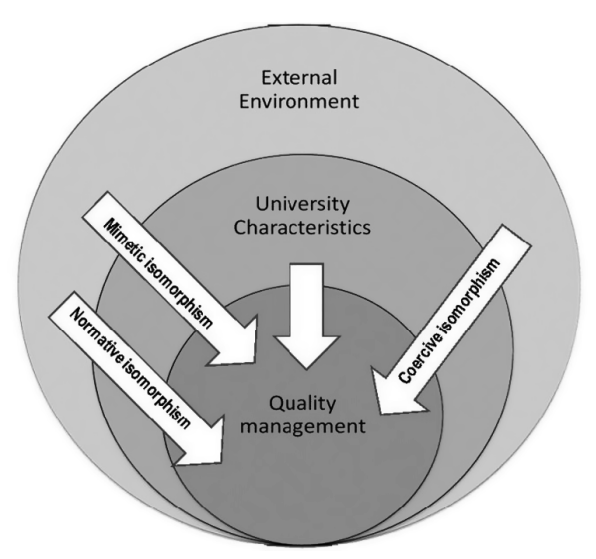

Figure 11-1 Conceptual framework 
The third research question guided the empirical studies of this book and divides into two parts. The first part asks: Which organizational factors for stability and/or change concerning the adoption of quality management can we find empirically in Greek higher education? This question led to a search through several empirical studies for the three types of isomorphic pressures as well as for organizational characteristics actually existing in and around Greek universities. The second part asks: Do these isomorphic pressures (coercive, normative, and mimetic) differ at macro (higher education system), meso (individual universities) and micro levels (academic departments, laboratories, and academic support services)? The specification of the third research question into different levels arose out of the realization that higher education institutions are complex organizations with multiple levels; moreover, they exist in a multi-level, multi-actor environment. Hence, a single, uniform explanation at these different levels should not be assumed.

Besides the theoretical issues that any empirical social scientist must address, additional challenges were posed by the choice of Greek higher education as the object of study, which had to be taken into consideration when designing the empirical phase of this research. In Greece, the environment for conducting almost any type of empirical social science research has been characterized internationally as notoriously difficult. Moreover, the hot debate on quality assurance in higher education, which took place in Greece during the period of the study, made it even more difficult to study quality management directly.

For these reasons, and others mentioned in chapter 4, a sophisticated approach to the empirical study became necessary and a multi-level mixed design was chosen. The research design thus involved mixing qualitative and quantitative approaches at three levels (macro, meso, and micro) for the purpose of completeness. Like a scaffold, each empirical study of this research was built on and was designed to harmonize with others. The empirical study took place over a two-year period from 2005 to 2007 (figure 11-2).

As figure 11-2 illustrates, the first studies took place at the macro level of the whole higher education system. As a preliminary study, an environmental scan was undertaken (chapter 5). Then, in chapter 6, the 'hot debate' around quality management in Greek higher education was highlighted during one of the 'hottest' moments of the debate through a study of how the press addressed quality assurance. That debate centered on the Bergen follow-up meeting from the Bologna Process (May 2005) and it constituted isomorphic pressure on Greece's policy-making that was mixed in tone (positive as well as negative), using mainly normative isomorphic arguments. I argued that the dominant 


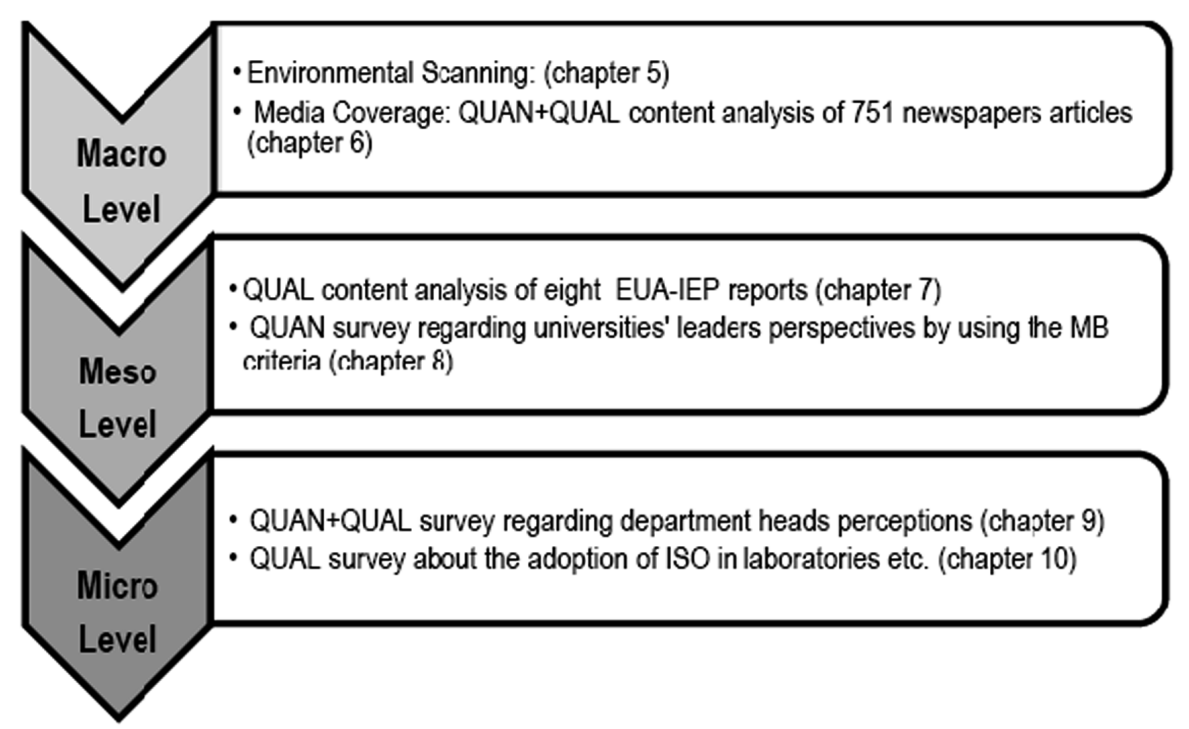

Figure 11-2 Presentation of the Empirical Studies

meso level of universities as an organizational entity. In chapter 7, the major question studied there included which circumstances (isomorphic pressures and university characteristics) would cause universities to engage voluntarily in external reviews; the EUA-IEP was a prime example to gauge involvement. A more direct approach at measuring changes was attempted in chapter 8 , when questionnaires were sent to university leaders based on the internationally recognized Malcolm Baldrige (MB) quality criteria. This approach intended to elicit any pressures on, and characteristics of, universities in relation to their stage of development in quality management. At the micro level, the last two studies more or less mirrored those at the meso level: one was accomplished through a questioning approach (chapter 9) and another one in chapter 10, researched cases of 'good practice' (at least in comparison with others in Greece). At that stage of my investigation, questionnaires were directed at department heads; I was able to use their responses to triangulate some of the views of university leaders. That 'good practice' involved searching and studying units (laboratories and some university services) that applied quality management according to ISO standards. As in chapter 7 , the question in the study on ISO-applying units was which environmental pressures and/or organizational characteristics set them apart from the non-adopters of these types of quality management practices.

The principal aim of this chapter is to bring together the empirical findings answering research question three. The task of integrating the inferences from the different studies will start in a bottom-up manner. Initially, I will address the 
second part of the third research question by focusing first on each of the different levels of the higher education system. Then, in the following sections, $(11.2,3$, and 4), I will combine the results from all the empirical studies by each level previously identified. After that, in section 11.5, I will venture to answer the first part of the of the third research question by identifying organizational factors for stability or change across the different system levels.

This chapter also has interrelated goals: to form an improved version of the conceptual framework (Figure 11-1), and more broadly to reflect on the fourth research question: Does the empirical evidence on the adoption of quality management in Greek universities coincide with the theoretical approaches articulated in this study? That answer will be addressed in section 11.6.

Since this study employed a multi-level mixed methods design, the present chapter returns to the issue of inference quality in mixed methods studies. Specifically, the interpretative rigor considerations of Tashakkori and Teddlie (2003) and Teddlie and Tashakkori (2009) that were introduced in chapter 4, will now be answered. That discussion forms the subject matter of section 11.6.

Finally, this chapter includes a section on the future avenues of quality management research in Greek higher education and concludes with recent changes and future challenges in Greek higher education.

\subsection{Macro Level Views on Isomorphism and Quality Management}

This section addresses the first, macro level, of the third research question, i.e. Which organizational factors for stability and/or change concerning the adoption of quality management can we find empirically at the macro level in Greek higher education? The understanding of this level was constructed mainly from two studies: one was the environmental scan of Greek higher education (chapter 5) using documents and observations, and which also operates as background information for the entire study; the other was a qualitative and quantitative content analysis of printed media (chapter 6). The latter study addressed public images of adoption of quality management and isomorphic pressures in Greek higher education during the peak period of the quality assurance debate. That study used newspaper coverage of the issue in May 2005, when higher education ministers gathered in Bergen to discuss the follow-up of the Bologna Process. That was also the period when the first quality assurance law came before the Greek Parliament. 
Data from the environmental scan indicated that Greek higher education institutions operated in a legalistic environment and operated under a plethora of law and regulations established by a highly politicized state bureaucracy. All public universities are awarded permission to operate by the Ministry of Education and are state funded institutions. Only as recent as 2005, the Greek Government established a national system for quality assurance in higher education. In addition, the environmental scan showed relevant elements from the attitudes of Greeks and Greek culture; although neither one was diametrically opposed to the concept of quality management, they each required significant adaptation to quality management in both the private and public sectors (including universities).

A content analysis surveyed how newspapers covered the proposed law and how they presented quality assurance in the press by using agenda setting concepts in combination with the idea of isomorphism: the main thesis was that media influence what audiences put on their agenda, rather than the precise content of their points of view. Nevertheless, media might influence the discussion by providing certain types of arguments. Framing those arguments in terms of neoinstitutional theory, they were categorized as normative, coercive, or mimetic pressures for isomorphism. Qualitative and quantitative analysis from 251 inputs from 54 newspapers revealed that quality management, as such, was reflected in only 9 inputs, while the quality assurance law, received 246 inputs, which was the chief topic. Concerning isomorphic perspectives, over one third $(35 \%)$ of the quality assurance law inputs expressed coercive pressure, $28 \%$ expressed normative pressure, and less than $14 \%$ dealt with mimetic pressure, while the remaining $20 \%$ were in the combination categories of normative and/or coercive and/or mimetic pressures. The findings show that newspapers carried mostly coercive statements on the quality assurance law.

During May 2005, overall, $47 \%$ of all inputs in the study displayed a negative tone toward the image of quality assurance, while $41 \%$ were mainly positive, and $11 \%$ neutral. More than 55\% of entries expressing coercive pressure had a negative tone (48 out of 86 ). Articles in the combination categories, also in a majority, had a negative tone $(67 \%)$. In contrast, a positive tone was found in 39 out of 72 inputs characterized as giving normative pressure (54\%). Evidence of a positive tone $(47 \%)$ was also found in newspaper entries with arguments giving mimetic pressure.

The findings at the macro level invited additional considerations. Until 2005, quality assurance, evaluation, and similar problems had been addressed almost continuously at the governmental level (Asderaki, 2009; Billiris, 2004; Kyriazis \& 
Asderaki, 2008; Stamoulas, 2006; YPEPTH, 2003, 2005, 2007). Asderaki (2009, p. 113) noted that "in the early 1990s an attempt was made to introduce institutional or departmental evaluation (article 24 Law2083/1992, 21.09.1992) but met strong reactions from the opposition political parties and universities as well". Thus, quality assurance policy was seeking to find "an open window". Kingdon (1995, p. 165) defined a policy window as "an opportunity for advocates of proposals to push their pet solutions, or to push attention to their special problems". In Greece, this opportunity came with the Bologna Process's stocktaking exercise. The general consensus was that 'Bologna' was the main reason that drove the Greek Government to develop the quality assurance policy for its higher education institutions. My notions of isomorphic pressures arising from the Bologna Process are supported by Ravinet (2008) when she commented that follow-up activities and the monitoring of progress on the Bologna Process action lines in participating countries through the stocktaking exercise "allow comparisons, and create effects on socialization, imitation, and shame-which can be powerful means of coercion". She noted that the mechanisms "are even more effective because they are made legitimate by the myth of the Bologna Process as a mode of voluntary [participation]" (p. 365). Asderaki (2009, p. 116) reported that the draft traffic-light scorecard was sent to the Ministry of Education in March 2005. It illustrated the 'negative image' of the country, with red-colored indicators ( 1 on a scale of 5 ). "This made the leadership of the ministry realize that there was no time left for delays and that decisions concerning the legislative framework for the establishment of the national quality assurance system had to taken... the Minister presented the draft law for quality assurance before the Bergen Ministerial conference (2005) and the country scorecard was improved" (Asderaki, 2009, p. 116): in the published version of May 2005 Greece's quality assurance dimensions appeared having scores 3 and 4 on a scale of 5. From this perspective, the adoption of a quality assurance law in Greece was predicated upon an "open window". This window had opened before, namely around the follow-up conferences in the Bologna Process of 2001 and 2003. However, in those years the windows opened and closed without causing movement towards a policy change; perhaps that was because no public shaming threatened at those occasions since the scorecards were not published before 2005 .

Distinguishing the three neo-institutional mechanisms allows additional insight into what drove the adoption of the quality assurance law at the macro level in Greek higher education. Coercive pressure was out of the question since the Bologna Process is a voluntary international process, where no lawmaking or sanctioning by a supranational authority is involved. However, its pressure was strong (Asderaki, 2009; Kyriazis \& Asderaki, 2008). The stocktaking exercise's 
easily understood graphic display of a scorecard came perhaps close to a coercive effect on national Greek pride. In my conceptualization, it remains a mimetic pressure, however strong the impact of public shaming might have been.

Focusing on the other two pressures (normative and mimetic) DiMaggio and Powell (1983, p. 151) noted that "companies adopt these 'innovations' [in this case, quality assurance-AP] to enhance their legitimacy, to demonstrate they are at least trying to improve". The basic goals of the Bologna process, which included cooperation in quality assurance, were agreed upon by the ministers who were responsible for higher education in 1999. The regular follow-up meetings and the multitude of working groups and seminars to prepare them, created active networks between ministers, civil servants at education ministries and other experts which were explicitly meant to create, disseminate and harmonize knowledge and practices (Westerheijden et al. 2010). Thus, this aspect of the Bologna Process can be seen as normative pressure at the macro level.

Networking and prevalence notwithstanding, it remains questionable whether normative pressure statements that appear in the content analysis were an actual and decisive pressure at the macro level. In my analysis, mimetic pressure was paramount and influenced the change in policy regarding quality assurance, namely through the stocktaking exercise, as noted above. Radaelli (2000, p. 29) mentioned that "given the level of uncertainty that pervades EU policy-making, it can be argued that policy transfer should follow the path of mimetic isomorphism in many circumstances". The colourful mosaic that showed progress was a reason for the policy change in Greece (Asderaki, 2009), where the threat of public pressure may have been more important than the professional arguments on how best to organize quality assurance.

Table 11-1 summarizes, by approximation, the role that the types of isomorphism played at the macro level in Greek higher education concerning adoption of quality management.

Table 11-1 Isomorphic pressures at macro level in Greek higher education system

\begin{tabular}{cccc}
\hline & Coercive & Normative & Mimetic \\
\hline Macro level & - & - & ++ \\
\hline Legend: - type of pressure absent; + type of pressure present; ++ type of pressure strongly present.
\end{tabular}

However, when mimetic isomorphism is paramount, it is possible that the adoption of quality management is simply "myth and ceremony" meant to 
achieve legitimacy rather than to bring about actual change (Meyer \& Rowan, 1991). Their comment may reflect the "window of opportunity" approach. Kingdon (1995) had already noted that it was not clear if policy adoption reflected success in policy implementation. Tolbert and Zucker (1983, p. 27) also indicated that "legal requirements do not always ensure adoption". In other words, the adoption of the law might happen, but would that lead to actual adoption of quality management practices inside universities?

\subsection{Meso Level Views on Isomorphism and Quality Management}

In chapters 7, 8, (and partly also in 9 and 10), I addressed issues on isomorphism, adoption of quality management, and universities' characteristics at the level of universities as a whole. Each empirical study was designed to provide appropriate information to answer the meso level (individual universities) of the second part of the third research question. In order to construct an overall meso level view, the findings from these empirical studies will be summarized and discussed here under two headings: documented quality management and leadership's perceptions. I call documented quality management the data that derived from written documentation on quality management practices such EUAIEP and Eco-Management Systems (EMAS). In addition, leadership perceptions derived from the surveys that formed the basis of the studies reported in chapters 8 and 9. This section concludes with bringing together isomorphism, universities' characteristics, and adoption (or not) of quality management at the meso level in section 11.3.4.

\subsubsection{Documented Quality Management}

\section{Evaluator's Reports on Isomorphism and Quality Management}

The EUA-IEP was a voluntary quality management practice, in which eight out of 21 universities in Greece had participated by 2006. Chapter 7 contains a qualitative content analysis of the eight EUA-IEP evaluators' reports. The eight universities that participated in EUA-IEP represented $45 \%$ of the total student body and $58 \%$ of the Greek faculty members' body. The EUA-IEP reports yielded observations on three themes: isomorphic pressures, university characteristics, and quality management, as highlighted in the conceptual framework of this study. Data of the qualitative content analysis (chapter 7) identified isomorphism influencing the participation in the EUA-IEP.

Evidence from the reports indicated that all eight universities that participated at the EUA-IEP were seeking feedback about their overall performance. Quality 
management as a formalized, centralized, or complex practice was absent in all eight universities. However, the majority of the reports demonstrated that there were some quality assurance related activities in regards to students' evaluations, but without formalized follow-up activities. The analysis suggested that these activities were organized in individual departments, which signalled the autonomous status of Greek academic departments. Overall, the EUA-IEP reports implied that quality management in Greek universities was not a routine practice, at least up to 2006.

The results of the qualitative content analysis identified isomorphism as influencing the participation in the EUA-IEP. Coercive pressure did not play a significant role in the decision to invite the EUA-IEP. Normative pressure was indicated in this chapter's study by the fact that the rectorate's management commitment was needed to be eligible for the EUA-IEP and by the fact that these universities participated in a professional association, i.e. the EUA. Regarding mimetic pressure, the university characteristic of location might be associated with this type of isomorphism, because only peripheral universities participated in the EUA-IEP. It seems that peripheral universities in Greece wanted to use the EUA-IEP as a compensating policy to gain prestige and legitimacy against the well-established universities in Athens.

Regarding the university characteristics in this study, 'leadership' is one of the premises of the quality management literature, which appeared specifically relevant in the EUA-IEP, since a leadership decision was the catalyst for engagement in the EUA-IEP. Leadership in these eight universities was one of two university characteristics that correlated with having participated in the EUA-IEP (the other was location).

Isomorphism and the use of Eco-Management Systems in Greek Universities

Chapter 10 contains a qualitative study to examine the isomorphic pressures that influenced units inside universities to adopt quality management practices. The empirical study reported in chapter 10 was designed to collect data from the micro level; however, I found that one university as a whole (thus at the meso level) had implemented an environmental quality management system, the European Eco-Management and Audit Scheme (EMAS). Data revealed that the adoption of this particular quality management system was related to normative pressure (the project director's interests and knowledge from abroad) and accompanied by the rector's support (leadership involvement). In addition, adoption of EMAS reflected on the university's prestige (mimetic pressure), shown by the fact that the EMAS symbol decorates the university's website along 
with the EUA-IEP sign, in all appearance as a status symbol to show or gain legitimacy.

\subsubsection{Perceptions about Isomorphism and Quality Management}

In this section, questionnaires gathering the actors' perceptions concerning isomorphism and adoption of quality management were designed to provide answers to the meso level research question. Data came from two empirical studies, reported in chapters 8 and 9. For the study in chapter 8 , I sent a questionnaire via regular mail to all rectors and vice-rectors in Greek universities. The questionnaire, which followed the quality criteria from the MB (see Appendix B), studied on the one hand leaders' perceptions in relation to isomorphic pressures and on the other hand, the $\mathrm{MB}$ enabled finding out the extent to which quality management processes had been implemented at the meso level. The picture concerning isomorphism and adoption of quality management at the university level was completed by using some parts of an electronic survey that was distributed to the departments' heads in Greek higher education (chapter 9).

\subsubsection{University Leaders' Perceptions about Quality Management}

In chapter 8, the MB-based survey was used to gather knowledge on how leaders (rectors and vice-rectors) in Greek universities perceived isomorphic pressures and to what extent they adopted quality management practices to improve their university's performance. The MB quality award was designed originally in the USA to help organizations use an aligned approach to organizational performance management that results in delivering ever-improving value to students and stakeholders, contributing to marketplace success, improving overall organizational effectiveness and capabilities, and fostering organizational and personal learning. Blazley et al. (2003, p. xi) highlighted that "MB and related scoring guidelines are powerful assessment instruments that will help leaders of educational organization identify strengths and key areas for improvement. Leaders then need to use the information to achieve higher levels of performance". The core values and concepts of the MB criteria are embodied in seven categories: 1. Leadership, 2. Strategic Planning, 3. Student, Stakeholder, and Market Focus, 4. Measurement Analysis and Knowledge Management, 5. Faculty and Staff Focus, 6. Process Management, and 7. Organizational Performance Results. The MB criteria were used in my questionnaire on the one hand to observe a leader's perception, while on the other hand to understand the extent to which quality management practices were implemented in universities. It also let 
me ask leadership's perspectives on performance improvement in relation to isomorphic pressures.

In this empirical study, data was derived from nine universities out of the 20 listed at that time by the Greek Ministry of Education. The MB questionnaire provided the quantitative data on normative and mimetic isomorphism. (Coercive pressure was not included in this survey, as the quality assurance law was submitted to the Greek parliament for discussion only in the period when the survey was held, i.e. May 2005). Data indicated that both normative and mimetic pressures were perceived as high in almost all nine cases and there was almost no inter-university variation between these pressures. It was, first of all, interesting that all universities were seen to be in a very similar situation. However, due to the virtual absence of variations in normative and mimetic pressures, further analysis on the influence of different pressures on different levels of quality management development could not be performed.

I used two ways to examine the adoption of quality management, since formalized quality management was almost absent in Greek universities until 2005. I did that in order to collect as much data as possible so that less formalized quality management practices could be found. One question posed in the first part of the MB survey addressed the presence of a quality management system as a whole. All answers to that question were negative; however, the MB survey instrument also allowed collecting more detailed information about separate practices that could be regarded as quality management practices. The activities were there, but in a disjointed fashion, so that there was not a "system" of quality management in the respondents' eyes. All of this information was integrated into an overarching assessment of the university's quality management (QM) stage, which ranged from lacking (L), to embryonic (E), developing (D), and advanced (A). The data charted in Table 11-2 shows the quality management stage at the meso level from leaders' perspectives.

Table 11-2 Leaders' perspectives on quality management stage at the meso level

\begin{tabular}{cccccccccc}
\hline & U1 & U2 & U3 & U4 & U5 & U6 & U7 & U8 & U9 \\
\hline QM stage & D & L & A & D & E & E & D & E & D \\
\hline
\end{tabular}

Legend: L: lacking quality management, $E$ embryonic, $D$ developing, and $A$ advanced quality management

Only one university's leader indicated that his university's quality management was 'lacking'. Three universities' quality management practices were regarded as 'embryonic'. Four were in a 'developing' stage according to the MB criteria. One 
university (U3) achieved an 'advanced' stage of quality management according to its leadership.

Additionally, when converting the leaders' responses to the MB survey into "hypothetical scores" according to the MB quality award criteria, it appeared that the universities were not completely "black sheep" concerning quality management practices (Appendix Table B4). There are, therefore, at least a number of "white spots" on the way towards excellence in Greek universities and it seemed that it was a kind of quality movement at the meso level.

Upon closer inspection of the response patterns, the Category 6 "Process Management" stood out as the category with most quality management-related practices implemented. Five out of nine universities reported an implementation score of $63 \%$ or more in this category. Explaining this high score, one university leader noted that some of the items in the category "Process Management" were related to requirements derived from the higher education law (1268/82). This statement shows that the existing regulative arrangements exerted some coercive pressure, even before laws explicitly started to address quality assurance. Moreover, university leaders might see "process management" as a means to gain control over the behavior of the (otherwise very autonomous) professors. Whether such control might be real or perceived, it could explain the leaders' preference to organize practices in this category, rather than allowing their own behavior to be controlled by formalizing strategic planning into quality management-related practices (Strategic Planning is addressed as Category 2 of the MB criteria and obtained much lower scores).

In this empirical study, I also checked against the control variables of universities' characteristics, but no correlations were found between the quality management stage and university characteristics of age, size, location, and range of studies.

Since in the previous study on the EUA-IEP, leadership emerged as a critical university characteristic, I gave special attention to leadership aspects in the MB questionnaire. The MB categories 1, 2, and 3 together are called the "leadership triad". These categories emphasize the importance of a leadership focus on strategy, students, and stakeholders. I recoded the responses of these MB categories into four ranges: "early stage of transition" (1), "beginning of systematic approach" (2), "systematic fact-based process in some part of the organization" (3), and "well integrated" (4). The data charted in Table 11-3 shows the leadership excellence and quality management stage at the meso level from leaders' perspectives. (For methodological purity, I also calculated an alternative QM-variable, using only the four categories of the questionnaire not included in 
the leadership, but the differences with the overall QM stage were in all cases less than 95 points, so that the recoded four stages remained the same.)

Table 11-3 Leadership Excellence and QM stage at the meso level

\begin{tabular}{cccccccccc}
\hline & U1 & U2 & U3 & U4 & U5 & U6 & U7 & U8 & U9 \\
\hline $\begin{array}{c}\text { Leadership } \\
\text { Excellence }\end{array}$ & 3 & 1 & 3 & 3 & 2 & 2 & 3 & 2 & 3 \\
\hline QM stage & D & L & A & D & E & E & D & E & D \\
\hline
\end{tabular}

Legend: 1 "early stage of transition", 2 "beginning of systematic approach", 3 "systematic fact-based process in some part of the organization", and 4 "well integrated"; L: lacking quality management, $E$ embryonic, D developing, and A advanced quality management

When the leadership excellence was in the "early stage of transition" (1), it correlated with the lacking stage; and conversely, "systematic fact-based process in some part of the organization well integrated" (3) leadership excellence coincided with advanced and developing quality management. It seems again, as in chapter 7 , that leadership makes a difference.

\subsubsection{Department Heads' Perceptions about Quality Management}

The survey of department heads, reported in chapter 9, mainly targeted the micro level (university departments), however, one question was related to the university (meso) level and is therefore relevant in this section. I asked department heads how they understood the quality assurance practices in their universities. Data regarding the university's quality management stage derived from responses by 22 department heads from 11 universities, only partly overlapping with the nine from which leaders had responded (Table 11-4).

Table 11-4 Department heads' perceptions about their universities quality management

\begin{tabular}{rrrrrrrrrrrr}
\hline & U4 & U6 & U7 & U9 & U11 & U14 & U15 & U16 & U17 & U19 & U20 \\
\hline QM stage & D & D & E & E & E & E & D & E & E & D & E \\
\hline
\end{tabular}

Legend: L: lacking quality management, $E$ embryonic, $D$ developing, and $A$ advanced quality management

In five universities (U6, U9, U11, U14, U16), I received responses from more than one department head an indicator of the quality management stage was calculated taking the average of those department heads' perceptions. In four of these universities (U6, U9, U11, U14), department heads held different perceptions of their university's state of the art concerning quality assurance. In the one university (U9), where even five department heads responded, two 
reported that their university's quality assurance was lacking, one perceived it to be embryonic, and the other two thought it was in the developing stage. Each department head appears to have their own, unique perception of quality management practices in their university. With all these provisos, Table 11-4 shows that according to department heads seven of these 11 universities were in the embryonic stage of quality management; the other four universities were in the developing stage.

\subsubsection{Integration of Leaders' and Department Heads' Perceptions on Quality Management}

In this section, the overall variable for the quality management stage was built upon the perception of rectors, vice-rectors, and department heads, and Table 115 presents the overall results from this approach. Where necessary (U6, U7, U9) I rounded up the average, joint judgements to 'developing'. This table provides a nearly equal distribution of cases across the four categories although admittedly, it may give a somewhat rosy picture of the actual situation.

Table 11-5 Overall quality management stage combining views from university leaders and department heads

\begin{tabular}{lcccccccc}
\hline & U1 & U2 & U3 & U4 & U5* & U6* & U7 & U8 \\
\hline Leaders & D & L & A & D & E & E & D & E \\
$\begin{array}{l}\text { Department } \\
\text { Heads }\end{array}$ & - & - & - & D & - & D & E & - \\
Overall & D & L & A & D & E & D & D & E \\
\hline & U9* & U11 & U14* & U15* & U16* & U17* & U19* & U20 \\
\hline $\begin{array}{l}\text { Leaders } \\
\text { Department }\end{array}$ & D & - & - & - & - & - & - & - \\
Heads & E & E & E & D & E & E & D & E \\
Overall & D & E & E & D & E & E & D & E \\
\hline
\end{tabular}

Legend: L: lacking quality management, $E$ embryonic, $D$ developing, and $A$ advanced quality management *EUA-IEP participants

One university was in the lacking stage (U2), seven universities were in the embryonic stage (U5, U8, U11, U14, U16, U17, and U20), another seven in the developing (U1, U4, U6, U7, U9, U15 and U19), and one in the advanced quality management stage (U3). Out of the eight universities that participated in the EUA-IEP (universities with an asterisk-*- in Table 11-5) four universities were found to belong overall in the embryonic stage category (U5 $\left.5^{*}, \mathrm{U} 14^{*}, \mathrm{U} 16^{*}, \mathrm{U} 17^{*}\right)$. 
The other four were in the developing stage category, according to their leaders' and department heads' perceptions.

Two university leaders who reported that their university's quality management was in the embryonic stage were from universities that had already participated in the EUA-IEP (U5*, U6*). Of the five department heads who reported that their university quality assurance system was in the embryonic stage, four were from universities that had already participated in the EUA-IEP (U9*, U14* U16*, and $\left.\mathrm{U} 17^{*}\right)$.

The overall results of the study as derived from chapters 8 and 9 surveys concerning the quality management developmental stage across the 16 universities are displayed in Table 11-6.

Table 11-6 Overall quality management stage as derived from Chapters 8 and 9 surveys

\begin{tabular}{lc}
\hline \multicolumn{1}{c}{ QM Stage } & Total \\
\hline Lacking & 1 \\
Embryonic & 7 \\
Developing & 7 \\
Advanced & 1 \\
\hline Total & 16 \\
\hline
\end{tabular}

Originally, I had planned to triangulate the data provided by university leaders (chapter 8) and department heads (chapter 9) from the same university with information from the EUA-IEP (chapter 7). However, to employ this process, I would have needed data for all universities and for each type of data collection that I used. Data were only sporadic and therefore, I could not perform this analysis.

\subsubsection{Meso Level Views of Isomorphic Pressures: Leaders' and Department Heads' Perceptions}

In this section, data from two studies (chapters 8 and 9) are integrated in order to identify the level of isomorphic pressures (low, medium, or high) that derived from rectors, vice-rectors and department heads' perceptions. The construction of robust overall variables regarding isomorphic pressures involved combining answers to questions on different issues in need of attention (pressure to act). All university leaders experienced high levels of both normative and mimetic 
pressures, ${ }^{38}$ except two, but those exceptions may be ascribed to their answering 'no opinion' to many of the relevant questions.

The survey of department heads in chapter 9 identified isomorphic pressures that the department heads felt to adopt the quality assurance system in their department. I assumed that the same level of pressure as they felt for their department would apply to their universities as a whole, to make an estimate of the pressures on a larger number of universities than had been reached by the leadership questionnaire in chapter 8. Data revealed that department heads felt a pressure, mostly mimetic, to some degree coercive, ${ }^{39}$ and a lesser degree of normative pressure. The low number of respondents precluded more sophisticated analyses of differences among department heads across universities or fields of knowledge.

The overall view of the normative and mimetic pressures at the meso level in Greek higher education can be seen in Table 11-7. Two non-parametric tests (Mann-Whitney $U$ and Wilcoxon $W$ ) were performed for each of these measurements. Both tests showed that mimetic pressure tended to be higher on monothematic universities than on more comprehensive institutions. I tested a number of control variables such as age, size, and location, but did not find additional correlations.

Table 11-7 Normative and mimetic pressures at the meso level, combining views from university leaders and department heads

\begin{tabular}{|c|c|c|c|c|c|c|c|c|}
\hline Overall & U1 & $\mathrm{U} 2$ & U3 & U4 & U5* & U6* & U7 & U8 \\
\hline Normative & $\mathrm{H}$ & $\mathrm{H}$ & $\mathrm{H}$ & M & $\mathrm{H}$ & $\mathrm{H}$ & $M$ & $\mathrm{H}$ \\
\hline \multirow[t]{2}{*}{ Mimetic } & $\mathrm{H}$ & $\mathrm{H}$ & $\mathrm{H}$ & $\mathrm{M}$ & $\mathrm{H}$ & $\mathrm{M}$ & $\mathrm{H}$ & $\mathrm{H}$ \\
\hline & U9* & U11 & $\mathrm{U}_{1} 4^{*}$ & U15* & U16* & U17* & U19* & U20 \\
\hline Normative & $M$ & L & L & $\mathrm{H}$ & L & $\mathrm{L}$ & L & $\mathrm{H}$ \\
\hline Mimetic & $M$ & $\mathrm{~L}$ & $\mathrm{~L}$ & $\mathrm{H}$ & $M$ & $M$ & $M$ & $\mathrm{H}$ \\
\hline
\end{tabular}

In this way, tentative measures have been constructed for all universities that took part in one or more of my studies, enabling a final analysis of 16 universities, instead of the smaller numbers of the separate studies.

38 There was no significant source of coercive pressure at the time university leaders were surveyed.

39 The study in chapter 9 took place in 2007 when the quality assurance law formally had come into force; therefore, there was a clear potential source of coercive pressure in this particular study. 


\subsubsection{Bringing together Isomorphism, Quality Management and University Characteristics}

In this section, I bring together isomorphism, universities' characteristics and quality management at the meso level. The overall views developed above (Table 11-7) were used for an overall analysis of isomorphism that Greek universities felt to adopt quality management. I developed the following Table 11-8 in order to find correlations between isomorphism (normative and mimetic) and quality management stage. Here, I did not calculate the coercive pressure, as the quality assurance law was not enforced until the very last of the studies brought together here.

Correlations between isomorphic pressures (normative and mimetic) and the university's quality management stage were not found. The pressures that the universities faced to adopt quality management practices varied to some extent but these variations were not clearly connected to the quality management development stage. The issue may be that so many universities reported high isomorphic pressures. Accordingly, high normative pressure was observed across all quality management stages (lacking, embryonic, developing and advanced). A similar picture pertains to mimetic pressure: I also observed high mimetic pressure combined with all quality management stages.

Table 11-8 Isomorphism and quality management at the meso level

\begin{tabular}{lcccc}
\hline \multicolumn{5}{c}{ Normative Pressure } \\
\hline QM stage & Low & Medium & High & Total \\
Lacking & - & - & 1 & 1 \\
Embryonic & 4 & - & 3 & 7 \\
Developing & & 4 & 3 & 7 \\
Advanced & - & - & 1 & 1 \\
Total & 4 & 4 & 8 & 16 \\
\hline & & Mimetic Pressure & & \\
\hline QM stage & Low & Medium & High & Total \\
Lacking & - & - & 1 & 1 \\
Embryonic & 2 & 2 & 3 & 7 \\
Developing & & 4 & 3 & 7 \\
Advanced & - & - & 1 & 1 \\
Total & 2 & 6 & 8 & 16 \\
\hline
\end{tabular}

University characteristics of age, size, location, range of studies, and leadership might contribute to adoption of quality management as control factors. In the 
following analysis I will summarize how the quality management stages (lacking, embryonic, developing, and advanced) at the meso level in Greek higher education were related to the university characteristics age, size, location, and range of studies (Table 11-9).

Table 11-9 Quality management at the meso level in Greek higher education as related to university characteristics Age, size, location and range of studies

\begin{tabular}{|c|c|c|c|c|}
\hline \multicolumn{5}{|c|}{ University Age } \\
\hline QM stage & Old & & New & Total \\
\hline Lacking & 1 & & - & 1 \\
\hline Embryonic & 5 & & 2 & 7 \\
\hline Developing & 5 & & 2 & 7 \\
\hline Advanced & 1 & & - & 1 \\
\hline Total & 12 & & 4 & 16 \\
\hline \multicolumn{5}{|c|}{ University Size } \\
\hline QM stage & $S$ & $M$ & $L$ & Total \\
\hline Lacking & & 1 & & 1 \\
\hline Embryonic & 2 & 4 & 1 & 7 \\
\hline Developing & 2 & 4 & 1 & 7 \\
\hline Advanced & 1 & - & - & 1 \\
\hline Total & 5 & 9 & 2 & 16 \\
\hline \multicolumn{5}{|c|}{ University Location } \\
\hline QM stage & Urban & & Periphery & Total \\
\hline Lacking & 1 & & - & 1 \\
\hline Embryonic & 1 & & 6 & 7 \\
\hline Developing & 4 & & 3 & 7 \\
\hline Advanced & 1 & & - & 1 \\
\hline Total & 7 & & 9 & 16 \\
\hline \multicolumn{5}{|c|}{ Range of Studies } \\
\hline QM stage & Monothematic & & Multi & Total \\
\hline Lacking & 1 & & - & 1 \\
\hline Embryonic & 1 & & 6 & 7 \\
\hline Developing & 2 & & 5 & 7 \\
\hline Advanced & 1 & & - & 1 \\
\hline Total & 5 & & 11 & 16 \\
\hline
\end{tabular}

From the above table, correlations between the university characteristics and quality management stage did not appear, except perhaps that peripheral universities tended to be in the embryonic stage of quality management. 
As I have already indicated, the university mission and decision-making structures were equal in all 21 universities so that there was no need to include these as control variables. The most relevant and interesting control variable appeared to be leadership (chapter 8). The leadership was related to the adoption of quality management. Moreover, leadership appeared to play an important role in the decision of taking part in the EUA-IEP (chapter 7).

Metaphorically speaking, leadership commitment reflects only the "tip of the iceberg" regarding adoption of quality management. It seems that neither the little bit of variation that I found regarding mimetic and normative pressures, nor leadership commitment could not explain why some universities did, and others did not initiate changes concerning quality management in all 21 universities.

Regarding isomorphism and adoption of quality management at the meso level, and mostly keeping in mind conclusions from chapter 7 , one surmise is that normative and mimetic pressures simultaneously came to bear on universities that did engage more extensively with quality management. An important aspect of normative isomorphism was related to EUA-IEP evaluators, who were and are perceived as carriers of the professional norms of strategic and quality management. In addition, the university leadership may have been the route of normative pressure in the link between the EUA and the university as a whole. In contrast, mimetic pressure proved to be associated with the university's location (quality management as peripheral universities' way to compete for prestige). The mimetic argument is supported by the fact that formal quality management systems were absent in all participating universities and EUA-IEP teams indicated vision (which might have included quality management as an integral part of strategy) as a missing element in most of the participating universities. In chapters 8 and 9, normative and mimetic pressures were also observed as high in all cases. Table 11-10 summarizes, by approximation, the role that the types of isomorphism played concerning adoption (or not) of quality management at the meso level in Greek higher education.

Table 11-10 Isomorphic pressures at meso level in Greek higher education system

\begin{tabular}{cccc}
\hline & Coercive & Normative & Mimetic \\
\hline Meso Level & - & ++ & ++ \\
\hline Legend: - type of pressure absent; + type of pressure present; ++ type of pressure strongly present.
\end{tabular}

The picture regarding the quality management practices of the Greek universities until 2007 could be summarized in words taken from an EUA-IEP report: "there 
were no clear quality policies at all". The EUA-IEP teams did not find "strategies for improving quality". And "there is hardly any systematic quality assessment developed in the university X or in other higher education institutions in Greece".

\subsection{Micro Level Views on Isomorphism and Quality Management}

In chapters 9 and 10, the empirical study was designed to answer the micro level (academic departments, laboratories, and academic support services) of the second part of the third research question. In order to construct the overall micro level view, the findings from these empirical studies will be summarized and discussed here under two headings: a) perspectives from department heads, "transformed" into quality management dimensions (chapter 9) and b) documented quality management (chapter 10), i.e. formal, documented quality management practices following ISO standards.

\subsubsection{Department Heads' Perceptions and Concerns on Pressure and Adoption of Quality Assurance}

In chapter 9, I employed an electronic survey in order to collect data about isomorphism and adoption of quality management from department heads in Greek universities. In this section, I concentrate on the core of the findings, which regarded the heads' own departments. (In the previous section, some data from this questionnaire with relevance to the meso level were used).

Previous research (de Boer, 2003; Ursin, 2007) has shown that department heads' perceptions are critical to the successful adoption of quality management. The questionnaire that was used in this study was largely modeled on the questionnaire instrument used by Ursin (2005, 2007). In addition, I included questions in order to collect data regarding isomorphic pressures and adoption of quality management practices (see Appendix C). This study took place in 2007 when quality assurance was being launched in Greek higher education. This survey did not directly relate to quality management but it provided insight into department heads' views on the adoption of quality assurance systems. One of the core assumptions of this study was that if academics resist or have a different understanding of quality assurance than the policy-makers or institutional leadership, introducing quality management in a university will not be effective and may even have counterproductive effects. Academics' cooperation in quality improvement is therefore crucial. Quantitative and qualitative data provided information about isomorphism and adoption of quality assurance. Responses were received from 22 department heads from 11 universities and four 
disciplines. Sixteen (out of 22) questionnaires were from respondents from universities that participated in the EUA evaluation process (chapter 7).

The 22 respondents indicated that in their own departments, four quality assurance systems were in the lacking stage of quality management, nine were in the embryonic stage, eight in the developing, and one department head was not aware of his unit's stage of development (did not know). Eleven department heads gave answers indicating concurrency between their opinion on applicability of quality assurance in higher education and the quality assurance stage of their department. Besides, one might expect that respondents would give correlated answers regarding usefulness of quality assurance systems and the benefit of quality assurance for their department. However, the actual responses to these questions were mixed. For instance, one department head who reported that the quality assurance system had not been of benefit for his/her department responded also that the usefulness of a quality assurance system was 3.8 (i.e. fairly high). Similarly, the average score of usefulness from the five department heads who reported not knowing whether a quality assurance system was or was not a benefit in the quantitative part of the survey were clearly above the midpoint of the scale, ranging from 3.3 to 3.9.

Going on to the connection of judgments with the stage of quality management in their department, eleven department heads, i.e. half the respondents, gave answers with corresponding values for the applicability of quality assurance and the current quality assurance stage of their department. The other half of respondents, however, held opposing views: low applicability but 'developed' quality management, or conversely, high applicability but only 'embryonic' quality management. Perhaps an explanation of the antithesis for the latter half of the respondents could be that these department heads gave a socially desirable answer on the applicability question. An alternative explanation could be that even if they genuinely believed that quality management was applicable to higher education institutions, they had not been able to implement it in their own department due to organizational problems (e.g. lack of support among their department's staff, or from the university leadership).

In terms of isomorphic pressures at the departmental level, department heads mostly thought that mimetic pressures (i.e. many other departments already adopted quality assurance systems) would lead them to adopt a quality assurance system. I considered pressure from the faculty within a department as a normative pressure for adoption; however, under this pressure not all department heads were supportive for a quick adoption of a quality assurance system. Coercive pressures (from legal and financial frameworks) as well as other 
normative pressures (from employers, family, and students) were perceived as weak forces when it came to the adoption of quality assurance systems. These results highlighted the dynamics, the autonomy, and the power that professors (faculty) had in Greek universities. The EUA-IEP evaluators (chapter 7) had also recognized this fact: "There is an autonomy rarely experienced in Europe when looking at the freedom of the professors in not only the content of their research and courses [but also] the basic idea of academic freedom". In the international quality assurance literature "outside" stakeholders such as employers, alumni, and family were often mentioned as drivers for adoption of quality assurance, but in Greece their influence would seem to be remarkably low. Instead, the department heads' responses strongly focused on copying (mimetic isomorphism) what other faculties or departments had already done rather than to bow to pressure from the quality assurance law and the governmental funding process (coercive pressure). As weak as it was, the normative pressure derived mostly from the departments' staff members' demands rather than from other, external sources such as professional networks.

\subsubsection{Isomorphism and Use of ISO in Laboratories and Academic Support Services}

Chapter 10, the final empirical chapter in this book, was designed to collect data about isomorphic pressures and adoption of quality management in laboratories and academic support services. These units' directors' perceptions reflected another area at the micro level in Greek higher education. Also of interest at this level, I found data that documented adoption of ISO standards as a quality management practice. This current study employed sequential qualitative data collection; first, through a survey and second, through telephone interviews (interview protocol in Appendix D). The study targeted 'positive' cases, i.e. laboratories and support units that had adopted ISO standards; I then inquired about the circumstances that had led to their adoption.

All three categories of isomorphic pressures (coercive, normative, and mimetic) were included in this study. The survey revealed that ISO standards were applied in seven Greek universities out of the 21 total. Out of the wide variety of ISO standards, ISO 9001:2000 and ISO 17025 were the most popular in engineering (mechanical, chemical, civil) laboratories. Specifically, in one university, the motivation to adopt ISO standards was supported by the university's rectorate, who strove to increase the university's competitiveness. Three cases indicated coercive pressure, in the sense that ISO certification was required by an externally funded project. The directors of the three laboratories under coercive pressure 
were negative towards this quality practice in some respects, because they believed ISO to be bureaucratic.

Most cases, 17 out of 23 (laboratories and academic support services) involved a combination of normative and coercive pressures. In a counter case of coercive (market) pressure, one interviewee pointed out that ISO certification was not used any longer, because their services were not connected anymore with market needs. Nevertheless, the director pointed out that the laboratory's staff in fact still followed ISO requirements and that they had adopted the style of this quality practice. Even if their ISO certification had expired, quality practices and a quality culture in this laboratory were still present.

Historically, the first ISO application in Greek higher education appeared in 1994, when several laboratories, library services, and a Research Committee gained ISO recognition. Some of them reregistered several times since, which indicates how long several universities have voluntarily participated in this quality management practice. In doing so, they have retained a competitive edge in ind ustrial research programs, while also earning the corresponding income to support those research departments. However, the extent to which organizational performance can effectively be improved through ISO standards remains an important issue. Whether adoption of an ISO certification is a valid indicator of high quality organizational performance across a wider swath of university operations other than science and library services needs further investigation. Besides, ISO standards will most likely remain extremely limited in their application to units experiencing very specific, market-driven coercive pressures. Nevertheless, the adoption of ISO standards is a step in the direction of measuring and evaluating production and performance in universities. Hence, it becomes a step towards adopting improvement-oriented practices across the entire organization. As already noted there is progress-although slow, as universities adopt and require quality management practices in teaching, learning, and services.

\subsubsection{Bringing together Isomorphism and Quality Management at the Micro Level in Greek Universities}

At the micro level in Greek universities, laboratories had different perspectives than academic departments and academic support services. ISO standards were more popular in engineering and medical fields. In contrast, some heads of engineering departments reported (in chapter 9) that their departments (separate from the laboratories) would never adopt quality assurance systems that were being used in the laboratories. Apparently, it is possible that a person in the 
context of a laboratory is pro-quality management, and against adoption in the department. Isomorphic pressures differed for these types of units. Department heads felt a pressure, mostly mimetic, and in some degree, coercive. In contrast, quality management practices such as ISO exist in laboratories and in academic support services because all three types of isomorphic pressures were present, as well as the leadership motivation. Table 11-11 summarizes, by approximation, the role that the types of isomorphism played at the micro level (in academic department and in laboratories and academic support services) in Greek universities concerning adoption (or not) of quality management.

Table 11-11 Isomorphic pressures at micro level in Greek Universities

\begin{tabular}{lccc}
\hline Micro level & Coercive & Normative & Mimetic \\
\hline Department & + & - & ++ \\
Lab \& ac. services & + & + & + \\
\hline Legend: - type of pressure absent; + type of pressure present; ++ type of pressure strongly present.
\end{tabular}

\subsection{Overall View Regarding Isomorphic Pressures and Adoption of Quality Management in Greek Higher Education}

The previous three sections $(11.2,11.3$, and 11.4) started the integration of the environmental scan and five empirical studies in order to respond to the third research question, about the factors that influenced stability and/or change towards quality management in Greek higher education. Those sections addressed the different organizational levels (macro, meso, and micro) in Greek higher education, addressing the second part of the third research question, which was Do the organizational factors differ at the macro level (higher education system), the meso level (individual universities) and the micro level (department, laboratory and academic support services)? Together, these answers gave detailed indications, which can now be integrated to come back to the third research question in general: Which organizational factors for stability and/or change concerning the adoption of quality management can we find empirically in Greek higher education? Starting from the neo-institutional theoretical approach, which emphasized the pressures exerted upon higher education by the organizational field, the following Table 11-12 was constructed to illustrate graphically, by approximation, the role that the types of isomorphism played at the different levels in Greek higher education concerning adoption of quality management. The table is not designed to plot exact magnitudes of the pressures; it displays approximations 
based on the available data and is useful to discern and unfold the isomorphic pressures on Greek higher education.

Looking at the table horizontally, i.e. by level of the higher education system, as it was built up in the previous sections, first of all at the macro level, it indicates that the quality assurance law (3374/2005) was adopted mainly due to mimetic pressure from other European countries (through the Bologna Process). At the meso level, changes occurred due to normative and mimetic pressures (EUA as a professional organization, examples from foreign universities etc.). At the departmental micro level, coercive (within the university) and mimetic pressures (departments in other universities) were found, although documented quality management was not detected during this study. Also at the micro level, laboratory and academic support services experienced all three isomorphic pressures (addition of market demands, in particular, to the pressures as seen on departments).

Table 11-12 Types of isomorphic pressures on different levels of the Greek higher education system

\begin{tabular}{|c|c|c|c|}
\hline & Coercive & Normative & Mimetic \\
\hline Macro & - & - & ++ \\
\hline Meso & - & ++ & ++ \\
\hline Micro: Department & + & - & ++ \\
\hline Lab \& ac. services & + & + & + \\
\hline
\end{tabular}

By reading table 11-12 vertically, looking for the impact of the types of pressure across the different levels of the higher education system, one can observe the organizational factors for stability and/or change concerning the adoption of quality management that I found empirically in Greek higher education. Coercive pressure appears as the least present type of isomorphic pressure, with normative taking second place. Mimetic pressure seems to be the dominant type of isomorphism, which was present at all levels investigated by this study. These empirical studies provided data to answer the first part of the third research question.

However, in addition to the pressures from the organizational field emphasized in neo-institutional theory, universities are complex organizations, and to that 
end, I incorporated the intra-organizational control variables: age, location, size, range of studies, mission, vision, decision-making and leadership. In terms of these university characteristics, leadership proved to play a crucial role in the adoption of quality management at the meso and micro (laboratories and academic support services) levels. At the macro level, too, though, the significant leadership role by the Minister of Education in 2005 was remarkable. While Ms Marietta Yiannakou was Minister of Education, more changes were initiated than in many previous years. Of course, a question that yet remains, for if, under the mimetic pressures from the Bologna Process, there were any other options open, it still seems that her personal involvement did make a difference. Certainly a strong leadership role is important for instituting change, as documented in the literature.

The picture regarding other organizational characteristics is less clear. Of the eight universities that participated in the EUA-IEP, none were monothematic or located in Athens. EUA-IEP participants were old, medium or large sized and multidisciplinary universities. In contrast, ISO practices were predominately adopted in units (often laboratories) within universities, located in Athens as well as in the periphery, and the universities in which these units were located were monothematic and old. Neither of the quality management practices EUA-IEP and ISO were attractive to new or small universities (although ISO certification was adopted by the academic support service in one small university). Another quality management system, the EMAS, was found in one university in the periphery.

In Greece, universities follow rules and regulations; meanwhile, the law heavily influenced daily operations under the frame-law 1268/82, up until 2006. Since then, new rules and regulations under the 2007 law are in effect. As previously mentioned, one leader (chapter 8) stated that some of the items in the category of "Process Management" were related to requirements derived from the frame-law $1268 / 82$. Although a quality management movement exists in Greek higher education, for the moment it remains small and is making painstakingly slow progress. 


\subsection{Reflections on Neo-Institutional Theory and Mixed Methods Methodology}

\subsubsection{Neo-Institutional Theory and Isomorphic Pressures}

This subsection is intended to address the fourth and final research question, which concerned whether the empirical observations and evidence coincide with the theoretical lenses, which were adopted for this study.

In the theoretical framework (repeated in Figure 11-1), three types or sources of isomorphic pressures were included, augmented with organizational characteristics. As the previous sections show, it was possible to interpret the developments in Greek higher education in these terms and they helped direct the view to relevant factors. However, it did not prove possible to identify clearly distinguishing factors of the units that did engage in quality assurance in contrast with those that did not. For instance, it was not possible to find high correlations between the presence of isomorphic pressures and the stage of development of internal quality management in universities. To a large extent, the modest result of my empirical study must be ascribed to the difficulties of empirical social scientific research in Greece. Although I tried to circumvent that problem through a multi-stage, multi-method approach, the low response rates to almost all studies I undertook, make it difficult to draw definitive conclusions that can be generalized to all Greek universities.

From a methodological point of view, the study's overall analysis demonstrated that although difficult, it is possible to isolate different isomorphic pressures in practice. I agree with Mizruchi and Fein (1999) that coercive, normative, and mimetic isomorphism are not easily distinguished empirically. Many authors lump normative and coercive pressures together in their studies. In my operationalization, I attempted to obtain sharper definitions of the two terms. This process may have included a narrowing of the theoretical concepts, but by focusing on their essential characteristics, it proved possible to investigate the two types of pressures independently. For instance, the department head's survey tool (chapter 9) was designed to investigate normative pressure through specific and clear questions (Appendix C questions 28, 29, 30). In the same survey, another question (question 31) targeted mimetic pressure. In chapter 10, using telephone interviews, I guided participants in a Socratic way to achieve clear understanding concerning coercive, normative, and mimetic pressures as discussed. Thus, also in this latter study, respondents were able to articulate clearly the types of isomorphic pressure they experienced. 
Additionally, to distinguish normative from mimetic pressure, albeit only by inference, it was helpful to pay attention to what happened after the adoption of the quality management. If follow-up activities took place in an organization, they were taken as a signal that mostly normative rather than mimetic isomorphic pressure had occurred. I reasoned that mimetic isomorphism would be correlated with symbolic adoption of organizational changes, which would not lead to serious follow-up activities.

\subsubsection{Methodological Aspects of a Mixed Methods Study}

In this subsection, I will reflect on the multi-level mixed methods design of the study. Overall, the multiple study mixed method design with both concurrent and sequential data collection proved to be appropriate; it provided a useful, analytic and sophisticated insight under difficult circumstances for an empirical study. For instance, isolating different isomorphic pressures in practice was facilitated by the use of the mixed method design.

To assess the inference quality of this study, I answer the questions related to interpretive rigor, suggested by Tashakkori and Teddlie (2003) and Teddlie and Tashakkori (2009). Greene (2007, p. 174) noted that the "knotty issue of judging the quality of inferences yielded by a study with multiple and interactive assumptions and stances remains a conceptual and procedural challenge", but their questions assisted in untangling that knot (see Table 11-13).

Table 11-13 Inference quality answers

\section{Interpretive Consistency}

1a) Do the inferences closely follow the relevant findings in terms of type, scope, and intensity?

The inferences closely follow the relevant findings

1b) Are multiple inferences made on the basis of the same findings consistent with each other?

All findings are used only once, so that this issue did not arise.

\section{Theoretical Consistency}

2) Are the inferences consistent with the theory and state of knowledge in the field?

Overall the empirical observations and evidence coincided with the theoretical lenses adopted for this study.

\section{Interpretive Agreement}

3a) Are other scholars likely to reach the same conclusions on the basis of the same results?

Yes, because I made the connections between data and conclusions explicit. 
3b) Do the inferences match participants' constructions?

In most parts of my studies, participants were not asked to reflect on my inferences. In the telephone interviews (chapter 10), it was possible without much additional explanation to reach a match between respondents' understanding of reality and my study's concept of isomorphic pressures. Besides, the latest policy changes and laws supported the answers given by the Greek university leaders.

\section{Interpretive Distinctiveness}

4) Is each inference distinctively more credible/plausible than other possible conclusions that might be made on the basis of the same results?

This seems plausible; for instance, I also considered the possibility to use the resource dependency approach in combination with the neo-institutional theory. The current theoretical "lenses" are more parsimonious than a theoretical approach with resource dependency theory; of the control variables, only leadership proved consistently relevant.

\section{Integrative efficacy (mixed and multiple methods)}

5a) Do the meta-inferences adequately incorporate the inferences that are made in each strand of the study?

Yes (see overall results)

5b) If there are credible inconsistencies between the inferences made within/across strands, are the theoretical explanations for these inconsistencies explored, and possible explanations offered?

The overall findings demonstrate that there were no major inconsistencies across strands, except for the-theoretically easily accommodated-different weights of the different types of isomorphic pressures at different levels (macro, meso, and micro).

\section{Interpretive Correspondence}

6a) Do the inferences correspond to the stated purposes/questions of the study? Do the inferences made in each strand address the purposes of the study in that strand?

Yes (overall inference of the study)

6b) Do the meta-inferences meet the stated need for utilizing a mixed methods design? (i.e., is the stated purpose for using mixed methods met?)

Yes, as study of pressures for and against quality assessment in a multi-level, multi-actor system (such as a higher education system) using neo-institutional theory is a clear fit for MM design. The current study demonstrates that the same conclusion would not have been attainable with a single method.

Since the samples in the empirical studies in chapters 8, 9, and 10 represented participants from a limited number of universities, and because data were gathered in a particular period (2005-2007), the extent to which the present findings can be generalized to other universities or other periods was not clear. This fact in mixed methods terminology refers to the study's inference 
transferability (Tashakkori \& Teddlie, 2003, p. 710). Some phenomena are difficult to transfer, and difficult to generalize outside of the study's contextual conditions. Greene (2007, p. 173) proposed that "the quality of inferences in mixed methods social inquiry be judged by the ways in which and the extent to which the study contributed to better understanding" rather than by generalization. I think I succeeded in increasing the insight into a complex organizational phenomenon under difficult research circumstances through conducting a mixed methods analysis combining qualitative and quantitative approaches and quantified qualitative data at three different system levels. Overall, this amalgamation provided a fairly coherent explanation of the observed phenomenon (quality management in higher education) and its relationships with the three types of isomorphic pressure (sections, 11.5 and 11.6).

A noteworthy characteristic of this study is that I employed five empirical studies and an environmental scanning. It was not until after all these studies were completed that the overall results became clear. For example, as noted (chapter 10) during the final empirical study, I discovered a quality management practice at the meso level, although the study was designed to investigate the micro level. From a methodological point of view, this study is important because it merges multiple approaches and practices and provides a flexible strategy for research, which should benefit future researchers. Finally, this study contributes to the call in the Journal of Mixed Methods Research (JMMR) Creswell \& Tashakkori, 2007, p. 107), for studies using a multi-level mixed design to test and examine the concept of organizational theory as applied to organizational change practices.

\subsection{Future Avenues of Quality Management Research in Greek Higher Education}

This study illustrates the complexity of the adoption of quality management and the introduction and implementation of policy related to quality. Leadership, culture, politics and ideology, ceremonial essence, training, and resources (budget and human) all provide future avenues for quality management research. Not surprisingly, the areas are numerous because the field of quality management in Greek higher education is relatively new and Greek higher education institutions will need to change much before there will be widespread adoption of academic quality management practices. Therefore, I moved forward in an attempt to identify practices ('best', 'good' or simply 'effective') from Greece, but also from foreign quality management systems, which might assist other units in Greek higher education to adopt such practices in the years ahead. The knowledge and experience that I gained from the study in conjunction with previous research 
projects, all seemed essential for this new journey. Some of the areas that were identified for further exploration are included the following paragraphs.

For expansion of the empirical base, the 16 technological colleges (TEIs) need to be studied ${ }^{40}$ regarding their perceptions of quality management. A comparison between TEIs and universities might be beneficial to study the influence of different isomorphic pressures and different organizational characteristics. Considering the large data potential, that sort of comparison could provide a vast array of topics and avenues for additional research.

Another option starts from the realization that policy implementation is a multifaceted phenomenon. In light of this complexity, the implementation of the quality assurance policy that finally started to become operative in Greek higher education after 2009 and its effect in changing behavior within higher education institutions at different levels (meso and micro) offers itself as an avenue of research.

Another area that needs additional attention relates to the development of institutional research units. Chatzipantelis and Papadimitriou (2010) suggested development of such research units, stating that it is a necessity for the Greek universities, not only to support decision-making in universities but also to provide up-to-date and accurate data in case those Greek universities are concerned about their ranking and benchmarking with each other. For research, such units might prove useful as sources for interactive surveys. More directly related with university practices, such units might discuss advantages and challenges associated with the development of additional research units, or collectively solve problem issues, and contribute to the general body of knowledge about higher education management improvement. Last but not least, research on how students' learning outcomes, which are leading recent developments in the Bologna Process, integrate into the quality management of the university merits further exploration.

\footnotetext{
40 Recently, a study by Tsinidou et al. (2010) aimed to identify the quality determinants for education services provided by TEIs in Greece and to measure their relative importance from the students' points of view. The authors distributed 300 questioners to undergraduate students of all departments in the School of Business and Economics in one institution namely Technological Educational Institute of Larissa.
} 


\subsection{Navigating the Archipelagos of Greek Higher Education: Recent and Future Challenges and Changes in Greek Higher Education}

Finally, this section attempts to discuss some issues of the "meta-quality assurance law" period (2006-2010) and offer a better understanding of the challenges and changes in Greek higher education. This section incorporates multiple views of the current process as I attempt to navigate through the archipelagos of Greek higher education under the turbulences of ever-changing weather conditions. I believe this navigational metaphor matches well not only the Greek topography but also its higher education challenges.

Katsikas, Papazoglou, and Tsakloglou (2008), discussed how the problems are many; generally, they observed the absence of a quality assurance culture, resistance from several groups, and a type of insecurity with a fear of something new and unknown. They stated that the unknown status of quality assurance resulted from "ignorance for the relative procedures or and disinformation (black propaganda)". The authors also underscored the lack of confidence on the side of the academic departments, because the Ministry of Education did not make clear statements about the use of the departments' evaluations, which made up an important part of the new law. Kladis (2008) also noted that there is a negative attitude in Greece against quality assurance (among academics, among students, and even among rectors). He mentioned two reasons for this attitude: "The general Greek mentality against any type of control and the lack of trust between the State and the HEIs".

As discussed earlier, a "window of opportunity" opened in 2005 and the Ministry of Education introduced the quality assurance law; however, until now, it seems that the wind of change is not blowing from that window to encourage formalized, improvement-oriented quality management in the Greek archipelagos. In contrast, at the macro level, in 2007, the Ministry of Education launched transformation law 3549/2007, which introduced many changes, of which the most relevant concerned decision-making and leadership structures and processes, as well as introduction of strategic planning. On the surface, it would appear that this law attempted to focus Greek higher education on performance improvement and effectiveness. Thus, according to Kyriazis and Asderaki (2008, p. 43): “through the 'Four-year Development-Academic Planning' established HEIs will be able to handle their finance more effectively and to plan ahead their teaching and research activity according to their mission, their special goals and profile". 
From academic year 2009-2010, the new Ministry of Education requires all higher education institutions to submit a self-assessment report and to invite external evaluators for review. Otherwise, departments will face sanctions in funding and human resources. Surfing the Greek higher education institutions' websites since then, one can observe changes in universities' attitudes. Departments published self-evaluation reports on their websites, and there are now rectors' messages advertising how their universities meet $21^{\text {st }}$ century expectations regarding quality assurance. It seems that coercive pressure motivated universities to comply at least symbolically.

In this archipelago, quality management is seen mainly in terms of laws and regulations, and it seems that the Ministry of Education introduced regulation to make universities develop formalized management practices. This coercive pressure underlines legitimacy issues rather than quality management practices geared towards actual quality improvement. The adoption of quality management systems for improvement purposes, according to my study's results, requires the 'tripod' of pressures: not just coercive, but also normative and mimetic isomorphic pressures. To change academic quality culture in such an environment, a lot of work is needed. Therefore, it seems that the major challenge for the Ministry of Education is to find an appropriate strategy to change academic quality culture. Probably, this puzzle could begin completion if the other legs of the tripod (normative and the mimetic isomorphism) were developed as well as the legal (coercive) one.

There may be a productive connection between coercive and normative pressures: the transformation law obliges higher education institutions to fill the new position of higher education secretary (institutional manager). Kyriazis and Asderaki (2008, p. 48) wrote that "this position has been introduced for the better management of administrative and financial matters". In neo-institutional terminology this new position, bringing a set of professional managers into highlevel positions in all Greek higher education institutions, could create normative pressure in the future. However, until 2010, although the majority of the Greek universities announced this position, few have yet appointed a secretary.

I can see another route of normative isomorphism that is in place since 2007. The Hellenic Quality Assurance Agency (ADIP) could be regarded as a professional body exerting normative pressure. For example, one department head (quoted in chapter 9) mentioned: "Not being a specialist in the field I would be very happy to be guided by the specialists". More work needs to be done, as previously discussed, because as another department head (chapter 9) noted: “The Greek academic environment is a very difficult one for implementation of a quality 
assurance system. The legal framework is present but in my opinion, it shall never be implemented. No one really wants it and if ever implemented it shall be only paper". Parallel observations were found in some EUA-IEP reports (chapter 7), which stated, "A central evaluation committee established under the Law of 1992 did not receive the cooperation of the academic community. In 1995, the National Education Council was created, which aimed to evaluate institutional quality, but five years later it has yet to function".

At the meso level, one could think of establishment of a unit for institutional research in each university. In countries like Greece, where higher education studies are quite underdeveloped, institutional research would importantly contribute to developing skills in the higher education field. To achieve desired results, quality management must become a routine way of work in higher education institutions. Therefore, institutional research units should also focus on training at different levels in a university, so that administrators and faculty can begin their journey to quality with tools that create confidence. Horine and Hailey (1995) reported the key variables that affect the successful quality management implementation in higher education institutions: organizational culture, senior leadership commitment, faculty support, implementation time, and training. Additionally, Papadimitriou et al. (2008) stated that many Greek department representatives saw training as "the most important requirement in order to adopt ... [a] quality assurance system".

An issue that needs attention during training is resistance from academia, since any unknown (change) is usually met at first with resistance in that it represents stepping out of one's comfort zone, it creates fear about the unknown, the unproven, and it involves risk taking. Learning from others' experiences may be the best way to adopt best practices (Dill, 1999). Such mimicry is one missing piece in the Greek quality puzzle. Papadimitriou and Westerheijden (2009) noted that "perhaps incentives could be provided that reward the first adopters and implementation of quality management".

Moving on to the mimetic part of the tripod, regarding the Bologna Process in the 2008 National Report, the following statement appeared about challenges in higher education: "The main issue ... is not only the development of ameliorative measures by institutions in cooperation with the Ministry but also the development of a quality culture that will apply to all HE" (YPEPTH, 2008, p. 43). Indeed, in July 2010, the Hellenic Quality Assurance Agency's (ADIP) annual report $^{41}$ wrote that just over $1 / 3$ (105 out of 287) of the academic departments had

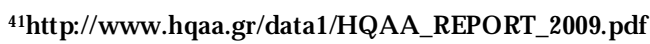


completed their internal evaluation and 31 were in process. Concerning the external evaluation processes the number of academic departments that received comments from external evaluators remained very low (eleven) until November 2010. ADIP reported that "it is interesting that the distributions of the internal evaluation reports that till now have been submitted by academic departments are not uniform" (ADIP, 2010, p. 15). Papadimitriou and Ursin (2010) reported that "in one large university, in Greece, from its 42 academic departments only one department orally mentioned that it would not participate in an evaluation process. [...] There were 17 academic departments that [...] were in process of submitting their self-assessment reports by the end of 2010. [...] Moreover, the entire university submitted the university's self-report, as requested by the ADIP, and two departments invited external evaluators". The 105 internal evaluation reports mentioned by ADIP came from 11 out of 24 universities (ADIP 2010, p. 26). In this book, I covered 21 universities, and from ADIP's report I read that after 5 years, the academic departments that belong to 7 out of 21 universities that I studied in this book have not submitted any reports. It would seem, then, that unless coercive pressure is applied and until this evaluation process is made obligatory by the Ministry of Education, there will be little forward movement. The current evidence stands as previously quoted from chapter 9, "...if ever implemented it shall be only paper".

In this archipelago, I also see new mimetic pressure upon universities, which will probably motivate them to adopt quality management practices. The new twist this time comes from ranking schemes. Universities, in Greece and elsewhere, which want to perform better in ranking, need to adopt sophisticated managerial practices not for window dressing, but for performance improvement. This twist will include the requirement that they must adopt quality management. Once big universities have adopted quality management practices by mimicking foreign institutions more successful in rankings, then in the following years their examples will be regarded as "local quality practices" for mimicking in smaller universities.

All of the pressures for change become even more urgent when one considers Greece's current economic crisis and the limited resources that are now available from state funding. Given the economic constraints of the current crisis, survival of the whole university might very well be contingent upon the entire university's adoption of managerial practices to cut costs and provide education efficiently to students. It seems that coercive pressures (economic crisis, cutting costs and associated pressures from leadership) may lead to a more normative influence that drives the meso and micro levels towards adoption of quality management and quality assurance to achieve improvements in these difficult circumstances. 
The leadership of each university must become inspiring to ensure successful navigation of each of the universities during the current, very turbulent, difficult, and unpredictable weather conditions.

Implementation of quality management needs leaders who remain in their position for more than a few years, which until now seldom happens in Greek higher education.

Against this background, the ultimate goal of this research is to assist higher education institutions to fulfil their role in society. This author refuses to be limited to politics as the only type of argument in the discussion about quality in higher education. This author regards higher education as a public good for all citizens. Yet the quality assurance law and other higher education regulations in Greece are related to party politics and ideology, sometimes leading to conflicts fought out through violent student demonstrations. One department head who perceived the quality assurance system as a disadvantage for his department (chapter 8) explicitly mentioned politics: “The Greek academic environment is not very suitable due to the power of the student bodies and because of their attachment to political parties". My analysis intended to show that quality management for improvement is a possibility, that it is used in foreign as well as some parts of Greek higher education institutions, and that it might overcome party politics.

As stated in the introductory chapter, the goal of this dissertation was to establish a framework for future analysis of quality management practices as well as identifying potential change factors. The framework generated to perform this research may be applicable to a broad range of higher education units, and to my knowledge, represents the most comprehensive effort to date of analyzing adoption of quality management in Greek higher education. However, this research is the beginning of what is likely to be a long process of thoroughly analyzing how, why, and what higher education institutions can do by way of quality management on their paths toward excellence. The findings from this dissertation are beginning to bridge the information gap on quality management in Greek higher education. My belief is that Greek higher education institutions would best support their public good role by showing society that they are on a path towards excellence; I wrote this dissertation to assist and to awaken academia to the pursuit of this difficult, yet worthwhile process. 


\section{Nederlandstalige samenvatting}

Het raadsel van kwaliteit in het Griekse hoger onderwijs: Mixed-methods studies naar de invoering van kwaliteitsmanagement

Deze studie gaat over verandering en stabiliteit in het Griekse hoger onderwijs met betrekking tot invoering van kwaliteitszorg en kwaliteitsmanagement. De studie wortelt in organisatiestudies en managementstudies in het hoger onderwijs; introductie van kwaliteitszorg wordt gezien als een voorbeeld van organisatorische verandering.

Tenminste tot 2007 bevond kwaliteitszorg in het Griekse hoger onderwijs zich in een vroeg en omstreden stadium. Doel van de studie was de invoering, of nietinvoering, van kwaliteitszorg in universiteiten te bestuderen als een uitkomst van organisatorische veranderingsprocessen. De probleemstelling van het onderzoek was de identificatie van de relaties tussen organisatorische factoren die stabiliteit en/of verandering stimuleren van de introductie (of non-introductie) van kwaliteitszorg in Griekse universiteiten. De algemene probleemstelling werd onderverdeeld in drie onderzoeksvragen:

1. Wat verstaan we onder kwaliteitsmanagement in hogeronderwijsinstellingen?

Deze vraag werd beantwoord (in hoofdstuk 2) door middel van een literatuuroverzicht van kwaliteitsmanagement, met aandacht voor de instellingsevaluaties door de EUA (EUA-IEP), de Malcolm Baldrige criteria voor excellentie (MB), en kwaliteitsbenaderingen via ISO-standaarden. Bovendien illustreerde ik dit met enkele voorbeelden van kwaliteitsmanagement in hogeronderwijsinstellingen in het buitenland.

2. Welke organisatorische factoren kunnen worden onderscheiden met betrekking tot stabiliteit en/of verandering met betrekking tot de invoering van kwaliteitsmanagement in de literatuur over organisaties en hogeronderwijsinstellingen?

De opensysteemtheorie en neo-institutionele benaderingen gaven mij ingangen om de verschijnselen te benaderen (hoofdstuk 3). Met name het concept van isomorfie was zeer nuttig. Volgens neo-institutionele benaderingen opereren organisaties, zoals universiteiten, in een omgeving bestaande uit regels, vereisten, 
concepties, veronderstellingen, overtuigingen en procedures over wat gepast of acceptabel is. Met name onderscheidt het neo-institutionalisme (DiMaggio \& Powell, 1983) drie mechanismen waardoor isomorfie optreedt: dwang door wettelijke of politieke druk en die te maken heeft met legitimiteit, mimetische druk (kopieerdruk) resulterend uit standaard reacties op onzekerheid, en normatieve druk die te maken heeft met professionalisering. Deze drie factoren werden aangemerkt als de belangrijkste onafhankelijke variabelen.

Daarnaast concipieerde ik hogeronderwijsinstellingen als complexe organisaties bestaande uit diverse lagen en met bijzondere kenmerken. Een aantal organisatorische kenmerken werd daarom toegevoegd als controlevariabelen met betrekking tot de introductie van kwaliteitsmanagement. In Griekenland zijn de missie en structuren van de universiteiten in de wet vastgelegd; deze organisatorische kenmerken waren daarom geen variabelen in mijn studies. De overblijvende organisatorische kenmerken waren: leiderschap, visie, leeftijd, grootte, locatie en breedte van studieaanbod (hoofdstuk 3).

Een en ander resulteerde in het volgende conceptuele raamwerk (figuur 1). De variabelen werden in hoofdstuk 4 geoperationaliseerd.

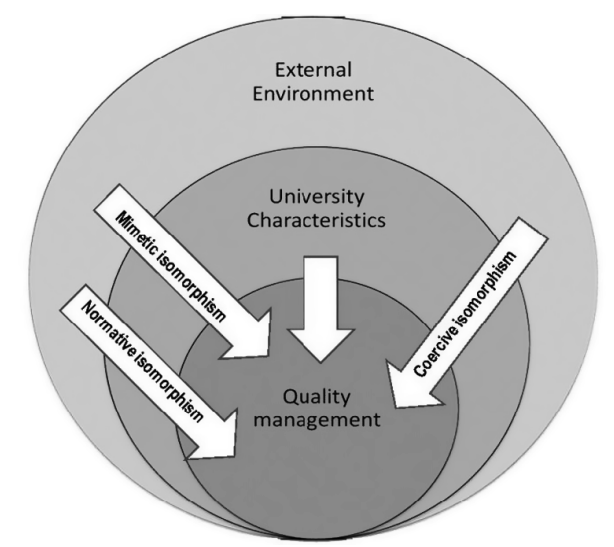

Figuur 1 Conceptueel raamwerk van de studie

De derde onderzoekvraag betrof de empirische studies en bestond uit twee delen. Het eerste deel was de hoofdvraag; het tweede deel specificeerde haar naar de diverse systeemniveaus.

3. Welke organisatorische factoren kunnen empirisch worden onderscheiden met betrekking tot stabiliteit en/of verandering met betrekking tot de invoering van kwaliteitsmanagement in het Griekse 
hoger onderwijs?Verschillen deze organisatorische factoren op de systeemniveaus macro (hele hogeronderwijssysteem), meso (instelling) en micro (vakgroep, laboratorium, ondersteunende dienst)?

Voor de empirische studies was de keuze van Griekenland als locatie van de studie een complicatie, niet alleen omdat de invoering van kwaliteitszorg op dat moment (2005-2007) onderwerp van een verhit debat was, maar ook omdat Griekenland bekend staat wegens de slechte respons op elk soort empirische sociaalwetenschappelijke studie. Om deze redenen (plus andere, genoemd in hoofdstuk 4), werd gekozen voor een onderzoeksdesign met mixed-methoden en op verschillende systeemniveaus (figuur 2).

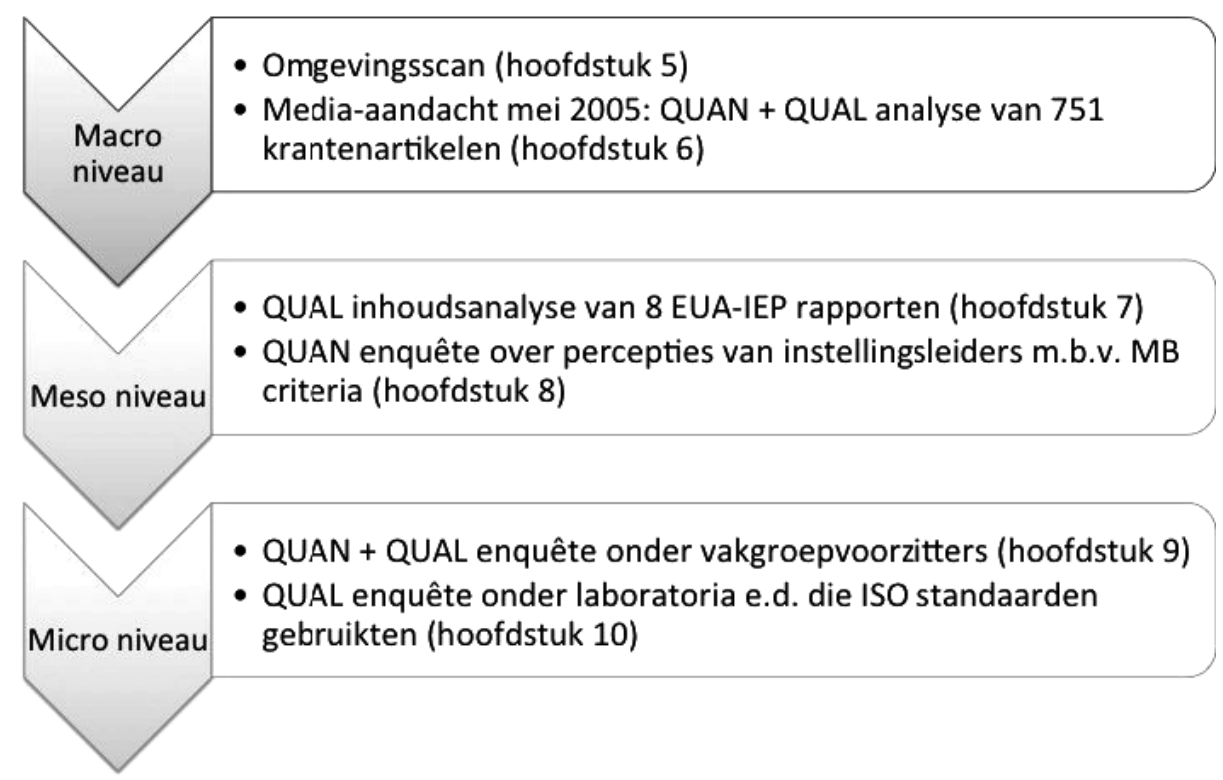

Figuur 2 Overzicht van empirische studies 
De laatste vraag in deze studie betreft hoe de empirische bevindingen de theotretische concepties valideren dan wel falsificeren:

4. Komt het empirisch materiaal betreffende de invoering van kwaliteitsmangagement in Griekse universiteiten overeen met de theoreitsiche benaderingen die in deze studie zijn geformuleerd?

Als voorbereiding op de empirische studies werd een omgevingsscan gemaakt van het Griekse hoger onderwijs rond 2005, over factoren van regulering en politiek, economie, (management) technologie, de Europese dimensie en socioculturele factoren. In hoofdstuk 6 bracht ik het verhitte debat voor het voetlicht door een inhoudsanalyse van hoe de pers kwaliteitszorg in het hoger onderwijs presenteerde. De periode van studie was mei 2005, toen de vervolgconferentie van het Bolognaproces plaatsvond in Bergen. De kranten brachten stukken die gemengd van toon waren (zowel positieve als negatieve artikelen) en die vooral normatieve isomorfische druk betekenden. Niettemin beargumenteerde ik dat de dominante druk op het Ministerie van Onderwijs mimetisch was, namelijk de collegiale druk vanuit de Stocktaking in het Bolognaproces, met zijn eenvoudig te begrijpen 'stoplicht'-scorecard die er voor Griekenland in de conceptversie nogal rood uitzag. Die druk creëerde een 'opening' voor de minister om in mei 2005 een wet op kwaliteitszorg bij het parlement in te dienen, waarna de scorecard voor Griekenland een stuk groener werd.

In hoofdstuk 7 was de vraag vooral onder welke omstandigheden universiteiten vrijwillig een evaluatie zouden aangaan; het Institutionele Evaluatie-Programma van de Europese universiteitenvereniging EUA (EUA-IEP) leverde het materiaal. Acht van de 21 universiteiten in Griekenland hadden daaraan meegedaan. Formele, gecentraliseerde of ook maar enigszins complex kwaliteitsmanagement troffen de evaluatoren niet aan in de instellingen die hen hadden uitgenodigd. Wel was er in de meeste rapporten iets terug te vinden over activiteiten die voor kwaliteitszorg relevant waren, meestal in diverse vakgroepen-wat de autonome positie van vakgroepen in het Griekse hoger onderwijs onderstreepte. Normatieve isomorfische druk werd gevonden in het feit dat de EUA-IEP alleen kon worden uitgenodigd via het professionele netwerk van rectoren dat de EUA is. Een mimetische factor werd gesuggereerd doordat alleen universiteiten uit de periferie van het land aan de EUA-IEP hadden meegedaan, niet de meer prestigieuze uit de urbane regio's Athene en Thessaloniki. De belangrijke rol van rectoren in de EUA-IEP suggereerde ook dat leiderschap een belangrijke organisatorische variabele was (naast locatie, wat zoals gezegd met mimesis samenhing). 
Hoofdstuk 8 gaf een directere benadering van de veranderingen, via een enquête onder instellingsleiders (rectoren en vice-rectoren) gebaseerd op de internationaal bekende Malcolm Baldrige kwaliteitscriteria. Deze criteria waren ontworpen in de VS om organisaties op het pad te zetten van continue kwaliteitsverbetering. De MB criteria in hun versie voor het hoger onderwijs zijn verdeeld in zeven gebieden: 1. Leiderschap, 2. Strategische planning, 3. Focus op studenten, stakeholders en de markt, 4. Meten, analyseren en kennismanagement, 5. Focus op academische en ondersteunende staf, 6. Procesmanagement en 7. Prestaties en resultaten van de organisatie. Een analyse van de diverse onderdelen kan leiders helpen sterke punten te herkennen en prioriteiten te leggen voor verbeteringen.

In mijn enquête vroeg ik de universiteitsleiders naar hun percepties van de noodzaak van management en naar de mate van toepassing van kwaliteitsmanagement in de zeven gebieden. Daarnaast stelde ik vragen over de isomorfische omgevingsdruk die zij ervoeren. Respons betrof negen universiteiten. In vrijwel alle negen gevallen waren de normatieve en mimetische isomorfische druk hoog. (Bij gebrek aan een wet op kwaliteitszorg-die was immers alleen nog maar ingediend bij het parlement-en van kwaliteitsfactoren in de financiering van de universiteiten was er geen sprake van coërcie.) Ondanks die gelijkvormige situatie, was de mate van invoering van kwaliteitsmanagement verschillend. Geen van de respondenten vond, in antwoord op één overkoepelende vraag, dat zijn universiteit een kwaliteitsmanagementsysteem had. Uit de antwoorden op de zeven MB-gebieden bleek echter dat er verspreid wel kwaliteitszorgactiviteiten te vinden waren. Die antwoorden werden samengevat tot een index van het bereikte stadium van kwaliteitsmanagement, van Afwezig(A), via Embryonaal (E) en Ontwikkelend (O) tot Gevorderd (G). Samen met gegevens verkregen van vakgroepvoorzitters (als bijproduct van de studie van hoofdstuk 9, die hieronder besproken wordt) leidt dit tot de volgende inschatting van de stand van zaken (tabel 1).

Tabel 1 Stadium van kwaliteitsmanagement per universiteit

\begin{tabular}{lc}
\hline QM stadium & Aantal \\
\hline Afwezig & 1 \\
Embryonaal & 7 \\
Ontwikkelend & 7 \\
Gevorderd & 1 \\
\hline Totaal & $\mathbf{1 6}$ \\
\hline
\end{tabular}

De antwoorden per MB-categorie vertoonden een opvallend patroon: de meeste implementatie van kwaliteitszorg vond plaats in categorie 6, Procesmanagement. 
Een van de respondenten gaf aan dat dit te maken had met eisen in de nog vigerende wet op het hoger onderwijs uit 1982 (1268/82). In met name de categorie Strategische planning was de score veel lager. Wilden universiteitsleiders liever controle over professoren krijgen dan zelf gecontroleerd worden? De controlevariabelen leeftijd van de instelling, grootte, locatie en breedte van studieaanbod leverden geen correlaties op. Naar aanleiding van de ervaringen in de eerste studies gaf ik de variabele 'leiderschap' speciale aandacht. Uit de MB-categorieën 1, 2 en 3 destilleerde ik een 'leiderschapstriade', die goed met de index van kwaliteitsmanagement correleerde.

De overige twee studies betroffen het microniveau. De ene, in hoofdstuk 9 beschreven, was een online enquête onder vakgroepvoorzitters, de andere bestudeerde model-gevallen (althans in vergelijking met andere gevallen in Griekenland) van kwaliteitsmanagement met gebruik van ISO-standaarden.

De studie in hoofdstuk 9, uitgevoerd in 2006, was een schriftelijke enquête onder vakgroepvoorzitters naar hun begrip van kwaliteitsmanagement, deels naar voorbeeld van een enquête uit Finland (Ursin, 2005, 2007). Daaraan voegde ik vragen toe over de isomorfische druk en het stadium van kwaliteitsmanagement. Van de 266 vakgroepvoorzitters, die allen waren aangeschreven, antwoordden er 22 , vanuit 11 verschillende universiteiten, die slechts deels overlapten met degene waarover in hoofdstuk 8 gegevens waren verkregen.

Voor de helft van de vakgroepvoorzitters gold dat positieve overtuigingen over de toepasselijkheid van kwaliteitszorg in het hoger onderwijs samengingen met een verder ontwikkeld stadium van kwaliteitsmanagement in hun vakgroep en negatieve met een laag stadium. In de andere helft was er niet zo een samenhang, misschien door sociaal wenselijke antwoorden ten aanzien van de toepasselijkheid terwijl zij geen kwaliteitsmanagement in hun vakgroep wensten, misschien door organisatorische problemen in hun vakgroep (tegenstand?) bij de invoering van kwaliteitsmanagement die zij wel wensten.

De respondenten gaven aan dat mimetische druk (dat wil zeggen het voorbeeld van vele andere vakgroepen in hun discipline) hun tot invoering van kwaliteitsmanagement in hun vakgroep zou leiden, zei het vaak niet met grote urgentie. En voor zover normatieve druk een rol speelde, was het die van de overige leden van de vakgroep, niet die van 'buiten' zoals bij voorbeeld professionele netwerken.

Deze studie bevatte ook enkele vragen over de perceptie van de vakgroepvoorzitters ten aanzien van isomorfische druk en het stadium van 
kwaliteitsmanagement in hun universiteit als geheel (mesoniveau). De respondenten gaven aan dat zij druk ervoeren om kwaliteitszorg in te voeren in hun universiteiten, meest mimetische druk, voor een deel dwang (tijdens deze enquête, in 2007, was de wet op de kwaliteitszorg officieel van kracht), en minder normatieve. De mimetische druk was hoger, volgens twee nonparametrische tests, op specialistische universiteiten dan op die met een breed studieaanbod. Wat het stadium van kwaliteitsmanagement aangaat, waren in vier van de vijf universiteiten met meerdere respondenten de vakgroepvoorzitters het niet met elkaar eens; zulke gegevens moeten dus terughoudend bezien worden. Voor een aantal universiteiten was de perceptie van vakgroepvoorzitters echter de enig beschikbare indicatie van het stadium van kwaliteitsmanagement van de gehele instelling (verwerkt in tabel 1).

De laatste empirische studie ging over de identificatie van eenheden (laboratoria en ondersteunende eenheden, met name bibliotheken) die kwaliteitsmanagement onder ISO-standaarden hadden ingevoerd, en de isomorfische druk waaraan deze eenheden bloot stonden. De leiders van deze eenheden werden daarop geïnterviewd. 23 Eenheden met ISO-standaarden werden gevonden in zeven van de 21 universiteiten. In laboratoria in technische disciplines ging het vooral om ISO 900:2000 en ISO 17025; in één universiteit was ISO-certificatie instellingsbeleid met het oog op de concurrentiepositie van de universiteit. Drie andere gevallen ervoeren dwang om ISO-standaarden in te voeren via voorwaarden bij extern gefinancierde projecten; de directeuren van deze drie laboratoria stonden in sommige opzichten negatief ten opzichte van de ISOpraktijken, die zij bureaucratisch vonden. In 17 van de 23 gevallen (laboratoria en ondersteunende diensten) speelde de combinatie van normatieve druk en dwang een rol. In een geval werd aangegeven dat de ISO-certificatie niet was vernieuwd, omdat er geen noodzaak vanuit de markt voor was, maar dat de processen in feite nog wel erop waren afgestemd, als bewijs van kwaliteitspraktijken en kwaliteitscultuur.

De resultaten van al deze studies werden samengebracht om een totaaloverzicht over de stand van zaken met betrekking tot isomorfische druk en de invloed ervan op invoering van kwaliteitszorg en kwaliteitsmanagement te krijgen (zie tabel 2). De tabel horizontaal beschouwend, per systeemniveau, laat ze zien dat de wet op de kwaliteitszorg (3374/2005) vooral voorwaarts werd geholpen door mimetische druk vanuit het Bolognaproces. Op het mesoniveau speelden normatieve en mimetische druk een rol (EUA als professionele organisatie, voorbeelden van buitenlandse universiteiten enz.). Op het microniveau werden dwang (binnen de universiteit) en mimetische druk (andere vakgroepen) aangetroffen, al werd geen in documenten vastgelegde kwaliteitsmanagement 
gevonden. Eveneens op het microniveau, ervoeren laboratoria en ondersteunende diensten alle drie vormen van isomorfische druk (met de dwang van de markt als bijzonderheid ten opzichte van vakgroepen).

Tabel 2 Typen isomorfische druk op de drie niveaus

\begin{tabular}{lccc} 
& Dwang & Normatief & Mimetisch \\
\hline Macro & - & - & ++ \\
Meso & - & + & + \\
Micro: Vakgroep & + & - & + \\
Lab \& diensten & + & + & + \\
Legenda: - druk afwezig; + druk aanwezig; ++ druk in hoge mate aanwezig
\end{tabular}

Bekijken we tabel 2 verticaal, dan was dwang het minst aanwezig, gevolgd door normatieve druk. Mimetische druk werd het meest gevonden, op alle systeemniveaus.

In termen van de andere organisatorische factoren, speelde vooral leiderschap een cruciale rol in de invoering van kwaliteitsmanagement op de meso- en microniveaus. Maar ook op macroniveau was de leiderschapsrol van Marietta Yiannakou, de Minister van Onderwijs in 2005, opmerkelijk.

Het beeld is diffuser waar het de andere organisatorische kenmerken betreft. Locatie en breedte van studieaanbod leken een rol te spelen voor de deelname aan de EUA-IEP. Noch de EUA-IEP, noch ISO-standaarden waren aantrekkelijk voor kleine en nieuwe instellingen (met één uitzondering voor wat betreft ISOstandaarden).

Een 'kwaliteitsbeweging' lijkt al met al wel te bestaan in het Griekse hoger onderwijs, maar vooralsnog is ze klein en is de vooruitgang bijzonder traag.

Wat betekenen de empirische resultaten voor de gebruikte theorieën? Het bleek mogelijk om meer licht te werpen op de ontwikkelingen en dat is een pluspunt. Het bleek echter niet mogelijk om duidelijk onderscheid te vinden tussen instellingen waar de invoering van kwaliteitsmanagement verder was gevorderd van andere universiteiten.

Voor een deel waren de bescheiden conclusies toe te schrijven aan de moeilijkheid om in Griekenland empirisch onderzoek te doen. Hoewel ik 
probeerde dat probleem te omzeilen door een multi-methoden onderzoeksontwerp op meerdere systeemniveaus, verhinderden de lage responspercentages in de meeste van mijn studies het generaliseren van mijn bevindingen naar het gehele Griekse hoger onderwijs.

Methodologisch interessant is dat ik erin slaagde om dwang, normatieve en mimetische isomorfische druk empirisch te onderscheiden, hoewel bij voorbeeld Mizruchi en Fein (1999) de moeilijkheid daarvan hadden onderstreept en de meeste onderzoekers slechts een of twee typen isomorfische druk onderscheiden. Dat moge een inperking van de theoretische concepten nodig hebben gemaakt, maar het bleek mogelijk om ze apart te operationaliseren, door op essentiële elementen te concentreren.

Vrij uitgebreid ben ik ingegaan op de multi-niveau, mixed-methods methodologie. Deze aanpak bleek onder de moeilijke omstandigheden nuttig om goed inzicht te krijgen; bij voorbeeld het scheiden van normatieve en mimetische druk werd erdoor vergemakkelijkt. Aan het einde van mijn werk heb ik de kwaliteit van mijn methodologie beoordeeld met behulp van een set vragen over de interpretatieve striktheid, afkomstig van Tashakkori en Teddlie (2003) en Teddlie en Tashakkori (2009) (tabel 11-13). Ze leidden onder andere tot de conclusie dat de consistentie van mijn interpretaties onderling, en in relatie met de theoretische uitgangspunten in orde is, dat mijn interpretaties via neoinstitutionele theorie en organisatorische kenmerken vrij doeltreffend en efficiënt is, en dat de doelstellingen van mijn studie ermee bereikt konden worden. De interpretatieve overeenstemming met andere onderzoekers (zouden andere onderzoekers met dezelfde gegevens tot dezelfde conclusies komen?) acht ik ook hoog. In de meeste studies heb ik geen pogingen gedaan overeenstemming te verkrijgen met de participanten in het onderzoek, maar er zijn indicaties dat zulke overeenstemming gemakkelijk bereikt kon worden (hoofdstuk 10) en recente beleidswijzigingen lijken de antwoorden van universiteitsleiders op mijn enquêtes ook te bevestigen.

In de slotparagrafen van mijn boek ging ik in op de recente beleidswijzigingen in Griekenlad. Sinds 2009 eiste het ministerie dat de hogeronderwijsinstellingen zelfevaluaties gingen uitvoeren en externe evaluaties zouden uitnodigen. In 2009/2010 evaluaties begon inderdaad de publicatie van zulke rapporten; de eindelijk uitgeoefende dwang leek ten minste tot symbolische invoering van kwaliteitszorg te leiden. Om tot meer diepgaande verandering te komen, zou volgens mijn onderzoek de complete 'drieslag' van dwang, normatieve en mimetische druk in stelling moeten worden gebracht. De kwaliteitsagentuur 
ADIP zou hierin een rol kunnen spelen, door training en informatie (normatieve druk); rankings zouden een element van mimetische druk kunnen zijn.

Ook keek ik naar de toekomst. Ik identificeerde enkele veelbelovende routes voor vervolgonderzoek, bij voorbeeld naar de invloed van de recente invoering van nationale kwaliteitsbewakingsstructuren op het beleid van universiteiten, of uitbreiding van de studie naar niet-universitaire hogeronderwijsinstellingen (TEI) in Griekenland. Een meer praktische uitkomst van mijn studie zou de ontwikkeling kunnen zijn van bureaus voor institutionele dataverzameling (institutional research).

In het huidige tijdsgewricht van economische crisis en partijpolitieke spanningen is het des te belangrijker om het hoger onderwijs in Griekenland te veranderen: voor efficiënt onderwijs zijn leiderschap en kwaliteitsmanagement meer dan ooit nodig. 


\section{Appendices}

\section{Appendix A Legal Basis}

\section{Article 16 of the Greek Constitution: Education, arts, science ${ }^{42}$}

1. Art and science, research and teaching shall be free and their development and promotion shall be an obligation of the State. Academic freedom and freedom of teaching shall not exempt anyone from his duty of allegiance to the Constitution.

2. Education constitutes a basic mission for the State and shall aim at the moral, intellectual, professional and physical training of Greeks, the development of national and religious consciousness and at their formation as free and responsible citizens.

3. The number of years of compulsory education shall be no less than nine.

4. All Greeks are entitled to free education on all levels at State educational institutions. The State shall provide financial assistance to those who distinguish themselves, as well as to students in need of assistance or special protection, in accordance with their abilities.

5. Education at university level shall be provided exclusively by institutions which are fully selfgoverned public law legal persons. These institutions shall operate under the supervision of the State and are entitled to financial assistance from it; they shall operate on the basis of statutorily enacted by-laws. Merging or splitting of university level institutions may take place notwithstanding any contrary provisions, as a law shall provide. A special law shall define all matters pertaining to student associations and the participation of students therein.

6. Professors of university level institutions shall be public functionaries. The remaining teaching personnel likewise perform a public function, under the conditions specified by law. The statutes of respective institutions shall define matters relating to the status of all the above. Professors of university level institutions shall not be dismissed prior to the lawful termination of their term of service, except in the cases of the substantive conditions provided by article 88 paragraph 4 and following a decision by a council constituted in its majority of highest judicial functionaries, as specified by law. The retirement age of professors of university level institutions shall be determined by law; until such law is issued, professors on active service shall retire ipso jure at the end of the academic year at which they have reached the age of sixty-seven.

7. Professional and any other form of special education shall be provided by the State, through schools of a higher level and for a time period not exceeding three years, as specifically provided by law which also defines the professional rights of the graduates of such schools.

8. The conditions and terms for granting a license for the establishment and operation of schools not owned by the State, the supervision of such and the professional status of teaching personnel

\footnotetext{
42 Hellenic Parliament 2004, THE CONSTITUTION OF GREECE As revised by the parliamentary resolution of April 6th 2001 of the VIIth Revisionary Parliament, Translated by Xenophon Paparrigopoulos \& Stavroula Vassilouni, p. 30. http://www.nis.gr/npimages/docs/Constitution_EN.pdf
} 
therein shall be specified by law. The establishment of university level institutions by private persons is prohibited.

9. Athletics shall be under the protection and the ultimate supervision of the State. The State shall make grants to and shall control all types of athletic associations, as specified by law. The use of grants in accordance with the purpose of the associations receiving them shall also be specified by law. 


\section{Appendix B Questionnaire and Tables on Malcolm Baldrige Study}

\section{B1- Greek Questionnaire original version

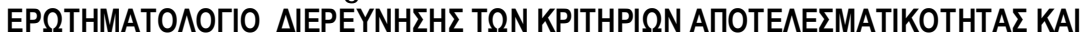

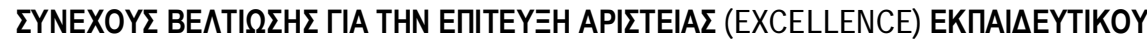 IDPYMATOE}

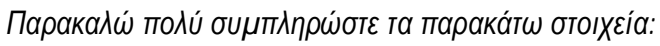

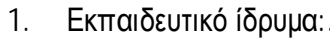

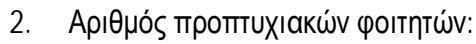

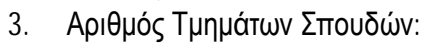

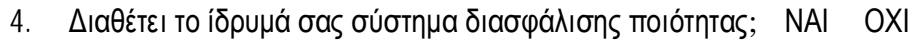

5. Фúৗo: Avঠopas Гuvaíka

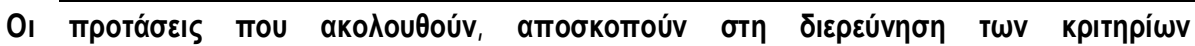

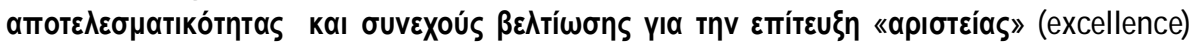

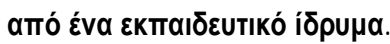

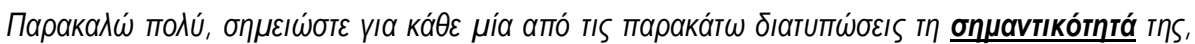

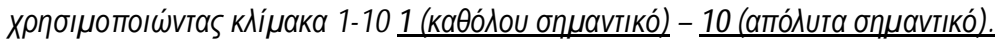

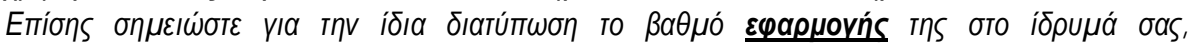

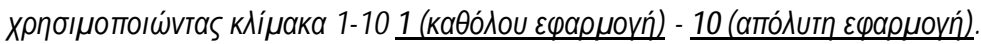

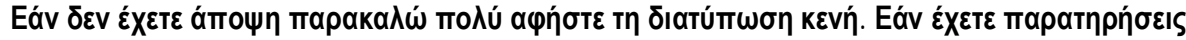

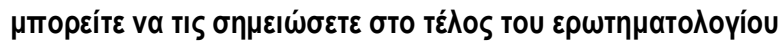

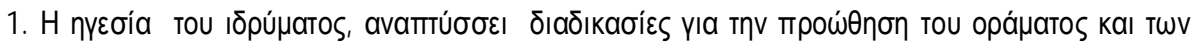

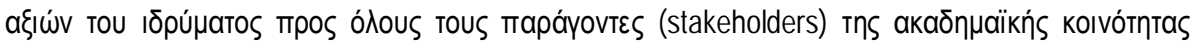
(

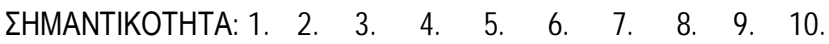

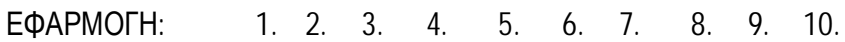

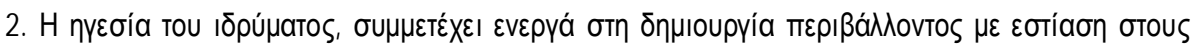

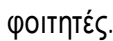

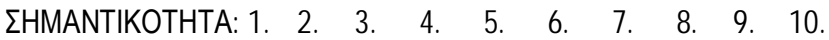

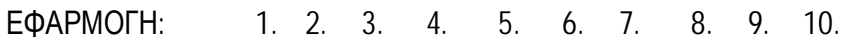

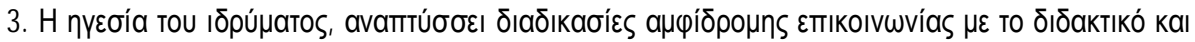

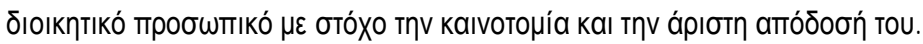

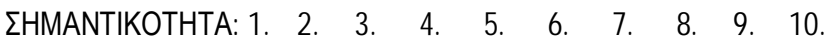

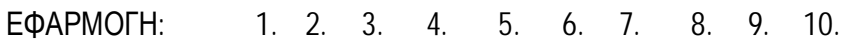

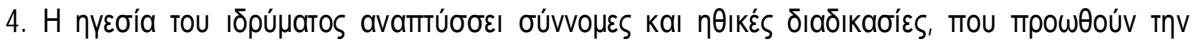

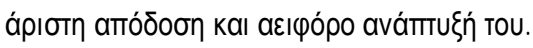

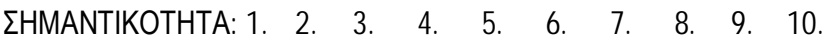




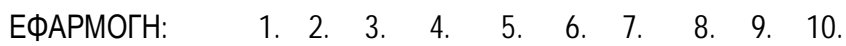

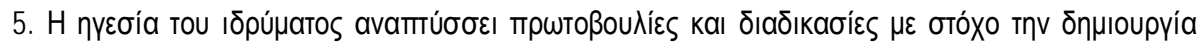

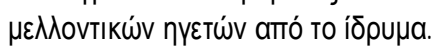

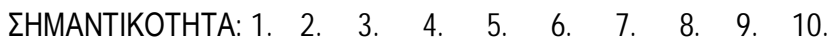

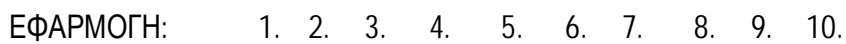

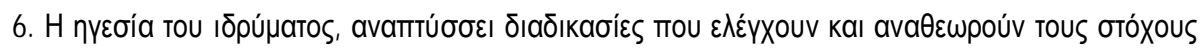

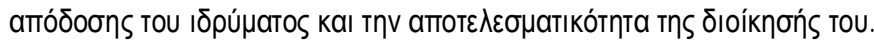

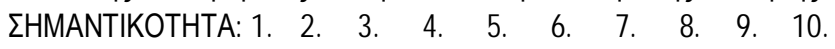

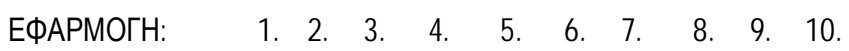

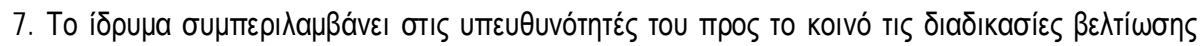

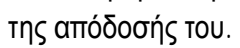

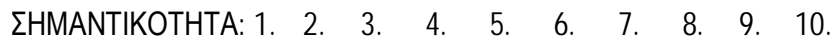

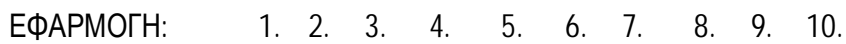

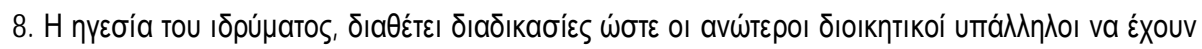

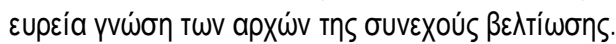

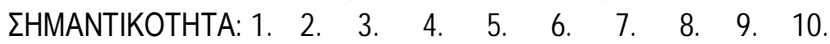

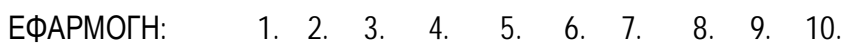

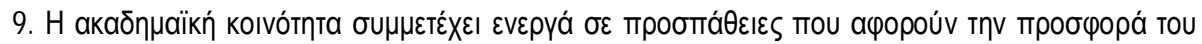

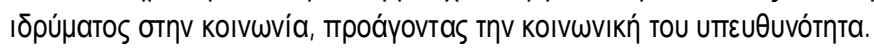

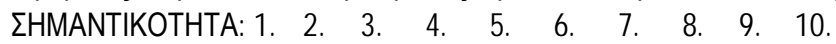

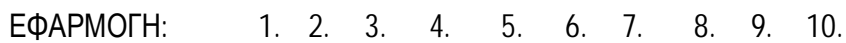

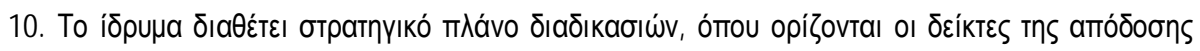

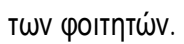

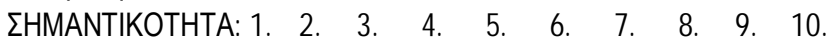

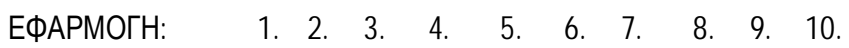

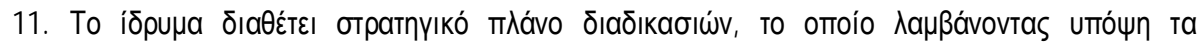

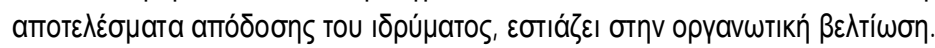

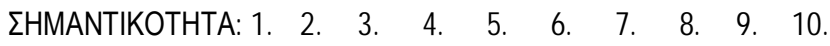

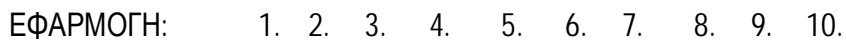

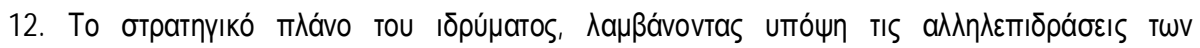

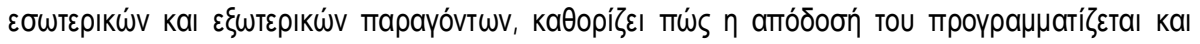

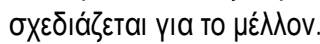

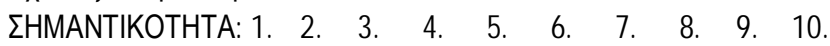

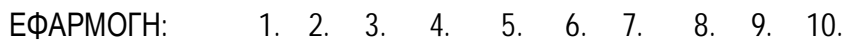




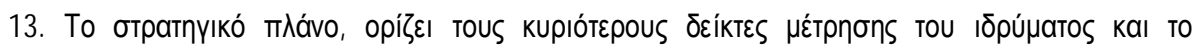

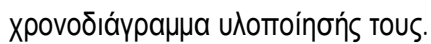

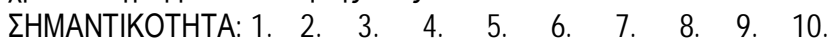

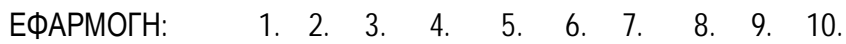

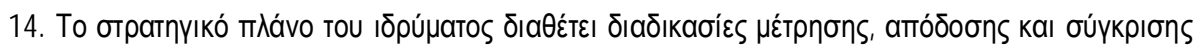

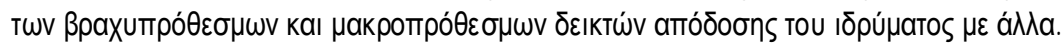

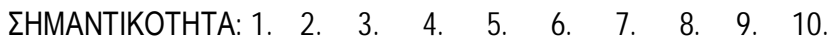

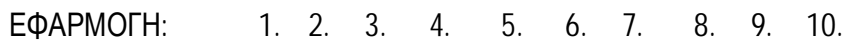

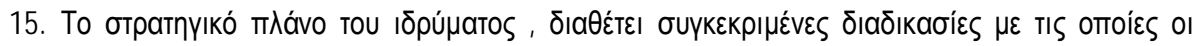

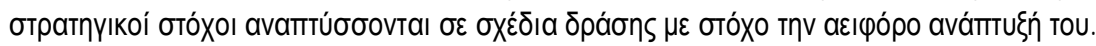

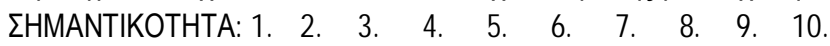

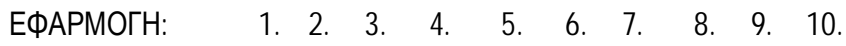

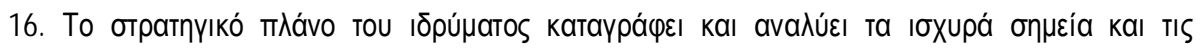

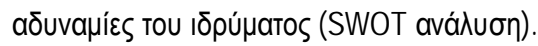

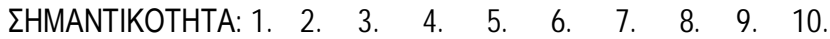

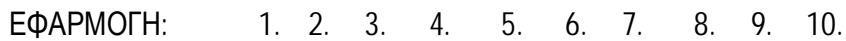

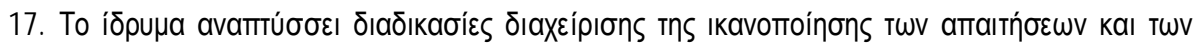

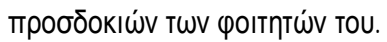

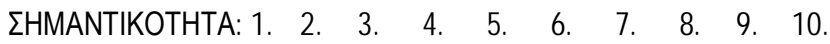

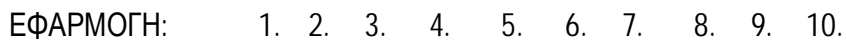

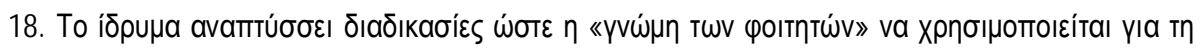

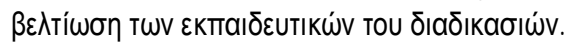

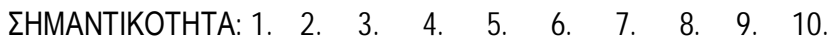

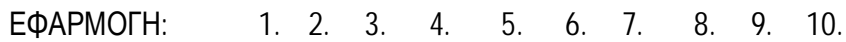

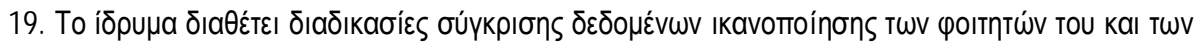

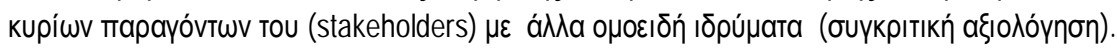

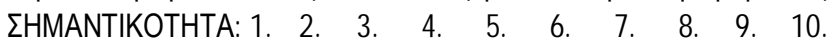

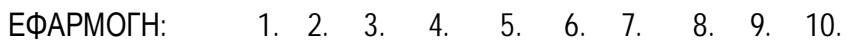

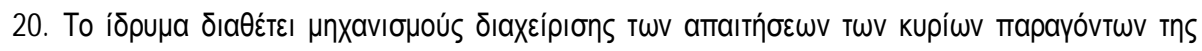

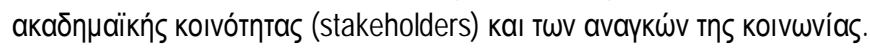

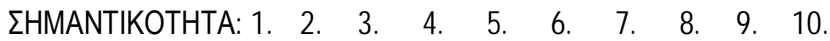

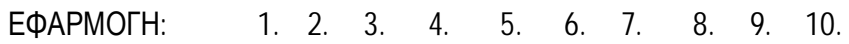




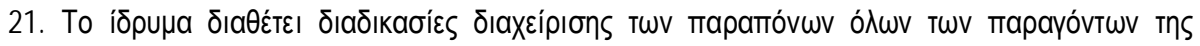

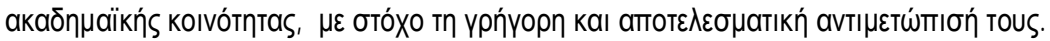

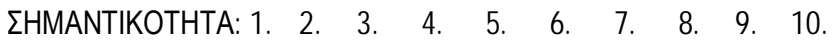

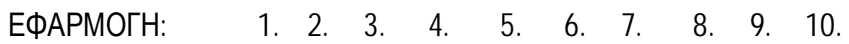

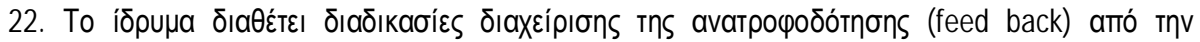

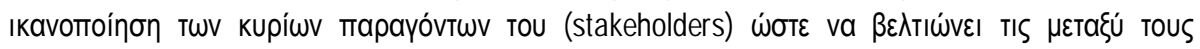

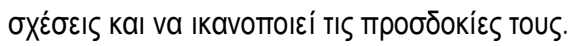

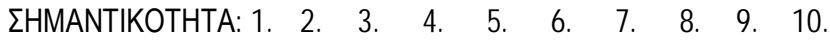

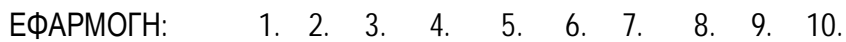

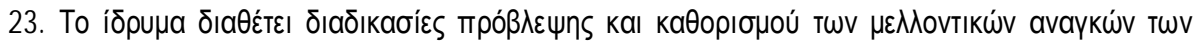
पOITпTÚV TOU.

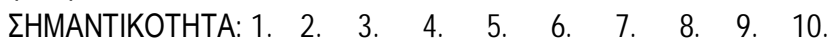

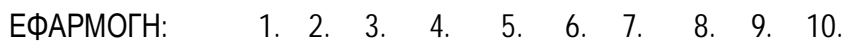

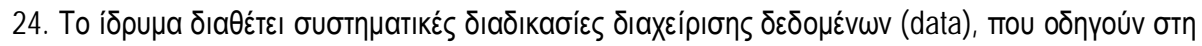

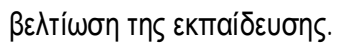

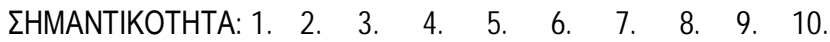

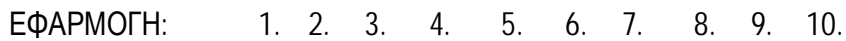

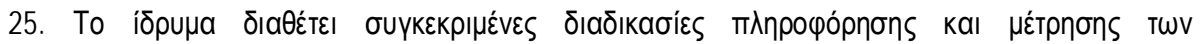

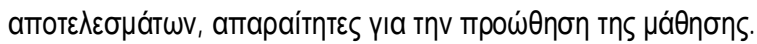

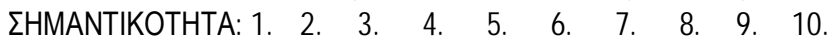

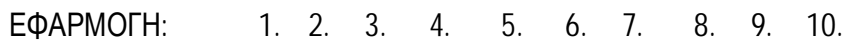

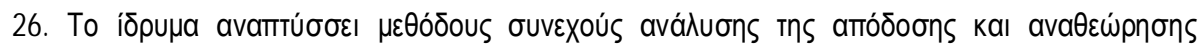

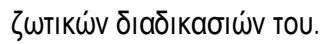

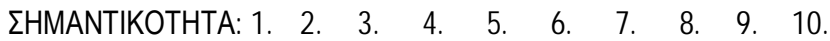

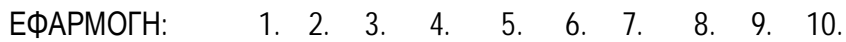

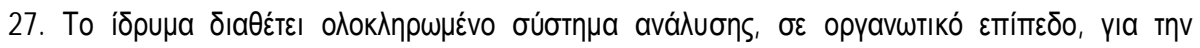

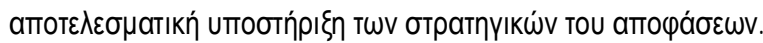

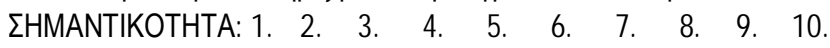

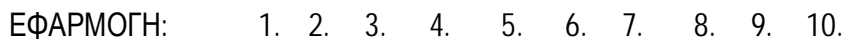

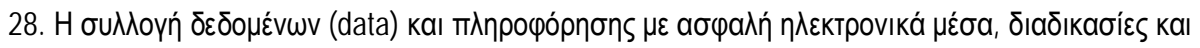

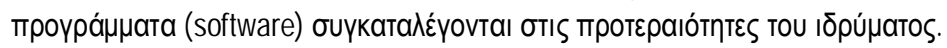

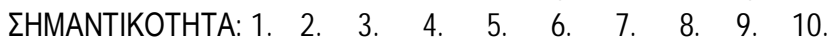

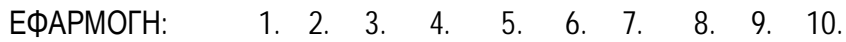




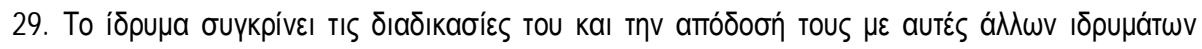

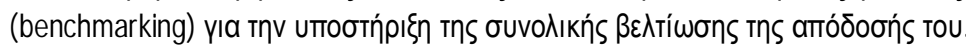

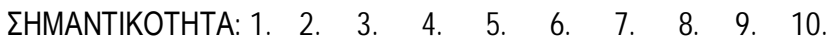

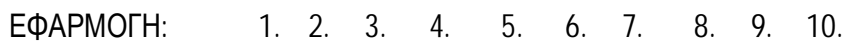

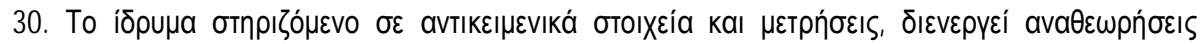

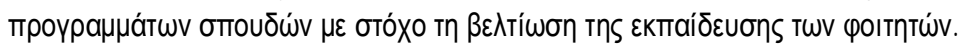

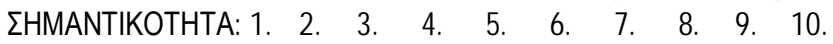

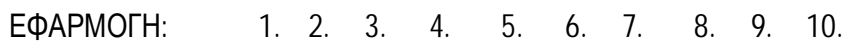

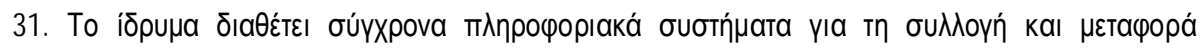

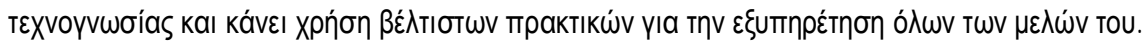

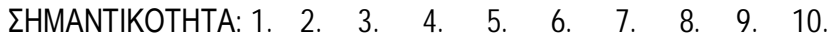

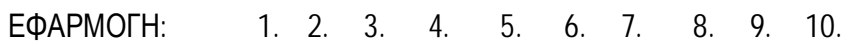

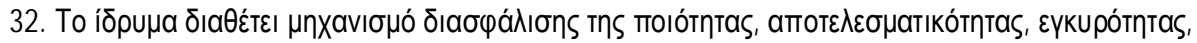

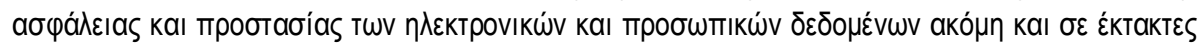

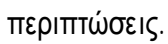

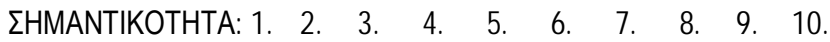

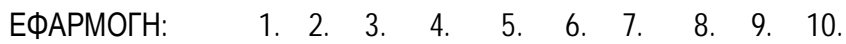

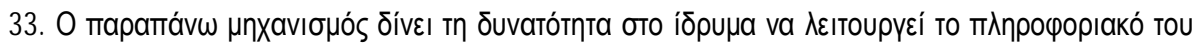

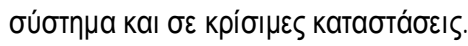

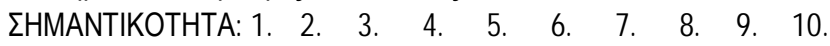

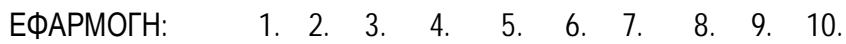

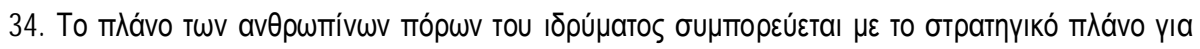

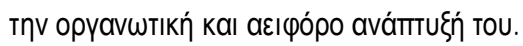

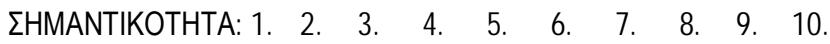

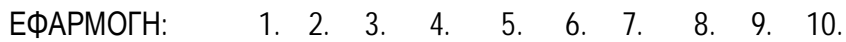

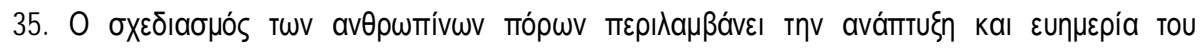

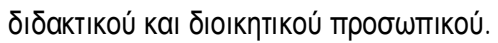

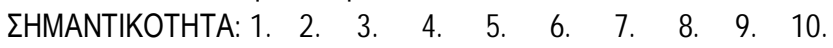

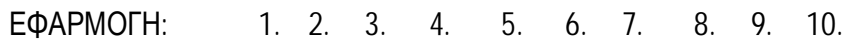

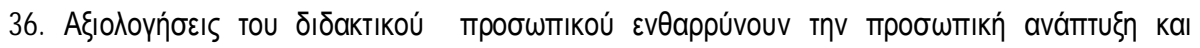
$\beta \varepsilon \lambda T i ́ \omega \sigma \eta$.

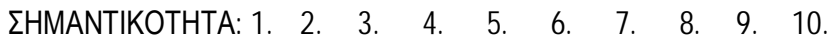

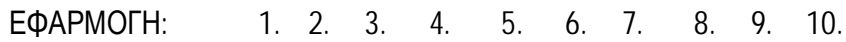




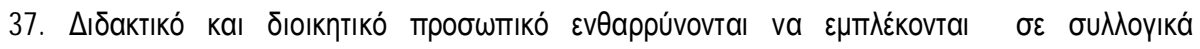

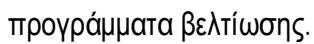

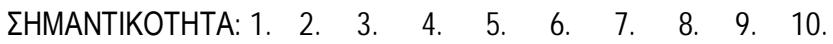

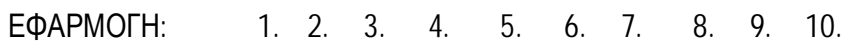

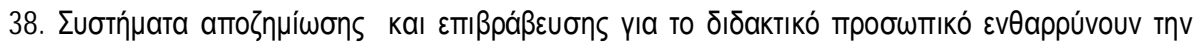

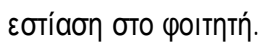

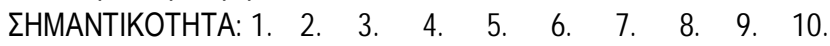

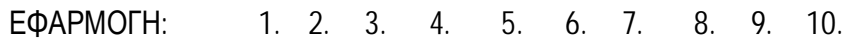

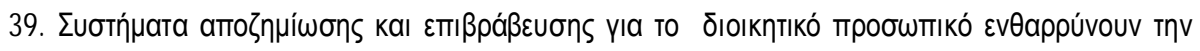

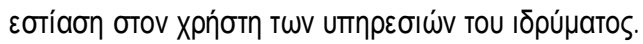

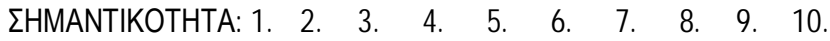

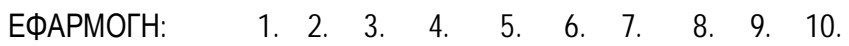

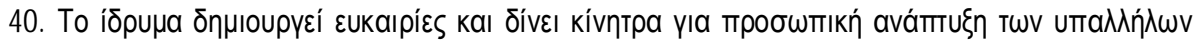
TOU.

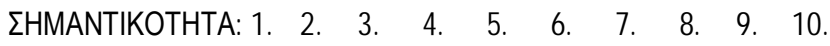

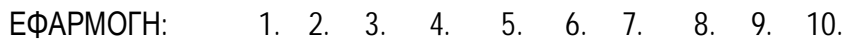

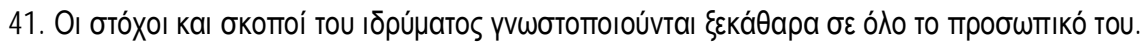

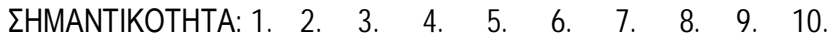

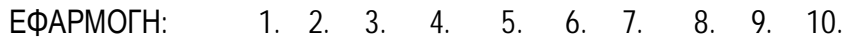

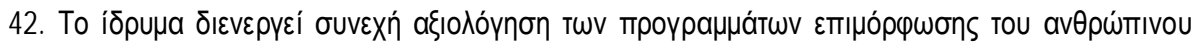

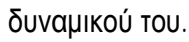

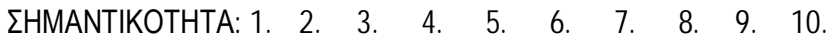

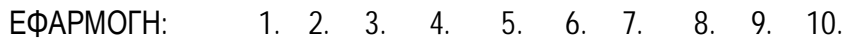

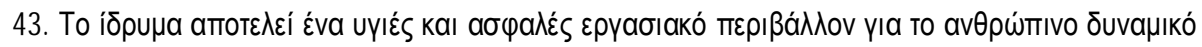
TOU.

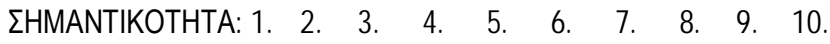

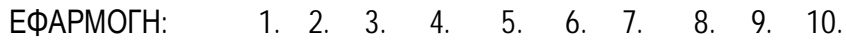

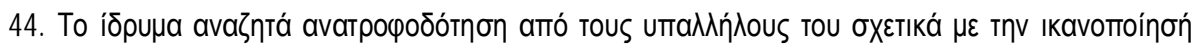

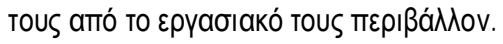

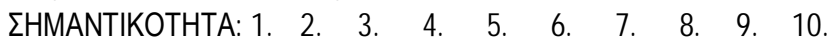

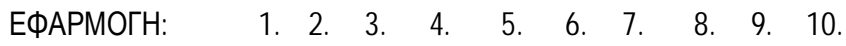

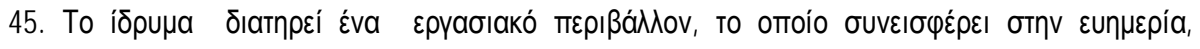

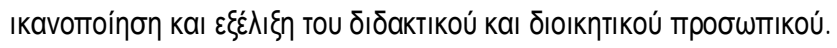

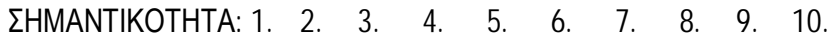




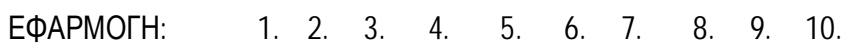

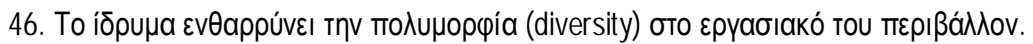

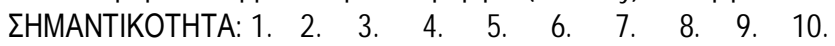

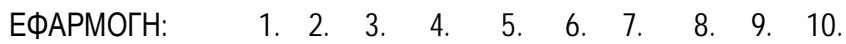

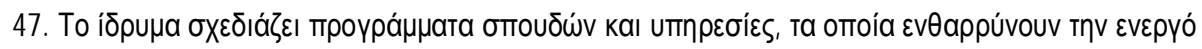

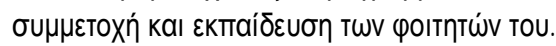

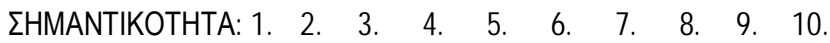

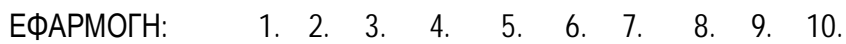

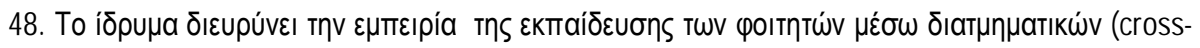

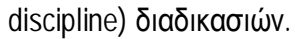

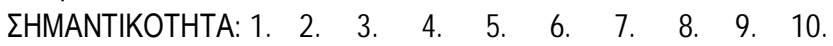

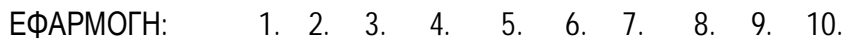

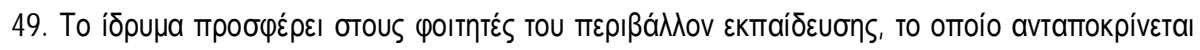

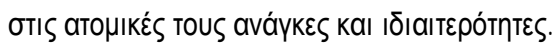

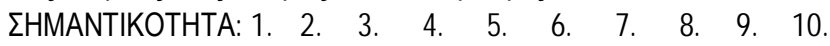

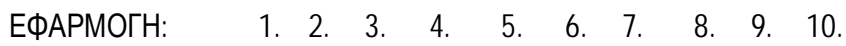

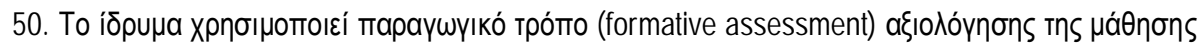

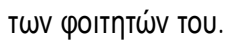

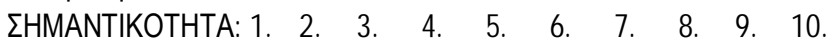

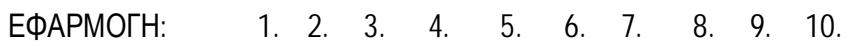

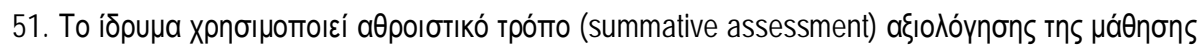

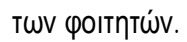

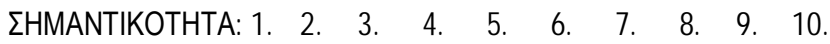

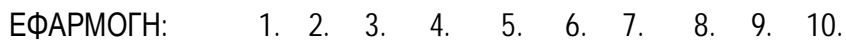

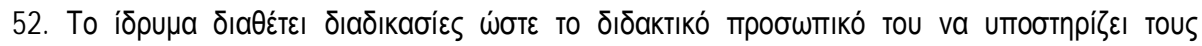

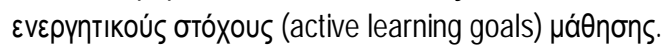

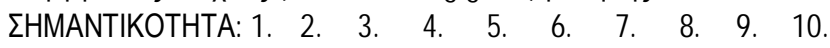

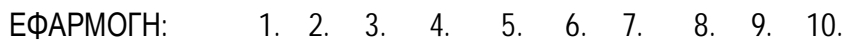

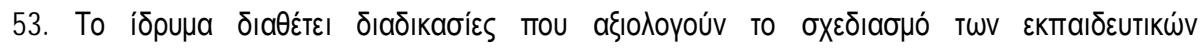

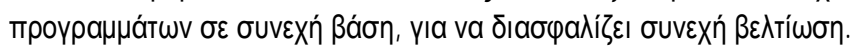

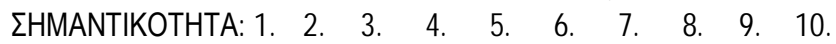

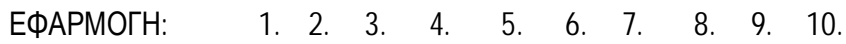




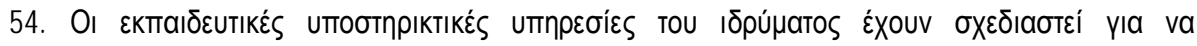

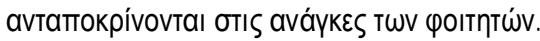

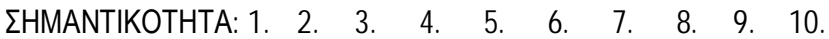

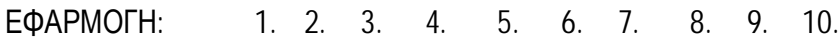

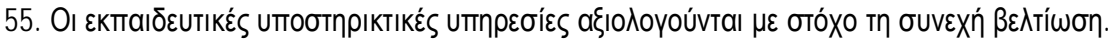

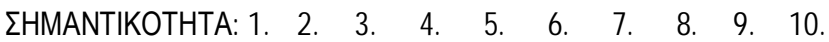

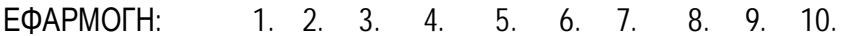

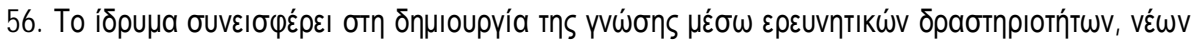

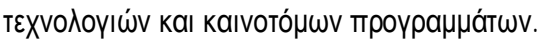

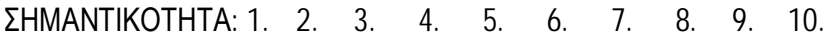

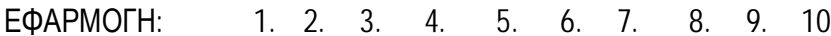

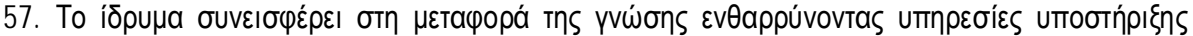

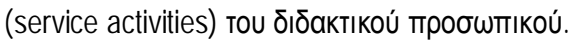

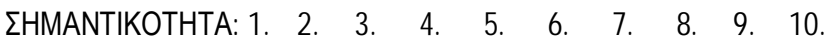

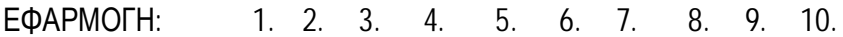

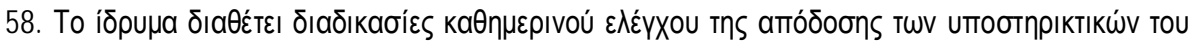

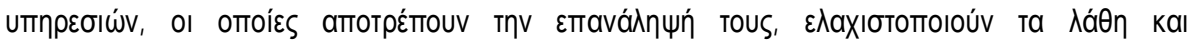

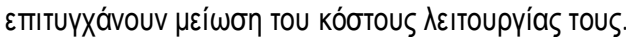

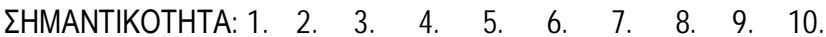

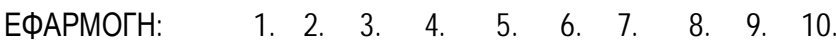

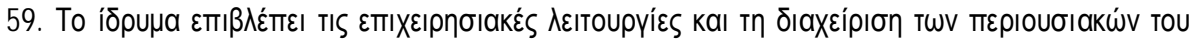

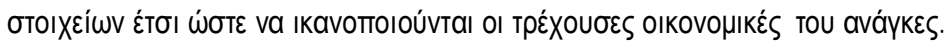

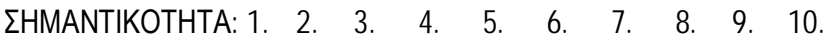

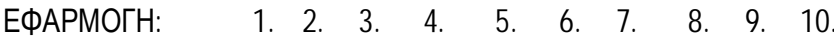

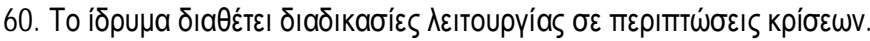

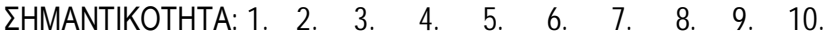

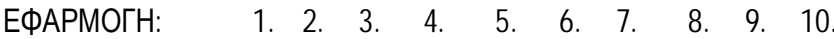

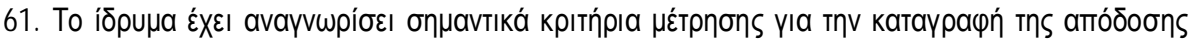

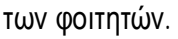

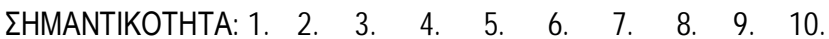

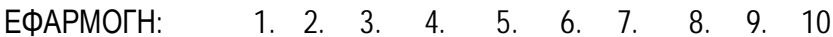

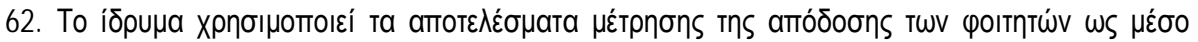

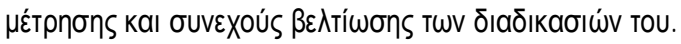

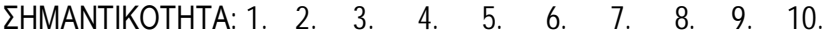




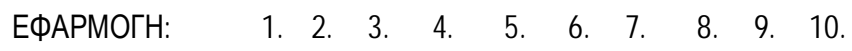

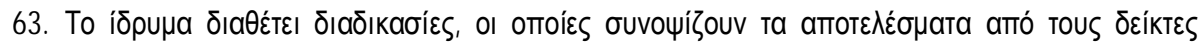

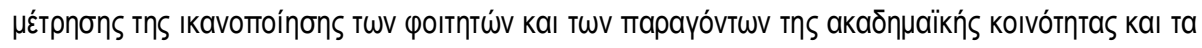

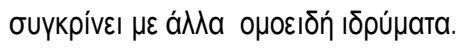

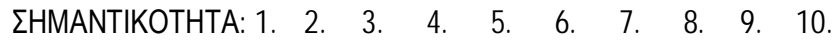

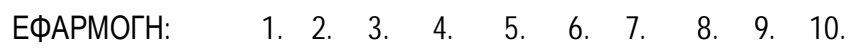

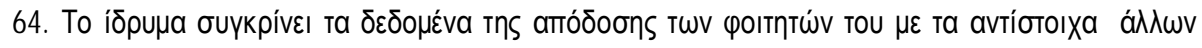

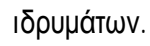

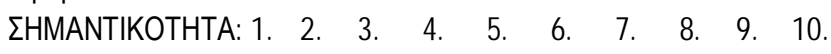

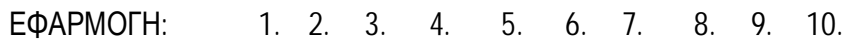

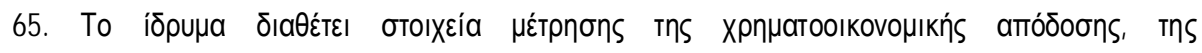

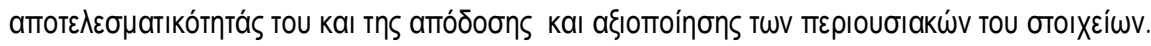

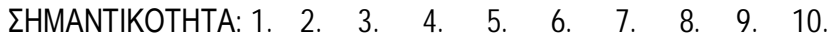

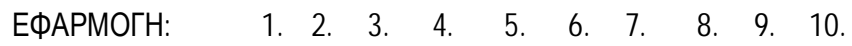

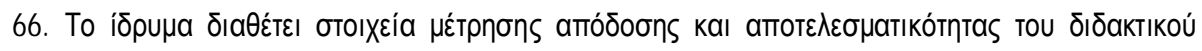
пробштाкои́.

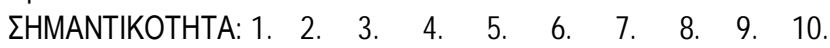

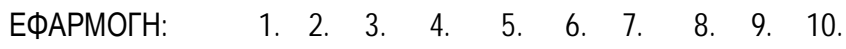

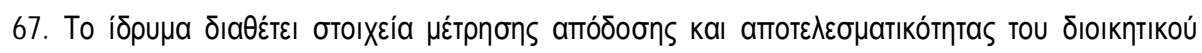
пробштाкои́.

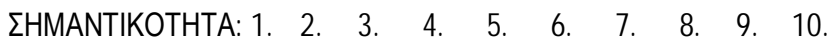

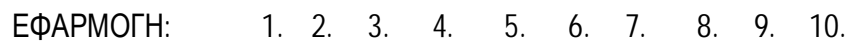

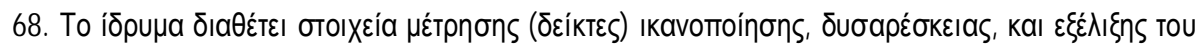

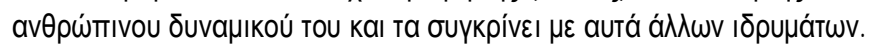

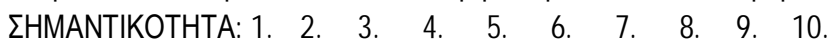

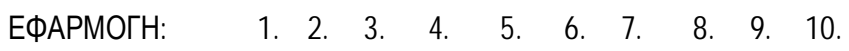

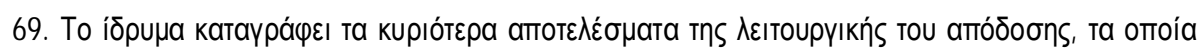

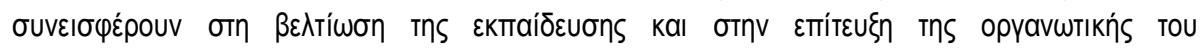

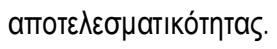

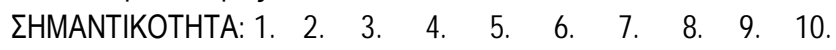

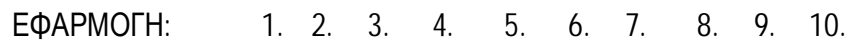

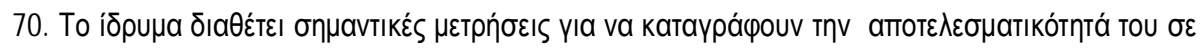
Өह́ната :

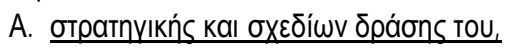




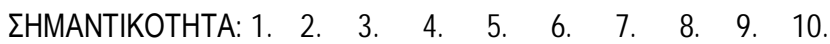
ЕФАРМОГН:
1. 2. 3. 4. 5.6 .6 . 7 .
8. 9.10.

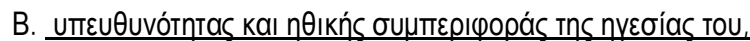

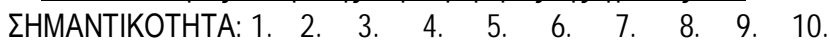

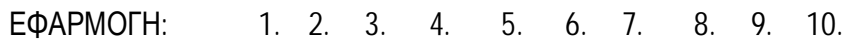

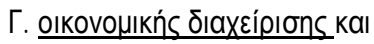

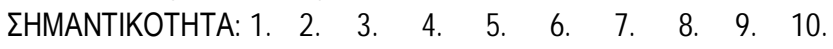

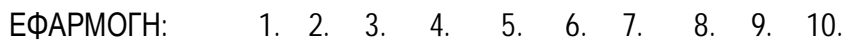

$\triangle$. KOIVWVIKńs UTEUӨUVótnTas.

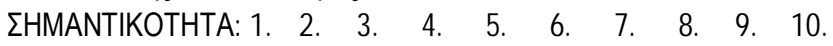

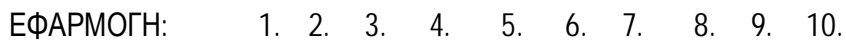

\section{B2- Translation: Questionnaire to Investigate the Criteria of Effectiveness and Continues Improvement for Achieving Excellence of an Educational Institution}

\section{Questions/statements}

1. The leadership of the institution develops procedures for the promotion of the vision and the principles of the institution towards all the stakeholders of the academic community (students, Academic Staff, Administrative Staff, graduates, society).

2. The leadership of the institution participates actively in the formation of an environment with focus on the students.

3. The leadership of the institution develops procedures of mutual communication with the academic and administrative staff aiming to innovate and perfect its performance.

4. The leadership of the institution develops legal and moral procedures, which promote its excellent performance and its continuous progress.

5. The leadership of the institution develops initiatives and procedures aiming to generate the future leaders from the institution.

6. The leadership of the institution develops procedures, which control and reconsider the performance goals of the institution and the efficacy of its management.

7. The institution includes within its responsibilities to the public the procedures of its performance improvement.

8. The leadership of the institution employs processes such that upper administrative staff has broad knowledge of the principles of continuous improvement.

9. The academic community participates actively in efforts, which regard the contribution of the institution in society, promoting its social responsibility.

10. The institution has a strategic plan of procedures, where indicators of students' performance are defined.

11. The institution has a strategic plan of procedures, which taking into account the performance results of the institution, focuses on organizational improvement.

12. The strategic plan of the institution, taking into account the interplay of domestic and foreign factors, determines how its performance is programmed and planned in the future. 
13. The strategic plan defines the major measurement indicators of the institution and the timetable of their implementation.

14. The strategic plan of the institution has procedures of measurement, performance, and comparison of the short-term and long-term performance indicators of the institution with other institutions.

15. The strategic plan of the institution has specific processes with which its strategic goals are developed along action plans aiming to its continuous development.

16. The strategic plan of the institution records and analyses the strengths and the weaknesses of the institution (SWOT analysis).

17. The institution develops management procedures for the satisfaction of the demands and the ambitions of its students.

18. The institution develops procedures such that "students' opinions" are used for the improvement of its academic procedures.

19. The institution has procedures to compare the satisfaction of its students and its major agents (stakeholders) with other respective institutions.

20. The institution has management mechanisms for the demands of the academic community stakeholders and the needs of the society.

21. The institution has management procedures for the complaints of all the academic community agents, aiming to their rapid and effective confrontation.

22. The institution has management procedures for the feedback from the satisfaction of its stakeholders in order to improve its relations with them and satisfy their ambitions.

23. The institution has procedures for the foreseeing and the determination of the future needs of its students.

24. The institution has systematic procedures of data management, which lead to the improvement of the education.

25. The institution has specific procedures of information and measurement of the results, indispensable for the promotion of learning.

26. The institution develops methods of continuous analysis of the performance and reconsideration of some of its vital procedures.

27. The institution has integrated system of analysis, at an organizational level, for the efficacious support of its strategic decisions.

28. The data and information collection with safe electronic means, procedures and programmes (software) are among the priorities of the institution.

29. The institution compares the procedures and their performance with those of other institutions (benchmarking) for the strengthening of the total improvement of its performance.

30. The institution sustained upon objective criteria and measures conducts amendments to its programme studies aiming to improve the education of its students.

31. The institution has modern information systems for the collection and transfer of knowhow and makes use of best practices for the service of all its members.

32. The institution has mechanism of assurance of quality, efficacy, validity and safety of electronic and personal data even in extraordinary cases. 
33. The above mechanism gives the opportunity to the institution to function its information system even in crucial occasions.

34. The plan of human resources of the institution goes along with the strategic plan for its organizational and continuous development.

35. The planning of human resources includes the progress and prosperity of its academic and administrative staff.

36. Evaluations of academic staff encourage personal development and improvement.

37. Academic and administrative staff is encouraged to get involved in collective improvement programmes.

38. Reimbursement and award systems for the academic staff encourage the focus on the student.

39. Reimbursement and award systems for the administrative staff encourage the focus on the student.

40. The institution creates opportunities and gives motives for personal progress of its employees.

41. The aims and the goals of the institution are known clearly to its staff.

42. The institution conducts continuous evaluation of its personnel training programmes.

43. The institution constitutes a vigorous and safe working environment for its personnel.

44. The institution looks for feedback for its employees with regard to their satisfaction from their working environment.

45. The institution maintains a working environment, which contributes to the prosperity, satisfaction and evolution of the academic and administrative staff.

46. The institution encourages the diversity in its working environment.

47. The institution plans programme studies and services, which encourage the active participation and education of its students.

48. The institution broadens the experience of students' education by cross-discipline procedures.

49. The institution offers to its students an educational environment, which corresponds to their personal needs and priorities.

50. The institution uses formative assessment for the evaluation of its students' learning.

51. The institution uses summative assessment for the evaluation of students' learning.

52. The institution has procedures so that its academic staff can support the active learning goals of learning.

53. The institution has procedures which evaluate the scheduling of educative programmes in a continuous basis in order to ensure constant improvement.

54. The educational supportive services of the institution have been scheduled in order to keep pace with students' needs.

55. The educational supportive services are evaluated aiming to continuous improvement.

56. The institution contributes to the creation of knowledge by research activities, new technologies and innovative projects.

57. The institution contributes to the transfer of knowledge encouraging reinforcing service activities for the academic staff. 
58. The institution has services of every-day control of the performance of its reinforcing services, which prevent their replication, minimise the errors and achieve the decrease of their function cost.

59. The institution supervises the business functions and the management of its property so that its current financial needs are satisfied.

60. The institution has work procedures in case of crisis.

61. The institution has recognized important criteria of measurement for the recording of the performance of the students.

62. The institution uses the measurement results of students as a means of measurement a constant improvement of its procedures.

63. The institution has procedures which summarise the results from students' and academics' satisfaction measurement indicators and compares them with other similar institutions.

64. The institution compares the students' performance data with the corresponding ones of other institutions.

65. The institution has data for the measurement of its financial performance, efficacy and performance and effective use of its property.

66. The institution has data for the measurement of the performance and competence of academic staff.

67. The institution has data for the measurement of the performance and the competence of its administrative staff.

68. The institution has measurement indicators for the satisfaction, discontent and progress of its human resource and compares them with those of other institutions.

69. The institution records the major results of its functional performance, which contribute to the improvement of the education and the achievement of its organizational efficiency.

70. The institution has important measures for the recording of its efficacy in issues of:
a. scheduling and activity plans
b. responsibility and moral behavior of its leadership
c. Financial management and
d. Social responsibility 
Table B1 Non-responses (importance scale) per MB category by university

\begin{tabular}{|c|c|c|c|c|c|c|c|c|c|c|c|c|}
\hline MB Categories & U1 & U2 & U3 & U4 & U5 & U6 & U7 & U8 & U9 & Total & $\begin{array}{l}\text { Non-response } \\
\text { as } \% \text { of all } \\
\text { questions }\end{array}$ & Item\# \\
\hline 1. Leadership & & & & 1 & & 1 & & & & 2 & $2 \%$ & $9=81$ \\
\hline 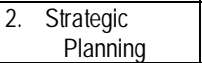 & & 1 & & 1 & & & & & & 2 & $3 \%$ & $7=63$ \\
\hline $\begin{array}{l}\text { 3. } \\
\text { Student, } \\
\text { Stakeholders \& } \\
\text { Market focus }\end{array}$ & & & & & 1 & & & & & 1 & $1 \%$ & $9=81$ \\
\hline $\begin{array}{ll}\text { 4. } & \text { Measurement } \\
\text { Analysis \& } \\
\text { Knowledge } \\
\text { Management } \\
\end{array}$ & & 5 & & 8 & 11 & & & & & 24 & $16 \%$ & $17=153$ \\
\hline $\begin{array}{l}\text { 5. Faculty \& Staff } \\
\text { focus }\end{array}$ & 1 & & & 1 & 4 & & & & & 6 & $4 \%$ & $15=135$ \\
\hline \begin{tabular}{ll|} 
6. Process \\
Management \\
\end{tabular} & 1 & 1 & & 3 & 3 & & & & & 8 & $13 \%$ & $7=63$ \\
\hline $\begin{array}{l}\text { 7. Organizational } \\
\text { \& Performance } \\
\text { Results } \\
\end{array}$ & & & & 2 & 3 & & & & & 5 & $8 \%$ & $9=64$ \\
\hline Total/University & 2 & 7 & 0 & 16 & 22 & 1 & 0 & 0 & 0 & 48 & $8 \%$ & $\begin{array}{c}73 \times 9=6 \\
57\end{array}$ \\
\hline
\end{tabular}

Table B2 Non-responses (implementation scale) per MB category by university

\begin{tabular}{|c|c|c|c|c|c|c|c|c|c|c|c|c|}
\hline MB Categories & U1 & U2 & U3 & U4 & U5 & U6 & U7 & U8 & U9 & Total & $\begin{array}{c}\text { Non- } \\
\text { response } \\
\text { as \% of all } \\
\text { questions } \\
\end{array}$ & Item\# \\
\hline 1. Leadership & & & & 1 & & 2 & & & & 3 & $4 \%$ & $9=81$ \\
\hline $\begin{array}{ll}\text { 2. } & \text { Strategic } \\
& \text { Planning }\end{array}$ & & 1 & & 1 & & & & & & 2 & $3 \%$ & $7=63$ \\
\hline $\begin{array}{l}\text { 3. Student, } \\
\text { Stakeholders } \\
\text { \& Market } \\
\text { focus }\end{array}$ & & & & & 1 & & & & & 1 & $1 \%$ & $9=81$ \\
\hline $\begin{array}{ll}\text { 4. } & \text { Measurement } \\
\text { Analysis \& } \\
\text { Knowledge } \\
\text { Management }\end{array}$ & & 6 & & 8 & 11 & 1 & & & & 26 & $17 \%$ & $\begin{array}{c}17=15 \\
3\end{array}$ \\
\hline $\begin{array}{l}\text { 5. Faculty \& Staff } \\
\text { focus }\end{array}$ & 1 & 1 & & 1 & 4 & 4 & & & & 11 & $8 \%$ & $\begin{array}{c}15=13 \\
5\end{array}$ \\
\hline $\begin{array}{ll}6 . & \begin{array}{l}\text { Process } \\
\text { Management }\end{array}\end{array}$ & 1 & 2 & & 3 & 3 & 1 & & & & 10 & $16 \%$ & $7=63$ \\
\hline $\begin{array}{ll}\text { 7. } & \text { Organizational } \\
\& \\
\text { Performance } \\
\text { Results }\end{array}$ & & & & 2 & 3 & & & & & 5 & $9 \%$ & $9=64$ \\
\hline Total/University & 2 & 10 & 0 & 16 & 22 & 8 & 0 & 0 & 0 & 58 & $9 \%$ & $\begin{array}{c}70 \times 9= \\
657\end{array}$ \\
\hline
\end{tabular}




\begin{tabular}{|c|c|c|c|c|c|c|c|c|c|}
\hline Universities: & 1 & 2 & 3 & 4 & 5 & 6 & 7 & 8 & 9 \\
\hline \multicolumn{10}{|c|}{ Category 1 Leadership } \\
\hline Importance & 9,2 & 8,3 & 9,3 & 8,1 & 7,1 & 9,0 & 8,9 & 10,0 & 9,7 \\
\hline Implementation & 7,6 & 2,2 & 7,6 & 7,8 & 5,7 & 5,9 & 6,0 & 2,7 & 6,8 \\
\hline Difference/Gap & $-1,7$ & $-6,1$ & $-1,8$ & $-0,3$ & $-1,4$ & $-3,1$ & $-2,9$ & $-7,3$ & $-2,9$ \\
\hline \multicolumn{10}{|c|}{ Category 2 Strategic planning } \\
\hline Importance & 8,6 & 7,9 & 9,0 & 7,3 & 6,0 & 10,0 & 8,7 & 10,0 & 9,0 \\
\hline Implementation & 6,1 & 2,4 & 7,0 & 5,3 & 3,1 & 1,3 & 6,4 & 2,6 & 6,1 \\
\hline Difference/Gap & $-2,4$ & $-5,4$ & $-2,0$ & $-2,0$ & 2,9 & $-8,7$ & $-2,3$ & $-7,4$ & $-2,9$ \\
\hline \multicolumn{10}{|c|}{ Category 3 Student Stakeholders and market Focus } \\
\hline Importance & 8,2 & 7,2 & 9,6 & 8,0 & 5,8 & 10,0 & 8,8 & 9,7 & 9,6 \\
\hline Implementation & 6,2 & 2,0 & 8,0 & 7,4 & 4,7 & 5,2 & 7,0 & 4,8 & 5,4 \\
\hline Difference/Gap & $-2,0$ & $-5,2$ & $-1,6$ & $-0,6$ & 1,1 & $-4,8$ & $-1,8$ & $-4,9$ & $-4,1$ \\
\hline \multicolumn{10}{|c|}{ Category 4 Measurement Analysis and Knowledge Management } \\
\hline Importance & 8,9 & 5,7 & 9,7 & 4,5 & 3,3 & 10,0 & 8,7 & 10,0 & 9,8 \\
\hline Implementation & 5,9 & 1,7 & 8,0 & 4,0 & 2,6 & 3,9 & 7,4 & 3,9 & 6,9 \\
\hline Difference/Gap & $-3,0$ & $-4,0$ & $-1,7$ & $-0,5$ & 0,7 & $-6,1$ & $-1,3$ & $-6,1$ & $-2,9$ \\
\hline \multicolumn{10}{|c|}{ Category 5 Faculty and Staff Focus } \\
\hline Importance & 8,9 & 8,1 & 9,5 & 7,5 & 5,8 & 10,0 & 8,9 & 10,0 & 10,0 \\
\hline Implementation & 6,7 & 2,3 & 7,6 & 6,8 & 5,2 & 5,7 & 7,1 & 4,2 & 7,5 \\
\hline Difference/Gap & $-2,3$ & $-5,8$ & $-1,9$ & $-0,7$ & 0,6 & $-4,3$ & $-1,7$ & $-5,8$ & $-2,5$ \\
\hline \multicolumn{10}{|c|}{ Category 6 Process Management } \\
\hline Importance & 7,9 & 7,3 & 9,9 & 4,7 & 5,3 & 10,0 & 9,0 & 10,0 & 10,0 \\
\hline Implementation & 6,3 & 2,4 & 7,7 & 4,7 & 5,3 & 5,1 & 7,7 & 8,3 & 7,3 \\
\hline Difference/Gap & $-1,6$ & $-4,9$ & $-2,2$ & $-0,0$ & 0,0 & $-4,9$ & $-1,3$ & $-1,7$ & $-2,7$ \\
\hline \multicolumn{10}{|c|}{ Category 7 Organizational and Performance Results } \\
\hline Importance & 9,3 & 7,9 & 10,0 & 7,1 & 6,1 & 10,0 & 8,6 & 10,0 & 10,0 \\
\hline Implementation & 6,7 & 1,3 & 7,8 & 7,1 & 6,1 & 2,8 & 7,3 & 5,9 & 7,1 \\
\hline Difference/Gap & $-2,7$ & $-6,6$ & $-2,2$ & $-0,0$ & 0,0 & $-7,2$ & $-1,2$ & $-4,1$ & $-2,9$ \\
\hline
\end{tabular}

Table B3 Overall gap analysis 


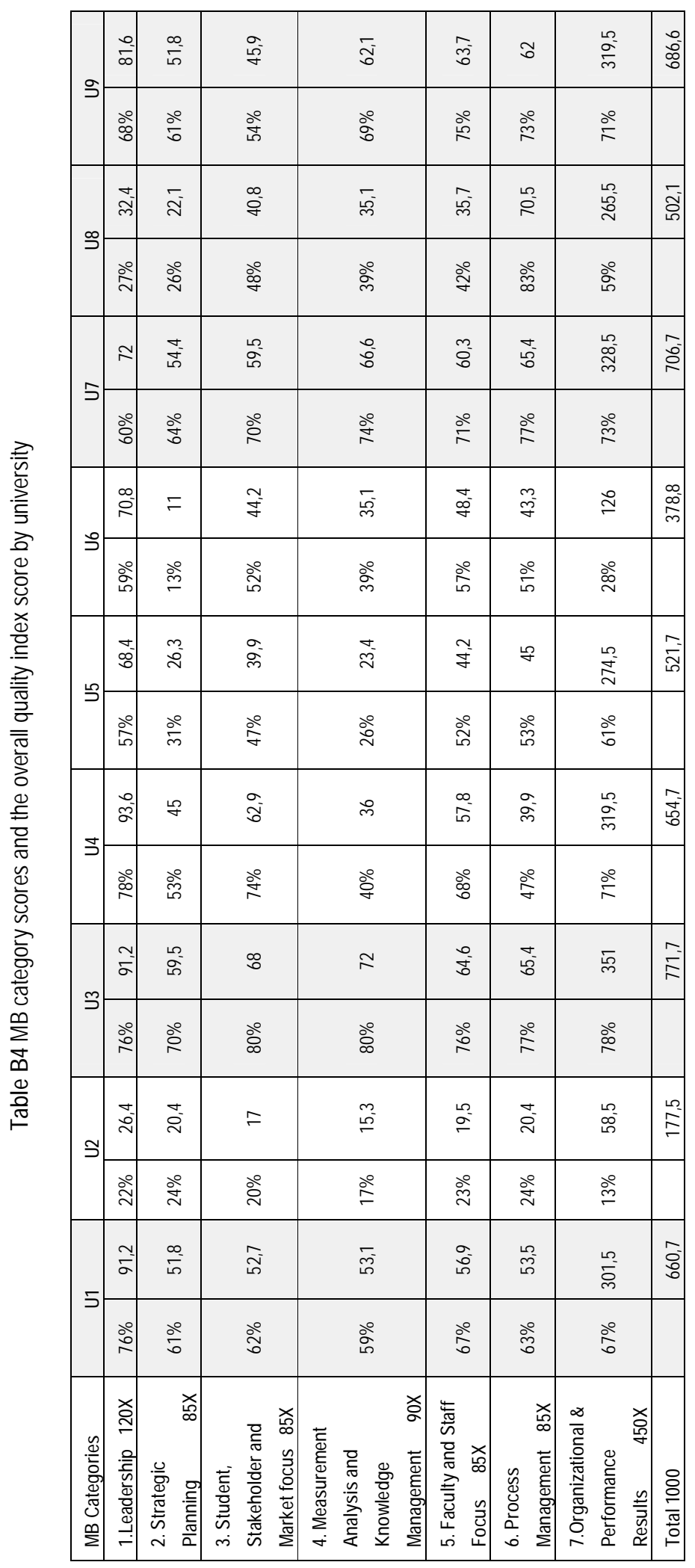




\title{
B3 Measuring Neo-Institutional Pressures in the Malcolm Baldrige Questionnaire
}

\author{
Normative pressure \\ i) Benchmarking
}

The survey items 19, 29, 63 and 64 are related with benchmarking ${ }^{43}$ approaches and suggest normative pressure.

ii) QM practices communications

In regards to normative pressure, as derived from the mentioned literature, if one communicates QM practices to all members of the university and it measured performance of the university according to QM objectives, this indicates a professional approach, and therefore has been interpreted as another sub-dimension of normative pressure. These issues are addressed with the questions 3, 6, 8, 10, 34, 37, 41, 42, 44, and 68 .

iii) Category 3 , Student Stakeholder and Market Focus.

Category 3 in MB, as mentioned earlier, examined how universities develop strategic objectives and action plans. It also examined how a university has chosen strategic objectives, how action plans are deployed, and how progress is measured. In other words, this category is perceived as normative pressure associated with professionalization. If any university adopts QM practices with the aim to focus on its students, parents, and market needs perhaps it suggests normative pressure. I should note that in Greece where education (undergraduate) is free - without tuition this pressure suggested normative pressure; in countries where students and parents pay tuition this pressure is associated with coercive pressure. Since Greek public universities remained state financed while conducting during this research, the total items from category 3 were perceived as normative pressure.

iv) $\quad$ Category 5, Faculty and Staff focus

Category 5, in MB as mentioned earlier, examined how a university's work systems as well as faculty and staff learning and motivation enabled faculty and staff to develop and utilize their full potential in alignment with the university's overall objectives and action plans. Also examined were the university's efforts to build and maintain a work environment with a faculty and staff support climate conducive to performance of excellence and to personal and organizational growth. This category, similar to category 3 , is associated with professionalization. If any university adopts QM practices focussed on its faculty and staff needs, perhaps this effort suggested normative pressure as well. It is important to note that during this approach, the normative pressure diagnosis was constructed from one combination measurement (mean score) by using survey items only once.

\footnotetext{
43 Benchmarking. Benchmarking is the process of identifying, understanding, and adapting outstanding practices from organizations anywhere in the world to help organizations to improve their performances. It is a highly respected practice in the business world. It is an activity that looks outward to find best practice and high performance and then measures actual business operations against those goals (Belle, 2000). Benchmarking can also include contacting "best in class" organizations and learn from them about (internal) process. There are several "clubs" in HE that are doing that, e.g. ESMU, CEMS (Alstete, 1996)
} 


\section{Mimetic pressure}

Survey items such as 14 and 31 might address mimetic pressure. Thus, when a university compares its performance indicators and measurement with other similar institutions, it suggests mimetic pressure. Moreover, this practice (comparing information indicators and measurement) would be proper if it were included in the university's strategic planning. However, this "statement" perhaps from one reader's "eye" could be interpreted as normative pressure. There are several reasons to interpret these questions as mostly mimetic pressure, primarily because, unlike most other questions in the MB survey, it stresses following "other universities", i.e. mimetic behavior. Secondly, if the response in the implementation rate had been high (which would have meant that the university pays attention to details ${ }^{44}$ of the implementation of performance measurement) it might perhaps suggest practices arising from normative pressure. In my case, where the universities' implementation rates were low, this item suggested that just a few respondents felt the need to copy other universities' performance, which should be interpreted as mimetic pressure. In even stronger terms than question 14 , if one makes use of other best practices (31), it would suggest mimetic pressure.

${ }^{44}$ Strang and Meyer, 1993, "Institutional conditions for diffusion". Theory and Society (22):487-511 


\section{Appendix C Questionnaire on Quality Assurance Systems in Basic Units}

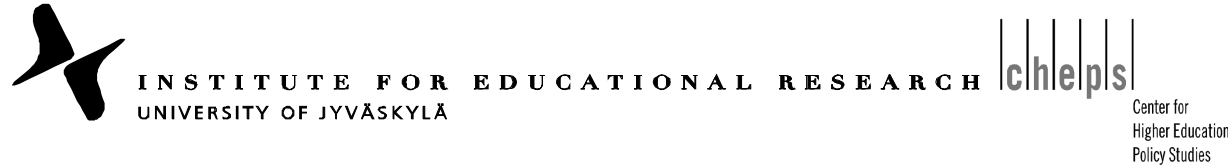

This questionnaire contains statements about a quality assurance system as an aspect of the functioning of your own Department. Please, circle or highlight (e.g. in a colour or bold) the number representing the alternative that best corresponds with your view on quality assurance systems or write your answer in the space provided. For each statement, you may select only one answer. Please, skip statements that you consider inapplicable to the operation of your Department/Unit or irrelevant in terms of your own view on quality assurance systems.

\begin{tabular}{|c|c|c|c|c|c|}
\hline & $\begin{array}{c}\text { Fully } \\
\text { disagree }\end{array}$ & Disagree & $\begin{array}{c}\text { Neither } \\
\text { agree nor } \\
\text { disagree }\end{array}$ & Agree & $\begin{array}{l}\text { Fully } \\
\text { agree }\end{array}$ \\
\hline $\begin{array}{l}\text { 1. I have no previous experience } \\
\text { of using quality assurance } \\
\text { systems }\end{array}$ & 1 & 2 & 3 & 4 & 5 \\
\hline $\begin{array}{l}\text { 2. Quality assurance systems are } \\
\text { highly suitable for teaching } \\
\text { development }\end{array}$ & 1 & 2 & 3 & 4 & 5 \\
\hline $\begin{array}{l}\text { 3. It is important to evaluate the } \\
\text { operational quality of our unit }\end{array}$ & 1 & 2 & 3 & 4 & 5 \\
\hline $\begin{array}{l}\text { 4. Quality assurance systems are } \\
\text { suitable for staff development } \\
\text { in our unit }\end{array}$ & 1 & 2 & 3 & 4 & 5 \\
\hline $\begin{array}{l}\text { 5. Quality assurance systems are } \\
\text { nothing but a waste of time } \\
\text { and extra bureaucracy }\end{array}$ & 1 & 2 & 3 & 4 & 5 \\
\hline $\begin{array}{l}\text { 6. The introduction of quality } \\
\text { assurance systems will } \\
\text { improve the operation of our } \\
\text { unit }\end{array}$ & 1 & 2 & 3 & 4 & 5 \\
\hline $\begin{array}{l}\text { 7. I know what is meant by quality } \\
\text { assurance systems }\end{array}$ & 1 & 2 & 3 & 4 & 5 \\
\hline $\begin{array}{l}\text { 8. Quality assurance systems are } \\
\text { highly suitable for developing } \\
\text { our unit's administrative } \\
\text { functions }\end{array}$ & 1 & 2 & 3 & 4 & 5 \\
\hline
\end{tabular}




\begin{tabular}{|c|c|c|c|c|c|}
\hline & $\begin{array}{c}\text { Fully } \\
\text { disagree }\end{array}$ & Disagree & $\begin{array}{c}\text { Neither } \\
\text { agree nor } \\
\text { disagree }\end{array}$ & Agree & $\begin{array}{l}\text { Fully } \\
\text { agree }\end{array}$ \\
\hline $\begin{array}{l}\text { 9. The introduction of a quality } \\
\text { assurance system will make it } \\
\text { easier to identify defects in the } \\
\text { operation of our unit }\end{array}$ & 1 & 2 & 3 & 4 & 5 \\
\hline $\begin{array}{l}\text { 10. Quality assurance systems are } \\
\text { not useful for developing the } \\
\text { operation of our unit. }\end{array}$ & 1 & 2 & 3 & 4 & 5 \\
\hline $\begin{array}{l}\text { 11. Quality assurance systems } \\
\text { make work tasks more } \\
\text { transparent }\end{array}$ & 1 & 2 & 3 & 4 & 5 \\
\hline $\begin{array}{l}\text { 12. I have never even heard of } \\
\text { university quality assurance } \\
\text { systems }\end{array}$ & 1 & 2 & 3 & 4 & 5 \\
\hline $\begin{array}{l}\text { 13. Our unit's existing evaluation } \\
\text { activities are a natural aspect } \\
\text { of the quality assurance } \\
\text { system }\end{array}$ & 1 & 2 & 3 & 4 & 5 \\
\hline $\begin{array}{l}\text { 14. I am acquainted with quality } \\
\text { assurance systems }\end{array}$ & 1 & 2 & 3 & 4 & 5 \\
\hline $\begin{array}{l}\text { 15. Quality assurance systems are } \\
\text { unsuitable for developing } \\
\text { academic work }\end{array}$ & 1 & 2 & 3 & 4 & 5 \\
\hline $\begin{array}{l}\text { 16. A quality assurance system } \\
\text { improves the legal protection } \\
\text { of the staff at our unit }\end{array}$ & 1 & 2 & 3 & 4 & 5 \\
\hline $\begin{array}{l}\text { 17. I know that universities must } \\
\text { evolve a quality assurance } \\
\text { system }\end{array}$ & 1 & 2 & 3 & 4 & 5 \\
\hline $\begin{array}{l}\text { 18. Quality assurance systems are } \\
\text { suitable for developing } \\
\text { educational guidance and } \\
\text { counselling }\end{array}$ & 1 & 2 & 3 & 4 & 5 \\
\hline $\begin{array}{l}\text { 19. I believe that staff at our unit } \\
\text { knows what is meant by quality } \\
\text { assurance systems }\end{array}$ & 1 & 2 & 3 & 4 & 5 \\
\hline $\begin{array}{l}\text { 20. Quality assurance systems are } \\
\text { completely unsuitable for } \\
\text { developing the operation of our } \\
\text { unit }\end{array}$ & 1 & 2 & 3 & 4 & 5 \\
\hline
\end{tabular}


21. Which of the developmental stages listed below best describes, in your opinion, the present state of the quality assurance system at your own unit (please select only one option)?

\begin{tabular}{|cl|ll|}
\hline 1 & Lacking. & 2 & Embryonic. \\
\hline 3 & Developing. & 4 & Advanced. \\
\hline 5 & Do not know. & & \\
\hline
\end{tabular}

22. Which of the developmental stages listed below best describes, in your opinion, the present state of the quality assurance system at your own university (please select only one option)?

\begin{tabular}{|ll|ll|}
\hline 1 & Lacking. & 2 & Embryonic. \\
\hline 3 & Developing. & 4 & Advanced. \\
\hline 5 & Do not know. & & \\
\hline
\end{tabular}

23. How would you define a quality assurance system from the perspective of your own unit?

24. In your opinion, do quality assurance systems benefit the operation of your unit?

1 No.

2 Do not know.

3 Yes, how? Write down the most important advantage.

25. In your opinion, do quality assurance systems hamper the operation of your unit?

1 No.

2 Do not know.

3 Yes, how? Write down the most important disadvantage.

26. As a quality assurance system is required by the law (3374/2005), do you think that a quality assurance system will be implemented in your department in the next:
1 year
2 years
3 years
never

27. As a quality assurance system will be part of the governmental funding process, do you think that a quality assurance system will be implemented in your department in the next:
1 year
2 years
3 years
never

28. Do you think that requests or requirements from employers of your graduates will lead to implementing a quality assurance system in your department in the next:

$$
1 \text { year } 2 \text { years } 3 \text { years never }
$$

29. Do you think that requests or requirements from students and parents will lead to implementing a quality assurance system in your department in the next:
1 year
2 years
3 years
never

30. Do you think that requests or requirements from your departmental members (faculty) will lead to implementing a quality assurance system in your department in the next:
1 year
2 years
3 years
never

31. If in your area $75 \%$ of the departments implemented a quality assurance system, do you think that you would implement a quality assurance system in your department in the next:
1 year
2 years
3 years
never

\section{BACKGROUND DETAILS}

32. My department belongs in the following discipline :

$\begin{array}{lcr}\text { Sciences } & \text { Engineering } & \text { Medicine and Life Sciences } \\ \text { Law } & \text { Behavioral Science } & \text { Social Science \& Economics } \\ \text { Humanities } & \text { Arts } & \end{array}$


33. My department is a part of:

\begin{tabular}{|c|r|ll|}
\hline 1 & National \& Kapodistrian University & 10 & University of Patras \\
& of Athens. & 11 & University of loannina Democritus \\
2 & National Technical University of & 12 & University of Thrace \\
& Athens. & 13 & University of Crete \\
3 & Aristotle University of & 14 & Technical University of Crete \\
& Thessaloniki. & 15 & University of the Aegean \\
4 & Athens University of Economics\& & 16 & Ionian University \\
& Business & 18 & University of Thessaly \\
5 & Agricultural University of Athens & 19 & University of Western Macedonia \\
6 & Pantion University & 20 & University of Peloponnese \\
7 & Athens School of Fine Arts & 21 & University of Continent Greece \\
8 & University of Piraeus & & \\
9 & University of Macedonia &
\end{tabular}

34. At the moment, there are people working at our department.

35. The questionnaire was filled in by

1 the department head.

2 someone else, namely

36. I am

1 a woman.

2 a man.

37. I have been employed at university for years (experience of working at university).

38. My present job title is

\begin{tabular}{|ll|ll|}
\hline 1 & Professor & 2 & Associate professor \\
\hline 3 & Assistant professor & 4 & Lecturer \\
\hline 5 & Department Secretary & 6 & Other title, please specify \\
\hline
\end{tabular}

39. I have taken part in training related to quality assurance systems in higher education.

1 Yes.

2 No.

Finally, if you wish, we offer you the opportunity to express your opinion on quality assurance systems, this questionnaire or this study. 


\title{
Appendix D Interview Protocol of Study into ISO Practices
}

\author{
Interview\# Date Time \\ Length of interview: \\ Interviewee Director: \\ Female/Male \\ Laboratory/service/unit: \\ Type of ISO:
}

\begin{tabular}{|l|l|l|l|}
\hline ISO 9001:2000 & ISO 17025 & EMAS & other \\
\hline
\end{tabular}

ISO process:

\begin{tabular}{|l|l|l|l|}
\hline First time & Second time & In process & No longer using ISO \\
\hline
\end{tabular}

1. The cost of this implementation is high. Do you believe that this is a disadvantage? Or there is no problem at all?

2. QM systems need human resources agreement and support; how do you manage this? Is this a problem?

3. Why did you apply for this quality management system? What was the motivation in order to adapt this practice?

Coercive

Mimetic

Normative (depending on the answer I follow-up with further questions related to my literature review to give more information regarding the type of pressure)

4. As director, did you have any involvement from the beginning of this application? Who decided on these standards

5. Does your laboratory belong to any professional network?

6. Did you copy a similar organization in Greece? Or abroad? Did you use best practices from other organizations? (answers here associated with writing proceedings, documentation, etc.)

7. Was there any motivation or pressure from the decision makers in your university (top management/rectorate)?

8. Did you obtain any external assistance (i.e. consultants or guidance documents) during the implementation of ISO standards?

9. How do you benefit by using ISO standards?

10. How beneficial are the ISO standards of adoption to the university as a whole and in which ways is it connected with the quality of the university?

11. Do you want to add anything else regarding your ISO practice? 


\section{References}

ADIP (2010). Report for the Quality of Higher Education Year 2009. Athens, Greece.

Alstete, W. J. (1995). Benchmarking in Higher Education: Adapting Best practices To Improve Quality. Washington, DC: George Washington University.

Amaral, A. (1998). The US Accreditation System and the CRE's Quality Audits - a Comparative Study. Quality Assurance in Education, Vol. 6(No. 4), pp. 184196.

Angelo, T. (1999). Doing Assessment As If Learning Matters Most. AAHE Bulletin. Antonopoulou, M. (1991). Crisis in the universities. In The University in Greece Today. Athens: Sakis Karagiorgas Foundation.

Asderaki, F. (2009). The Impact of the Bologna Process on the Development of the Greek Quality Assurance System Quality in Higher Education, 15(2), 105122.

Babbie, E. (1990). Survey research methods. Belmont: Wadsworth.

Babbie, E. (2001). The practice of social research. Belmont: Wadsworth/Thomson Learning.

Baldridge, J. V., \& Deal, T. E. (1983). The basics of change in educationla organizations. In J. V. Baldridge, T. E. Deal \& G. L. Ingols (Eds.), The Dynamics of Organizational Change in Education. Berkeley, CA: McCutchan.

Baldridge, J. V., Curtis, D. V., Ecker, G. P., \& Riley, G. L. (1977). Alternative Models of Governance in Higher Education. In G. Riley \& J. V. Baldridge (Eds.), Governing Academic Organizations (pp. 2-25). Berkeley, Ca: McCutchan Publ, Co.

Banta, T. W. (1985). Use of Outcomes Information at the University of Tennessee, Knoxville. New Directions for Institutional Research.

Banta, T. W. (1986). Performance Funding in Higher Education: A Critical Analysis of Tennessee. Boulder, CO: NCHEMS Publications.

Banta, T. W. (1993). Is There Hope for TQM in the Academy? In D. Hubbard (Ed.), Continuous Quality Improvement. Making the Transition to Education (pp. 142-157). Maryville, MO: Prescott

Banta, T. W. (2002). Building a Scholarship of Assessment. San Francisco, CA: JosseyBass.

Banta, T. W. (Ed.). (2003). Portfolio Assessment: Uses, Cases, Scoring, and Impact. San Francisco, CA: Jossey-Bass.

Baran, S. J. (2002). Introduction to Mass Communication: Media Literacy and Culture. Boston: McGraw Hill.

Baruch, Y. (1993). Self performance appraisal vs direct-manager appraisal: A case of congruence. Journal of Managerial Psychology, 11(6), 50-65. 
Belle, R. A. (2000). Benchmarking and enhancing best practices in the engineering and construction sector. Journal of Management in Engineering, 16(4), 58-71.

Benos, S. (2003). O Nomos 1268/82 kai oi metagenesteres rythmiseis gia ta AEI. [Law1268/82 and the Subsequent Regulations for HEIs] (6th ed.). Athens: Benos.

Bensimon, E. M. (1995). Total Quality Management in the Academy: A Rebellious Reading. Harvard Educational Review, 65(4), 593-611.

Berger, P., \& Luckman, T. (1967). The Social Construction of Reality. New York: Doubleday.

Bevans-Gonzales, T., \& Nair, A. (2004). The strengths and weaknesses of ISO 9000 in vocational education Journal of vocational education and training 56(2), 163-180.

Biliris, H. (2004). The National System of Higher Education in Greece: Waiting for a Systematic Quality Assurance System. In S. Schwarz \& D. Westerheijden, F. (Eds.), Accreditation and Evaluation in the European Higher Education Area (pp. 197-206). Dordrecht: Springer.

Birnbaum, R. (Ed.). (1988). How colleges work: the cybernetics of academic organization and leadership. San Francisco: Jossey-Bass.

Birnbaum, R. (2000). Management fads in higher education. San Francisco, CA: Jossey-Bass.

Birnbaum, R. (2001). Management fads in higher education. Where they come from, what they do, and why they fail. San Francisco, CA: Jossey Bass.

Blazey, M., Davison, K. S., \& Evans, J. (2003). Insights to Performance Excellence in Education, 2003: An Inside Look at the 2003 Baldrige Award Criteria for Education Milwaukee, WI: ASQ Quality Press.

Bleiklie, I. (1998). Justifying the Evaluative State: New Public Management Ideals in Higher Education European Journal of Education, 33(3 The Evaluative State Revisited: 20th Anniversary Issue of Review of Trends in Higher Education), 299-316.

de Boer, H. (2003). Who's afraid of red, yellow and blue? The colorful world of management reforms. In A. A. Amaral, V. L. Meek \& I. M. Larsen (Eds.), The higher education managerial revolution? (pp. 89-108). Dordrecht /Boston /London: Kluwer Academic Publishers.

Boiral, O., \& Roy, M. J. (2007). ISO 9000: integration rationales and organizational impacts. International Journal of Operations \& Production Management, 27(2), 226-247.

Bolman, L. G., \& Deal, T. E. (2003). Reframing Organizations - Artistry, Choice an Leadership. San Francisco, CA: Jossey-Bass.

Bologna Declaration. (1999). The European Higher Education Area. Joint Declaration of the European Ministers of Education. Convened in Bologna on the 19th of June 1999 
Bonikos, D. (1998). Do not Change the Rules, Change the Game. In D. Keridis \& C. Sfatos (Eds.), Greek Higher Education. Prospects for Reform (Vol. 1, pp. 8591). New York: Pella Publishing Company, INC.

Bourantas, D., \& Papadakis, V. (1996). Greek Management: Diagnosis and Prognosis. International Studies of Management \& Organization, 26(3), 13-32.

Bourantas, D., Anagnostelis, J., Mantes, Y., \& Kefalas, A. G. (1990). Culture Gap in Greek Management. Organization Studies, 11(2), 261-283.

Bourantas, D., Lioukas, S., \& Papadakis, P. (1996). University Evaluation Systems in Greece: Athens University of Economics and Business, Greece

Boyatzis, R. (1998). Transforming qualitative information: Thematic analysis and code development. Thousand Oaks, California: Sage.

Brennan, J. (1997). Authority, Legitimacy and Change: The Rise of Quality Assessment in Higher Education. Higher Education Management, 9(1), 7-29.

Brennan, J., \& T. Shah (Eds.). (2000). Managing Quality in Higher Education: an international perspective on institutional assessment and change. Buckingham: Open University Press.

Brennan, J., de Vries, P., \& Williams, R. (1997). Standards and Quality in Higher Education. London and Bristol, PA: Jessica Kingsley Publishers.

Burns, J. M. (1978). Leadership. New York: Harper \& Row.

Campbell, C., Kanaan, S., Kehm, B., Mockiene, B., Westerheijden, D. F., \& Williams, R. (2000). The European University: A Handbook on Institutional Approaches to Strategic Management, Quality Management, European Policy and Academic Recognition. Torino: European Training Foundation.

Casile, M., \& Davis-Blake, A. (2002). When accreditation standards change: Factors affecting differential responsiveness of public and private organizations. Academy of Management Journal, 45(1), 180-195.

Chaffee, E. E., \& Sherr, L. A. (Eds.). (1992). Quality: transforming postsecondary education. ASHE-ERIC Higher Education Report No. 3. Washington: George Washington University School of Education and Human Development.

Chadjipantelis, T., \& Papadimitriou, A. (2010). Ranking and Greek Universities

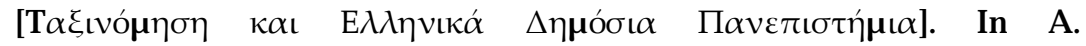
Dimitropoulos, N. Maravejias \& A. Mitsos (Eds.), The University Today: Aspects of the crisis and adjustment steps (pp. 274-301). Athens, Greece: Themelio, EPE.

Chen, C. C., \& Meindl, J. R. (1991). The Construction of Leadership Images in the Popular Press: The Case of Donald Burr and People Express. Administrative Science Quarterly, 36(4), 521-551.

Clark, B. (1970). The distinctive college: Antioch, Reed E Swarthmore. Chicago: Aldine Pub. Co. 
Clark, B. (1983). The Higher Education System. Academic Organization in CrossNational Perspective. Berkeley: University of California Press.

Clark, B. (1997). Small worlds, different worlds: The uniqueness and troubles of American academic professions. Daedalus, 126(4), 21-42.

Clark, B. (Ed.). (1998). Creating entrepreneurial universities: organizational pathways of transformation. Oxford: Pergamon.

Clark, B., \& Neave, G. R. (1992). The Encyclopedia of Higher Education. New York: Pergamon Press.

Clark, Martire, \& Bartolomeo, Inc. (1984). Relating to Readers in the 80's. Charlotte, NC: American Society of Newspaper Editors.

Coate, E. (1990). TQM on Campus. Implementing Total Quality Management in a University Setting. NACUBO Business Officer (November), 26-35.

Cohen, B. (1963). The press, the public and foreign policy. Princeton, NJ: Princeton University Press.

Cohen, M. D., \& March, J. D. (1974). Leadership and Ambiguity: The American College President. New York: McGraw-Hill.

Colling, C., \& Harvey, L. (1995). Quality control, assurance and assessment - the link to continuous improvement. Quality Assurance in Education, 3(4), 3034.

Conyers, J., \& Evy, R. (2004). Charting Your Course Lessons Learned During the Journey Toward Performance Excellence. Milwaukee, Wisconsin: ASQ Quality press.

Cornesky, R. (1991). Implementing Total Quality Management in Higher Education. Madison, WI: Magnar Publications

Creswell, J. W. (1994). Research Design: Quantitative and Qualitative Approaches. Thousand Oaks, CA: Sage.

Creswell, J. W. (2003). Research design: Qualitative, quantitative and mixed methods approaches. (2nd ed.). Thousand Oaks, CA: Sage.

Creswell, J. W. (2005). Educational Research. Planning, Conducting, and Evaluating Quantitative and Qualitative Research (3rd ed.). Upper Saddle River, NJ: Pearson Education, Inc.

Creswell, J. W. (2009). Mapping the Field of Mixed Methods Research. Journal of Mixed Methods Research, 3(2), 95-108.

Creswell, J. W., \& Plano Clark, V. (2007). Designing and Conducting Mixed Methods Research: Sage Publications Inc.

Creswell, J. W., \& Tashakkori, A. (2007). Editorial: Developing Publishable Mixed Methods Manuscripts. Journal of Mixed Methods Research, 1(2), 107-111.

Creswell, J. W., Plano Clark, V. L., Gutmann, M. L., \& Hanson, W. E. (2003). Advanced mixed methods research designs. In A. Tashakkori \& C. Teddlie (Eds.), Handbook of mixed methods in social and behavioral research (pp. 209-240). Thousand Oaks, Ca: Sage. 
Crosby, P. B. (1984). Quality Without Tears: The Art of Hassle-Free Management. Milwaukee: WI: Quality Press.

Csizmadia, T. G. (2006). Quality Management in Hungarian Higher Education: Organizational responses to governmental policy. University of Twente, CHEPS. Enschede, The Netherlands

Csizmadia, T., Enders, J., \& Westerheijden, D. (2008). Quality Management in Hungarian Higher Education: Organizational responses to governmental policy. Higher Education, 56(4), 439-455.

Currall, S. C., \& Towler, A. J. (2003). Research Methods in Management and Organizational Research: Toward Integration of Qualitative and Quantitative Techniques. In A. Tashakkori \& C. Teddlie (Eds.), Handbook of Mixed Methods in Social and Behavioral Research (pp. 513-526). Thousand Oaks, CA: Sage.

Czaja, R., \& Blair, J. (2005). Designing Surveys: A Guide to Decisions and Procedures. Thousand Oaks, CA: Pine Forge Press.

Daft, R. (1992). Organizations: Theory and design (4th ed.). St. Paul, MN: West.

Dale, B. G., Cooper, G. L., \& Wilkinson, A. (1997). Managing Quality \& Human Resources. Oxford: Blackwell.

Davies, J. L. (1997). A European Agenda for Change in Higher Education in the 21 Century: Comparative Analysis of Twenty Institutional Case Studies. Bucharest: joint publication: UNESCO/CEPES, 1997, and CRE-Action 111

Dawkins, E. C. (2005). First to Market: Issue Management Pacesetters and the Pharmaceutical Industry Response to AIDS in Africa. Business and Society, 44(3), 244-282.

DeFleur, M., L., \& Ball-Rokeach, S. (1989). Theories of Mass Communication (5th ed.). New York: Longman.

Dellinger, A. B., \& Leech, N. L. (2007). Toward a Unified Validation Framework in Mixed Methods Research. Journal of Mixed Methods Research, 1(4), 309332.

Delmas, M. A. (2002). The diffusion of environmental management standards in Europe and in the United States: An institutional perspective. Policy Sciences, 35(1), 91-119.

Dervitsiotis, K., N. (1999). Quality in Greece: past and present. TQM Magazine, 11(2), 84-87.

Dill, D. (1992). Quality by Design: Towards a Framework for Academic quality management. In J. Smart (Ed.), Higher Education: handbook of Theory and Research (Vol. VIII, pp. 37-83). New York: Agathon Press.

Dill, D. (1995). Through Deming's Eyes: a cross-national analysis of quality assurance policies in higher education. Quality in Higher Education, 1(2), 95-110. 
Dill, D. (1999). Academic accountability and university adaptation: The architecture of an academic learning organization. Higher Education, 38(2), 127-154.

Dill, D. (2003). An institutional perspective on Higher Education Policy: the case of academic quality assurance. In J. C. Smart (Ed.), Higher education: handbook of theory and research, (Vol. XVIII, pp. 669-700): Agathon Press New York.

Dillman, D. A. (2000). Mail and Internet Surveys: The Tailored Design Method (2nd ed.). New York, NY: Willey.

DiMaggio, P. J., \& Powell, W. W. (1983). The Iron Cage Revisited: Institutional Isomorphism and Collective Rationality in Organizational Fields. American Sociological Review, 48(2), 147-160.

DiMaggio, P. J., \& Powell, W. W. (1991). The Iron Cage Revisited: Institutional Isomorphism and Collective Rationality in Organizational Fields. In W. W. Powell \& P. J. DiMaggio (Eds.), The New Institutionalism in Organizational Analysis (pp. 63-83). Chicago: University of Chicago Press.

Dimmick, J. (1974). The gate-keeper: An uncertainty theory. Journalism Monographs, 37, 1-39.

Dinas, E., \& Gemenis, K. (2007). Rebels against their cause? Mobilization and political participations among Greek university students. Paper presented at the 4 th ECPR General Conference.

Drucker, P. (1992). The New Society of Organizations. Harvard Business Review, Sept-Oct. 95-104.

Drucker, P. (1995). Managing in a Time of Great Change. New York: Truman Talley Books/Dutton.

EC (European Commission) (2003). Third European Report on Science E Technology Indicators. Brussels: European Commission, Directorate General for Research.

Edwards, D. (1991). Total Quality Management in Higher Education. Management Services, 35(12), 18-20.

Eleftheriou, A., \& Robertson, I. (1999). A Survey of Management Selection Practices in Greece. International Journal of Selection and Assessment, 7(4), 203-208.

Eliou, M. (1992). Greece. In B. R. Clark \& G. R. Neave (Eds.), The Encyclopedia of Higher Education (Vol. 1, pp. 265-275). New York: Pergamon Press.

Elsbach, K. D., \& Sutton, R. I. (1992). Acquiring organizational legitimacy through illegitimate actions: A marriage of institutional and impression management theories. Academy of Management Journal, 35(4), 699-738.

Empson, L. (2006). Professionals in partnership. In C. John (Ed.), Production Values: Futures of Professionalism (pp. 144-152). London, UK: Demos. 
Enders, J. (2004). Higher education, internationalisation, and the nation-state: Recent developments and challenges to governance theory. Higher Education, 47(3), 361-382.

Erden, Z. (2006). Histories, Institutional Regimes and Educational Organizations: The case of Turkish Higher Education. Unpublished doctoral Dissertation. Sabancı University. Istanbul.

Ewell, P. T. (1991). Assessment and TQM: In search of convergence. In L. A. Sherr \& D. J. Teeter (Eds.), Total quality management in higher education (pp. 3952). San Francisco: Jossey-Bass.

Ewell, P. T. (2005). Can assessment serve accountability? It depends on the question. In J. Burke \& Associates (Eds.), Achieving accountability in higher education (pp. 1-24). San Francisco, CA: Jossey-Bass.

Fetterman, D. (1998). Ethnography (2nd ed. Vol. 17). Thousand Oaks, CA: Sage.

Fink, A. (1995). How to Analyze Survey Data. Thousand Oaks, CA: Sage.

Ford, M. W., \& Evans, J. (2000). Conceptual Foundations of Strategic Planning in the Malcolm Baldrige Criteria for Performance Excellence. Quality Management Journal, 7(1), 8-26.

Fowler, F. J. (1993). Survey research methods (2nd ed.). Newbury Park: Sage Publications.

Frooman, J. (1999). Stakeholder influence strategies. Academy of Management Review, 24(2), 191-205.

Gaither, G. (1998). The Future Dynamics of Quality Assurance: Promises and Pitfalls. New Directions for Institutional Research, 1998(99), 87-91.

Garvin, D. A. (1991). How the Baldrige Award really works. Harvard Business Review (November- December), 80-95.

Gioia, D. A., \& Thomas, J. B. (1996). Identity, Image, and Issue Interpretation: Sensemaking During Strategic Change in Academia. Administrative Science Quarterly, 41(3), 370-403.

Gornitzka, A. (1999). Governmental policies and organizational change in higher education. Higher Education, 38(1), 5-31.

Gornitzka, A., \& Maassen, P. (2000). Analyzing organizational change in higher education. In F. Engelstad (Ed.), Comparative Perspectives on Universities (Vol. 19, pp. 83-99): Emerald Group Publishing Limited.

Gornitzka, A., \& Maassen, P. (2000). Hybrid steering approaches with respect to European higher education. Higher Education Policy, 13(3), 267-285.

Gornitzka, A., Kyvik, S., \& Stensaker, B. (2005). Implementation Analysis in Higher Education. In A. Gornitzka, M. Kogan \& A. Amaral (Eds.), Reform and Change in Higher Education (pp. 35-56). Dordrecht; Boston; London: Springer. 
Gotzamani, K., \& Tsiotras, G. (2001). An empirical study of the ISO 9000 standards' contribution towards total quality management. International Journal of Operations \& Production Management, 21(10), 1326-1342.

Grant, D., Mergen, E., \& Widrick, S. (2004). A Comparative Analysis of Quality Management in US and International Universities. Total Quality Management $\mathcal{E}$ Business Excellence, 15(4), 423 - 438.

Green, D. (1994). What Is Quality in Higher Education? Buckingham, Open University press and Society for Research into Higher Education.

Green, K., \& Gilbert, S. (1995). Great expectations: Content, communications, productivity, and the role of information technology in higher education. Change, 27(2), 8-18.

Green, M. F. (1997). Transforming higher education: views from leaders around the world. Washington, DC: American Council on Education.

Greene, J. C. (2007). Mixed Methods in social inquiry. San Francisco: Jossey-Bass.

Greene, J. C., Caracelli, V. J., \& Graham, W. F. (1989). Toward a Conceptual Framework for Mixed-Method Evaluation Designs. Educational Evaluation and Policy Analysis, 11(3), 255-274.

Greening, D. W., \& Gray, B. (1994). Testing a Model of Organizational Response to Social and Political Issues. The Academy of Management Journal, 37(3), 467-498.

Greenwood, R., \& Meyer, R. (2008). Influencing Ideas. A celebration of DiMaggio and Powell, (1983). Management Inquiry, 17(4), 258-264.

Guler, I., Guillén, M. F., \& Macpherson, J. M. (2002). Global Competition, Institutions, and the Diffusion of Organizational Practices: The International Spread of ISO 9000 Quality Certificates. Administrative Science Quarterly, 47(2), 207-232.

Gumport, P. J., \& Sporn, B. (1999). Institutional Adaptation: Demands for Management Reform and University Administration. In J. Smart (Ed.), Higher Education: Handbook of Theory and Research (Vol. XIV). New York, NY: Agathon.

Gunther, A. C. (1992). Biased press or biased public? Attitudes toward media coverage of social groups. Public Opinion Quarterly, 56(2), 147-167.

Hall, R. (1999). Organizations: Structures, Processes, and Outcomes (7th ed.). Upper Saddle River, NJ: Prentice-Hall, Inc.

Harris, L. (1983). Address before the forty-ninth annual convention of the Associated Press Managing Editors. Louisville, KY.

Harris, P., Kolovos, I., \& Lock, A. (2001). Who sets the agenda? An analysis of agenda setting and press coverage in the 1999 Greek European elections. European Journal of Marketing, 9(10), 1117-1135.

Harrison, M. I. (1994). Diagnosing Organizations. Methods, Models, and Processes. Thousand Oaks, CA: Sage. 
Harvey, L. (1998). An Assessment of Past and Current Approaches to Quality in Higher Education. Australian Journal of Education, 42(3), 237-238.

Harvey, L., \& Green, D. (1993). Defining Quality. Assessment \& Evaluation in Higher Education, 18(1), 9 - 34.

Hatch, A. J. (2002). Doing Qualitative Research in Education Settings. Albany, NY: SUNY Press.

Haveman, H. A. (1993). Follow the Leader: Mimetic Isomorphism and Entry into New Markets. Administrative Science Quarterly, 38(4), 593-627.

Haveman, H. A., \& David, R., J. (2008). Ecologists and Institutionalists: Friends or Foes? In R. Greenwood, C. Oliver, R. Suddaby \& K. Sahlin (Eds.), Handbook of Organizational Institutionalism (pp 573-595). Thousand Oaks, CA: Sage.

Hofmann, S. (2005). 10 years on lessons learned from the Institutional Evaluation Programme. Brussels: European University Association.

Horine, J., \& Hailey, W. A. (1995). Challenges to successful quality management implementation in higher education institutions. Innovative Higher Education, 20(1), 7-17.

Hoyle, D. (2003). ISO 9000:2000 An A-Z Guide. Oxford, UK: ButterworthHeinemann.

Huisman, J., \& van der Wende, M. (2004). The EU and Bologna: are supra- and international initiatives threatening domestic agendas. European Journal of Education, 39(3), 349-356.

Huisman, J., \& van Vught, F. (2009). Diversity in European Higher Education: Historical Trends and Current Policies. In F. van Vught (Ed.), Mapping the Higher Education Landscape, (Vol. 28, pp. 17-37): Springer Science and Business Media B.V.

Huisman, J., Stensaker, B., \& Kehm, B. (2009). Bologna, Quo Vadis? In B. Kehm, J. Huisman \& B. Stensaker (Eds.), The European Higher Education Area: Perspectives on a Moving Target (pp. xiii-xx). Rotterdam: Sense.

Hwang, H., \& Powell, W. W (2009). The Rationalization of Charity: The influences of Professionalism in the Nonprofit Sector. Administrative Science Quarterly, 54, 268-298.

Izadi, M., Kashef, A. E., \& Stadt, R. W. (1996). Quality in Higher Education: Lessons Learned from the Baldrige Award, Deming Prize, and ISO 9000 Registration Journal of Industrial Teacher Education, 33(2), 60-76.

Jeliazkova, M., \& Westerheijden, D. F. (2002). Systemic adaptation to a changing environment: Towards a next generation of quality assurance models. Higher Education, 44(3), 433-448.

Jelinek, S., Foster, R., Jr., \& Sauser, W. J. (1995). A Rose by Any Other Name: Applying Total Quality Management to Higher Education. In S. Sims \& 
R. Sims (Eds.), Total Quality Management in Higher Education. Is It Working? Why or Why Not? (pp. 107-126). Westport, CT: Praeger.

Johnson, M. E., Hanna, D. E., \& Olcott, D. (2003). Bridging the gap: leadership, technology, and organizational change for university deans and chairpersons. Madison, Wis.: Atwood Pub.

Johnson, R. B., \& Onwuegbuzie, A. J. (2004). Mixed Methods Research: A Research Paradigm Whose Time Has Come. Educational Researcher, 33(7), 14-26.

Johnson, R. B., Onwuegbuzie, A. J., \& Turner, L. A. (2007). Toward a Definition of Mixed Methods Research. Journal of Mixed Methods Research, 1(2), 112-133.

Karapetrovic, S. (2002). Why and How to Develop a Meaningful Quality Assurance System in Engineering Schools. International Journal of Engineering Education, 18(3), 285-294.

Karmas, C. A., Lianos, T. P., \& Kalamatianou, A. G. (1988). Greek Universities: An Overview European Journal of Education, 23(3), 261-269.

Katsikas, S., Papazoglou, V., \& Tsakloglou, P. (2008). Quality Assurance in Greek Universities: Challenges and Prospects, paper presented at the Conference "Where the Greek University goes?" Athens, Greece.

Katz, D., \& Kahn, R. (1978). The social psychology of organizations (2nd ed.). New York, NY: Wiley.

Keller, G. (1983). Academic Strategy: The Management Revolution in American Higher Education Baltimore: Johns Hopkins University Press.

Kendall, J. (2005). Extent of congruence between student perception and media representations of quality of higher education institutions. Unpublished Doctoral dissertation, University of Oklahoma.

Keridis, D., \& Sfatos, C. (1998). Greek Higher Education: The Challenge of Reform. In D. Keridis \& C. Sfatos (Eds.), Greek Higher Education: Prospects for Reform (Vol. 1, pp. 173-179). New York, NY: Pella Publishing Company, INC.

Kerlinger, F. N. (1973). Foundations of Behavioral Research. New York: Holt, Rinehart, \& Winston.

Kerlinger, F. N., \& Lee, H. B. (2000). Foundations of Behavioral Research (4th ed.). Orlando, FL: Harcourt.

Kerr, C. (2001). The Use of the University (5th ed.). Cambridge, MA: Harvard University Press.

Kingdon, W. J. (1995). Agendas, Alternatives, and Public Policies (2nd ed.). New York, NY: HarperCollins College Publishers.

Kladis, D. (2008). Experiences of self-evaluation: The case of Greece. Paper presented at the Conference on self-evaluation of higher education institutions. Belgrade, Serbia. 
Kogan, M., \& S. Hanney (Eds.). (2000). Reforming higher education. London: Jessica Kingsley.

Kokosalakis, N. (2001). Lifelong Learning in Greek Universities: policies, practices and prospects. European Journal of Education, 36(3), 329-339.

Koopman, P. L., Hartog, D. N. D., Konrad, E., et al. (1999). National Culture and Leadership Profiles in Europe: Some Results from the GLOBE Study. European Journal of Work E Organizational Psychology, 8(4), 503-520.

Koufteros, X. A., \& Vonderembse, M. A. (1998). The impact of organizational structure on the level of JIT attainment: theory development. International Journal of Production Research, 36(10), 2863-2878.

Kraatz, M. S., \& Moore, J. H. (2002). Executive Migration and Institutional Change. Academy of Management Journal, 45(1), 120-143.

Kraut, A. (1996). Organizational Surveys: Tools for Assessment and Change. San Francisco, CA: Jossey-Bash Inc. Publishers.

Krippendorff, K. (1980). Content Analysis: An Introduction to Its Methodology. Newbury Park, CA: Sage.

Kristoffersen, D., Sursock, A., \& Westerheijden, D. F. (Eds.). (1998). Manual of Quality Assurance: Procedures and Practice. Torino: European Training Foundation.

Krücken, G. (2007). Organizational Fields and Competitive Groups in Higher Education: Some Lessons from the Bachelor/Master Reform in Germany. Management Review, 18(2), 187-203.

Kumar, M. R. (2007). Comparison between DP and MBNQA: convergence and divergence over time. The TQM Magazine, 19(3), 245-258.

Kyriazis, A. (2006). Hellenic Higher Education System and its Reforms. Paper presented at the OECD meeting, Athens, July 2006, Greece.

Kyriazis, A. (2007). Standards and Guidelines for Quality Assurance Eletherotipia ( $15 / 7 / 2007)$

Kyriazis, A., \& Asderaki, F. (2008). Higher Education in Greece. Bucharest: UNESCO-CEPES.

Lang, K., \& Lang, G. E. (1966). The mass media and voting. In B. B. Berelson \& M. Janowitz (Eds.), Reader in Public Opinion and Communication (2nd ed). New York: Free Press.

Larson, C. U. (1986). Persuasion (4th ed). Belmont, CA: Whadswort.

Laurent, A. (1986). The cross-cultural puzzle of international human resource management. Human Resource Management, 25(1), 91-102.

Law 3374/2005. (2005). Quality Assurance in Higher Education, Establishment of the European Credit Transfer and Accumulation System and Diploma Supplement. Official Journal of the Hellenic Republic 189/2-8-2005, Athens, Greece. 
Law 3549/2007. (2007). Reform of the legal framework for the structure and the operation of universities. Official Journal of the Hellenic Republic 69A/2007, Athens, Greece.

Leblebici, H., Salancik, G. R., Copay, A., \& King, T. (1991). Institutional Change and the Transformation of Interorganizational Fields: An Organizational History of the U.S. Radio Broadcasting Industry. Administrative Science Quarterly, 36(3), 333-363.

Leicht, K. T., \& Fennell, L. M. (2008). Institutionalism and the Professions. In R. Greenwood, C. Oliver, R. Suddaby \& K. Sahlin (Eds.), The SAGE Hanbook of Organizational Insitutionalism (pp. 431-448). Los Angeles: Sage.

Lewis, R. G., \& Smith, D. H. (1994). Total Quality in Higher Education. Delray Beach, FL: St Lucie Press.

Lincoln, Y., \& Guba, E. (1985). Naturalistic inquiry. New York, NY: Sage.

Lipovatz, D., Stenos, F., \& Vaka, A. (1999). Implementation of ISO 9000 quality systems in Greek enterprises. International Journal of Quality $\mathcal{E}$ Reliability Management, 16(6), 534-551.

Lippmann, W. (1922). Public opinion. New York: Macmillan (reprint, 1965)Free Press.

Loomba, A. P. S., \& Johannessen, T. B. (1997). Malcolm Baldrige National Quality Award: Critical issues and inherent values. Benchmarking and International Journal, 4(1), 59-77.

Louloudis, L. (2010). The 'underfunding' of universities; yes, maybe, maybe not? In A. Dimitropoulos, N. Maravejias \& A. Mitsos (Eds.), The University Today: Aspects of the crisis and adjustment steps (pp. 148-171). Athens, Greece: Themelio, EPE

Lounsbury, M. (2001). Institutional sources of practice variation: Staffing college and university recycling programs. Administrative Science Quarterly, 46, 29-56.

Loya, T. A., \& Boli, J. (1999). Standardization in the world polity: Technical rationality over power. In J. J. Boli \& G. M. Thomas (Eds.), Constructing world culture: International nongovernmental organizations since 1875 (pp. 169-197). Stanford, CA: Stanford University Press.

Lundqvist, R. (1997). Quality System and ISO 9000 in Higher Education. Assessment E Evaluation in Higher Education, 22(2), 159-172.

Maassen, P. A. M., \& Potman, H. P. (1990). Strategic decision making in higher education. An analysis of the new planning system in Dutch higher education. Higher Education, 20(4), 393-410.

Maassen, P., \& Stensaker, B. (2005). The Black Box Revisited; the Relevance of Theory-Driven Research in the Field of Higher Education Studies. In I. Bleiklie \& M. Henkel (Eds.), Governing Knowledge (pp. 213-226). Dordrecht: Springer. 
Makridakis, S., Caloghirou, Y., Papagiannakis, L., \& Trivellas, P. (1997). The dualism of Greek firms and management: Present state and future implications. European Management Journal, 15(4), 381-402.

Mangione, T. (1995). Mail Surveys: Improving the Quality (Vol. 40). Thousands Oaks, CA: Sage.

March, J. D. (1988). Decisions and Organizations. New York, NY: Blackwell.

Marseilles, M. (1996). Funding by numbers provokes Greek university fury. Times Higher Education Supplement (5 August 1996).

Massy, W. F. (2003). Honoring the trust: quality and cost containment. Bolton: Anker Publishing Company.

McCombs, M. E., \& Washington, L. (1983). Opinion Surveys Offer Conflicting Cues as to How Public Views Press. Presstime, 5(2), 4-9.

McCombs, M., Esteban López-Escobar, \& Llamas, J. P. (2000). Setting the Agenda of Attributes in the 1996 Spanish General Election. Journal of Communication, 50(2), 77-92.

Merriam, S. B. (1998). Qualitative research and case study applications in education. San Francisco, CA: Jossey-Bass.

Merton, R. K., Fiske, M., \& Kendall, P.L. (1990). The focused interview: A manual of problems and procedures (2nd ed.). London: Collier MacMillan.

Meyer, J. W., \& Rowan, B. (1977). Institutionalized Organizations: Formal Structure as Myth and Ceremony. The American Journal of Sociology, 83(2), 340-363.

Meyer, J. W., \& Rowan, B. (1991). Institutionalized Organizations: Formal Structure as Myth and Ceremony. In W. Powell \& P. J. DiMaggio (Eds.), The New Institutionalism in Organizational Analysis (pp. 41-63). Chicago, IL: The University of Chicago Press.

Miles, M. B., \& Huberman, A. M. (1994). Qualitative Data Analysis: An Expanded Sourcebook. London \& Thousand Oaks, California: Sage.

Mintzberg, H. (1979). The structuring of organizations: a synthesis of the research. Engewood Cliffs, NJ: Prestigge-Hall Inc.

Mintzberg, H. (1983). Structures in Five. Englewood Cliffs, NJ: Prestigge-Hall Inc.

Mizruchi, M. S., \& Fein, L. C. (1999). The social construction of organizational knowledge: A study of the uses of coercive, mimetic, and normative isomorphism. Administrative Science Quarterly, 44(4), 653-683.

Moore, N. (1996). Using the Malcolm Baldrige Criteria to improve quality in higher education. Paper presented at the Forum of the Association of Institutional Research.

Moreland, N., \& Clark, M. (1998). Quality and ISO 9000 in educational organizations. Total Quality Management, 9(2/), 311-320.

Morgan, G. (1986). Images of Organizations. Newbury Park, CA: Sage. 
Morgan, G. (1998). Images of Organizations: The Executive Edition. Thousand Oaks, CA: Sage.

Morse, J. (2003). Principles of Mixed Methods and Multimethod Research Design. In A. Tashakkori \& C. Teddlie (Eds.), Handbook of Mixed Methods in Social $\mathcal{E}$ Behavioral Research (pp. 189-208). Thousand Oaks: Sage.

Musselin, C. (2009). The Side Effect of the Bologna Process on National Institutional Settings: The Case of France. In A. Amaral, G. Neave, C. Musselin \& P. Maassen (Eds.), European Integration and the Governance of Higher Education and Research (pp. 207-226). New York, NY: Springer.

Myloni, B., Harzing, A.-W., \& Mirza, H. (2004). Human Resource Management in Greece: Have the Colours of Culture Faded Away? International Journal of Cross Cultural Management, 4(1), 59-76.

Nadler, D., \& Tushman, M. (1989). A model for diagnosing organizational behavior. Organizational Dynamics, 9, 35-51.

Nakos, G., E., \& Hajidimitriou, Y., A. (2009). Conducting business in Greece: A brief for international managers. Global Business and Organizational Excellence, 28(5), 70-83.

Neave, G. (1988). On the cultivation of quality, efficiency and enterprise: an overview of recent trends in higher education in Western Europe, 19861988. European Journal of Education, 23(1/2), 7-23.

Neave, G. (1996). Higher education policy as an exercise in contemporary history. Higher Education, 32(4), 403-415.

Neave, G. (Ed.). (1995). The core functions of government: six European perspectives on a shifting educational landscape. Utrecht: National Advisory Council for Education ARO.

Nelson, D. (2004). Agenda-setting and gatekeeping functions in higher education: An analysis of the coverage of two state institutions by the state's two metropolitan newspapers. Unpublished doctoral dissertation, Oklahoma State University.

Newton, J. (2002). Barriers to effective quality management and leadership: Case study of two academic departments. Higher Education, 44(2), 185-212.

Nilsson, J. S., Eaton, J., Marçal-Grilo, E., Scheele, K., \& Henkel, M. (2002). External evaluation of the EUA Institutional Review Programme 1994-2001: Report of the External Review Panel. Brüssel: EUA

NIST (2005). National Institute for Standards and Technology. Baldrige National Quality Program. Education Criteria for Performance Excellence. Retrieved April 15 2005, from

http://www.baldrige.nist.gov/Education_Criteria.htm.

Nygaart, C., \& Kristensen, B. (2009). Quality Work at Copenhagen Business School - how to create a university quality culture. In E. Froment, J. 
Kohler, L. Purser \& L. Wilson (Eds.), EUA Bologna Handbook: Making Bologna work.

O'Cathain, A., Murphy, E., \& Nicholl, J. (2008). The quality of mixed methods studies in health services research. Health Service and Research Policy, 13(2), 92-98.

OECD. (2006). Education at a Glance. OECD Indicators 2006. Paris:OECD

Olie, R. (1995). "The 'culture' factor in personnel and organization policies". In A. W. Harzing \& J. V. Ruysselveld (Eds.), International Human Resource Management. London: Sage.

Oliver, C. (1991). Strategic Responses to Institutional Processes. The Academy of Management Review, 16(1), 145-179.

Oliver, C. (1997). Sustainable competitive advantage: Combining institutional and resource-based views. Strategic Management Journal, 18(697-713).

Omar, P.L. \& Liuhanen, A.M. (2005). A Comparative Analysis of Systematic Quality work in Nordic Higher Education Institutions Helsinki, FN: Nordic Quality Assurance Network in Higher Education.

Onwuegbuzie, A. J., \& Johnson, R. B. (2006). The validity issue in mixed research. Research in the Schools, 13(1), 48-63.

Onwuegbuzie, A. J., \& Teddlie, C. (2003). A framework for analyzing data in mixed methods research. In A. Tashakkori \& C. Teddlie (Eds.), Handbook of mixed methods in social and behavioral research (pp. 351-383). Thousand Oaks, CA: Sage.

Pagano, A., \& Verdin, J. (1997). The External Environment of Business: Political, Economics, Social and Regulatory (2nd ed.). Campaign, IL: Stipes.

Palmer, D., Biggart, N., \& Dick, B. (2008). Is the New Institutionalism a Theory? In R. Greenwood, C. Oliver, R. Suddaby \& K. Sahlin (Eds.), The SAGE Handbook of Organizational Institutionalism (pp. 739-768). Los Angeles: Sage.

Papadimitriou, A. (2004). The Greek Higher Education System: A short overview of Aristotle University and University of Macedonia. Paper presented at the ASHE 29th Annual Conference, International Forum, Kansas, USA

Papadimitriou, A. (2005). International students in Greek Universities. Paper presented at the NASPA International Symposium, Tampa, USA.

Papadimitriou, A. (2009). Motivating Freshman Students in a Business Management Course via Portfolios: Practice from a Greek Public University. Assessment Update, 21(1), 10-12.

Papadimitriou A., \& Tsiotras, G. (2007). MBNQA best practice for educational quality improvement. In D. Papadopoulos (Ed.), Volume of Essays in Honor of Professor Aristoklis Ignatiadis (pp. 853-870). Thessaloniki: University of Macedonia, Greece. 
Papadimitriou, A., \& Ursin, J. (2010). Higher education policies and their effects on universities: the cases of quality reform in Greece and university mergers in Finland. Paper presented at the 23rd CHER Annual Conference, Oslo, Norway.

Papadimitriou, A., \& Westerheijden D.F. (2008). ISO in Greek universities and neoinstitutional theory: relationship through mixed method analysis. Paper presented at the Mixed Methods Conference, Cambridge, UK.

Papadimitriou, A., \& Westerheijden, D. F. (2009). Greek quality assurance challenges beyond 2010. Paper presented at the 4th European Quality Assurance Forum, Copenhagen, Denmark.

Papadimitriou, A., Ursin, J., Westerheijden, D. F., \& Valimaa, J. (2008). Views on Excellence in Finnish and Greek Universities. Paper presented at the 21st CHER Conference, Pavia, Italy.

Papalexandris, N., \& Chalikias, C. (2002). Changes in Training, performance management and communication issues among Greek firms in the 1990s: Intrercountry and Intracountry comparison. Journal of European Industrial Training, 26(7), 342-352.

Papandreou, G. (1998). Administration, Mission and sociopolitical issues: Excerpts from the discussion. In D. Keridis \& C. Sfatos (Eds.), Greek Higher Education. Prospects for Reform (Vol. 1, pp. 141-153). New York: Pella Publishing Company, INC.

Papandreou, G. (1998). Toward the 21st Century: Greek Education in Transition. In D. Keridis \& C. Sfatos (Eds.), Greek Higher Education. Prospects for Reform (Vol. 1, pp. 19-26). New York, NY: Pella Publishing Company, INC.

Patton, M. Q. (1990). Qualitative evaluation and research methods (2nd ed.). Newburry Park, CA: Sage.

Patton, M. Q. (2002). Qualitative research and evaluation methods (3rd ed.). Thousand Oaks, CA: Sage.

Pesmatzoglou, S. (1994). Government, Ideology and the University Curriculum in Greece. European Journal of Education, 29(3), 291-304.

Peters, J. (1999). Educational Accreditation through ISO 9000. Quality Assurance in Education, 7(2), 85-89.

Pettigrew, A. (1977). Strategy Formulation as a Political Process. International Studies in Management and Organization, 7(2), 78-87.

Plano Clark, V. (2005). Cross-disciplinary analysis of the use of mixed methods in physics education research, counselling psychology, and primary care. Unpublished Doctoral Dissertation, Abstract International, 66, 02A, University of Nebraska-Lincoln. 
Plano Clark, V., Leslie-Pelecky, D., \& Garrett, A. (2008). Illustrations of three Strategies for Merging Quantitative and Qualitative Data in Mixed Methods Research. Paper presented at the AERA Conference, New York, USA.

Politis, Y., \& Siskos, Y. (2004). Multicriteria methodology for the evaluation of a Greek engineering department. European Journal of Operational Research, 156(1), 223-240.

Pollitt, C. (2003). Public Management Reform: Reliable Knowledge and International Experience. OECD Journal on Budgeting, 3(3), 121-136.

Pollitt, C., \& Bouckaert, G. (Eds.). (1995). Quality improvement in European public services: concepts, cases and commentary. London: Sage.

Pollitt, C., \& Bouckaert, G. (2000). Public Management Reform: A Comparative Analysis. Oxford: Oxford University Press.

Powell, W. W., \& DiMaggio, P. J. (1991). The New Institutionalism in Organizational Analysis. Chicago: University of Chicago Press.

Psacharopoulos, G. (1988). Efficiency and equity in Greek higher education. Minerva, 26(2), 119-137.

Psacharopoulos, G. (2002). Liberalism and Education. In C. Zachopoulos (Ed.), About Liberalism. Athens: I. Sideris.

Psacharopoulos, G. (2003). The Social Cost of an Outdated Law: Article 16 of the Greek Constitution. European Journal of Law and Economics, 16(2), 123-137.

Psychogios, A. G., \& Priporas, C. V. (2007). Understanding Total Quality Management in Context: Qualitative Research on Managers' Awareness of TQM Aspects in the Greek Service Industry. The Qualitative Report, 12(1), 40-66.

R\&D (2003). Research and Development in Greece. Ministry of Development-General Secretariat for Research and Technology, Athens, Greece.

Radaelli, C. M. (2000). Policy Transfer in the European Union: Institutional Isomorphism as a Source of Legitimacy. Governance, 13(1), 25-43.

Rao Tummala, V. M., \& Tang, C. L. (1996). Strategic quality management, Malcolm Baldrige and European Quality Awards and ISO 9000 certification: core concepts and comparative analysis. International Journal of Quality \& Reliability Management, 13(4), 8-38.

Ravinet, P. (2008). From Voluntary Participation to Monitored Coordination: why European countries feel increasingly bound by their commitment to the Bologna Process. European Journal of Education, 43(3), 353-367.

Rhoades, G. (1992). Organization Theory. In B. R. Clark \& G. R. Neave (Eds.), The Encyclopedia of Higher Education (Vol. 3, pp. 1884-1896). New York: Pergamon Press.

Rhoades, G., \& Sporn, B. (2002). Quality assurance in Europe and the U.S.: Professional and political economic framing of higher education policy. Higher Education, 43(3), 355 - 390. 
Rosa, M. J., \& Amaral, A. (2007). A Self-assessment of Higher Education Institutions from the Perspective of the EFQM Excellence Model. In D.F. Westerheijden, B. Stensaker, \& M. J. Rosa (Eds.), Quality Assurance in Higher Education: Trends in Regulation, Translation and Transformation (pp.181-207). Dordrecht: Springer.

Rosa, M. J., \& Amaral, A. (2008). Evaluation Reports: Do the contribute to quality improvement? In A. Amaral, A. Rovio-Johansson, M. J. Rosa \& D. Westerheijden (Eds.), Essays in Supportive Peer Review (pp. 69-82). New York, NY: Nova Science Publishers.

Rosa, M. J., Tavares, D., \& Amaral, A. (2006). Institutional Consequences of Quality Assessment. Quality in Higher Education, 12(2), 145 - 159.

Rossman, G. B., \& Wilson, B. L. (1985). Number and words: Combining quantitative and qualitative methods in a single large-scale evaluation study. Evaluation Review, 9(5), 627-643.

Rourke, L., Anderson, T. A., Garrison, D. R., \& Archer, W. (2001). Methodological Issues in the Content Analysis of Computer Conference Transcripts. International Journal of Artificial Intelligence in Education, 12(1), 8-22.

Rovio-Johansson, A., Amaral, A., \& Westerheijden, D. F. (2008). Audits As Supportive Peer Reviews. In A. Amaral, A. Rovio-Johansson, D. F. Westerheijden \& M. J. Rosa (Eds.), Essays in Supportive Peer Review (pp. 51-63). New York, NY: Nova Science Publishers.

Ruben, B. D. (1995). Quality in Higher Education. New Brunswick, NJ: Transaction.

Ruben, B. D. (2004). Pursuing Excellence in Higher Education: eight fundamental challenges. San Francisco: Jossey-Bass.

Ruben, B. D. (2005). Excellence in Higher Education: Workbook and Scoring Instructions. An Integrated Approach to Assessment, Planning, and Improving in Colleges and Universities. Washington, DC: NACUBO.

Ruben, B. D. (2006). Departmental Effectiveness: What is it? Why is it Important? How Can It Be achieved? Effective Practices for Academic Leaders, 1(12), 115.

Ruben, B. D. (2007). Higher education assessment: Linking accreditation standards and the Malcolm Baldrige criteria. New Directions for Higher Education, 2007(137), 59-83.

Ruben, B. D., Lehr, J., \& DeAngelis, J. (1999). The value of the Baldrige framework for self-assessment and improvement in education: the Rutgers Excellence in Higher Education Program. Paper presented at the National Quality Education Conference.

Saitis, C. (1988). The Relationship between the State and the University in Greece. European Journal of Education, 23(3), 249-260

Salant, P., \& Dillman, D. A. (1994). How to conduct your own survey. New York, NY: John Wiley and Sons. 
Sallis, E. (1994). From systems to leadership: the development of the quality movement in higher education. In G. Doherty (Ed.), Developing quality systems in education. London: Routledge.

Schneider, S. C. (1989). Strategy Formulation: The Impact of National Culture. Organization Studies, 10(2), 149-168.

Schwarz, S., \& Westerheijden, D. F. (2004). Accreditation in the Framework of Evaluation Activities: A Comparative Study in the European Higher Education Area. In S. Schwarz \& D. F. Westerheijden (Eds.), Accreditation and Evaluation in the European Higher Education Area (pp. 1-41). Dordrecht; Boston; London: Kluwer Academic Publishers.

Schwarz, S., \& Westerheijden, D., F. (Eds). (2004). Accreditation and Evaluation in the European Higher Education Arena. Dordrecht; Boston; London: Kluwer Academic Publishers.

Scott, W. R. (1981). Organizations: Rational, Natural, and Open Systems. Englewood Cliffs: Prentice-Hall.

Scott, W. R. (1987). The Adolescence of Institutional Theory. Administrative Science Quarterly, 32(4), 493-511.

Scott, W. R. (1991). Unpacking institutional arguments. In W. W. Powell \& P. J. DiMaggio (Eds.), The new institutionalism in organizational analysis (pp. 164-182). Chicago: University of Chicago Press.

Scott, W. R. (1995). Institutions and Organizations. Thousand Oaks, CA: Sage Publications.

Scott, W. R. (1995). Introduction: institutional theory and organizations. In W. R. Scott \& S. Christensen (Eds.), The Institutional Construction of Organizations: International and Longitudinal studies (pp. xi-xxiii). Thousand Oaks, CA: Sage.

Scott, W. R. (2003). Organizations: Rational Natural and Open Systems (5th ed.). Upper Saddle River, NJ: Prentice Hall.

Scott, W. R. (2008). Institutions and Organizations (3rd ed.). Thousand Oaks, CA: Sage.

Senge, P. (1990). The Fifth Discipline: The Art and Practice of the Learning Organization. New York, NY: Doubleday Currency.

Seymour, D. (1995). TQM: Focus on Performance, Not Resources. In B. D. Ruben (Ed), Quality in Higher Education (pp. 145-157). New Brunswick, NJ: Transaction.

Seymour, D. T. (1991). On Q: Causing Quality in Higher Education. New York: ACE MacMillian.

Seymour, D. T. (1996). The Baldrige in education: Why is it needed, and what the first year pilot produced. AAHE Bulletin, 48(8), 9-14. 
Seymour, D., \& Collett, C. (1991). Total Quality Management in Higher Education: A Critical Assessment (Report No 91-01). Methuen, MA: GOAL/QPC.

Sherer, P. D., \& Lee, K. (2002). Institutional change in large law firms: A resource dependency and institutional perspective. Academy of Management Journal, 45(1), 102-119.

Sherr, L. A., \& Lozier, G. G. (1991). Total Quality Management in Higher Education. New Directions for Institutional Research.

Simmons, M. (1997). Leadership: The key to continual improvement. Total Quality Management, 8(2/), 273-274.

Sims, A. C., Bowles, J., \& Crosby, P. (1992). Does the Baldrige Award really work? Debate of several letters and discussion on the effectiveness of Malcolm Baldrige Award. Harvard Business Review, (January-Februar), 126-147.

Sims, S. J., \& Sims, R. R. (1995). Total Quality Management in Higher Education: Is It Working? Why Or Why Not? Wesport, CT: Praeger Greenwood.

Singh, C., \& Sareen, K. (2006). Effectiveness of ISO 9000 standards in Indian educational institutions: a survey. International Journal of Services Technology and Management, 7(4), 403-415.

Sorensen, C. W., Furst-Bowe, J. A., \& Moen, D. M. (2005). Quality and Performance Excellence in Higher Education: Baldrige on Campus. Bolton, MA: Anker.

Spanos, Y. E., Prastacos, G. P., \& Poulymenakou, A. (2002). The relationship between information and communication technologies adoption and management. Information \& Management, 39(8), 659-675.

Spathis, C., Petridou, E., \& Glaveli, N. (2002). An Empirical Study of Service Quality Perspectives in Public and Private Banks. In C. Zopounidis (Ed.), New Trends in Banking Management (pp. 3-19). Heidelberg: Springer Physica Verlag.

Sporn, B. (1999). Adaptive university structures: an analysis of adaptation to socioeconomic environments of US and European universities. London: Jessica Kingsley.

Stamoulas, A. (2006). Greece Before the Bologna Process: Confronting or Embracing Quality Assurance in Higher Education? Higher Education Policy, 19(4), 433-445.

Steger, W. P. (1999). Comparing news and editorial coverage of candidates of 1996 presidential nominating campaign. Presidential Studies Quarterly, 29(1), 40-72.

Stensaker, B. (2000). Quality as Discourse: An Analysis of Eexternal Audit Reports in Sweden 1995-1998. Tertiary Education and Management, 6(4), 305-317.

Stensaker, B. (2004). The transformation of organisational identities: interpretations of policies concerning the quality of teaching and learning in Norwegian higher education. Enschede: CHEPS, University of Twente, The Netherlands 
Strada, M. J. (2001). The humble syllabus as a creative catalyst. http://www.polsci.wvu.edu/facdis/NEAarticle.htm, Retrieved September 14,2008

Strang, D., \& Meyer, J. W. (1993). Institutional conditions for diffusion. Theory and Society, 22(4), 487-511.

Straubhaar, J., \& LaRose, R. (2002). Media Now: Communications Media in the Information Society (3rd ed). Belmont, CA: Wadsworth Publishing.

Sursock, A., \& Amaral, A. (2008). Self-Regulation in Europe: The Institutional Evaluation Programme. In A. Amaral, A. Rovio-Johansson, M. J. Rosa \& D. Westerheijden (Eds.), Essays in Supportive Peer Review (pp. 35-47). New York, NY: Nova Science Publishers.

Sursock, A., \& Toft Jensen, H., (2008). Self-Evaluation: How did universities take up their task? In A. Amaral, A. Rovio-Johansson, M. J. Rosa \& D. Westerheijden (Eds.), Essays in Supportive Peer Review (pp. 65-72). New York, NY: Nova Science Publishers.

Tashakkori, A., \& Creswell, J. W. (2007). Editorial: The New Era of Mixed Methods. Journal of Mixed Methods Research, 1(1), 3-7.

Tashakkori, A., \& Creswell, J. W. (2008). Envisioning the Future Stewards of the Social-Behavioral Research Enterprise. Journal of Mixed Methods Research, 2(4), 291-295.

Tashakkori, A., \& Teddlie, C. (1998). Mixed Methodology: Combining Qualitative and Quantitative Approaches. Thousand Oak, CA: Sage.

Tashakkori, A., \& Teddlie, C. (2003). Handbook of Mixed Methods in Social $\mathcal{E}$ Behavioral Research. Thousand Oaks: Sage.

Tashakkori, A., \& Teddlie, C. (2008). Quality in Inferences in Mixed methods Research: Calling for an Integrative Framework. In M. M. Bergman (Ed.), Advances in Mixed Methods Research. Theories and Applications (pp. 101-119). Los Angeles, CA: Sage.

Teddlie, C., \& Tashakkori, A. (2006). A general typology of research designs featuring mixed methods. Research in the Schools, 13(1), 12-28.

Teddlie, C., \& Tashakkori, A. (2009). Foundations of Mixed Methods Research: Integrating Quantitative and Qualitative Techniques in the Social and Behavioral Sciences. Thousand Oaks, CA: Sage.

Temple, P. (2005). The EFQM Excellence Model: Higher Education's Latest Management Fad? Higher Education Quarterly, 59(4), 261-274.

Theodorakioglou, Y. D., \& Tsiotras, G. D. (2000). The Need for the Introduction of Quality Management into Greek Healthcare. Total Quality Management Journal, 11(8), 1153-1165.

Thonhauser, T. (2005). Factors that relate to the successful implementation of ISO 9000 in Education: A comparison between the US and England. Pennsylvania State University, USA. 
Thonhauser, T., \& \& Passmore D.L. (2006). ISO 9000 in Education: a comparison between the United States and England. Research in Comparative and International Education, 1(2), 156-173.

Tolbert, P. S., \& Zucker, L. G. (1983). Institutional Sources of Change in the Formal Structure of Organizations: The Diffusion of Civil Service Reform, 1880-1935. Administrative Science Quarterly, 28(1), 22-39.

Triandis, H., Vassiliou, V., \& Massiakou, M. (1968). Three cross cultural studies of subjective culture. Journal of Personality and Social Psychology Monograph, 8(4), 2.

Trow, M. (1985). Comparative reflections on leadership in higher education. European Journal of Education, 20(2-3), 143-159.

Tsinidou, M., Gerogiannis, V., \& Fitsilis, P. (2010). Evaluation of the factors that determine quality in higher education: an empirical study. Quality Assurance in Education, 18(3), 227-244.

Tsiotras, G., \& Gotzamani, K. (1996). ISO 9000 as an entry key to TQM: the case of the Greek industry. International Journal of Quality $\mathcal{E}$ Reliability Management, 13(4), 64-76.

Tsoukalas, C., \& Panagiotopoulou, R. (1992). Education in Socialist Greece: Between Modernization and Democratozation. In T. Karyotis (Ed.), The Greek Socialist Experiment. New York, NY: Pella Publishing Company, INC.

Tuckman, G. (1978). Making News: A Study in the Construction of Reality New York: Fee Press.

Ursin, J. (2005). Whys and ways of internal quality assurance in Finnish universities. Paper presented at the Consortium of Higher Education Researchers (CHER). Jyväskylä.

Ursin, J. (2007). Universities as quality evaluators. Academic conceptions of quality and quality assurance [Yliopistot laadun arvioijina. Akateemisia ksityksi laadusta ja laadunvarmistuksesta] — Jyvaskyla, FN

Van Bruggen, Scheele, J. P., \& Westerheijden, D. F. (1998). To be continued... In J. P. Scheele, P. A. M. Maassen \& D. F. Westerheijden (Eds.), To be Continued...: Follow-Up of Quality Assurance in Higher Education. Utrecht: Elsevier/De Tijdstroom.

Van der Wende, M. C., \& Westerheijden, D. F. (2001). International Aspects of Quality Assurance with a Special Focus on European Higher Education. Quality in Higher Education, 7(3), 233-245.

Van Vught, F. A. (1989). Flexibility production and pattern management: two basic instruments of strategic planning for higher education institutions. Paper presented at the 29th Annual Forum of the Association for Institutional Research. 
Van Vught, F. A. (1996). The Humboltian University under Pressure: New forms for quality review in Western European higher education. In P. Maassen \& F. A. van Vucht (Eds.), Inside Academia (pp. 185-226). Utrecht: De Tijdstrom.

Van Vught, F. A., \& Westerheijden, D. F. (1993). Quality Management and Quality Assurance in European Higher Education: Methods and Mechanisms (Luxembourg, Commission of the European Communities, Education Training Youth, Studies No. 1). Education Training Youth: Studies.

Van Vught, F. A., \& Westerheijden, D. F. (1994). Towards a general model of quality assessment in higher education. Higher Education, 28(3), 355-371.

Van Vught, F. A., \& Westerheijden, D. F. (1996). Institutional evaluation and management for quality: the CRE programme: background, goals and procedures. CRE-Action, 107, 9-40.

Vazzana, G., Elfrink, J., \& Bachmann, D. P. (2000). A Longitudinal Study of Total Quality Management Processes in Business Colleges. Journal of Education for Business, 76(2 Nov-Dec), 69-74.

Veiga, J., Yanouras, J., Palmer, D., \& Boukis, S. (1987). Propensity to give up control in decision making groups: A comparison of U.S. and Greek managers. In managing in a global economy II. Paper presented at the Second International Conference of Eastern Academy of Management

Venieris, G., \& Cohen, S. (2004). Accounting Reform in Greek Universities: A slow moving process. Financial Accountability and Management, 20(2), 183-204.

Wang, J., Gibson, A. M., \& Slate, J. R. (2007). Effective teachers as viewed by students at a 2 year college: A multistage mixed analysis. Issues In Educational Research, 17(2), 272-295.

Watson, P. (2009). Regional themes and global means in supra-national higher education policy. Higher Education, 58(3), 419-438.

Weber, L. (2008). If you Believe you are Good, Try Institutional Evaluation! In A. Amaral \& Associates (Eds.), Essays on Supportive Peer Review (pp. 259-268), New York: Nova Science Publishers.

Weick, K. E. (1976). Educational Organizations as Loosely Coupled Systems. Administrative Science Quarterly, 21(1), 1-19.

Westerheijden, D. F. (1999). Where are the quantum jumps in quality assurance? Developments of a decade of research on a heavy particle. Higher education, 38(2), 233-254.

Westerheijden, D. F., Hulpiau, V., \& Waeytens, K. (2007). From Design and Implementation to Impact of Quality Assurance: An Overview of Some Studies into What Impacts Improvement. Tertiary Education and Management, 13(4), 1-18. 
Westerheijden, D. F., Beerkens, E., Cremonini, L., Huisman, J., Kehm, B., Kovac, A., et al. (2010). The first decade of working on the European Higher Education Area: The Bologna Process Independent Assessment - Volume 1 Detailed assessment report. s.l. [Brussels]: European Commission, DirectorateGeneral for Education and Culture.

Westphal, J. D., Gulati, R., \& Shortell, S. M. (1997). Customization or Conformity? An Institutional and Network Perspective on the Content and Consequences of TQM Adoption. Administrative Science Quarterly, 42(2), 366-394.

Williams, G. (1993). Total Quality Management in Higher Education: Panacea or Placedo? Higher Education, 25(3), 229-237.

Williams, J. R., \& Levy, P. E. (1992). The effects of perceived system knowledge on the agreement between self ratings and supervisor ratings Personnel Psychology, 45, 835-847.

Williams, W. J., \& Semlak, W. D. (1978). Structural effect of TV coverage on political agendas. Journal of Communication, 28, 114-119.

Winter, R. S. (1991). Overcoming Barriers to total quality management in colleges and universities. In L. A. Sherr \& G. G. Lozier (Eds.), Total Quality Management in Higher Education (pp. 53-62). San Francisco, CA: JosseyBass.

Witte, J. K. (2006). Change of degrees and degrees of change: comparing adaptations of European higher education systems in the context of the Bologna process. Enschede: CHEPS, University of Twente, The Netherlands.

Wysong, L. M. (2000). Leadership Behaviors and Effectiveness in Quality Improvements at Selected Institutions of Higher Education, University of Nebraska, Lincoln.

YPEPTH. (2003) Ministry of National Education and Religious Affairs. National Report Implementation of the Bologna Process. Athens, Greece.

YPEPTH. (2005). Ministry of National Education and Religious Affairs. National Report 2004- 2005. Towards the European Higher Education Area: Bologna Process. Athens, Greece.

YPEPTH. (2007). Ministry of National Education and Religious Affairs. National Report Hellas 2005-2007. Athens, Greece.

YPEPTH. (2008). Ministry of National Education and Religious Affairs. Bologna Process. Template for National Reports 2007-2009. Athens, Greece.

Zantanidis, S., \& Tsiotras, G. (1998). Quality Management: A New Challenge for the Greek Construction Industry. Total Quality Management International Journal, 9(7), 619-632.

Zucker, L. G. (1987). Institutional Theories of Organization. Annual Review of Sociology, 13(1), 443-464.

Zuckerman, A., \& Rhodes, L. (2000). Quality Assurance through ISO 9000. School Administrator, 57(6), 12-181. 